


\section{Propositions}

1. Human induced pluripotent stem-cell derived cardiomyocytes in combination with the multi-electrode array technique provide adequate input for predicting human cardiotoxicity without animal testing.

(this thesis)

2. Quantifying cardiotoxicity using in vitro models without physiologically based kinetic (PBK) modeling-facilitated reverse dosimetry underestimates the role of active metabolites. (this thesis)

3. Big data-based research will be the leading trend in life sciences.

4. PROTAC (Proteolysis-Targeting Chimera) technology will lead a revolution in small molecule drug development.

5. Doing a $\mathrm{PhD}$ requires a balance between self-doubting and selfconfidence.

6. Independent thinking becomes more important with the everincreasing use of social media platforms.

Propositions belonging to the thesis, entitled

A novel method for predicting human cardiotoxicity without animal testing

Miaoying Shi

Wageningen, 15 September 2021 



\section{A novel method for predicting human cardiotoxicity without animal testing}

Miaoying Shi 


\section{Thesis committee}

\section{Promotor}

Prof. Dr I.M.C.M. Rietjens

Professor of Toxicology

Wageningen University \& Research

\section{Co-promotor}

Dr H. Bouwmeester

Associate Professor of Toxicology

Wageningen University \& Research

Dr M. Strikwold

Associate Professor of Safety in the Food Chain

Van Hall Larenstein University of Applied Sciences, Leeuwarden

\section{Other members}

Prof. Dr R.F. Witkamp, Wageningen University \& Research

Dr A. Punt, Wageningen University \& Research

Dr R.H.S. Westerink, Utrecht University

Prof. Dr F.G.M. Russel, Radboud University Medical Center, Nijmegen

This research was conducted under the auspices of the Graduate School VLAG (Advanced studies in Food Technology, Agrobiotechnology, Nutrition and Health Sciences) 


\title{
A novel method for predicting human cardiotoxicity without animal testing
}

\author{
Miaoying Shi
}

Thesis

submitted in fulfilment of the requirements for the degree of doctor at Wageningen University

by the authority of the Rector Magnificus

Prof. Dr A.P.J. Mol, in the presence of the

Thesis Committee appointed by the Academic Board

to be defended in public

on Wednesday 15 September 2021

at 1.30 p.m. in the Aula. 
Miaoying Shi

A novel method for predicting human cardiotoxicity without animal testing 282 pages

$\mathrm{PhD}$ thesis, Wageningen University, Wageningen, the Netherlands (2021) With references, with summary in English

ISBN 978-94-6395-902-5

DOI https://doi.org/10.18174/550390 


\section{Table of content}

\section{Chapter 1}

General Introduction

Chapter 2

Evaluation of in vitro models of stem cell-derived cardiomyocytes to screen for potential cardiotoxicity of chemicals

\section{Chapter 3}

Integrating in vitro data and physiologically based kinetic modelling-facilitated reverse dosimetry to predict human cardiotoxicity of methadone

\section{Chapter 4}

In vitro-in silico-based prediction of inter-individual and interethnic variations in the dose-dependent cardiotoxicity of $R$ - and S-methadone in humans

\section{Chapter 5}

A new approach methodology for the prediction of (nor)ibogaineinduced cardiotoxicity in humans

\section{Chapter 6}

General Discussion

\section{Chapter 7}

Summary

\section{Annex}

Acknowledgments, About the author, List of publications, Overview of completed training activities 


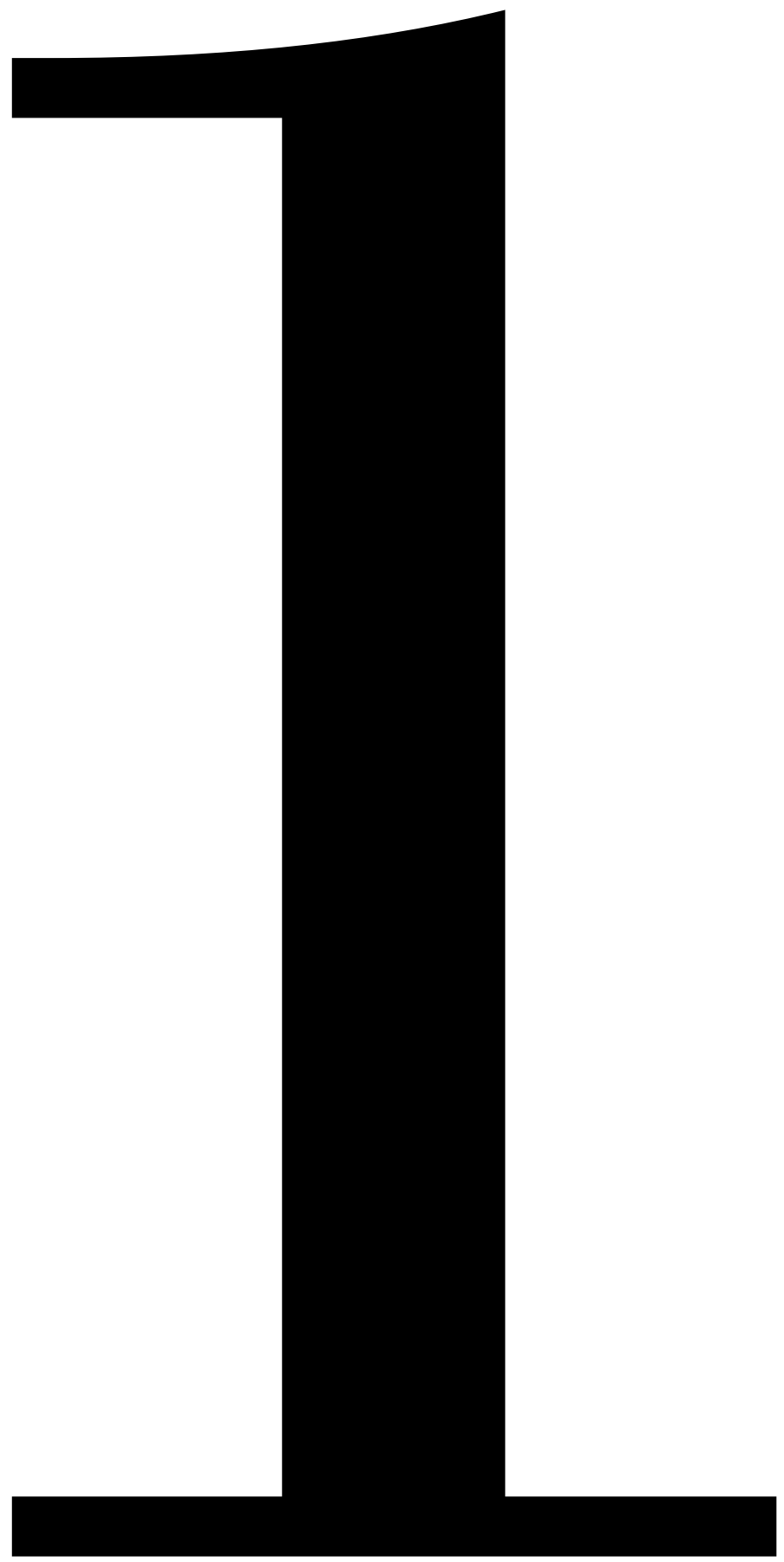




\section{Chapter 1}

General Introduction 


\section{Background information and aim of the present thesis}

Traditionally, risk assessment and safety evaluation for chemicals, including both nonpharmaceuticals and pharmaceuticals, has heavily relied on in vivo toxicity data obtained from animal studies which, however, have some inherent restrictions. One of the major limitations is the potentially poor correlation with the human effects due to inter-species differences in toxicodynamics and toxicokinetics (Bailey et al. 2014; Perel et al. 2007; Van Norman 2019). On the other hand, to better protect human health and the environment legislation and regulatory initiatives on the safety of industrial chemicals, food additives, cosmetics and pharmaceuticals have developed and point at the requirement on safety information of these compounds (European Commission (EC) 2007; EC 2008; EC 2009; EC 2010b; National Research Council (NRC) 2006). To fulfil the demand on toxicity testing for a large number of compounds, millions of animals are required to generate toxicity data, which is increasingly considered unacceptable from both ethical and economic perspectives (Rovida and Hartung 2009; Strikwold 2016). Due to these scientific and societal concerns, there is a significantly increased effort and interest in studying alternative approaches for animal testing. Already in the late 1950s Russel and Burch postulated the principle of the replacement, reduction and refinement of animal use, known as the 3Rs (Russell and Burch 1959). This had important implications in toxicological, biochemical and biological research for more than 60 years. In line with the 3Rs principles, the development of alternative testing strategies has become increasingly important, especially during the past decades, in toxicology testing for the risk assessment and safety evaluation of compounds (Andersen et al. 2019; European Medicines Agency (EMA) 2017; Taboureau et al. 2020). Over the past decades, many efforts from the academic and industrial setting as well as from regulatory authorities aimed at promoting the use of non-animal testing methods. The European Commission implemented the REACH Regulation for Registration, Evaluation, Authorization and restriction of CHemicals (2007/2006) (EC 2007), the Cosmetics Regulation (1223/2009) (EC 2009) and Directive (2010/63) (EC 2010a) which all include considerations to reduce the use of animals for scientific purposes and to enforce or strongly encourage the replacement of animal use. In the United States the Toxicology in the 21st Century program initiated by four government authorities proposed a paradigm shift in chemical risk assessment and testing strategies which encourages the use of human-related biological materials and the application of alternative animal methods enabling high-throughput toxicity screening for a diverse range of compounds and endpoints (Andersen et al. 2019; NRC 2007). The U.S. Environmental Protection Agency (EPA) officially announced a plan to 
eliminate all the requests for live mammal studies by 2035 (Grimm 2019). More recently, the term new approach methodology (NAM) is being proposed, which expands the concept of alternative methods for toxicity testing and includes more newly developed approaches such as organoids and omics approaches (ECHA 2016, ICCVAM 2018).

In light of the ongoing research on alternative methods or NAMs, a wide range of in vitro biological assays has been developed to screen a chemical of interest for different toxicity endpoints or to study the modes of action underlying the toxicity (Bernauer et al. 2005). The in vitro toxicity data generated by these assays provide useful information for hazard characterization. Clearly many in vitro experiments do not (yet) capture the information on the absorption, distribution and excretion (ADME) of chemicals (i.e. toxicokinetics), and thereby do not fully reflect the in vivo situation. To enable the use of in vitro assays in human risk assessment and safety evaluation of chemicals, a translation is required to convert the obtained in vitro concentration-response data to in vivo human dose-response data, taking into account toxicokinetics (Bell et al. 2018; Blaauboer 2010). Generally, toxicokinetics refers to the fate of chemicals and their metabolites within the body upon exposure. Toxicokinetic data on ADME of chemicals can be obtained using NAM including in vitro and in silico approaches (Punt et al. 2020). Collectively these ADME processes can be expressed using a set of mathematical equations in a method called physiologically based kinetic (PBK) modelling, quantitatively describing the time course of chemicals within the different compartments of a body upon exposure to a certain dose. PBK models link the external exposure doses of a chemical to the internal concentrations of that chemical in the systemic circulation or target organs (Lin and Fisher 2020). The PBK modelling approach could be especially powerful for the risk assessment and safety evaluation when integrated with biological data obtained from in vitro assays for the critical toxicological endpoint of interest. One good example of such an integration is so-called PBK modelling-based reverse dosimetry in which the in vitro effective concentrations are set equal to the internal concentrations in the blood or target organs and are subsequently converted to the corresponding external doses. By repeating the same procedure for each effective concentration obtained in the in vitro toxicity assay the concentrationresponse curves can be translated to predicted in vivo dose-responses curves from which points of departure (PoDs) can be derived to define the safe exposure level of a chemical (Louisse et al. 2010; Rietjens et al. 2011). To date this quantitative in vitro in vivo extrapolation (QIVIVE) has shown to adequately predict the in vivo toxicity of chemicals for various toxicity endpoints including developmental toxicity (Louisse et al. 2010; Strikwold et al. 2013, 2017), 
nephrotoxicity (Abdullah et al. 2016), liver toxicity (Ning et al. 2019, Gilbert-Sandoval et al., 2020) and neurotoxicity (Algharably et al. 2021; Omwenga et al. 2021; Zhao et al. 2019).

To further promote the use of this new approach methodology for human risk assessment and safety evaluation it is essential to explore its potential applicability for a broader range of toxicity endpoints and for the human situation. The human heart has been shown to be the target organ when exposed to a wide range of chemicals including heavy metals, natural alkaloids, pesticides (organophosphate) and pharmaceuticals, and cardiotoxicity has been considered as one of the most important toxicity endpoints in the safety testing of chemicals (Kratz et al. 2017; Krishna et al. 2020). Especially for drug development cardiotoxicity is reported to be the major cause of drug failure and withdrawal, accounting for $27 \%$ of drug failure during the early stage of drug development, and 16\% of drug withdrawals from the market in Europe and the United States (Ovics et al. 2020; Pang et al. 2019; Siramshetty et al. 2016). Additionally, because of the severe health consequences of pro-arrhythmic drugs, in vivo electrophysiological cardiotoxicity studies using whole animals are compulsory for all drug candidates (The International Council for Harmonization of Technical Requirements for Pharmaceuticals for Human Use (ICH) 2005). Clearly this has resulted in a need to use a large number of experimental animals to exclude this unintended side effect. A recent report from the European Union Reference Laboratory for alternatives to animal testing (EURL ECVAM) revealed that around 382,000 animals were used for cardiovascular related research in Europe in 2017 (Zuang et al. 2021). Furthermore, proofs-of-principle for in vivo dose-response curves for toxicity predicted by PBK modeling-based reverse dosimetry obtained so far often relate to predicted toxicity in experimental animals, because for experimental animals predicted data could be evaluated based on comparison to existing animal toxicity data. This leaves the need to obtain proofs-of-principle for application and evaluation of the PBK modeling-based reverse dosimetry approach to predict human toxicity.

\subsection{Aim of the present thesis}

The present thesis aims to provide proofs-of-principle for using PBK modeling-based reverse dosimetry of in vitro data for the prediction of cardiotoxicity in humans, thereby providing a novel testing strategy for cardiac safety studies. Methadone and ibogaine, two anti-addiction drugs with known in vivo cardiotoxicity, were selected as model compounds. The developed QIVIVE approach could contribute to alternatives of animal testing/ non-animal based NAMs for risk assessment and safety evaluation of chemicals. 


\section{Chemical-induced cardiotoxicity}

Potential cardiotoxicity including electrical and contractile dysfunction of cardiomyocytes can be attributed to different mechanisms (Ovics et al. 2020). Chemicals can interfere with the ion channels and receptors involved in the maintenance of membrane potentials, resulting a change in cardiac electrophysiology (Priest and McDermott 2015). Furthermore, chemicals such as doxorubicin can influence both the electrical and contraction function via disrupting the intracellular $\mathrm{Ca}^{2+}$ signaling (Burridge et al. 2016; Hanna et al. 2014), while chemicals such as several anti-cancer reagents are reported to induce aberrant contraction due to their cytotoxic effects on cardiomyocytes (Doherty et al. 2013; Zhao and Zhang 2017). Particularly, the electrophysiological alterations including ventricular arrhythmias are the most noticeable chemical-induced forms of cardiotoxicity (Ovics et al. 2020) and thus electrophysiological cardiotoxicity was chosen as the toxicity endpoint in the present thesis.

Normal cardiac contractions are triggered by the electrical signals called action potentials, which are generated by the changes of inward and outward ion fluxes across the cell membrane. More than 60 ion channels, ion pumps, ion exchangers and membrane receptors are involved in the initiation of action potentials in human cardiomyocytes (Hondeghem and De Clerck 2012; Tripathi et al. 2011). Figure 1 shows the major ion channels and ion fluxes involved in the initiation of action potentials in human ventricular cardiomyocytes. The action potential starts with an upstroke induced by inward sodium currents $\left(\mathrm{I}_{\mathrm{Na}}\right)$ through voltage-gated sodium $\left(\mathrm{Na}^{+}\right)$ channels (phase 0 ). Then a rapid repolarization occurs (phase 1) driven by transient outward potassium $\left(\mathrm{K}^{+}\right)$currents $\left(\mathrm{I}_{\mathrm{to}}\right)$, which is followed by a plateau phase (phase 2) resulting from the balance between potassium efflux mainly mediated by rapid and slow delayed rectifier $\mathrm{K}^{+}$ channels (corresponding to currents $\mathrm{I}_{\mathrm{Kr}}$ and $\mathrm{I}_{\mathrm{Ks}}$, respectively) and calcium $\left(\mathrm{Ca}^{2+}\right)$ influx through $\mathrm{Ca}^{2+}$ channels. The plateau ends with the inactivation of the $\mathrm{Ca}^{2+}$ channels, while delayed rectifier $\mathrm{K}^{+}$channels remain open leading to repolarization (phase 3). At the end of phase 3, intracellular $\mathrm{Na}^{+}$and $\mathrm{Ca}^{2+}$ are transported out of cells by $\mathrm{Na}^{+} / \mathrm{K}^{+}$ATPase and $\mathrm{Na}^{+} / \mathrm{Ca}^{2+}$ exchangers. The membrane potential finally returns to the resting potential (phase 4) mainly driven by $\mathrm{K}^{+}$efflux through the inward rectifier $\mathrm{K}^{+}$channel (corresponding to current $\mathrm{I}_{\mathrm{k} 1}$ ) (Huang 2017; Jeevaratnam et al. 2018; Rougier and Abriel 2016). The sum of depolarization and repolarization is defined as the action potential duration (APD). The phases of the action potential can be detected by using electrodes placed on the skin of a person and is reflected by the electrocardiogram (ECG). As illustrated in Figure 1, the QRS complex is generated in phase 0 and corresponds to ventricular depolarization. The ST interval and T wave represent the 
plateau and repolarization phase of the action potential, respectively. The QT interval is defined as the duration from the beginning of the QRS complex to the end of the T wave, reflecting the ventricular depolarization and repolarization (Fermini and Fossa 2003; Hondeghem and De Clerck 2012). Additionally, the QT interval is usually corrected for heart rate (QTc) in order to minimize the influence of heart rate variability and allow a better comparison of individual values with refence values in the clinic (Postema and Wilde 2014). Bazett's correction $\left(\mathrm{QTc}=\mathrm{QT} / \mathrm{RR}{ }^{1 / 2}\right.$, where $\mathrm{RR}$ is defined as the interval between two QRS complexes and expressed in milliseconds) and Fridericia's correction (QTc=QT/ RR ${ }^{1 / 3}$ ) are two of the most commonly used formulae for the correction of the QT interval for heart rate (Postema and Wilde 2014).

Chemical-induced cardiac adverse effects are often caused by undesired interactions with above-mentioned ion channels or receptors involved in the regulation of action potentials (Hondeghem and De Clerck 2012). A decrease in repolarization (outward) currents and/or an increase in depolarization (inward) currents will prolong the ventricular APD (Fermini and Fossa 2003; Ovics et al. 2020; Roden 2008). In the human ECG, a delayed ventricular APD is reflected by a prolonged QT interval, which is associated with increased incidences of torsade de pointes (TdP), a life-threatening polymorphic ventricular tachyarrhythmia (rapid heart rhythms) with rapid and twisting QRS complexes around the isoelectric baseline (Ewart et al. 2012; Fermini and Fossa 2003; Kannankeril et al. 2010). Such QT prolongation and arrhythmia can also be caused by drugs, which has been the main reason for the discontinuation and withdrawal of several drugs (Hondeghem and De Clerck 2012). It is generally considered that the rapid delayed rectifier $\mathrm{K}^{+}$channels encoded by the human ether-à-go-go-related gene (hERG) play an important role in repolarization, as the blockage of the hERG channel is associated with prolonged APD and QT intervals (Martin et al. 2004; Sanguinetti et al. 1995; Thomas et al. 2006). Therefore, regulators require to evaluate the potential for delayed repolarization for all drug candidates using an in vitro hERG channel inhibition assay in the preclinical stage (ICH 2005). 


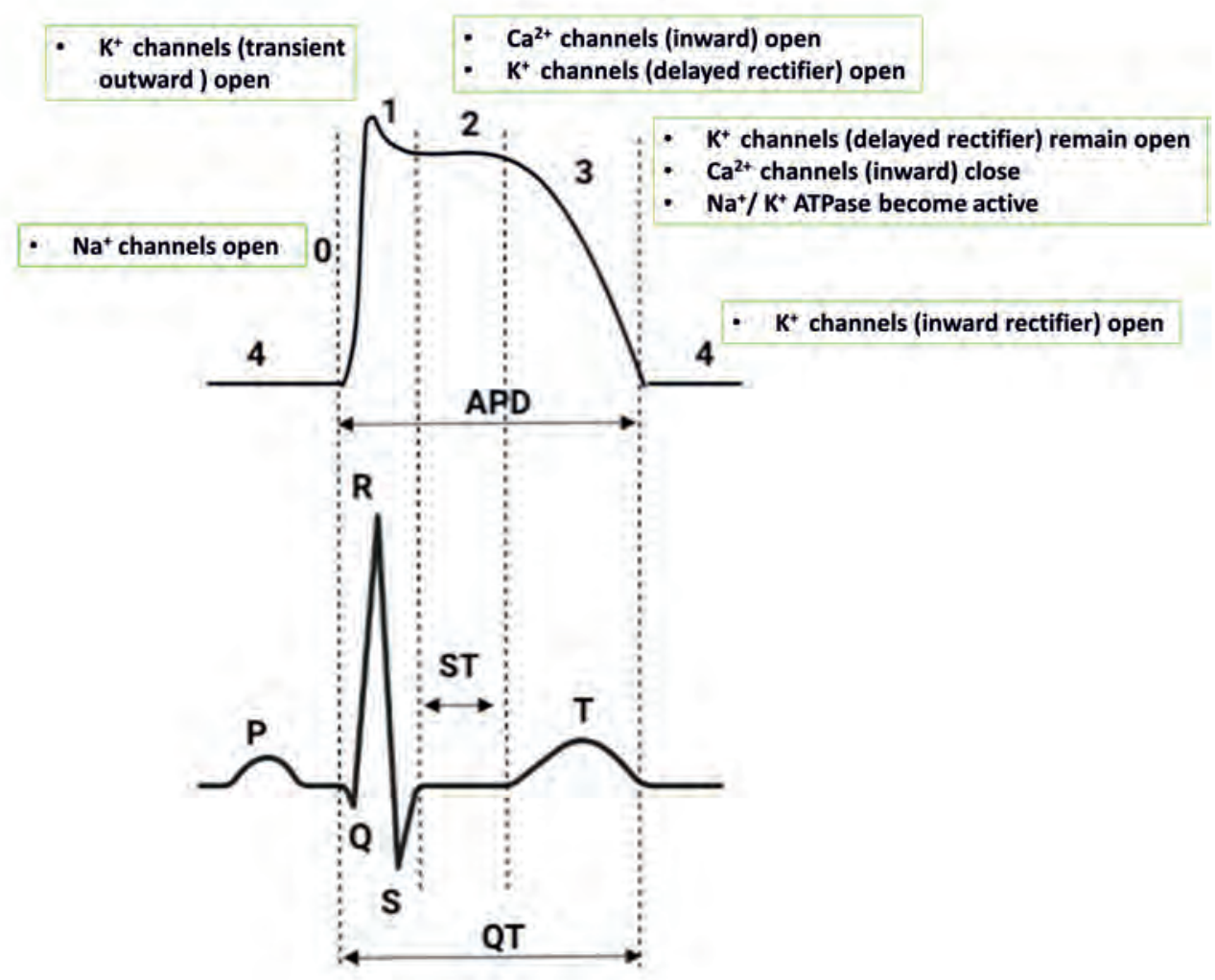

Figure 1 Comparison between action potentials in human ventricular cardiomyocytes and the human electrocardiogram (ECG). The upper part of the figure shows the major ion channels and fluxes involved in the initiation of action potentials in human ventricular cardiomyocytes. Phase 0 : the rapid depolarization is induced by inward sodium current $\left(\mathrm{I}_{\mathrm{Na}}\right)$ through the voltage-gated sodium $\left(\mathrm{Na}^{+}\right)$ channels. Phase 1: the rapid repolarization caused by the activation of the transient outward potassium currents $\left(\mathrm{I}_{\mathrm{to}}\right)$. Phase 2: the plateau phase is the result of the balance between potassium $\left(\mathrm{K}^{+}\right)$efflux mainly mediated by rapid and slow delayed rectifier $\mathrm{K}^{+}$channels and calcium $\left(\mathrm{Ca}^{2+}\right)$ influx through $\mathrm{Ca}^{2+}$ channels. Phase 3: the repolarization is caused by the inactivation of the $\mathrm{Ca}^{2+}$ channels and the ongoing $\mathrm{K}^{+}$efflux via delayed rectifier $\mathrm{K}^{+}$channels. At the end of phase 3 , intracellular $\mathrm{Na}^{+}$and $\mathrm{Ca}^{2+}$ are transported out of cells by $\mathrm{Na}^{+} / \mathrm{K}^{+}$ATPase and $\mathrm{Na}^{+} / \mathrm{Ca}^{2+}$ exchanger. Phase 4: the membrane potential returns to the resting potential mainly driven by $\mathrm{K}^{+}$efflux through the inward rectifier $\mathrm{K}^{+}$channel (Rougier and Abriel, 2016; Huang, 2016; Jeevaratnam et al., 2018). The bottom part of the figure shows the human ECG where the QRS complex, ST interval, and T wave represent the depolarization, the plateau and the repolarization phase of ventricular action potentials, respectively (Fermini and Fossa 2003; Hondeghem and De Clerck 2012). APD, action potential duration. 


\section{Model compounds}

To provide proofs-of-principle for using PBK modeling-based reverse dosimetry of in vitro data for the prediction of cardiotoxicity in humans, two anti-addiction drugs were used in the present thesis as model compounds. The model compounds were chosen based on the following criteria 1) available evidence showing that exposure to the compound is associated with QT prolongation or arrythmia in human and 2) the presence of both in vivo human kinetic and QT data on the compound, which enable the evaluation of the developed PBK models and of the predicted dose-response curves for cardiotoxicity against observed data. The model compounds thus selected were methadone an ibogaine.

\subsection{Methadone}

\subsubsection{Cardiotoxicity of methadone}

Methadone (Figure 2) is a synthetic opioid agonist prescribed for pain relieve and used as a substitute to reduce the withdrawal syndrome induced by other opiates (Alinejad et al. 2015; Behzadi et al. 2018). As a high-efficacy and low-priced anti-addiction drug, methadone is extensively used in the clinic, which however has been reported to induce QTc prolongation and TdP in patients receiving opioid maintenance treatment (Fareed et al. 2013; Justo et al. 2006; Wedam et al. 2007). Several in vitro studies employing electrophysiological-based techniques or heterologously transfected cell models revealed that methadone-induced QTc prolongation can be explained by the inhibitory effects of methadone on hERG channels (Eap et al. 2007; Kuryshev et al. 2010). Methadone is administered as the racemate with a 1:1 mixture of the R- and S-enantiomer (Eap et al. 2002). The two enantiomers have different potencies for both pharmacological effects and cardiotoxicity with mainly S-methadone being responsible for the cardiac adverse effects (Ansermot et al. 2010; Eap et al. 2007).

\subsubsection{ADME of methadone}

Methadone is a lipophilic basic drug that can be rapidly absorbed following oral administration with detectable blood concentrations occurring within 15 to $45 \mathrm{~min}$ (Eap et al. 2002; Inturrisi et al. 1987; Wolff et al. 1997). After the oral dosing the time to reach peak plasma concentrations is $2.5 \mathrm{~h}$ on average and varies from 1 to $5 \mathrm{~h}$, being not dependent on the dose (Lugo et al. 2005; Wolff et al. 1997). The oral bioavailability of methadone is generally high with an average value of $82 \%$ but shows large variability among individuals (ranging from 41 to $99 \%$ ) (Dale et al. 2004; Kharasch et al. 2009). Following absorption in the gastrointestinal 
tract, methadone is well distributed throughout the body as reflected by a volume of distribution amounting to 4 1/kg (de Vos et al. 1995; Lugo et al. 2005). As methadone is a highly lipophilic compound, it preferably distributes and accumulates in tissues such as brain, gut, kidney, liver and lung (Barbosa Neto et al. 2015). Methadone is extensively bound to plasma protein with a mean unbound fraction of 0.14 , which shows variation (i.e. ranging between 0.034 to 0.22 ) (Eap et al. 1990; Olsen 1973; Romach et al. 1981; Wilkins et al. 1997). As a basic drug methadone is primarily bound to alpha1-acid glycoprotein (AAG) and the level of AAG could increase in certain pathologic conditions such as cancer and opioid addiction (Abramson 1982; Lugo et al. 2005; Romach et al. 1981). This variability may partly explain the variation in the methadone plasma binding.

The metabolism of methadone mainly occurs in the liver where methadone is $\mathrm{N}$ demethylated followed by spontaneous cyclisation resulting in formation of its primary metabolite 2-ethylidene-1,5-dimethyl-3,3-diphenylpyrrolidine (EDDP) which is subsequently metabolized to a secondary metabolite, 2-ethyl-5-methyl-3,3-diphenylpyrroline (EMDP) via Ndemethylation (Moody et al. 1997; Verebely et al. 1975) (Figure 2). In vitro and human studies revealed that the major enzymes mediating the conversion of methadone to EDDP are cytochromes P450 (CYP)2B6, CYP3A4 and to a lesser extent CYP2C19. CYP2C19 and CYP2B6 are reported to be stereoselective towards the conversion of R-and S-methadone, while CYP3A4 appeared to be non stereoselective (Eap et al. 2007; Foster et al. 1999; Gerber et al. 2004; Totah et al. 2007). It is important to note that an up to 17-fold variation in methadone blood concentrations has been observed in subjects exposed to a certain dose of methadone, which could partly be attributed to the inter-individual variability in the CYP enzymes involved in its metabolism (Eap et al. 2002; Li et al. 2008). After oral dosing, methadone and its metabolites can be excreted via both urine and feces with a percentage of the oral dose up to $57 \%$ and $45 \%$, respectively (Barbosa Neto et al. 2015; Dean 2004). 


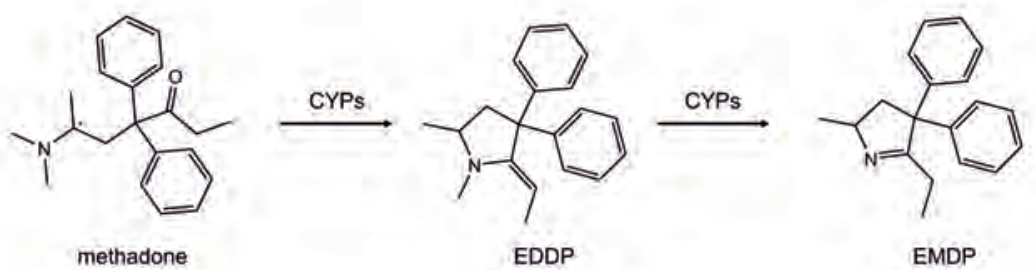

Figure 2 Metabolic conversion of methadone to 2-ethylidene1,5-dimethyl-3,3-diphenylpyrrolidine (EDDP), and 2-ethyl 5-methyl-3,3-diphenylpyrroline (EMDP) by cytochromes P450 (CYPs). The asterisk indicates the chiral centre.

\subsection{Ibogaine}

\subsubsection{Cardiotoxicity of ibogaine (and noribogaine)}

Ibogaine (Figure 3) is a natural alkaloid derived from the root bark of the West African shrub Tabernanthe iboga. Originally it was used as a spiritual ceremony agent by West African tribes given its potential psychoactive and hallucinogenic effects (Davis et al. 2017; Mash et al. 2018). In modern medicine ibogaine has been used as an anti-addictive drug in New Zealand while it is banned for human use and only used for research aims in most countries due to its psychoactive properties (Mash et al. 2018; Noller et al. 2018). To date, many in vitro and in vivo studies revealed that both ibogaine and its major metabolite noribogaine (Figure 3) show neurobiological effects in human and animals with the potential to reduce opioid dependence and depressive symptoms (Baumann et al. 2001a; Baumann et al. 2001b; Mash et al. 2016; Mash et al. 2001; Noller et al. 2018). Despite the promising pharmacological efficacy observed in preclinical studies, the potential cardiotoxicity hampers their legal uses in the clinic (Schep et al. 2016). Over decades, results from several human case studies indicated that ibogaine could be associated with QTc prolongation, TdP and several fatalities in subjects following oral administration of high doses of ibogaine (Asua 2013; Grogan et al. 2019; Hildyard et al. 2016; Hoelen et al. 2009; O'Connell et al. 2015; Paling et al. 2012; Steinberg and Deyell 2018; Vlaanderen et al. 2014). More recently, a phase 2 clinical trial revealed that noribogaine induced a dose-dependent QTc prolongation in opioid-dependent patients at dose levels of 60, 120 and $180 \mathrm{mg}$ noribogaine (Glue et al. 2016). The underlying mechanism of ibogaine and noribogaine-induced cardiotoxicity is not fully clear but could be related to their inhibitory effect on hERG channels observed in in vitro studies. In these studies, ibogaine and noribogaine 
were equally potent in blocking hERG channels as measured by using the patch clamp technique (Alper et al. 2016; Koenig et al. 2014; Rubi et al. 2017).

\subsubsection{Combined effects of ibogaine and noribogaine}

Considering that ibogaine and noribogaine show the potential to inhibit hERG channels, both compounds could induce cardiotoxic effects at the internal site of interest (i.e. heart) upon oral exposure to ibogaine. Assuming that the cardiotoxic effects of ibogaine and noribogaine are additive, the combined effective concentration of ibogaine and noribogaine could be described as the ibogaine equivalent concentration by applying the dose addition method used for the hazard assessment of a mixture of chemicals showing similar toxicity by a similar mode of action. Thus the so-called toxic equivalency (TEQ) approach can be applied, which assesses the combined effect of chemical mixtures taking the potency of each mixture component into account by defining the toxic equivalency factor (TEF). The TEF reflects the ratio of the toxicity of an individual chemical relative to the toxicity of the index chemical and the total toxic concentration can be calculated as TEQ which is the sum of the concentration of the mixture components multiplied by their respective TEFs (Bil et al. 2021; European Food Safety Authority (EFSA) 2013; EFSA 2019; EPA 2000; EPA 2010).

\subsubsection{ADME of ibogaine (and noribogaine)}

Due to the low number of studies on ibogaine and noribogaine, only limited information on human pharmacokinetics is available in the literature. Ibogaine is a lipophilic and basic compound. Upon oral administration of ibogaine, the peak plasma concentration occurred at 1 $\mathrm{h}$ and $1.7 \mathrm{~h}$ with a given dose of 20 and $700 \mathrm{mg}$, respectively (Glue et al. 2015b; Mash et al. 2001). While for noribogaine the time to reach peak plasma concentrations was longer, amounting to 3 to $4 \mathrm{~h}$ with the given doses ranging from 3 to $180 \mathrm{mg}$ without substantial differences between healthy subjects (Glue et al. 2015a) and opioid dependent individuals (Glue et al. 2016). Ibogaine and noribogaine are reported to distribute in the human body with particularly high concentrations found in liver, spleen, lung and brain (Kontrimavičiūtè et al. 2006; Litjens and Brunt 2016). Additionally, clinical studies observed a high volume of distribution of noribogaine (18-39 1/kg), reflecting substantial distribution of noribogaine to body tissues as well as its high lipophilicity (Glue et al. 2015a; Glue et al. 2016). In the liver ibogaine is rapidly and extensively metabolized to its primary metabolite noribogaine via Odemethylation (Obach et al. 1998; Glue et al., 2015; Litjens and Brunt, 2016). An in vitro microsomal incubation study identified that CYP2D6 was the major enzyme involved in the metabolism of ibogaine and CYP2C9 and CYP3A4 appeared to have minor contributions 
(Obach et al., 1998). Glue et al. (2015b) found that the plasma concentration of ibogaine in subjects treated with a CYP2D6 inhibitor was 27-fold higher than that in placebo-treated subjects, confirming a major role of CYP2D6 in the conversion of ibogaine to noribogaine. Subsequently, noribogaine is converted to noribogaine glucuronide (Glue et al., 2016; Glue et al., 2015b) (Figure 3). Glue et al. (2015a) reported that only small amounts of noribogaine glucuronide were formed after an oral dose of noribogaine, suggesting a minor contribution of glucuronidation to the total clearance of noribogaine. Ibogaine can be rapidly eliminated from the human body with a half-life of $2.5 \mathrm{~h}$ at a given dose of $20 \mathrm{mg}$ (Glue et al., 2015b) while noribogaine is slowly eliminated with a half-life of $30 \mathrm{~h}$ after an oral dose of $120 \mathrm{mg}$ noribogaine (Glue et al., 2016). It is not clear whether ibogaine could be excreted via urine while the urinary excretion of noribogaine and its glucuronide is low, accounting for $1.4-3.9 \%$ of the dose administered after a single oral dose of noribogaine (Glue et al. 2015a). Both ibogaine and noribogaine are found in human bile and excreted via the gastrointestinal tract (Alper 2001; Kontrimavičiūtė et al. 2006). Noribogaine was suspected to undergo enterohepatic circulation in certain individuals upon oral administration of noribogaine (Glue et al. 2015a).

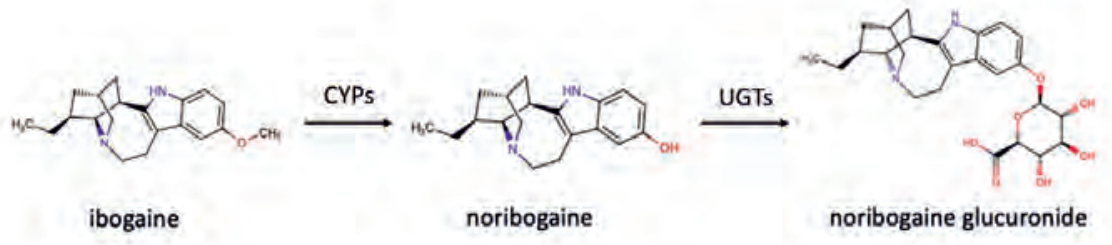

Figure 3 Metabolic pathway of ibogaine to noribogaine by cytochromes P450 (CYPs) and subsequent conversion of noribogaine to noribogaine glucuronide by glucuronosyltransferases (UGTs).

\section{Approaches used in the present thesis}

\subsection{In vitro model for cardiotoxicity}

Up to date, several in vitro cell- and tissue-based models are being developed to identify the electrophysiological cardiotoxicity induced by chemicals. Generally, cell-based assays for electrophysiological cardiotoxicity employ non-heart cell lines transfected with one or more ion channel(s) or cardiomyocytes derived from embryonic stem cells or induced pluripotent stem cells, or primary cardiomyocytes (Burnett et al. 2021). When combined with the use of the patch clamp technique, ion channel cell models can provide information on the interaction between chemicals and ion channels and thus on the mechanism underlying cardiotoxicity 
(Dunlop et al. 2008; Hamill et al. 1981). Functional cardiomyocytes can be integrated with a broad range of techniques such as patch clamping, multi-electrode array (MEA), intracellular calcium imaging voltage sensitive dyes and impedance measurement, which allows a comprehensive assessment of chemical-induced cardiotoxicity based on different electrophysiological endpoints ( $\mathrm{Li}$ et al. 2016; Walker et al. 2017). Recently, the heart slices biomimetic culture system, a tissue-based model, has been demonstrated to be a promising platform for the detection of cardiotoxicity in a model close to the in vivo physiological situation (Miller et al. 2020; Ou et al. 2019). Additionally, with the development of threedimensional (3D) cell culture techniques, microphysiological heart models and heart-on-chip systems have been applied as proof-of-principle tools for the detection of cardiotoxicity (Burnett et al. 2021; Zuppinger 2019). Table 1 summarizes the advantages and disadvantages of typical in vitro cardiotoxicity testing models. Among these, models employing stem cellderived cardiomyocytes are frequently used for cardiotoxicity screening of chemicals, given their ability to detect the cardiotoxic effects resulting from multiple channels and being robust and easy to use. Therefore, the present thesis focused on two stem cell-based in vitro models using mouse embryonic stem cell-derived cardiomyocytes (mESC-CMs) and human induced pluripotent stem cell-derived cardiomyocytes (hiPSC-CMs) evaluating their applicability domain for cardiotoxicity testing. 
Table 1 Summary of advantages and limitations of in vitro testing assays for electrophysiological cardiotoxicity

\begin{tabular}{|c|c|c|}
\hline In vitro testing assay & Advantages & Limitations \\
\hline Ion channel assay & $\begin{array}{l}\text { Mechanistic information on the } \\
\text { effects of chemical on ion } \\
\text { channels and their role in } \\
\text { cardiotoxicity }\end{array}$ & 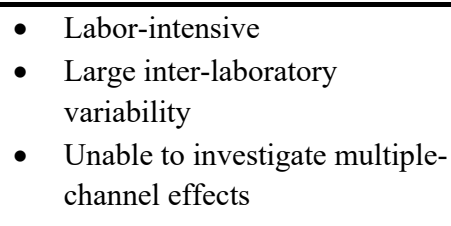 \\
\hline Patch clamping & $\begin{array}{l}\text { - Highly sensitive readouts } \\
\text { - Multiple electrophysiological } \\
\text { endpoints }\end{array}$ & $\begin{array}{l}\text { - } \text { Invasive to cells } \\
\text { - } \text { Low throughput } \\
\text { - }\end{array}$ \\
\hline $\begin{array}{l}\text { Voltage sensitive } \\
\text { dyes }\end{array}$ & $\begin{array}{ll}\text { - } & \text { High throughput } \\
\text { - } & \text { Highly sensitive readouts }\end{array}$ & - Cytotoxic effect of dyes \\
\hline Multi-electrode array & $\begin{array}{l}\text { Less inter-laboratory variability } \\
\text { compared to ion channel assay } \\
\text { - Enables the investigation on } \\
\text { multiple-channel effects } \\
\text { - Acute and chronic exposure }\end{array}$ & $\begin{array}{ll}\text { - } & \text { High costs } \\
\text { - } & \text { Medium throughput }\end{array}$ \\
\hline 3D heart models & $\begin{array}{l}\text { Mimic the structure and function } \\
\text { of native cardiac tissue }\end{array}$ & 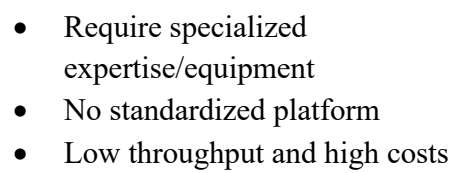 \\
\hline
\end{tabular}

\subsubsection{Mouse embryonic stem cell-derived cardiomyocyte (mESC-CM) beating arrest assay}

Mouse embryonic stem cells were first derived from developing mouse blastocysts and can spontaneously differentiate into beating cardiomyocytes which express the major cardiac contractile proteins, ion channels and receptors (Abassi et al. 2012; Evans and Kaufman 1981; Himmel 2013; Maltsev et al. 1994; Wobus et al. 1991). Nicolas et al. (2015) successfully detected the inhibitory effects of various ion channel (receptor) blockers on the beating of mESC-CMs, suggesting the potential of mESC-CMs for screening the cardiotoxicity of chemicals. In the present thesis the mESC-CM beating arrest assay was set up based on the method developed by Nicolas et al. (2015). As illustrated in Figure 4, mouse embryonic stem D3 cells were first aggregated as small cell droplets on the lid of a 96-well plate which allows the formation of embryoid bodies (EBs). After two-day incubation EBs were transferred to a 
petri-dish for an additional two-day culture and subsequently transferred to 48-well plates where cells differentiated into beating cardiomyocytes from day 10 onwards. Upon compound exposure, the number of beating cells were counted microscopically and compared to the number of beating cells in the corresponding medium controls. The cardiotoxicity of a chemical is reflected by its inhibitory effect on beating cardiomyocytes. The details of culturing, exposure and analysis of the mESC-CM are shown in Chapter 2.

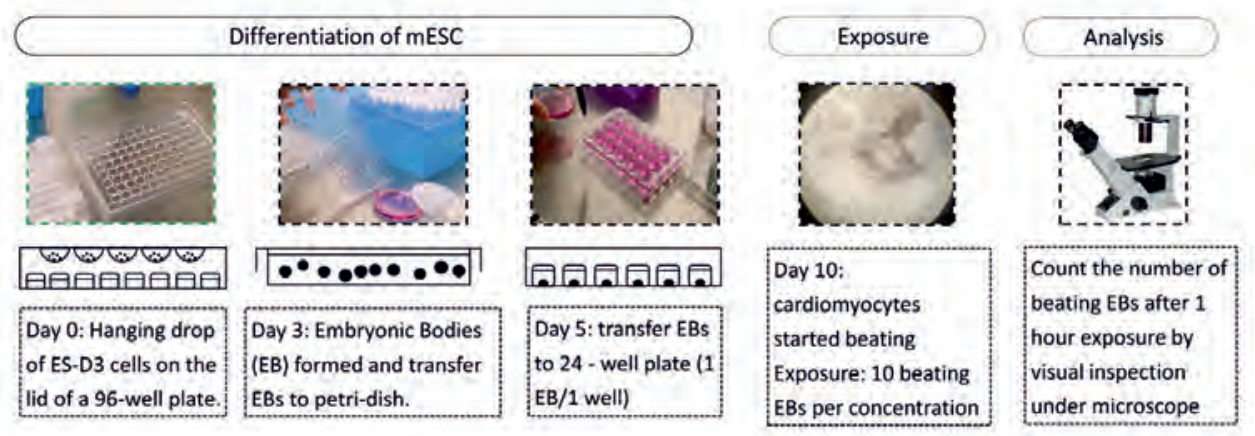

Figure 4 The scheme of the mouse embryonic stem cell derived cardiomyocyte (mESC-CM) beating arrest assay.

\subsubsection{Human induced pluripotent stem cell-derived cardiomyocyte (hiPSC-CM) multi- electrode array (MEA) assay}

In 2009 hiPSCs were successfully differentiated to functional cardiomyocytes for the first time (Zhang et al. 2009). Since then, hiPSC-CMs combined with different techniques have been intensively used for cardiotoxicity screening, drug development and heart disease modeling (Chang and Mummery 2018; Li et al. 2020; Walker et al. 2017). Many studies demonstrated that hiPSC-CMs express the major cardiac ion pumps and exchangers and membrane receptors known to be present in human cardiomyocytes (Garg et al. 2018; Karakikes et al. 2015; Ma et al. 2011). In recent years hiPSC-CMs combined with the MEA technique have been frequently used for cardiac safety assessments providing comprehensive information on chemical-induced multiple electrophysiological effects (Harris et al. 2013; Kitaguchi et al. 2017; Nozaki et al. 2017; Zwartsen et al. 2019). Figure 5 shows how the hiPSC-CM MEA assay was performed in the present thesis. Commercially available hiPSC-CMs were seeded on electrodes on a six-well MEA chip where a monolayer of beating cells was obtained at 7 to 8 days post seeding. Subsequently the electrical activity of beating cells was measured by the MEA system, reflected by the real-time waveform of extracellular field potential (middle picture in Figure 5). Upon 
chemical exposure, the changes of the extracellular field potential were recorded and analyzed using specific software. The chemical-induced cardiotoxic effects could be characterized using multiple electrophysiological parameters such as sodium spike amplitude, field potential duration (FPD) and RR-interval (duration between two depolarization peaks). These parameters are considered to specifically correspond to the parameters observed in a human ECG (Zwartsen et al. 2019) and thus can be used as in vitro surrogate indicators for the in vivo cardiotoxicity. The details of culturing, exposure, interpretation of the electrophysiological parameters and data analysis are described in Chapter 2, 3 and 5.
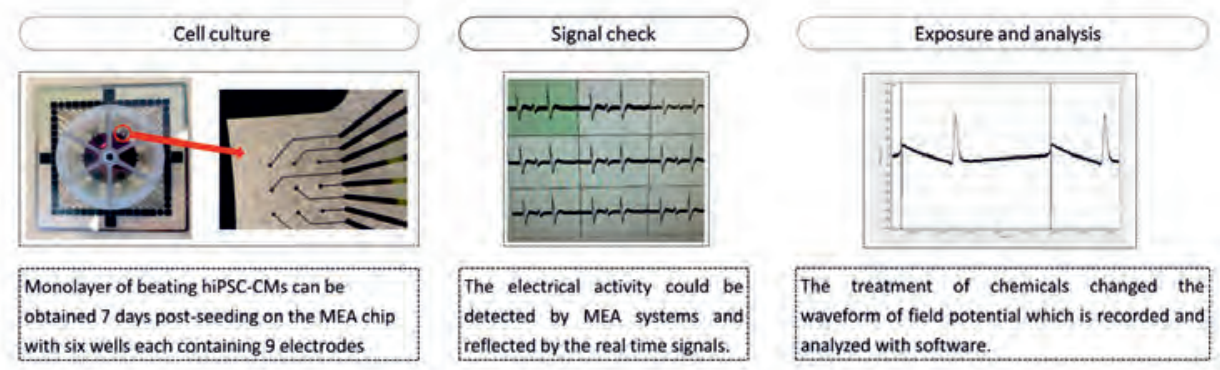

Figure 5 The scheme of the human induced pluripotent stem cell-derived cardiomyocyte (hiPSCCM) multi-electrode array (MEA) assay.

\subsection{Physiologically based kinetic (PBK) modelling}

PBK modelling is a mathematical in silico approach that quantitatively describes the ADME process of chemicals and their metabolites within the body (Chiu et al. 2007; Rietjens et al. 2011). In the PBK model, body tissues and organs are described as different compartments each defined by a set of mathematical equations, which can describe the time dependent ADME for chemicals and their metabolites in the body. PBK models can predict the internal concentrations (in plasma/blood or a target organ) upon exposure to known external doses of the chemicals (i.e. forward dosimetry), and they can also be used to extrapolate in vitro toxicity data to external dose values which is referred to as reverse dosimetry (Clewell and Clewell III 2008; Louisse et al. 2010; Rietjens et al. 2011). Unlike traditional animal studies used in risk assessment, PBK models are not limited in the use in extrapolation beyond the range of the experimental data and can be developed for different species, exposure routes, durations and doses based on the requirements (Lin and Fisher 2020).

Generally, three types of input parameters are required to set up a PBK model including (1) physiological parameters (e.g. cardiac output, tissue weight/volumes and tissue blood flows), 
(2) physico-chemical parameters (e.g. tissue: blood partition coefficients), and (3) parameters to describe toxicokinetics (e.g. kinetic constants of absorption, metabolism or excretion) (Krewski et al. 2014; Rietjens et al. 2011). The physiological parameters are used to describe the anatomy of the compartments and their interconnection via blood, being the skeleton of a PBK model. These compartments are defined to represent the most relevant target organs and tissues for the chemical and the toxicological endpoint of interest. Extensive information on anatomical and physiological parameters are available in the literature for different species (Brown et al. 1997; Kapraun et al. 2019). The physico-chemical and kinetic parameters are chemical-specific parameters, and they can be obtained from the literature and/or using in vitro and in silico approaches. In vivo toxicokinetic studies can also be performed to derive the model parameters, which nowadays is (often) not preferred due to the conflict of in vivo studies with the $3 \mathrm{R}$ principle. Approaches to derive key model parameters are summarized below.

The intestinal absorption of chemicals can be correlated to their permeability measured using an artificial membrane such as in the parallel artificial membrane permeability assay (Fortuna et al. 2012) or using cell-based models such as Caco-2 transport studies (Hubatsch et al. 2007; Skolnik et al. 2010; Strikwold et al. 2017a). Moreover, absorption related kinetic parameters can also be predicted using physicochemical, permeability and solubility data using commercial software programs where physiologically based dynamic absorption models are included (Jamei et al. 2009; Matsumura et al. 2020). The distribution of a chemical into tissue compartments and the systemic circulation can be described by the tissue: blood or plasma partition coefficients. These partition coefficients can be derived from experimental methods using biological tissues and equilibration techniques (Fisher et al. 2020b; Gargas et al. 1989; Jepson et al. 1994). Many mathematic algorithms have been developed to describe the tissue partitioning of a chemical to organs (Berezhkovskiy 2004; DeJongh et al. 1997; Rodgers et al. 2005; Rodgers and Rowland 2006; Schmitt 2008). Additionally, these algorithms have been incorporated in PBK software packages, for example, PK-SIM, Simcyp, and GastroPlus (Fisher et al. 2020b). Metabolic kinetic parameters can be determined using various in vitro systems and then be extrapolated to the in vivo situation by using scaling factors. These in vitro models include incubations with recombinant enzymes (e.g. recombinant CYPs), tissue fractions (e.g. hepatic S9 fraction, microsomes and cytosols), cell models (e.g. primary cells and hiPSC) with respective applicability domains (Fisher et al. 2020a). Some of these assays such as the ones using microsomes and recombinant metabolic enzymes, have been applied to study the human variability in the metabolism of chemicals (Boonpawa et al. 2017; Ning et al. 2019; Strikwold 
et al. 2017a). Traditionally, urinary excretion can be determined based on the cumulative mass of the chemical excreted in the urine in in vivo studies (Fisher et al. 2020b). More recently, transfected cells with transporter proteins and 3D-culture of primary cells are developed to determine the kinetics of biliary and urinary excretion and the corresponding scaling factors (Cheng et al. 2016; Noorlander et al. 2021a and b; Qiao et al. 2021).

\subsection{PBK modelling-based reverse dosimetry}

PBK models have been widely used in chemical risk assessment for several purposes, such as evaluation of interactions between different compounds (Alhusainy et al. 2010; Dennison et al. 2004; Tan et al. 2011), investigation of the influence of age and physiological variations on dosimetry (Ning et al. 2019; Strikwold et al. 2017b; Yang et al. 2006; Yang et al. 2019) and facilitating the use of in vitro toxicity models for a quantitative risk assessment (Louisse et al. 2010; Rietjens et al. 2011; Wetmore et al. 2012; Yoon et al. 2012). One good example of the latter application is so-called PBK modelling-based reverse dosimetry that translates in vitro concentration-response curves to predicted in vivo dose-response curves from which points of departure (PoDs) can be derived to define safe human exposure levels of chemicals (Louisse et al. 2010; Rietjens et al. 2011; Yoon et al. 2012; Zhao et al. 2019). In the present thesis this approach was applied for human cardiotoxicity induced by the two anti-addictive model compounds.

The PBK modelling-based reverse dosimetry approach is schematically presented in Figure 6 and proceeds by several steps as follows. The first step is to select an appropriate in vitro cardiotoxicity model from which concentration-response curves can be derived. The second step is the development of a PBK model for the model compound and its metabolites in human using kinetic parameters obtained from in vitro models and parameters derived from in silico simulations and/or the literature. The third step is to evaluate the performance of the developed model. For this purpose, comparisons are made between predicted kinetics (e.g. time course of blood or plasma concentration and area under the blood or plasma concentration time curve) and experimental kinetics reported in clinical studies. The model evaluation can be complemented with a sensitivity analysis which is required to identify influential parameters on the model predictions, and can be used to better interpret the model predictions and may also be used to select the parameters that need to be estimated with the highest accuracy (Barton et al. 2007; Covington and Gearhart 2020). Once the performance of the model has been adequately evaluated, the model can be used for reverse dosimetry as described in the next step. The fourth step is to translate in vitro concentration-response curves obtained in the first step 
to in vivo dose-response curves. To do this the in vitro effective concentration of the compound or its metabolite of interest is set equal to the relevant internal concentration (i.e. concentration in the heart venous blood in the present thesis). Ideally this extrapolation should be based on the unbound fraction of both the in vitro concentrations and the internal concentration to eliminate the potential influence of differences in binding in the in vitro and in vivo situation (e.g. binding to medium components and well plate plastic, and protein binding to human plasma) on bioavailability of the chemical or its metabolite in both the in vitro model and the in vivo situation. The extrapolation is applied for each in vitro concentration to obtain an entire dose-response curve from which PoDs such as the no observed adverse effect level (NOAEL), the benchmark dose (BMD) or an associated lower bound confidence limit (BMDL), can be derived for the risk assessment and safety evaluation of the compound. The last step is to evaluate the predictions made by the in vitro-PBK modelling-based reverse dosimetry approach by comparing predicted dose-response data and $\mathrm{PoD}$ values to data obtained from in vivo studies reported in the literature.

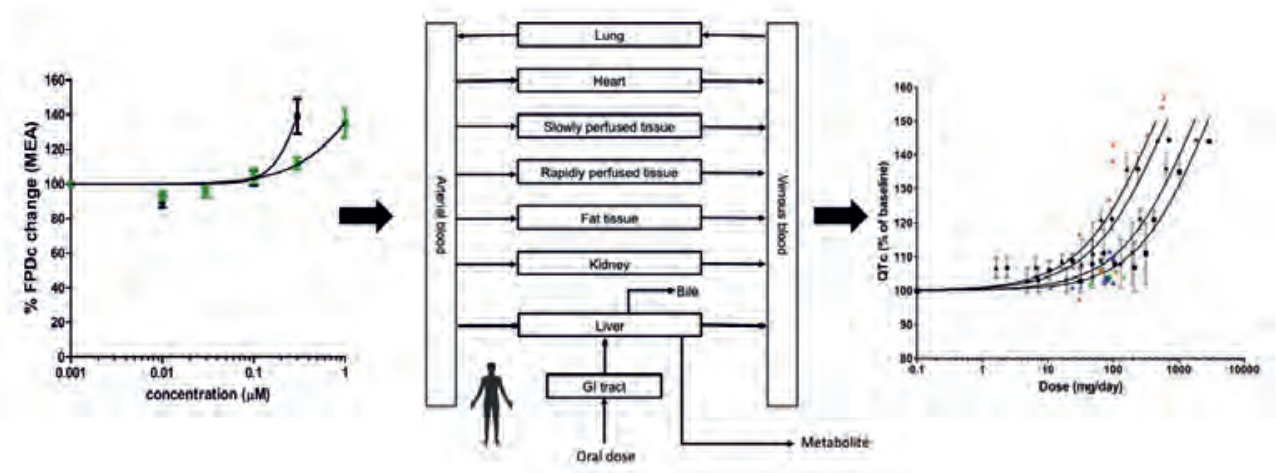

Figure 6 Principle of the physiologically based kinetic (PBK) modelling-based reverse dosimetry approach

\subsection{Use of Monte Carlo simulation in variability analysis}

By combining with Monte Carlo (MC) simulation, PBK modelling can be used to explore the inter-individual or inter-ethnic variations in the toxicokinetics of chemicals due to the variability in, for example, age, gender, ethnicity, genotype and/or lifestyle (Clewell III and Andersen 1996; Gentry et al. 2002; Gearhart et al. 1993; Ning et al. 2019; Rietjens et al. 2011; Strikwold et al. 2017b; Yang et al. 2006). MC simulation is a statistical technique that involves the random sampling of parameter values, for example for the PBK model, from the distribution(s) of these parameters (e.g. metabolic kinetic parameters) to perform a large 
number of PBK model simulations, subsequently generating the distribution of output parameters of interest, which typically are internal dose metrics (Covington and Gearhart 2020). PBK modelling-based reverse dosimetry combined with MC simulation can, in addition to the PBK model development and evaluation, be performed following several steps (Krishnan et al. 2013; Ning et al. 2019; Strikwold et al. 2017b). (1) Selection of the input model parameters of interest for the MC simulation and predictions. Usually the selected parameters show large variability among the population and are highly influential to the model output based on results of the sensitivity analysis, i.e. metabolic constants in the present thesis. (2) Defining the distribution of the parameters relevant for the MC analysis and selecting distribution parameters including i.e. the geometric mean (GM) and standard deviations, which can be obtained from experiments and/or from literature. Generally, physiological parameters such as body volume, are considered to have a normal distribution while physico-chemical and kinetic parameters including partition coefficients and metabolic constants are considered to have log-normal distributions (Clewell et al. 1999; Clewell et al. 2004; Covington et al. 2007; Zhang et al. 2007). (3) Running an MC simulation to randomly sample the desired number of input parameters from their respective distributions. (4) PBK modelling to predict the distribution of the output parameter of interest using the MC simulation. (5) Statistical analysis of the distribution of the output generated in (4), i.e. derivation of GM (geometric mean), $95^{\text {th }}, 97.5^{\text {th }}$ and $99^{\text {th }}$ percentiles from the distribution which can be used to define a chemical-specific adjustment factor (CSAF) that can replace the default uncertainty factor used in risk assessment to account for human inter-individual differences in toxicokinetics (International Programme on Chemical Safety (IPCS), 2005).

\section{Outline of thesis}

Chapter 1 as the introduction chapter starts with the background information on alternative test strategies/ NAM and the aim of the present PhD project. It also provides a definition on cardiotoxicity, followed by the summaries of toxicokinetic and toxicodynamic profiles of the two model compounds used for the studies and their metabolites. Subsequently the main approaches applied in the present project, including two in vitro cardiotoxicity assays, PBK modelling and Monte Carlo simulations are introduced. Chapter $\mathbf{2}$ evaluates a mouse and a human stem cell-based in vitro model, namely the mESC-CM beating arrest assay and the hiPSC-CM MEA assay, for cardiotoxicity screening of chemicals. Eleven cardiotoxic chemicals with different modes of action were used as reference compounds. The results obtained from the two models were compared to each other and to in vivo cardiotoxicity data, 
to provide insight into their applicability domains and to enable selection of a suitable toxicity assay for QIVIVE in subsequent chapters. In Chapter 3 the cardiotoxicity of methadone and its metabolites EDDP and EMDP were quantified using the hiPSC-CM MEA assay. A PBK model of racemic methadone was developed to enable the translation of the in vitro concentration-response curve obtained to an in vivo dose-response curve for methadoneinduced QTc prolongation. The outcomes were compared with available human in vivo QTc prolongation data to evaluate the model performance. As follow-up of the work described in Chapter 3, Chapter 4 investigated the potential of the developed QIVIVE approach for the prediction of human inter-individual variability in in vivo cardiotoxicity of methadone. To this end in vitro cardiotoxicity and metabolic data were integrated with PBK models and Monte Carlo simulations to predict the effect of inter-individual and inter-ethnic kinetic variations on the cardiotoxicity of the two methadone enantiomers in the Caucasian and the Chinese population. CSAFs were defined and used to derive dose-response curves for the sensitive individuals. The kinetic variations obtained using individual human liver microsomes or recombinant cytochrome P450 enzymes (rCYPs) were compared. Chapter 5 investigates whether PBK modeling-based reverse dosimetry of in vitro data was able to adequately predict the human cardiotoxicity of the herbal alkaloid ibogaine and its metabolite noribogaine. The TEQ approach was incorporated in the PBK model enabling the evaluation of the role of noribogaine in ibogaine-induced in vivo cardiotoxicity. Chapter 6 summarizes the results obtained in the thesis, provides an overall discussion and presents the future perspectives that follow from the results obtained. 


\section{Reference}

Abassi YA, Xi B, Li N, et al. (2012) Dynamic monitoring of beating periodicity of stem cell-derived cardiomyocytes as a predictive tool for preclinical safety assessment. British journal of pharmacology 165(5):1424-1441

Abdullah R, Alhusainy W, Woutersen J, et al. (2016) Predicting points of departure for risk assessment based on in vitro cytotoxicity data and physiologically based kinetic (PBK) modeling: the case of kidney toxicity induced by aristolochic acid I. Food and Chemical Toxicology 92:104-116

Abramson FP (1982) Methadone plasma protein binding: Alterations in cancer and displacement from $\alpha 1$-acid glycoprotein. Clinical Pharmacology \& Therapeutics 32(5):652-658

Algharably EAe-H, Di Consiglio E, Testai E, et al. (2021) Prediction of the dose range for adverse neurological effects of amiodarone in patients from an in vitro toxicity test by in vitro-in vivo extrapolation. Archives of Toxicology:1-10

Alhusainy W, Paini A, Punt A, et al. (2010) Identification of nevadensin as an important herb-based constituent inhibiting estragole bioactivation and physiology-based biokinetic modeling of its possible in vivo effect. Toxicology and Applied Pharmacology 245(2):179-190

Alinejad S, Kazemi T, Zamani N, et al. (2015) A systematic review of the cardiotoxicity of methadone. EXCLI journal 14:577

Alper K (2001) Ibogaine: A Review. The Alkaloids Chemistry and biology 56:1-38

Alper K, Bai R, Liu N, et al. (2016) hERG blockade by iboga alkaloids. Cardiovascular toxicology 16(1):14-22

Andersen ME, McMullen PD, Phillips MB, et al. (2019) Developing context appropriate toxicity testing approaches using new alternative methods (NAMs). ALTEX-Alternatives to animal experimentation 36(4):523-534

Ansermot N, Albayrak Ö, Schläpfer J, et al. (2010) Substitution of (R, S)-methadone by (R)-methadone: impact on QTc interval. Archives of internal medicine 170(6):529-536

Asua I (2013) Growing menace of ibogaine toxicity. British journal of anaesthesia 111(6):1029-1030

Bailey J, Thew M, Balls M (2014) An analysis of the use of animal models in predicting human toxicology and drug safety. Alternatives to Laboratory Animals 42(3):181-199

Barbosa Neto JO, Garcia MA, Garcia JBS (2015) Revisiting methadone: pharmacokinetics, pharmacodynamics and clinical indication. Revista Dor 16(1):60-66

Barton HA, Chiu WA, Woodrow Setzer R, et al. (2007) Characterizing uncertainty and variability in physiologically based pharmacokinetic models: state of the science and needs for research and implementation. Toxicological Sciences 99(2):395-402

Baumann MH, Pablo J, Ali SF, Rothman RB, Mash DC (2001a) Comparative neuropharmacology of ibogaine and its O-desmethyl metabolite, noribogaine.

Baumann MH, Rothman RB, Pablo JP, Mash DC (2001b) In vivo neurobiological effects of ibogaine and its Odesmethyl metabolite, 12-hydroxyibogamine (noribogaine), in rats. Journal of Pharmacology and Experimental Therapeutics 297(2):531-539

Behzadi M, Joukar S, Beik A (2018) Opioids and cardiac arrhythmia: a literature review. Medical principles and practice $27(5): 401-414$ 
Bell SM, Chang X, Wambaugh JF, et al. (2018) In vitro to in vivo extrapolation for high throughput prioritization and decision making. Toxicology In Vitro 47:213-227

Berezhkovskiy LM (2004) Determination of volume of distribution at steady state with complete consideration of the kinetics of protein and tissue binding in linear pharmacokinetics. Journal of pharmaceutical sciences 93(2):364-374

Bernauer U, Oberemm A, Madle S, Gundert-Remy U (2005) The use of in vitro data in risk assessment. Basic \& clinical pharmacology \& toxicology 96(3):176-181

Bil W, Zeilmaker M, Fragki S, Lijzen J, Verbruggen E, Bokkers B (2021) Risk assessment of per-and polyfluoroalkyl substance mixtures: A relative potency factor approach. Environmental Toxicology and Chemistry 40(3):859-870

Blaauboer BJ (2010) Biokinetic modeling and in vitro-in vivo extrapolations. Journal of Toxicology and Environmental Health, Part B 13(2-4):242-252

Boonpawa R, Spenkelink A, Punt A, Rietjens IMCM (2017) In vitro-in silico-based analysis of the dose-dependent in vivo oestrogenicity of the soy phytoestrogen genistein in humans. British journal of pharmacology 174(16):2739-2757

Brown RP, Delp MD, Lindstedt SL, et al. (1997) Physiological parameter values for physiologically based pharmacokinetic models. Toxicology and industrial health 13(4):407-484

Burnett SD, Blanchette AD, Chiu WA, Rusyn I (2021) Human induced pluripotent stem cell (iPSC)-derived cardiomyocytes as an in vitro model in toxicology: strengths and weaknesses for hazard identification and risk characterization. Expert Opinion on Drug Metabolism \& Toxicology:1-16

Burridge PW, Li YF, Matsa E, et al. (2016) Human induced pluripotent stem cell-derived cardiomyocytes recapitulate the predilection of breast cancer patients to doxorubicin-induced cardiotoxicity. Nature medicine 22(5):547

Chang YX, Mummery CL (2018) Human-induced pluripotent stem cell-derived cardiomyocytes in the evaluation of cardiotoxic potential of drugs Stem Cell Genetics for Biomedical Research. Springer, p 173-194

Cheng Y, Woolf TF, Gan J, He K (2016) In vitro model systems to investigate bile salt export pump (BSEP) activity and drug interactions: A review. Chemico-biological interactions 255:23-30

Chiu WA, Barton HA, DeWoskin RS, et al. (2007) Evaluation of physiologically based pharmacokinetic models for use in risk assessment. Journal of Applied Toxicology: An International Journal 27(3):218-237

Clewell HJ, Gearhart JM, Gentry PR, et al. (1999) Evaluation of the uncertainty in an oral reference dose for methylmercury due to interindividual variability in pharmacokinetics. Risk Analysis 19(4):547-558

Clewell HJ, Gentry PR, Covington TR, et al. (2004) Evaluation of the potential impact of age-and gender-specific pharmacokinetic differences on tissue dosimetry. Toxicological Sciences 79(2):381-393

Clewell III HJ, Andersen ME (1996) Use of physiologically based pharmacokinetic modeling to investigate individual versus population risk. Toxicology 111(1-3):315-329

Clewell RA, Clewell III HJ (2008) Development and specification of physiologically based pharmacokinetic models for use in risk assessment. Regulatory Toxicology and Pharmacology 50(1):129-143

Covington TR, Gearhart JM (2020) Sensitivity and Monte Carlo analysis techniques and their use in uncertainty, variability, and population analysis Physiologically Based Pharmacokinetic (PBPK) Modeling. Elsevier, p 211-242 
Covington TR, Gentry PR, Van Landingham CB, et al. (2007) The use of Markov chain Monte Carlo uncertainty analysis to support a Public Health Goal for perchloroethylene. Regulatory Toxicology and Pharmacology 47(1):1-18

Dale O, Sheffels P, Kharasch ED (2004) Bioavailabilities of rectal and oral methadone in healthy subjects. British journal of clinical pharmacology 58(2):156-162

Davis AK, Barsuglia JP, Windham-Herman A-M, et al. (2017) Subjective effectiveness of ibogaine treatment for problematic opioid consumption: short-and long-term outcomes and current psychological functioning. Journal of psychedelic studies 1(2):65-73

De Vos J, Ufkes J, van Wilgenburg H, et al. (1995) Pharmacokinetics of methadone and its primary metabolite in 20 opiate addicts. European journal of clinical pharmacology 48(5):361-366

Dean M (2004) Opioids in renal failure and dialysis patients. Journal of pain and symptom management 28(5):497504

DeJongh J, Verhaar HJ, Hermens JL (1997) A quantitative property-property relationship (QPPR) approach to estimate in vitro tissue-blood partition coefficients of organic chemicals in rats and humans. Archives of Toxicology $72(1): 17-25$

Dennison JE, Andersen ME, Dobrev ID, et al. (2004) PBPK modeling of complex hydrocarbon mixtures: gasoline. Environmental Toxicology and Pharmacology 16(1-2):107-119

Doherty KR, Wappel RL, Talbert DR, et al. (2013) Multi-parameter in vitro toxicity testing of crizotinib, sunitinib, erlotinib, and nilotinib in human cardiomyocytes. Toxicology and applied pharmacology 272(1):245-255

Dunlop J, Bowlby M, Peri R, et al. (2008) High-throughput electrophysiology: an emerging paradigm for ionchannel screening and physiology. Nature reviews Drug discovery 7(4):358-368

Eap CB, Buclin T, Baumann P (2002) Interindividual variability of the clinical pharmacokinetics of methadone. Clinical pharmacokinetics 41(14):1153-1193

Eap CB, Crettol S, Rougier JS, et al. (2007) Stereoselective block of hERG channel by (S)-methadone and QT interval prolongation in CYP2B6 slow metabolizers. Clinical Pharmacology \& Therapeutics 81(5):719728

Eap CB, Cuendet C, Baumann P (1990) Binding of d-methadone, 1-methadone, and dl-methadone to proteins in plasma of healthy volunteers: Role of the variants of $\alpha 1$-acid glycoprotein. Clinical Pharmacology \& Therapeutics 47(3):338-346

European Chemicals Agency (ECHA) (2016) New Approach Methodologies in Regulatory Science, Proceedings of a scientific workshop Helsinki, 19-20 April 2016, ECHA-16-R21-EN

European Commission (2007) Corrigendum to Regulation (EC) No 1907/2006 of the European Parliament and of the Council of 18 December 2006 concerning the Registration, Evaluation, Authorisation and Restriction of Chemicals (REACH), establishing a European Chemicals Agency, amending Directive 1999/45/EC and repealing Council Regulation (EEC) No 793/93 and Commission Regulation (EC) No 1488/94 as well as Council Directive 76/769/EEC and Commission Directives 91/155/EEC, 93/67/EEC, 93/105/EC and 2000/21/EC. Official Journal of the European Union L136: 3-280

European Commission (2008) Regulation (EC) No 1333/2008 of the European parliament and of the council of 16 December 2008 on food additives. Official Journal of the European Union 354:16-33

European Commission (2009) Regulation (EC) No 1223/2009 of the European parliament and of the council of 30 November 2009 on cosmetic products. Official Journal of the European Union L342:59-209 
European Commission (2010a) Directive 2010/63/EU of the European Parliament and of the Council of 22 September 2010 on the protection of animals used for scientific purposes. Official Journal of the European Union L276: 53: 33-79.

European Commission (2010b) Directive 2010/84/EU of the European parliament and of the council of 15 December 2010 amending, as regards pharmacovigilance, Directive 2001/83/EC on the Community code relating to medicinal products for human use. Official Journal of the European Union L348:74-99

European Food Safety Authority (EFSA) (2013). International frameworks dealing with human risk assessment of combined exposure to multiple chemicals. Efsa Journal, 11(7), 3313.

European Food Safety Authority (EFSA) (2019). Guidance on harmonised methodologies for human health, animal health and ecological risk assessment of combined exposure to multiple chemicals. Efsa journal, 17(3).

European Medicines Agency (EMA) 2017 Guideline on the principles of regulatory acceptance of 3Rs (replacement, reduction, refinement) testing approaches.

Evans MJ, Kaufman MH (1981) Establishment in culture of pluripotential cells from mouse embryos. nature 292(5819):154-156

Ewart L, Gallacher DJ, Gintant G, et al. (2012) How do the top 12 pharmaceutical companies operate safety pharmacology? Journal of pharmacological and toxicological methods 66(2):66-70

Fareed A, Vayalapalli S, Scheinberg K, et al. (2013) QTc interval prolongation for patients in methadone maintenance treatment: a five years follow-up study. The American journal of drug and alcohol abuse 39(4):235-240

Fermini B, Fossa AA (2003) The impact of drug-induced QT interval prolongation on drug discovery and development. Nature reviews Drug discovery 2(6):439-447

Fisher JW, Campbell Jr JL, Lin Z (2020a) Metabolism and physiologically based pharmacokinetic models Physiologically Based Pharmacokinetic (PBPK) Modeling. Elsevier, p 161-173

Fisher JW, Yang X, Mehta D, et al. (2020b) Fundamentals of physiologically based pharmacokinetic modeling Physiologically Based Pharmacokinetic (PBPK) Modeling. Elsevier, p 57-80

Fortuna A, Alves G, Soares-Da-Silva P, Falcão A (2012) Optimization of a parallel artificial membrane permeability assay for the fast and simultaneous prediction of human intestinal absorption and plasma protein binding of drug candidates: application to dibenz $[\mathrm{b}, \mathrm{f}]$ azepine-5-carboxamide derivatives. Journal of pharmaceutical sciences 101(2):530-540

Foster DJ, Somogyi AA, Bochner F (1999) Methadone N-demethylation in human liver microsomes: lack of stereoselectivity and involvement of CYP3A4. British journal of clinical pharmacology 47(4):403-412

G Postema P, AM Wilde A (2014) The measurement of the QT interval. Current cardiology reviews 10(3):287294

Garg P, Garg V, Shrestha R, et al. (2018) Human induced pluripotent stem cell-derived cardiomyocytes as models for cardiac channelopathies: a primer for non-electrophysiologists. Circulation research 123(2):224-243

Gargas ML, Burgess RJ, Voisard DE, et al. (1989) Partition coefficients of low-molecular-weight volatile chemicals in various liquids and tissues. Toxicology and applied pharmacology 98(1):87-99

Gearhart J, Mahle D, Greene R, et al. (1993) Variability of physiologically based pharmacokinetic (PBPK) model parameters and their effects on PBPK model predictions in a risk assessment for perchloroethylene (PCE). Toxicology letters 68(1-2):131-144 
Gentry PR, Hack CE, Haber L, et al. (2002) An approach for the quantitative consideration of genetic polymorphism data in chemical risk assessment: examples with warfarin and parathion. Toxicological sciences 70(1):120-139

Gerber JG, Rhodes RJ, Gal J (2004) Stereoselective metabolism of methadone N-demethylation by cytochrome P4502B6 and 2C19. Chirality 16(1):36-44

Gilbert-Sandoval I, Wesseling S, Rietjens IMCM (2020) Predicting the Acute Liver Toxicity of Aflatoxin B1 in Rats and Humans by an In Vitro-In Silico Testing Strategy. Molecular nutrition \& food research 64(13):2000063

Glue P, Cape G, Tunnicliff D, et al. (2016) Ascending single-dose, double-blind, placebo-controlled safety study of noribogaine in opioid-dependent patients. Clinical pharmacology in drug development 5(6):460-468

Glue P, Lockhart M, Lam F, et al. (2015a) Ascending-dose study of noribogaine in healthy volunteers: Pharmacokinetics, pharmacodynamics, safety, and tolerability. The Journal of Clinical Pharmacology 55(2):189-194

Glue P, Winter H, Garbe K, et al. (2015b) Influence of CYP2D6 activity on the pharmacokinetics and pharmacodynamics of a single $20 \mathrm{mg}$ dose of ibogaine in healthy volunteers. The Journal of Clinical Pharmacology 55(6):680-687

Grimm D (2019) EPA plan to end animal testing splits scientists. American Association for the Advancement of Science

Grogan J, Gerona R, Snow JW, Kao L (2019) Ibogaine Consumption With Seizure-Like Episodes, QTcProlongation, and Captured Cardiac Dysrhythmias. The Journal of emergency medicine 57(4):e99-e104

Hamill OP, Marty A, Neher E, et al. (1981) Improved patch-clamp techniques for high-resolution current recording from cells and cell-free membrane patches. Pflügers Archiv 391(2):85-100

Hanna AD, Lam A, Tham S, et al. (2014) Adverse effects of doxorubicin and its metabolic product on cardiac RyR2 and SERCA2A. Molecular pharmacology 86(4):438-449

Harris K, Aylott M, Cui Y, et al. (2013) Comparison of electrophysiological data from human-induced pluripotent stem cell-derived cardiomyocytes to functional preclinical safety assays. toxicological sciences $134(2): 412-426$

Hildyard C, Macklin P, Prendergast B, Bashir Y (2016) A case of QT prolongation and torsades de pointes caused by ibogaine toxicity. Journal of Emergency Medicine 50(2):e83-e87

Himmel HM (2013) Drug-induced functional cardiotoxicity screening in stem cell-derived human and mouse cardiomyocytes: effects of reference compounds. Journal of pharmacological and toxicological methods 68(1):97-111

Hoelen DW, Spiering W, Valk GD (2009) Long-QT syndrome induced by the antiaddiction drug ibogaine. New England journal of medicine 360(3):308-309

Hondeghem L, De Clerck F (2012) Preclinical cardiovascular safety evaluations of biologics. BioDrugs 26(5):275282

Huang CL-H (2017) Murine electrophysiological models of cardiac arrhythmogenesis. Physiological reviews 97(1):283-409

Hubatsch I, Ragnarsson EG, Artursson P (2007) Determination of drug permeability and prediction of drug absorption in Caco-2 monolayers. Nature protocols 2(9):2111 
Interagency Coordinating Committee on the Validation of Alternative Methods (ICCVAM) (2018). A Strategic Roadmap for Establishing New Approaches to Evaluate the Safety of Chemicals and Medical Products in the United States. https://dx.doi.org/10.22427/NTP-ICCVAM-ROADMAP2018.

International Programme on Chemical Safety (IPCS) (2005). Chemical-specific adjustment factors for interspecies differences and human variability: guidance document for use of data in dose/ concentration-response assessment. WHO, Geneva. https://apps.who.int/iris/bitstream/handle/10665/43294/9241546786_eng.pdf?sequence=1\&isAllowed= $\mathrm{y}$

Inturrisi CE, Colburn WA, Kaiko RF, et al. (1987) Pharmacokinetics and pharmacodynamics of methadone in patients with chronic pain. Clinical Pharmacology \& Therapeutics 41(4):392-401

Irvine JD, Takahashi L, Lockhart K, et al. (1999) MDCK (Madin-Darby canine kidney) cells: a tool for membrane permeability screening. Journal of pharmaceutical sciences 88(1):28-33

Jamei M, Marciniak S, Feng K, et al. (2009) The Simcyp ${ }^{\circledR}$ population-based ADME simulator. Expert opinion on drug metabolism \& toxicology 5(2):211-223

Jeevaratnam K, Chadda KR, Huang CL-H, Camm AJ (2018) Cardiac potassium channels: physiological insights for targeted therapy. Journal of cardiovascular pharmacology and therapeutics 23(2):119-129

Jepson GW, Hoover DK, Black RK, et al. (1994) A partition coefficient determination method for nonvolatile chemicals in biological tissues. Fundamental and Applied Toxicology 22(4):519-524

Justo D, Gal-Oz A, Paran Y, Goldin Y, Zeltser D (2006) Methadone-associated Torsades de Pointes (polymorphic ventricular tachycardia) in opioid-dependent patients. Addiction 101(9):1333-1338

Kannankeril P, Roden DM, Darbar D (2010) Drug-induced long QT syndrome. Pharmacological reviews 62(4):760-781

Kapraun DF, Wambaugh JF, Setzer RW, Judson RS (2019) Empirical models for anatomical and physiological changes in a human mother and fetus during pregnancy and gestation. PloS one 14(5):e0215906

Karakikes I, Ameen M, Termglinchan V, Wu JC (2015) Human induced pluripotent stem cell-derived cardiomyocytes: insights into molecular, cellular, and functional phenotypes. Circulation research 117(1):80-88

Kharasch ED, Walker A, Whittington D, et al. (2009) Methadone metabolism and clearance are induced by nelfinavir despite inhibition of cytochrome P4503A (CYP3A) activity. Drug and alcohol dependence 101(3):158-168

Kitaguchi T, Moriyama Y, Taniguchi T, et al. (2017) CSAHi study: detection of drug-induced ion channel/receptor responses, QT prolongation, and arrhythmia using multi-electrode arrays in combination with human induced pluripotent stem cell-derived cardiomyocytes. Journal of pharmacological and toxicological methods 85:73-81

Koenig X, Kovar M, Boehm S, et al. (2014) Anti-addiction drug ibogaine inhibits hERG channels: a cardiac arrhythmia risk. Addiction biology 19(2):237-239

Kontrimavičiūte V, Mathieu O, Mathieu-Daudé J-C, et al. (2006) Distribution of ibogaine and noribogaine in a man following a poisoning involving root bark of the Tabernanthe iboga shrub. Journal of analytical toxicology 30(7):434-440

Kratz JM, Grienke U, Scheel O, et al. (2017) Natural products modulating the hERG channel: heartaches and hope. Natural product reports 34(8):957-980 
Krewski D, Westphal M, Andersen ME, et al. (2014) A framework for the next generation of risk science. Environmental health perspectives 122(8):796-805

Krishna S, Berridge B, Kleinstreuer N (2020) High-Throughput Screening to Identify Chemical Cardiotoxic Potential. Chemical Research in Toxicology

Krishnan K, McPhail B, Chiu W, White P (2013) Modeling of Sensitive Subpopulations and Interindividual Variability in Pharmacokinetics for Health Risk Assessments Computational Toxicology. Elsevier, p 45 66

Kuryshev YA, Kirsch GE, Brown AM (2010) Increased cardiac risk in concomitant methadone and diazepam treatment: pharmacodynamic interactions in cardiac ion channels. Biophysical Journal 98(3):339a

Li J, Hua Y, Miyagawa S, et al. (2020) hiPSC-Derived Cardiac Tissue for Disease Modeling and Drug Discovery. International Journal of Molecular Sciences 21(23):8893

Li X, Zhang R, Zhao B, et al. (2016) Cardiotoxicity screening: a review of rapid-throughput in vitro approaches. Archives of toxicology 90(8):1803-1816

Li Y, Kantelip J-P, Gerritsen-van Schieveen P, Davani S (2008) Interindividual variability of methadone response. Molecular diagnosis \& therapy 12(2):109-124

Lin Z, Fisher JW (2020) A history and recent efforts of selected physiologically based pharmacokinetic modeling topics Physiologically Based Pharmacokinetic (PBPK) Modeling. Elsevier, p 1-26

Litjens RP, Brunt TM (2016) How toxic is ibogaine? Clinical Toxicology 54(4):297-302

Louisse J, de Jong E, van de Sandt JJ, et al. (2010) The use of in vitro toxicity data and physiologically based kinetic modeling to predict dose-response curves for in vivo developmental toxicity of glycol ethers in rat and man. Toxicological Sciences 118(2):470-484

Lugo RA, Satterfield KL, Kern SE (2005) Pharmacokinetics of methadone. Journal of Pain \& Palliative Care Pharmacotherapy 19(4):13-24

Ma J, Guo L, Fiene SJ, et al. (2011) High purity human-induced pluripotent stem cell-derived cardiomyocytes: electrophysiological properties of action potentials and ionic currents. American Journal of PhysiologyHeart and Circulatory Physiology 301(5):H2006-H2017

Maltsev VA, Wobus AM, Rohwedel J, et al. (1994) Cardiomyocytes differentiated in vitro from embryonic stem cells developmentally express cardiac-specific genes and ionic currents. Circulation research 75(2):233244

Martin RL, McDermott JS, Salmen HJ, et al. (2004) The utility of hERG and repolarization assays in evaluating delayed cardiac repolarization: influence of multi-channel block. Journal of cardiovascular pharmacology 43(3):369-379

Mash DC, Ameer B, Prou D, et al. (2016) Oral noribogaine shows high brain uptake and anti-withdrawal effects not associated with place preference in rodents. Journal of Psychopharmacology 30(7):688-697

Mash DC, Duque L, Page B, Allen-Ferdinand K (2018) Ibogaine detoxification transitions opioid and cocaine abusers between dependence and abstinence: clinical observations and treatment outcomes. Frontiers in pharmacology 9:529

Mash DC, Kovera CA, Pablo J, et al. (2001) Ibogaine in the treatment of heroin withdrawal.

Matsumura N, Hayashi S, Akiyama Y, et al. (2020) Prediction characteristics of oral absorption simulation software evaluated using structurally diverse low-solubility drugs. Journal of pharmaceutical sciences 109(3):1403-1416 
Miller JM, Meki MH, Ou Q, et al. (2020) Heart slice culture system reliably demonstrates clinical drug-related cardiotoxicity. Toxicology and Applied Pharmacology 406:115213

Moody DE, Alburges ME, Parker RJ, Collins JM, Strong JM (1997) The Involvement of Cytochrome P450 3A4 in theN-Demethylation ofl- $\alpha$-Acetylmethadol (LAAM), norLAAM, and Methadone. Drug Metabolism and Disposition 25(12):1347-1353

National Research Council (2006) Toxicity testing for assessment of environmental agents: interim report. The National Academy Press, Washington, DC

National Research Council (2007) Toxicity testing in the 21st century: a vision and a strategy. The National Academy Press, Washington, DC

Ning J, Rietjens IMCM, Strikwold M (2019) Integrating physiologically based kinetic (PBK) and Monte Carlo modelling to predict inter-individual and inter-ethnic variation in bioactivation and liver toxicity of lasiocarpine. Archives of toxicology 93(10):2943-2960

Noller GE, Frampton CM, Yazar-Klosinski B (2018) Ibogaine treatment outcomes for opioid dependence from a twelve-month follow-up observational study. The American journal of drug and alcohol abuse 44(1):3746

Noorlander A, Fabian E, van Ravenzwaay B, Rietjens IMCM (2021a) Novel testing strategy for prediction of rat biliary excretion of intravenously administered estradiol-17 $\beta$ glucuronide. Archives of Toxicology 95(1):91-102

Noorlander A, Wesseling S, Rietjens IMCM, van Ravenzwaay B (2021b) Incorporating renal excretion via the OCT2 transporter in physiologically based kinetic modelling to predict in vivo kinetics of mepiquat in rat. Toxicology Letters 343:34-43

Nozaki Y, Honda Y, Watanabe H, et al. (2017) CSAHi study-2: validation of multi-electrode array systems (MEA60/2100) for prediction of drug-induced proarrhythmia using human iPS cell-derived cardiomyocytes: assessment of reference compounds and comparison with non-clinical studies and clinical information. Regulatory Toxicology and Pharmacology 88:238-251

O'Connell CW, Gerona RR, Friesen MW, Ly BT (2015) Internet-purchased ibogaine toxicity confirmed with serum, urine, and product content levels. The American journal of emergency medicine 33(7):985. e5985. e6

Obach RS, Pablo J, Mash DC (1998) Cytochrome P4502D6 catalyzes the O-demethylation of the psychoactive alkaloid ibogaine to 12-hydroxyibogamine. Drug metabolism and disposition 26(8):764-768

Olsen GD (1973) Methadone binding to human plasma proteins. Clinical Pharmacology \& Therapeutics 14(3):338-343

Omwenga I, Zhao S, Kanja L, et al. (2021) Prediction of dose-dependent in vivo acetylcholinesterase inhibition by profenofos in rats and humans using physiologically based kinetic (PBK) modeling-facilitated reverse dosimetry. Archives of Toxicology:1-15

Ou Q, Jacobson Z, Abouleisa RR, et al. (2019) Physiological biomimetic culture system for pig and human heart slices. Circulation research 125(6):628-642

Ovics P, Regev D, Baskin P, et al. (2020) Drug Development and the Use of Induced Pluripotent Stem CellDerived Cardiomyocytes for Disease Modeling and Drug Toxicity Screening. International Journal of Molecular Sciences 21(19):7320

Paling F, Andrews L, Valk G, Blom H (2012) Life-threatening complications of ibogaine: three case reports. drugs $1(2)$ 
Pang L, Sager P, Yang X, et al. (2019) Workshop report: FDA workshop on improving cardiotoxicity assessment with human-relevant platforms. Circulation research 125(9):855-867

Perel P, Roberts I, Sena E, et al. (2007) Comparison of treatment effects between animal experiments and clinical trials: systematic review. Bmj 334(7586):197

Priest BT, McDermott JS (2015) Cardiac ion channels. Channels 9(6):352-359

Punt A, Bouwmeester H, Blaauboer BJ, et al. (2020) New approach methodologies (NAMs) for human-relevant biokinetics predictions: Meeting the paradigm shift in toxicology towards an animal-free chemical risk assessment. ALTEX-Alternatives to animal experimentation 37(4):607-622

Qiao S, Feng S, Wu Z, et al. (2021) Functional Proliferating Human Hepatocytes: In Vitro Hepatocyte Model for Drug Metabolism, Excretion, and Toxicity. Drug Metabolism and Disposition 49(4):305-313

Rietjens IMCM, Louisse J, Punt A (2011) Tutorial on physiologically based kinetic modeling in molecular nutrition and food research. Molecular nutrition \& food research 55(6):941-956

Roden DM (2008) Cellular basis of drug-induced torsades de pointes. British journal of pharmacology 154(7):1502-1507

Rodgers T, Leahy D, Rowland M (2005) Physiologically based pharmacokinetic modeling 1: predicting the tissue distribution of moderate-to-strong bases. Journal of pharmaceutical sciences 94(6):1259-1276

Rodgers T, Rowland M (2006) Physiologically based pharmacokinetic modelling 2: predicting the tissue distribution of acids, very weak bases, neutrals and zwitterions. Journal of pharmaceutical sciences 95(6):1238-1257

Romach M, Piafsky K, Abel J, et al. (1981) Methadone binding to orosomucoid ( $\alpha 1$-acid glycoprotein): Determinant of free fraction in plasma. Clinical Pharmacology \& Therapeutics 29(2):211-217

Rougier J-S, Abriel H (2016) Cardiac voltage-gated calcium channel macromolecular complexes. Biochimica Et Biophysica Acta (BBA)-Molecular Cell Research 1863(7):1806-1812

Rovida C, Hartung T (2009) Re-evaluation of animal numbers and costs for in vivo tests to accomplish REACH legislation requirements for chemicals-a report by the transatlantic think tank for toxicology (t4). ALTEX-Alternatives to animal experimentation 26(3):187-208

Rubi L, Eckert D, Boehm S, et al. (2017) Anti-addiction drug ibogaine prolongs the action potential in human induced pluripotent stem cell-derived cardiomyocytes. Cardiovascular toxicology 17(2):215-218

Russell WMS, Burch RL (1959) The principles of humane experimental technique. Methuen

Sanguinetti MC, Jiang C, Curran ME, Keating MT (1995) A mechanistic link between an inherited and an acquird cardiac arrthytmia: HERG encodes the IKr potassium channel. Cell 81(2):299-307

Schep LJ, Slaughter R, Galea S, Newcombe D (2016) Ibogaine for treating drug dependence. What is a safe dose? Drug and alcohol dependence 166:1-5

Schmitt W (2008) General approach for the calculation of tissue to plasma partition coefficients. Toxicology in vitro 22(2):457-467

Siramshetty VB, Nickel J, Omieczynski C, et al. (2016) WITHDRAWN - a resource for withdrawn and discontinued drugs. Nucleic acids research 44(D1):D1080-D1086

Skolnik S, Lin X, Wang J, et al. (2010) Towards prediction of in vivo intestinal absorption using a 96-well Caco2 assay. Journal of pharmaceutical sciences 99(7):3246-3265

Steinberg C, Deyell MW (2018) Cardiac arrest after ibogaine intoxication. Journal of arrhythmia 34(4):455-457 
Strikwold M (2016) Replacing animal experiments in developmental toxicity testing of phenols by combining in vitro assays with physiologically based kinetic (PBK) modelling. Wageningen University

Strikwold M, Spenkelink B, de Haan LH, et al. (2017a) Integrating in vitro data and physiologically based kinetic (PBK) modelling to assess the in vivo potential developmental toxicity of a series of phenols. Archives of toxicology 91(5):2119-2133

Strikwold M, Spenkelink B, Woutersen RA, et al. (2013) Combining in vitro embryotoxicity data with physiologically based kinetic (PBK) modelling to define in vivo dose-response curves for developmental toxicity of phenol in rat and human. Archives of toxicology 87(9):1709-1723

Strikwold M, Spenkelink B, Woutersen RA, et al. (2017b) Development of a combined in vitro physiologically based kinetic (PBK) and Monte Carlo modelling approach to predict interindividual human variation in phenol-induced developmental toxicity. Toxicological sciences 157(2):365-376

Taboureau O, El M'Selmi W, Audouze K (2020) Integrative systems toxicology to predict human biological systems affected by exposure to environmental chemicals. Toxicology and Applied Pharmacology 405:115210

Tan Y-M, Clewell H, Campbell J, Andersen M (2011) Evaluating pharmacokinetic and pharmacodynamic interactions with computational models in supporting cumulative risk assessment. International journal of environmental research and public health 8(5):1613-1630

The International Council for Harmonisation of Technical Requirements for Pharmaceuticals for Human Use (ICH) (2005a) S7B: The non-clinical evaluation of the potential for delayed ventricular re-polarization (QT interval prolongation) by human pharmaceuticals.

Thomas D, Karle C, Kiehn J (2006) The cardiac hERG/IKr potassium channel as pharmacological target: structure, function, regulation, and clinical applications. Current pharmaceutical design 12(18):2271-2283

Totah RA, Allen KE, Sheffels P, et al. (2007) Enantiomeric metabolic interactions and stereoselective human methadone metabolism. Journal of Pharmacology and Experimental Therapeutics 321(1):389-399

Tripathi ON, Ravens U, Sanguinetti MC (2011) Heart rate and rhythm: molecular Basis, pharmacological modulation and clinical implications. Springer Science \& Business Media

U.S. Environmental Protection Agency (EPA) (2000) Supplementary Guidance for Conducting Health Risk Assessment of Chemical Mixtures. ORD.NECA. Washington, DC. EPA/630/R-00/002.

U.S. Environmental Protection Agency (EPA) (2010) Recommended Toxicity Equivalence Factors (TEFs) for Human Health Risk Assessments of 2,3,7,8-Tetrachlorodibenzo-p-dioxin and Dioxin-Like Compounds. ORD.NECA. Washington, DC. EPA/600/R-10/005.

Van Norman GA (2019) Limitations of animal studies for predicting toxicity in clinical trials: is it time to rethink our current approach? JACC: Basic to Translational Science 4(7):845-854

Verebely K, Volavka J, Mulé S, Resnick R (1975) Methadone in man: pharmacokinetic and excretion studies in acute and chronic treatment. Clinical Pharmacology \& Therapeutics 18(2):180-190

Vlaanderen L, Martial L, Franssen E, et al. (2014) Cardiac arrest after ibogaine ingestion. Clinical Toxicology 52(6):642-643

Volpe DA (2011) Drug-permeability and transporter assays in Caco-2 and MDCK cell lines. Future medicinal chemistry 3(16):2063-2077

Walker T, Harris K, Maifoshie E, Chaudhary K (2017) Human Stem Cell-Derived Cardiomyocyte In Vitro Models for Cardiotoxicity Screening. Stem Cells in Toxicology and Medicine:85-121 
Wedam EF, Bigelow GE, Johnson RE, et al. (2007) QT-interval effects of methadone, levomethadyl, and buprenorphine in a randomized trial. Archives of internal medicine 167(22):2469-2475

Wetmore BA, Wambaugh JF, Ferguson SS, et al. (2012) Integration of dosimetry, exposure, and high-throughput screening data in chemical toxicity assessment. Toxicological Sciences 125(1):157-174

Wilkins JN, Ashofteh A, Setoda D, et al. (1997) Ultrafiltration using the Amicon MPS-1 for assessing methadone plasma protein binding. Therapeutic drug monitoring 19(1):83-87

Wobus AM, Wallukat G, Hescheler J (1991) Pluripotent mouse embryonic stem cells are able to differentiate into cardiomyocytes expressing chronotropic responses to adrenergic and cholinergic agents and $\mathrm{Ca} 2+$ channel blockers. Differentiation 48(3):173-182

Wolff K, Rostami-Hodjegan A, Shires S, et al. (1997) The pharmacokinetics of methadone in healthy subjects and opiate users. British journal of clinical pharmacology 44(4):325-334

Yang F, Tong X, McCarver DG, et al. (2006) Population-based analysis of methadone distribution and metabolism using an age-dependent physiologically based pharmacokinetic model. Journal of pharmacokinetics and pharmacodynamics 33(4):485-518

Yang X, Wu H, Mehta D, et al. (2019) Ontogeny equations with probability distributions for anthropomorphic measurements in preterm and term neonates and infants for use in a PBPK model. Computational Toxicology 11:101-117

Yoon M, Campbell JL, Andersen ME, Clewell HJ (2012) Quantitative in vitro to in vivo extrapolation of cellbased toxicity assay results. Critical reviews in toxicology 42(8):633-652

Zhang J, Wilson GF, Soerens AG, et al. (2009) Functional cardiomyocytes derived from human induced pluripotent stem cells. Circulation research 104(4):e30-e41

Zhang X, Tsang AM, Okino MS, et al. (2007) A physiologically based pharmacokinetic/pharmacodynamic model for carbofuran in Sprague-Dawley rats using the exposure-related dose estimating model. Toxicological Sciences 100(2):345-359

Zhao L, Zhang B (2017) Doxorubicin induces cardiotoxicity through upregulation of death receptors mediated apoptosis in cardiomyocytes. Scientific reports 7(1):1-11

Zhao S, Kamelia L, Boonpawa R, et al. (2019) Physiologically based kinetic modeling-facilitated reverse dosimetry to predict in vivo red blood cell acetylcholinesterase inhibition following exposure to chlorpyrifos in the Caucasian and Chinese population. Toxicological sciences 171(1):69-83

Zuppinger C (2019) 3D cardiac cell culture: a critical review of current technologies and applications. Frontiers in cardiovascular medicine $6: 87$

Zuang V, Dura A, Asturiol Bofill D, et al. (2021) Non-animal Methods in Science and Regulation, EUR 30553 EN, Publications Office of the European Union, JRC123531.

Zwartsen A, de Korte T, Nacken P, et al. (2019) Cardiotoxicity screening of illicit drugs and new psychoactive substances (NPS) in human iPSC-derived cardiomyocytes using microelectrode array (MEA) recordings. Journal of molecular and cellular cardiology 136:102-112 


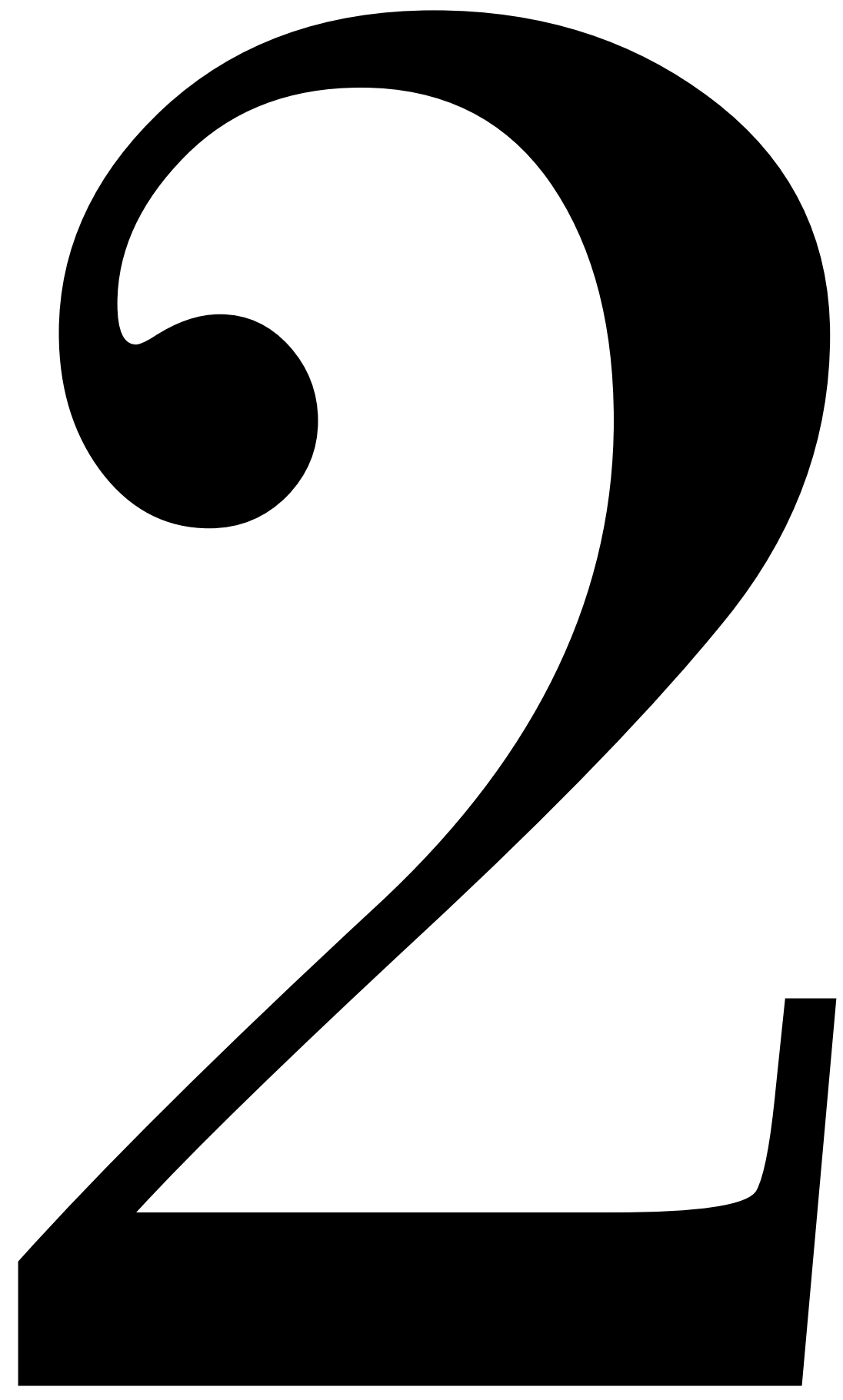




\section{Chapter 2}

\section{Evaluation of in vitro models of stem cell-derived cardiomyocytes to screen for potential cardiotoxicity of chemicals}

Miaoying Shi, Nguyen T. Tien, Laura de Haan, Jochem Louisse, Ivonne M. C. M. Rietjens, Hans Bouwmeester

Published in Toxicology in Vitro (2020), 67, 104891.

https://doi.org/10.1016/j.tiv.2020.104891 


\begin{abstract}
Cardiotoxicity is an important toxicological endpoint for chemical and drug safety assessment. The present study aims to evaluate two stem cell-based in vitro models for cardiotoxicity screening of chemicals. Eleven model compounds were used to evaluate responses of mouse embryonic stem cell-derived cardiomyocytes (mESC-CMs) using beating arrest as a readout and the analysis of electrophysiological parameters measured with a multi-electrode array (MEA) platform of human induced pluripotent stem cell-derived cardiomyocytes (hiPSC-CMs). Results revealed that the hiPSC-CM MEA assay responded to all compounds. The mESC-CM beating arrest assay was not responsive to potassium channel blockers and showed a lower sensitivity to sodium channel blockers and $\mathrm{Na}^{+} / \mathrm{K}^{+}$ATPase inhibitors compared to the hiPSCCM MEA assay. Calcium channel blockers and a $\beta$-adrenergic receptor agonist showed comparable potencies in both models. The in vitro response concentrations from hiPSC-CMs were highly concordant with human effective serum concentrations of potassium and sodium channel blockers. It is concluded that both in vitro models enable the cardiotoxicity screening with different applicability domains. The mESC-CM beating arrest assay may be used as a first step in a tiered approach while the hiPSC-CM MEA assay may be the best starting point for quantitative in vitro to in vivo extrapolations.
\end{abstract}




\section{Introduction}

Cardiotoxicity is considered as an important endpoint in the safety testing of chemicals and drugs. Many promising drug candidates are discontinued during the development because of undesired cardiotoxic effects. In addition, there is an increasing need for the evaluation of foodborne constituents like alkaloids and environmental pollutants that are associated with potential cardiotoxicity (Ainerua et al., 2020; Kratz et al. 2017; Pang et al. 2019; Stevens and Baker 2009). For these reasons the development of new approaches that can quickly and reliably identify and characterize the cardiotoxicity of chemicals would be of a great value. Traditional laboratory animal studies are gradually considered as an inappropriate approach for cardiac safety assessment due to the fact that animal studies are costly, labour intensive and considered unethical (Pang et al. 2019). These considerations promote the development of new technologies where in vitro assays play an important role in characterizing the toxicity of chemicals (Bernauer et al. 2005). The present study aims to evaluate the potential applicability domain of two stem cell-based in vitro models to rapidly screen for the potential cardiotoxicity of chemicals.

Normal cardiac functioning requires cellular ion homeostasis in cardiomyocytes that is maintained by the concerted action of membrane ion channels and ion transporter (Priest and McDermott, 2015; Schwinger et al., 2003). Brief controlled changes in ionic homeostasis lead to changing inward and outward ion fluxes, generating action potentials that ultimately result in the contraction of cardiomyocytes (Rougier and Abriel, 2016; Huang, 2016; Jeevaratnam et al., 2018). Sodium $\left(\mathrm{Na}^{+}\right)$channels are the key drivers for inducing the depolarization of the cell membrane (DeMarco and Clancy, 2016) and calcium channels contribute to maintaining the plateau phase of action potentials (Bers and Perez Reyes, 1999). Various types of potassium $\left(\mathrm{K}^{+}\right)$channels are involved in different phases of repolarization (Priest and McDermott, 2015). In addition to ion channels, several enzymes and transporters such as $\mathrm{Na}^{+} / \mathrm{K}^{+}$ATPase also play critical roles in maintaining the ion homeostasis. Chemical-induced cardiotoxicity is often caused by the off-target interactions with these ion channels and transporters, resulting in aberrant electrophysiological function of cardiomyocytes (Priest and McDermott, 2015). Chemicals induce various types of adverse cardiac events, depending on the affected ion channels or transporters.

Up to date, several in vitro methods are being explored to screen for cardiotoxicity. These models range from reductionistic single ion channel binding studies to technological advanced 
patch clamp techniques that are essential for mechanistic studies. A conventional assay is to measure the inhibitory effect of compounds on individual ion channels. For this, transfected cell lines are used that allow a highly sensitive detection of binding to the target ion channel (Clements and Thomas, 2014). However, this approach fails to address the effects induced by drugs targeting multiple channels (Rehnelt et al., 2017), while also extrapolation to the in vivo situation from transfected cell lines may be dfficult given the differences in expression levels. Models that use the patch clamp technique are considered as the gold standard for detecting cardiotoxicity since it can accurately measure relevant electrophysiological parameters including single ion currents, action potential duration and peak amplitude (Rehnelt et al., 2017). Yet, the patch clamp technique is labour intensive, and the stability of the system is limited due to damage of the cell membrane (Laurila et al., 2016; Tertoolen et al., 2018). Recently, fluorescent imaging techniques have been applied to screen for potential cardiotoxicity of chemicals. For this, voltage-sensitive dyes are used to measure parameters which are comparable to those targeted by the patch clamp technique but without invasive measurement (Laurila et al., 2016). However, this approach is limited by the potential cytotoxicity of these dyes (Chang and Mummery, 2018).

In the past decade, stem cell-derived cardiomyocytes have been integrated as in vitro models in preclinical safety assessments (Pouton and Haynes, 2007; Denning and Anderson, 2008; Kettenhofen and Bohlen, 2008; Freund and Mummery, 2009). Stem cell-derived cardiomyocytes have first been obtained from the mouse embryonic stem cells (Wobus et al., 1991; Maltsev et al., 1994). Mouse embryonic stem cell derived cardiomyocytes (mESC-CMs) express the major cardiac contractile proteins, ion channels and receptors (Abassi et al., 2012; Himmel, 2013), which allow them to serve as comprehensive models to detect the cardiotoxic effect of compounds which target multiple mechanisms. Functional beating cardiomyocytes are obtained easily from mouse embryonic stem cells by spontaneous differentiation, without the need of specific growth factors (Seiler and Spielmann, 2011; Kamelia et al., 2017). Moreover, Nicolas et al. (2015) reported that mESC-CMs can successfully detect in vitro cardiotoxicity of various ion channel blockers, by determining chemical-induced concentration-dependent cardiac beating arrest. This provides a robust and easy to use platform for the detection of cardiotoxicity. Human induced pluripotent stem cell derived cardiomyocytes (hiPSC-CMs) have shown their potential as the in vitro model for cardiotoxicity testing (Freund and Mummery, 2009). HiPSCs do not spontaneously differentiate into functional cardiomyocytes, but require more elaborated culturing techniques, including the application of growth factors in 
the medium (Lewandowski et al., 2017; Sala et al., 2017). HiPSC-CMs express the major cardiac ion channels, receptors, transporters and electrophysiological responses, known to be present in human cardiomyocytes (Ma et al., 2011; Karakikes et al., 2015; Chang and Mummery, 2018; Pouttierand Fedida., 2020). These hiPSC-CMs have often been applied in combination with the multi-electrode array (MEA) techniques, which has proven to be a medium throughput and non-invasive approach for the detection of cardiotoxicity (Harris et al., 2013; Li et al., 2016; Nozaki et al., 2016; Kitaguchi et al., 2017; Ando et al., 2017). By measuring extracellular field potential for monolayers of cardiomyocytes grown on the chip, the MEA technique can characterize several electrophysiological parameters which specifically correspond to the specific phases of the in vivo electrocardiogram (ECG) and can thus be used to correlate the in vitro functional measurements to human in vivo clinical data (Halbach et al., 2003; Sala et al., 2017).

This study aimed to identify the applicability domain of two stem cell-based assays to screen for the potential cardiotoxicity of chemicals. For this we used the mESC-CMs with a simple readout (beating arrest) as a relatively high throughput and low-cost assay and compared it with the lower throughput and high-cost hiPSC-CM MEA assay. Eleven compounds with known mode-of-action of cardiac effects that target potassium channels, calcium channels, sodium channels, $\mathrm{Na}^{+} / \mathrm{K}^{+}$ATPase and $\beta$-adrenergic receptor were tested in both models. The effect concentrations were compared to reported serum concentrations related to in vivo cardiotoxicity obtained from human studies.

\section{Materials and methods}

\subsection{Chemical}

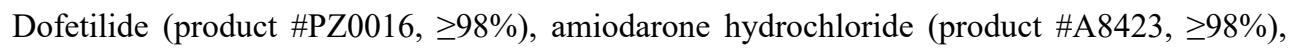
sematilide monohydrochloride monohydrate (product \#S0323, $\geq 98 \%$ ), moxifloxacin

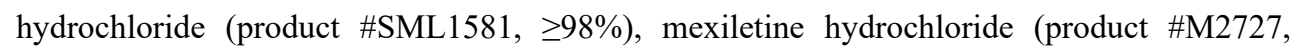
$\geq 98 \%$ ), flecainide acetate salt (product $\# \mathrm{~F} 6777, \geq 98 \%$ ), verapamil hydrochloride (product

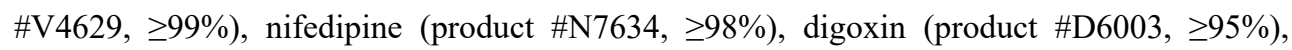
ouabain octahydrate (product $\# \mathrm{O} 3125, \geq 97 \%$ ) and isoproterenol hydrochloride (product \#1351005, $\geq 98 \%$ ) were purchased from Sigma-Aldrich (Zwijndrecht, The Netherlands). Dimethyl sulfoxide (DMSO, >99.7\%) was obtained from Merck (Schiphol-Rijk, The Netherlands). All stock solutions and dilutions of test compounds were prepared in DMSO. 


\subsection{In vitro cardiotoxicity in the $\mathrm{mESC}-\mathrm{CM}$ beating arrest assay}

In the mESC-CM beating arrest assay the in vitro cardiotoxicity was characterised by quantifying the effect of test compounds on the beating of cardiomyocytes formed from the pluripotent mouse embryonic stem cell line D3 (ATCC, Wesel, Germany). The cells were cultured in HyClone AdvanceSTEM ${ }^{\mathrm{TM}}$ Low Osmo Dulbecco's Modified Eagle Medium (DMEM, Fischer Scientific, Landsmeer, The Netherlands) supplemented with $20 \%$ heat inactivated fetal bovine serum (FBS, ATCC, Manassas, USA), $50 \mathrm{U} / \mathrm{ml}$ penicillin (Invitrogen, The Netherlands), $50 \mu \mathrm{g} / \mathrm{ml}$ streptomycin (Invitrogen) and $2 \mathrm{mM} \mathrm{L}$-glutamine (Invitrogen). The cells were cultured at $37{ }^{\circ} \mathrm{C}$ with $5 \% \mathrm{CO}_{2}$ in a humidified atmosphere and subcultured three times per week. Non-enzymatic cell dissociation solution (Sigma-Aldrich) was used to detach cells and 1,000 U/ml murine leukemia inhibiting factor (mLIF, Sigma-Aldrich) was added to prevent spontaneous differentiation. Cells were grown in $25 \mathrm{~cm}^{2}$ flask (Corning, Amsterdam, the Netherland) precoated with $0.1 \% \mathrm{~m} / \mathrm{v}$ gelatine (Sigma-Aldrich).

To obtain beating cardiomyocytes, the differentiation process of cells was performed according to previously published protocols (Nicolas et al., 2015; Kamelia et al., 2017) with minor modifications. On day $0,20 \mu 1$ cell droplets containing $3.75 \times 10^{4}$ cells $/ \mathrm{ml}$ were hung on the lid of 96-well plates (Greiner BioOne, Alphen a/d Rijn, The Netherlands). Phosphate buffered saline (PBS, Invitrogen) was added to all wells of the 96-well plate to provide humidity and prevent evaporation of the hanging drops. After 3 days incubation at $37{ }^{\circ} \mathrm{C}$ and $5 \% \mathrm{CO}_{2}$, the embryonic bodies formed were transferred to a $60 \times 15 \mathrm{~mm}$ bacteriological petri dish (Greiner Bio-One) containing $5 \mathrm{ml}$ medium and incubated for 3 days. On day 5, embryonic bodies were transferred to 48 -well plates (Greiner Bio-One) (one embryonic body/well). The 48-well plates were incubated at $37{ }^{\circ} \mathrm{C}$ and $5 \% \mathrm{CO}_{2}$ for another 5 days and the cardiomyocytes started beating from day 10 onwards. On day 11, contracting cardiomyocytes were treated with compounds to detect the cardiotoxicity. For each concentration of test compounds, ten wells containing beating cardiomyocytes (10 beating embryonic bodies; 1/well) were exposed and the number of well containing beating embryonic bodies after one-hour incubation with test compound was counted by visual inspection under the microscope. After this visual inspection all wells were washed with fresh medium and incubated for one hour in medium without added test compounds to determine the recovery of beating in the cardiomyocytes. $0.25 \%$ DMSO was used as solvent control. 


\subsection{In vitro cardiotoxicity in the hiPSC-CM MEA assay}

The MEA technology of Multi Channel System (MCS GmbH, Ruetlingen, Germany) was used to assess the field potentials generated by hiPSC-CMs (Pluricyte ${ }^{\circledR}$ Cardiomyocytes) obtained from Ncardia (Leiden, The Netherlands). The cells were prepared according to the manufacturer's protocol. Briefly, cells were thawed in the incubator at $37^{\circ} \mathrm{C}$ for exactly $4 \mathrm{~min}$ and gently transferred to a $50 \mathrm{ml}$ tube. The vial was rinsed with $1 \mathrm{ml}$ serum free Pluricyte ${ }^{\circledR}$ Cardiomyocyte Medium (Ncardia) added drop-wise to the tube containing the cardiomyocytes. Then an additional $5 \mathrm{ml}$ medium were added drop-wise to the tube. $20 \mu \mathrm{l}$ of the homogenous cell suspension thus obtained were taken for manual cell counting using a Buerker-Tuerk Counting Chamber (Marienfeld Superior GmbH \& Co. KG, Lauda-Königshofen, Germany). At the same time cells were centrifuged at $300 \mathrm{~g}$ for 3 minutes. Then the supernatant was removed and medium was drop-wisely added to reach the aimed concentration of cells in the suspension $\left(2 \times 10^{4}\right.$ cells $\left./ 2 \mu \mathrm{l}\right)$. Cells were placed on the 6-well MEA chips (60-6well MEA200/30iR-Ti-tcr) from the Multi Channel System (MCS GmbH) at the concentration of $2 \times 10^{4}$ cells $/ 2 \mu 1 /$ well. Each well was precoated with fibronectin (Sigma-Aldrich) before seeding. MEA chips were incubated at $37^{\circ} \mathrm{C}$ with $5 \% \mathrm{CO}_{2}$ and refreshed with medium every 2 days.

Electrically coupled monolayers of hiPSC-CMs with spontaneous beating behaviour can be obtained 7-8 days post-seeding. MEA chips containing the hiPSC-CMs were placed on the headstage of a MEA2100-System (MCS GmbH) for signal selection. Only the wells with a signal showing clearly visible depolarization and repolarization peaks were selected for further assessment (Sala et al., 2017). As indicated in Figure 1, a typical extracellular field potential waveform consists of a rapid upstroke corresponding to depolarization, a slow wave/plateau and a repolarization peak. Prior to the measurement, MEA chips containing the cells were equilibrated for at least $20 \mathrm{~min}$ in the chamber of the MEA system which provided a stable atmosphere at $37{ }^{\circ} \mathrm{C}$ with $5 \% \mathrm{CO}_{2}$. Then, cells were exposed to increasing concentrations of the model compounds in a cumulative manner as follows: after an equilibration period, DMSO $(0.2 \%)$ was added into the well by replacing half of original medium to reach a final concentration of $0.1 \%$ DMSO. Then test compound was cumulatively added to the well with increasing concentrations in the same way. Including the baseline condition $(0.1 \% \mathrm{DMSO})$, seven concentrations of each compound were tested. Test compounds were diluted from stock solutions into medium to reach the aimed final concentrations. The final concentration of DMSO in exposure medium was kept at $0.1 \%$. At each concentration of test compounds, the extracellular field potential was recorded for $1 \mathrm{~min}$ after $10 \mathrm{~min}$ exposure. Data were collected 
using Cardio 2D software (MCS GmbH) with a sample frequency of $10 \mathrm{kHZ}$ and a $0.1-3.5 \mathrm{kHz}$ band-pass filter.

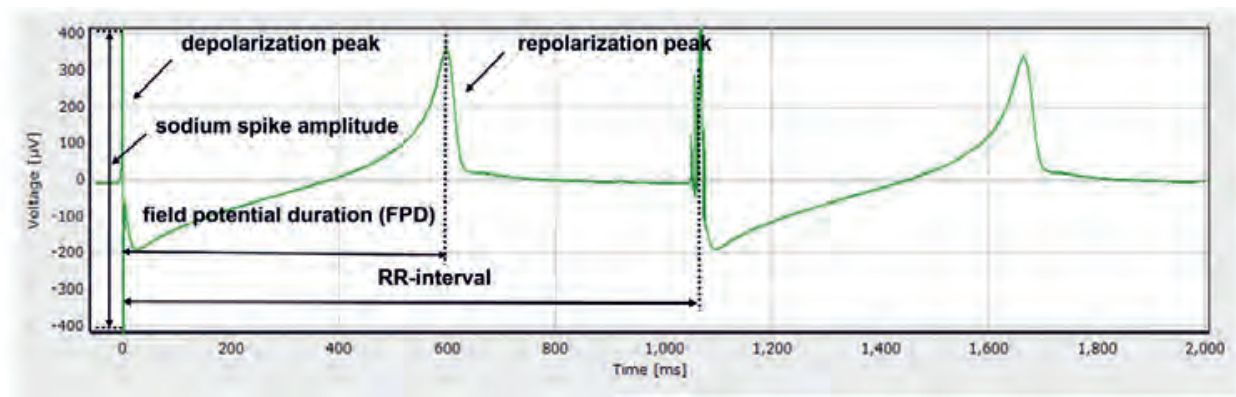

Fig. 1 Typical waveforms of the extracellular field potential signal generated by human cardiomyocytes derived from human induced pluripotent stem cells cultured in 6-well MEA-chips.

\subsection{Data analysis}

In the mESC-CM beating arrest assay, the cardiotoxicity of model compounds was presented as the percentage of wells containing beating cells compared to the solvent control condition for which the response was set at $100 \%$. The concentration-response curves for amiodarone, sematilide, verapamil, digoxin and ouabain were obtained from our previous study (Nicolas et al., 2015). Concentrations with a recovery less than 50\% (1 $\mu \mathrm{M}$ verapamil, $1 \mu \mathrm{M}$ nifedipine and $600 \mu \mathrm{M}$ ouabain, data not shown) were excluded from concentration-response curves obtained from the $\mathrm{mESC}-\mathrm{CM}$ beating arrest assay to minimize the risk that arrest was elicited by general cytotoxicity instead of the reversible interaction with cardiac ion channels. Data represent the mean of at least three independent experiments.

MEA data were analysed using Multiwell-Analyzer software Version 1.5.1.0 (MCS GmbH). Only the electrodes showing a stable field potential trace (Fig. 2) were selected for analysis. The following parameters were measured as the average of at least 30 beats from one-minute recording of each concentration of the compounds (Fig. 2): sodium spike amplitude (defined as the absolute amplitude of the depolarization peak), field potential duration (FPD, defined as the duration between the beginning of the sodium spike and the repolarizing peak) and RR-interval (the duration between two depolarization peaks). To correct the effect of beat rate on FPD, the clinically used Fridericia 's formula was applied (Vandenberk et al., 2016), which is commonly used in cardiotoxicity-related studies (Ando et al., 2017; Kitaguchi et al., 2017): 


$$
\mathrm{FPDc}=\frac{\mathrm{FPD}}{\sqrt[3]{\mathrm{RR} \text { interval }}}
$$

In this formula the FPD and RR-interval were expressed in seconds. Beat per minute (BPM) was derived from RR-intervals, being the duration between two depolarization peaks:

$$
\mathrm{BPM}=\frac{60}{\mathrm{RR} \text { interval }}
$$

The RR-interval was expressed in seconds. Concentrations that induced arrhythmia-like changes in the waveform and/or beating arrests were excluded from analysis of these parameters since the FPD, RR-interval and sodium spike could not be determined (Kitaguchi et al., 2016; Zwartsen et al., 2019). Data were collected from at least three independent experiments (3-7 wells, 11-37 electrodes). Results are expressed as relative percentage compared to the results obtained for the baseline control $(0.1 \%$ DMSO). The response of baseline control was set at $100 \%$. The target ion channels or receptors and relevant endpoints of the compounds are summarized in Table 1.

The benchmark dose (BMD) approach was applied on the in vitro concentration-response curves obtained from both assays to derive the benchmark concentrations. A $10 \%$ change in the readouts (beating arrest for $\mathrm{mESC}-\mathrm{CMs}$ and electrical activity for hiPSC-CMs) was used as the benchmark response to calculate the benchmark concentration $\left(\mathrm{BMC}_{10}\right)$ for cardiotoxicity with lower-upper $95 \%$ confidence interval. As the model compounds target different ion channels or receptors, and thus cause different electrophysiological effects in the hiPSC-CM MEA assay, $\mathrm{BMC}_{10}$ values were expressed in a mode-of-action specific way (see Results).

BMD analysis was performed using the European Food Safety Authority (EFSA) web-tool ${ }^{1}$ for BMD analysis based on the R-package PROAST version 66.40 developed by the Dutch National Institute for Public Health and the Environment (RIVM). Model selection and model fitting was performed according to the flow-chart described in the manual provided by EFSA ${ }^{1}$. Briefly, the quantal data obtained from the MESC-CM beating arrest assay were fitted using the available quantal models including (Log)-logistic, (Log)-probit, Weibull, Gamma, two-stage, Exponential and Hill model. The continuous data from the hiPSC-CM MEA assay were fitted to a set of models including Exponential, Hill, Inverse Exponential model and Log-Normal Family. Analysis was performed according to the flow-chart described in the manual ${ }^{1}$. All fitted models excluding FULL and NULL were used for model averaging described in Wheeler and Bailer (2007) where a weighted average model was constructed to estimate model averaged

1 EFSA Statistical Models-BMD. [Online]. Available at: https://shiny-efsa.openanalytics.eu/app/bmd [Accessed August 1, 2019] 
confidence intervals using bootstrap sampling. Weighting was based the model's Akaike's Information Criterion (AIC) values where models with lower AIC values count larger weight. The final BMC confidence intervals from model average were based on 200 bootstrap data sets. The final $\mathrm{BMC}_{10}$ values were obtained by averaging the model-specific BMC estimates by the following equation as described by Buckland et al. (1997), Bailer et al. (2005) and Wheeler and Bailer (2007):

$$
\widehat{\mathrm{BMC}}=\sum_{\mathrm{k}=1}^{\mathrm{K}} \mathrm{BMC}_{\mathrm{k}} * \omega_{\mathrm{k}}
$$

where $\mathrm{BMC}_{\mathrm{k}}$ is estimated based on the accepted model $\mathrm{k}$ and $\omega_{\mathrm{k}}$ represents the corresponding weight for the model $\mathrm{k}$. Detailed information on the BMD analysis of in vitro data can be found in the supplementary materials (Tab. S1-S17).

The concentration response curves obtained from both in vitro assays were plotted with Graph Pad Prism 5.0 (GraphPad Software Inc., San Diego, USA). Each data point is presented as the mean value \pm standard error of the mean (SEM). Statistical significance was analysed by one-way ANOVA followed by post Dunnett test. Values of $p<0.05$ were regarded as statistically significant. Statistical analysis was performed by Graph Pad Prism 5.0 (GraphPad Software Inc.).

\subsection{Comparison of in vitro and in vivo human cardiotoxicity}

To further evaluate the sensitivity of the hiPSC-CM MEA assay the in vitro response concentrations were compared with reported internal effect concentrations related to human clinical ECG data. For this, the in vitro $\mathrm{BMC}_{10}$ concentrations were compared with unbound human plasma concentrations corresponding to $10 \%$ change on $\mathrm{ECG}\left(\mathrm{hECG}_{10}\right)$. In vivo human data are especially available for the endpoint of the prolongation of the QT interval defined as the prolonged duration between the beginning of ventricular repolarization (QRS complex) and the end of depolarization (T wave) in the ECG, and the change of the QRS complex. Potassium channel blockers increased the in vitro FPDc in the hiPSC-CM MEA assay which can be seen as the surrogate for the QT interval in the ECG (Halbach et al, 2003; Zwartsen et al., 2019). The effect of sodium channel blockers on the sodium spike amplitude in the hiPSC-CM MEA assay was correlated to the change of the QRS complex in the human ECG.

Effective concentrations derived from the hiPSC-CM MEA assay are considered as unbound concentrations due to usage of serum free medium in this assay (Harris et al., 2013). Human ECG data were obtained from published literature (Tab. 1) where the concentration-response 
curves were extracted from graphs using GetData Graph Digitizer $2.26^{2}$ to calculate the $\mathrm{hECG}_{10}$. A zero-effect was included in the dataset, assuming a no effect at a zero compound concentration in serum (in vivo). Obtained $\mathrm{hECG}_{10}$ values were derived from the ECGs using BMD analysis as described for the in vitro data for continuous data. Detailed information on the BMD analysis of in vivo data can be found in the supplementary materials (Tab. S18-S25). The unbound $\mathrm{hECG}_{10}$ values were directly taken from literature when reported or were calculated by multiplying $\mathrm{hECG}_{10}$ values with unbound fraction $\left(\mathrm{f}_{\mathrm{u}}\right)$. The fractions unbound were taken from literature (see Tab. 1).

\section{Results}

\subsection{Screening for cardiotoxic effects using the mESC-CM beating arrest assay}

Dofetilide, amiodarone and sematilide are class III antiarrhythmic agents which inhibit the repaid delayed rectifying potassium current through the (human) Ether-a-go-go Related Gene (ERG) potassium channel. Moxifloxacin is known as an antibiotic but blocks the (h)ERG potassium channel as side effect. These four (h)ERG potassium channel blockers did not significantly inhibit the beating of $\mathrm{mESC}-\mathrm{CMs}$ within the tested concentration ranges (data are shown in Fig. S1A-D in the supplementary data). Figure 2A-B show that the sodium channel blockers, mexiletine and flecainide inhibited the beating of the mESC-CMs in a concentrationdependent manner and induced maximum inhibition at $1,000 \mu \mathrm{M}$ and $300 \mu \mathrm{M}$, respectively. This resulted in a $\mathrm{BMC}_{10}$ value of $85.4 \mu \mathrm{M}$ for mexiletine and $13.4 \mu \mathrm{M}$ for flecainide (Tab. 1). The calcium channel blockers verapamil and nifedipine significantly inhibited the beating of mESC-CMs from $0.1 \mu \mathrm{M}$ and $0.01 \mu \mathrm{M}$ onwards (Fig. 2C-D). The $\mathrm{BMC}_{10}$ value derived from the $\mathrm{mESC}-\mathrm{CM}$ beating arrest assay for verapamil was $68.9 \mathrm{nM}$ while nifedipine was more potent with a $\mathrm{BMC}_{10}$ of $5.9 \mathrm{nM}$ (Tab. 1). Digoxin and ouabain are cardiac glycosides that disturb the intracellular $\mathrm{Na}^{+}$and $\mathrm{K}^{+}$ion balance by inhibiting the $\mathrm{Na}^{+} / \mathrm{K}^{+}$ATPase on the membrane of cardiomyocytes (Guo et al., 2013). As depicted in Figure 2E, no inhibitory effect of digoxin on mESC-CMs was found within the tested concentration range. However, ouabain significantly inhibited the beating of mESC-CMs from $200 \mu \mathrm{M}$ with a BMC $\mathrm{BM}_{10}$ of $170.5 \mu \mathrm{M}$ (Fig. 2F, Tab.1). The $\beta$-adrenergic receptor agonist isoproterenol that is used as an antiarrhythmic drug. Figure

\footnotetext{
${ }^{2}$ Available at: http://getdata-graph-digitizer.com [Accessed May 30, 2019]
} 
$2 \mathrm{G}$ shows that isoproterenol inhibited the beating of cardiomyocytes in a concentrationdependent manner with a $\mathrm{BMC}_{10}$ of $2.3 \mathrm{nM}$.
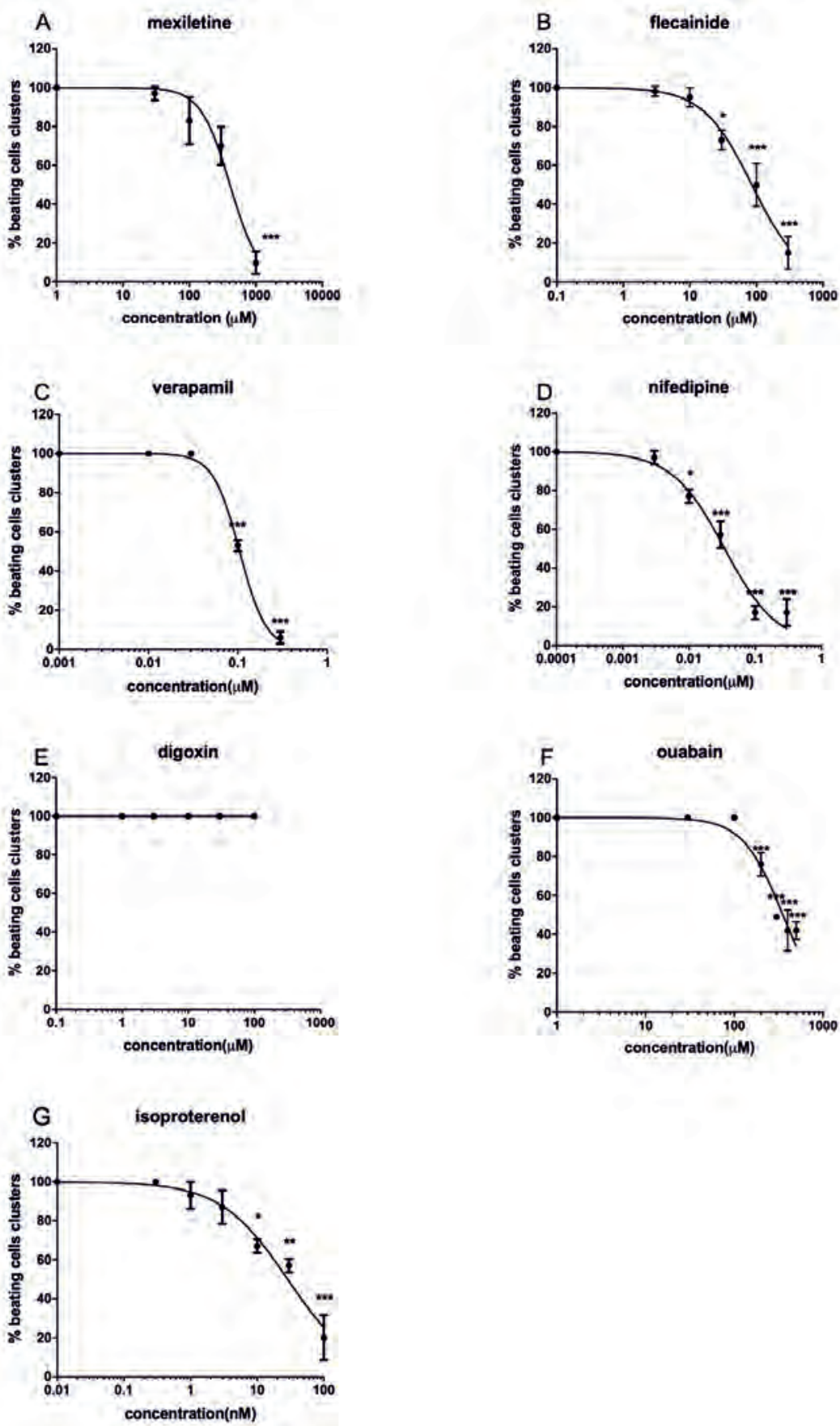
Fig. 2 Concentration-response curves for cardiotoxicity in mESC-CMs of the sodium channel blockers mexiletine (A) and flecainide (B), the calcium channel blockers verapamil (C) and nifedipine (D), the $\mathrm{Na}^{+} / \mathrm{K}^{+}$ATPase inhibitors digoxin (E) and ouabain (F), and the $\beta$-adrenergic receptor agonist isoproterenol (G). The response of the solvent control (DMSO) was set at 100\%. mESC-CMs data represent the mean of at least three independent experiments. Each data point represents the mean \pm SEM. Statistically significant changes compared to the solvent control are marked with * with $p<0.05: *, p<0.01: * *$ and $p<0.001$ : ***.

\subsection{Screening for cardiotoxic effects using the hiPSC-CM MEA assay}

The (h)ERG potassium channel blockers dofetilide, amiodarone, sematilide and moxifloxacin significantly prolonged the FPDc in a concentration-dependent manner and induced $10 \%$ prolongation of the FPDc $\left(\mathrm{FPDc}_{10}\right)$ at $0.86 \mathrm{nM}, 1.6 \mu \mathrm{M}, 0.69 \mu \mathrm{M}$ and $6.5 \mu \mathrm{M}$, respectively (Fig 3A-D, Tab. 1). The results presented in Table 1 also reveal that both arrhythmia-like waveforms and the cessation of beating were observed upon treatment of the hiPSC-CM with dofetilide (at $3 \mathrm{nM}$ and $10 \mathrm{nM}$, respectively) and sematilide (at $1 \mu \mathrm{M}$ and $3 \mu \mathrm{M}$, respectively). Amiodarone caused beating arrest at the highest test concentration of $30 \mu \mathrm{M}$ without inducing arrhythmialike waveforms, while moxifloxacin induced arrhythmia-like waveforms from $30 \mu \mathrm{M}$ onwards but did not induce beating cessation within the tested concentration range (Tab. 1).

Two sodium channel blockers mexiletine and flecainide induced a $10 \%$ reduction of the amplitude (AMP 10 ) at $0.89 \mu \mathrm{M}$ for mexiletine and $0.12 \mu \mathrm{M}$ for flecainide (Fig. 3E-F, Tab. 1). Neither mexiletine nor flecainide induced arrhythmia-like waveforms within the tested concentration ranges while the cessation of beating was observed in most wells at the highest concentrations of mexiletine and flecainide (Tab.1).

For calcium channel blockers, verapamil and nifedipine shortened the FPDc in a concentration-dependent manner (Fig. 3G-H). A 10\% shortening the FPDc (-FPDc 10 ) was observed at a concentration of $4.4 \mathrm{nM}$ for verapamil, and of $13.4 \mathrm{nM}$ for nifedipine (Tab. 1). Verapamil did not induce arrhythmia-like waveforms up to $3 \mu \mathrm{M}$ where complete cessation occurred (Tab. 1). In contrast, nifedipine was not associated with the arrhythmia or beating arrest within the tested concentration range (up to $3 \mu \mathrm{M}$ ).

Figure $3 \mathrm{I}$ and $\mathrm{J}$ show that the $\mathrm{Na}^{+} / \mathrm{K}^{+}$ATPase inhibitors digoxin and ouabain significantly shortened the FPDc in the hiPSC-CM MEA assay with the maximum reduction occurring at comparable concentrations of $1 \mu \mathrm{M}$ and $0.3 \mu \mathrm{M}$, respectively. Arrhythmia-like waveforms were not observed during the exposure of digoxin and ouabain while complete beating cessation 
occurred at the highest tested concentrations of both compounds (Tab. 1). The FPDc 10 was 0.24 $\mu \mathrm{M}$ for digoxin and $0.14 \mu \mathrm{M}$ for ouabain (Tab. 1).

The antiarrhythmic drug isoproterenol increased the beating rate in a concentrationdependent manner with the concentration causing $10 \%$ increase in beating rate $\left(\mathrm{BR}_{10}\right)$ amounting to $5.0 \mathrm{nM}$ (Fig. 3K and Tab. 1). No arrhythmia-like waveforms or beating arrest of hiPSC-CMs was observed up to the highest isoproterenol concentration tested $(30 \mu \mathrm{M})$. 

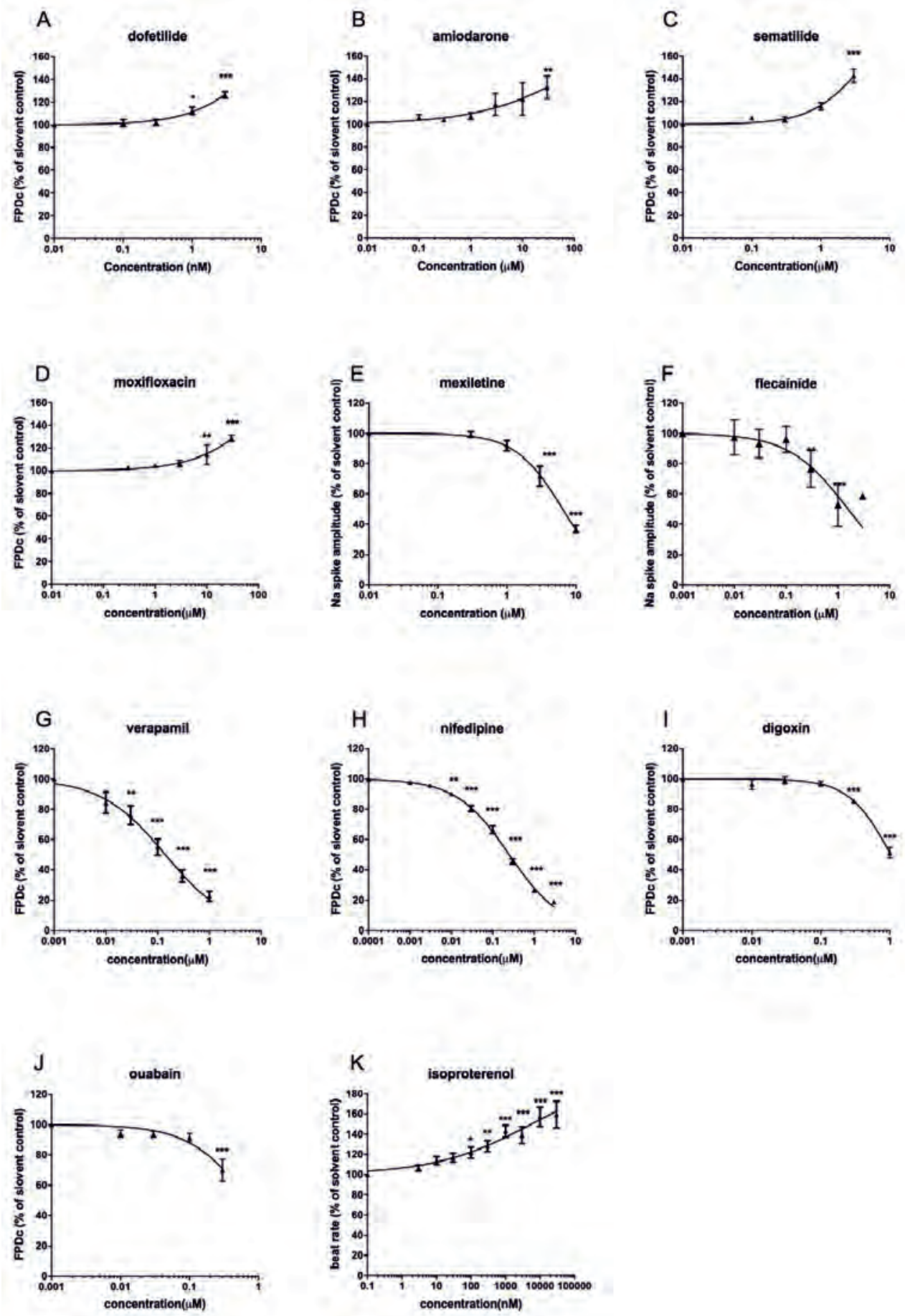
Fig. 3 Concentration-response curves for cardiotoxicity in hiPSC-CMs of the (h)ERG potassium channel blockers dofetilide (A), amiodarone (B), sematilide (C), and moxifloxacin (D), the sodium channel blockers mexiletine $(E)$, and flecainide $(F)$, the calcium channel blockers verapamil (G) and nifedipine (H), the $\mathrm{Na}^{+} / \mathrm{K}^{+}$ATPase inhibitors digoxin (I) and ouabain (J), and the $\beta$-adrenergic receptor agonist isoproterenol (K). The response of the solvent control (DMSO) was set at 100\%. hiPSC-CMs data represent the mean of at least three independent experiments with minimum of eleven electrodes. Each data point represents the mean \pm SEM ( $3 \mu \mathrm{M}$ flecainide is an exception as hiPSC-CMs stopped beating with the exposure of $3 \mu \mathrm{M}$ flecainide and detectable $\mathrm{Na}$ spike amplitude was obtained from 1 out of 7 wells). Statistically significant changes compared to the solvent control are marked with * with $p<0.05: *, p<0.01$ : ** and $p<0.001$ : ***.

\subsection{Comparison of in vitro and in vivo human cardiotoxicity}

Based on the obtained results, the hiPSC-CM MEA assay shows a higher sensitivity and broader compound coverage than the mESC-CM beating arrest assay. Therefore, we next evaluated whether the hiPSC-CM MEA assay provides adequate data to predict human in vivo responses, by comparing the in vitro $\mathrm{FPDc}_{10}$ and $\mathrm{AMP}_{10}$ values for the compounds that induced concentration-dependent changes in these parameters in the hiPSC-CMs, with the unbound $\mathrm{hECG}_{10}$ derived from the related change of waveforms in the ECG from clinical studies (Fig. 4). All available human ECG data are reported in the supplementary file (Tab. S26).

Table 1 and Figure 4 illustrate the comparison for the four (h)ERG potassium channel blockers between their in vitro $\mathrm{FPDc}_{10}$ values and the in vivo unbound $\mathrm{hEC}_{10}$ values, being the unbound plasma concentrations that would prolong the QTc interval by $10 \%$. The FPDc 10 of dofetilide derived from the MEA assay was comparable with its reported unbound $\mathrm{hECG}_{10}$ showing 1.5- to 2.2-fold differences and the FPDc 10 of sematilide was 5-fold lower than the $\mathrm{hECG}_{10}$ value derived from the in vivo data (Tab. 1). For moxifloxacin, the unbound $\mathrm{hECG}_{10}$ $(31.9 \mu \mathrm{M})$ was 5-fold higher than its $\operatorname{FPDc}_{10}$ (Tab. 1), also indicating limited in vitro-in vivo differences. In contrast, the in vitro data for amiodarone were far out of range, resulting in an FPDc $_{10}$ value $(1.6 \mu \mathrm{M})$ that was five orders of magnitude higher than the unbound $\mathrm{hECG}_{10}$ (0.033 nM).

The in vitro-in vivo comparison for the sodium channel blockers mexiletine and flecainide is also shown in Figure 4 and Table 1. The comparison reveals that the $\mathrm{AMP}_{10}$ values obtained in the hiPSC-CM MEA assay were comparable to the unbound $\mathrm{hECG}_{10}$ of mexiletine $(1.6 \mu \mathrm{M})$ (1.8-fold difference) and the range of the unbound $\mathrm{hECG}_{10}$ values reported for flecainide $(0.22$ 
$-0.36 \mu \mathrm{M}$ ) derived from the clinical studies (1.8- to 3- fold difference), indicating an adequate match between in vitro and in vivo human effect concentrations.

For the calcium channel blockers verapamil and nifedipine, the $\mathrm{Na}^{+} / \mathrm{K}^{+}$ATPase inhibitors digoxin and ouabain and the adrenergic receptor antagonist isoproterenol, no adequate human data were available for a comparison between the in vitro data and the in vivo situation. 


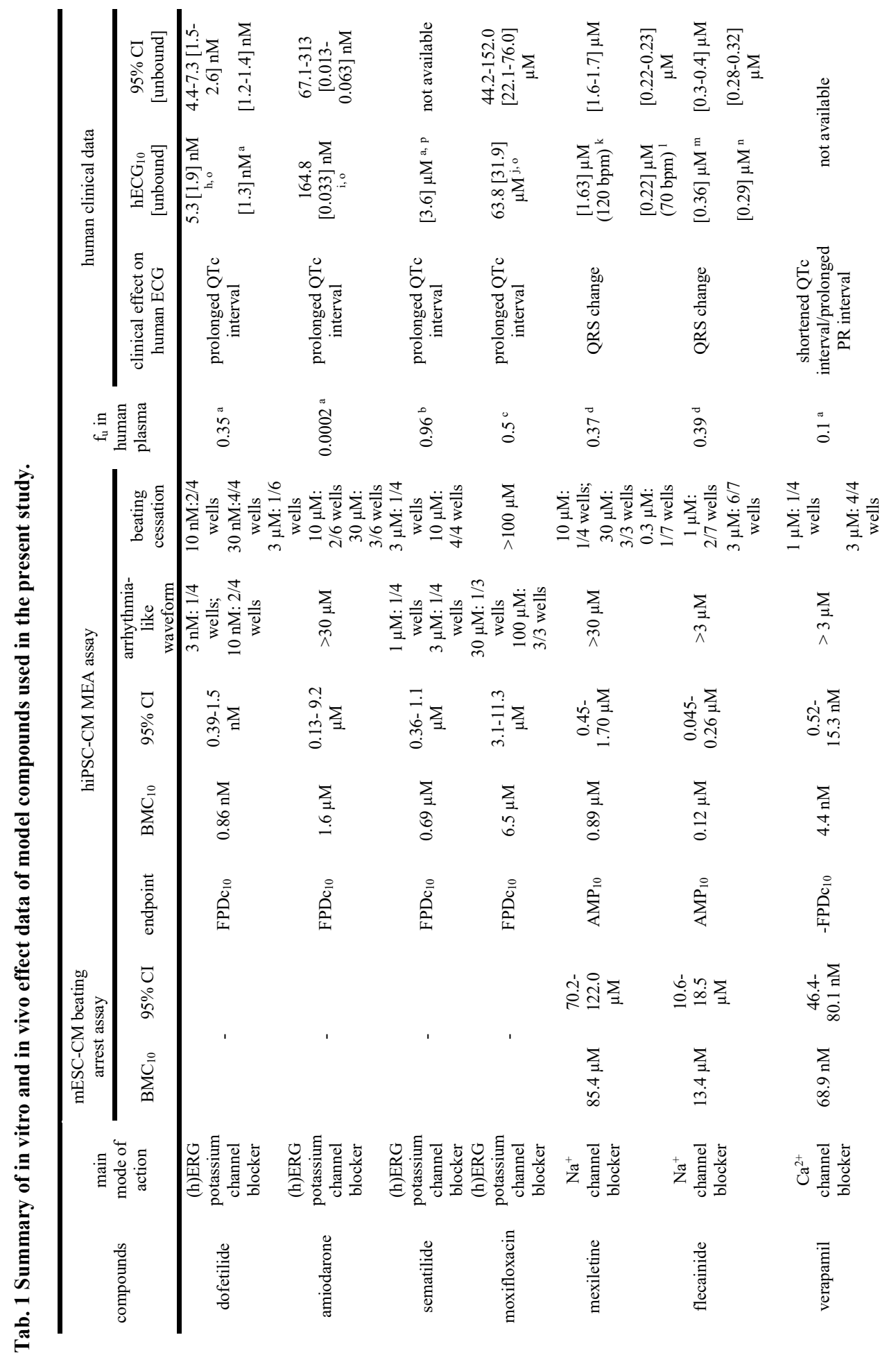




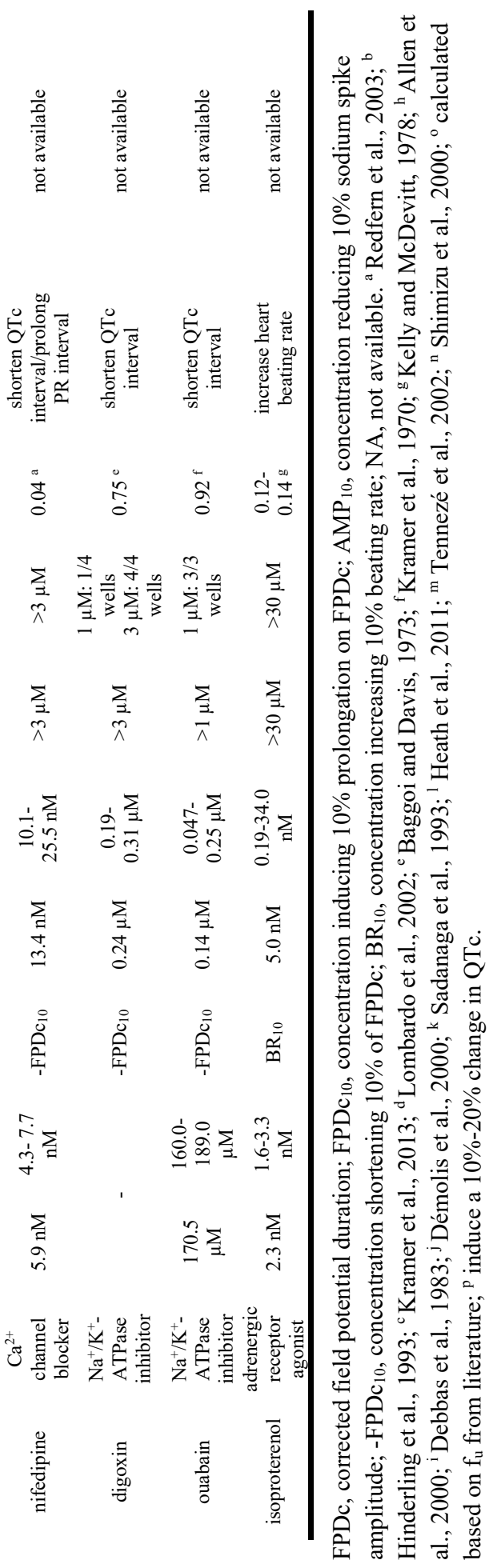




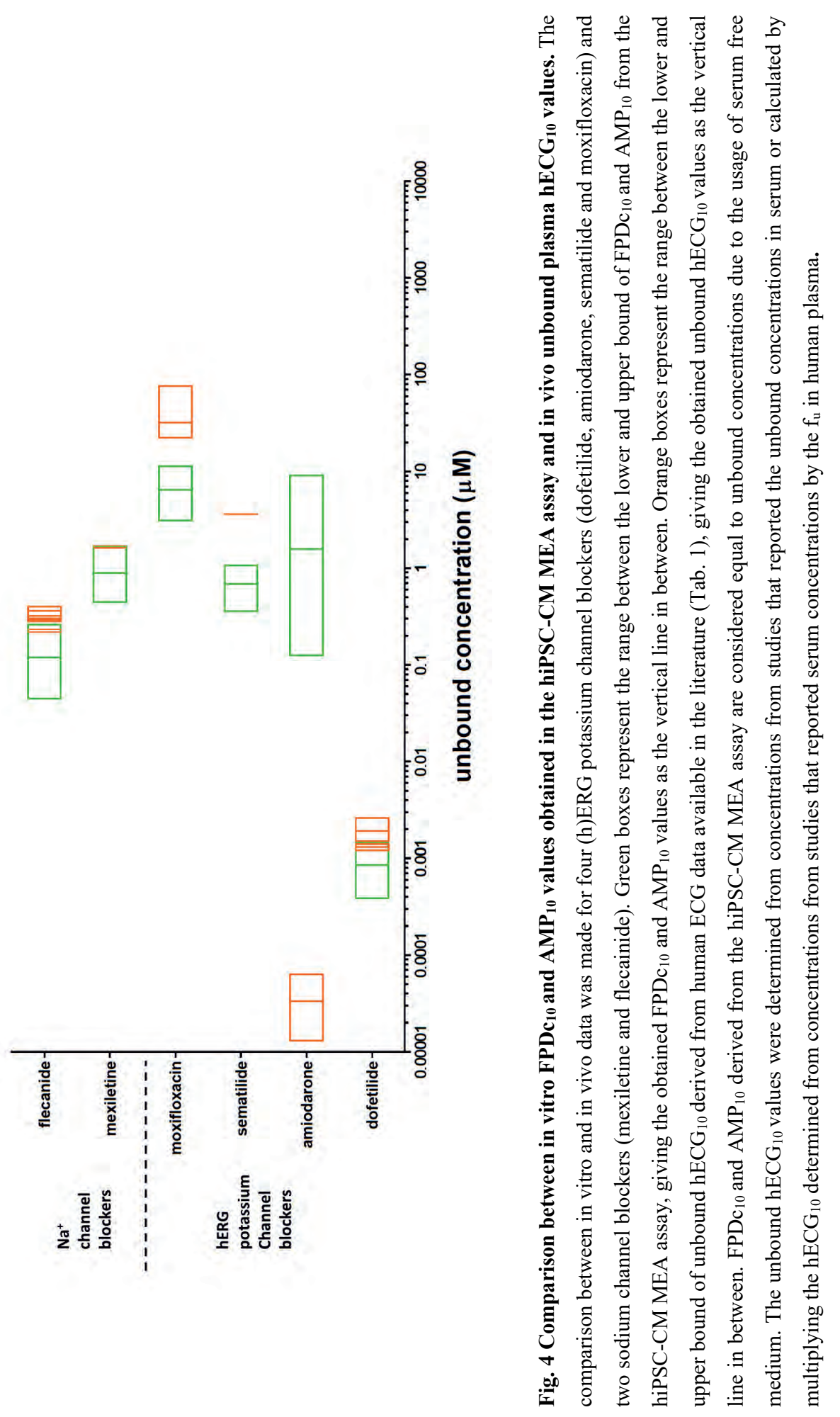




\section{Discussion}

The present study aimed to evaluate use of the mESC-CM beating arrest assay and of the hiPSC-CM MEA assay to screen for the potential cardiotoxicity of chemicals. To evaluate these two models, the effects of eleven model compounds were quantified in both in vitro assays. The in vitro effect concentrations of the hiPSC-CM MEA assay were compared with reported internal effect concentrations related to human clinical ECG data. Based on the obtained results it was concluded that the hiPSC-CM MEA assay is the most versatile assay as it is responsive to all evaluated compounds with a higher sensitivity for (h)ERG potassium and sodium channel blockers and $\mathrm{Na}^{+} / \mathrm{K}^{+}$ATPase inhibitors, with the mESC-CM beating arrest assay being not responsive to (h)ERG potassium channel blockers and to one of the $\mathrm{Na}^{+} / \mathrm{K}^{+}$ ATPase inhibitors. Furthermore, two calcium channel blockers and isoproterenol showed comparable potencies in the two assays. The in vitro effective concentrations obtained from the hiPSC-CM MEA assay correlated well with available in vivo effective concentrations related to human ECG data for (h)ERG potassium and sodium channel blockers.

Given that mESC-CMs are easy to obtain without ethical problems and the mESC-CM beating arrest assay is cost-friendly and easy, requiring less operator skills, the use of the mESC-CM beating arrest assay could be considered as a good first-choice candidate for cardiotoxicity screening. However, from the results obtained in the mESC-CMs, none of the (h)ERG potassium channel blockers induced beating cessation of mESC-CMs within the tested concentration ranges. Corroborating this, similar results (no inhibitory effect) have been found in other studies in which dofetilide and E4031, a typical (h)ERG potassium channel blocker, were both unable to induce the cessation of beating in mESC-CMs (Jonsson et al., 2011; Abassi et al., 2012; Himmel, 2013). No literature data are available on the sodium channel blockers mexiletine and flecainide on mESC-CMs to benchmark our observations. Thus, no comparison could be made. The sodium channel blocker diphenhydramine was reported to induce cessation of beating in mESC-CMs (Nicolas et al., 2015), which is in line with our results showing that sodium channel blockers are active in the $\mathrm{mESC}-\mathrm{CM}$ beating arrest assay. The calcium channel blockers verapamil and nifedipine showed concentration-dependent inhibition of beating, which corroborates results from the study of Himmel (2013) where both compounds induced beating cessation. Digoxin did not induce a response in the mESC-CMs while ouabain inhibited the beating of mESC-CMs at relatively high concentrations $(>100 \mu \mathrm{M})$, which is in line with the study of Himmel (2013) where ouabain failed to induce beating arrest in mESC-CMs at 
concentration from 0.03 to $3 \mu \mathrm{M}$. Isoproterenol was used as a model compound for $\beta$ adrenergic receptor agonists and regulates the cardiac pacemaker action potentials by activating hyperpolarization activated pacemaker channels, further resulting in an increased beating rate (Bers, 2002; Nozaki et al., 2017). An inhibitory effect of isoproterenol on mESCCMs was noted in the present study from the concentration of $1 \mathrm{nM}$ and higher. However, Ikeuchi et al. (2015) did not observe the beating cessation in mESC-CMs up to $1 \mu \mathrm{M}$. A possible reason for the inconsistency between the studies could be related to the various types of cardiac cells in embryonic bodies, resulting in different expression patterns of hyperpolarization activated pacemaker channels. It is reported that these channels are highly expressed in the sinoatrial node cells but low in normal atrial and ventricular cardiomyocytes (Baruscotti et al., 2010; Sartiani et al., 2011).

The hiPSC-CM MEA assay provides insight into the real-time electrophysiological response of compounds in hiPSC-CMs ( $\mathrm{Li}$ et al., 2016). Clearly it is a sensitive and frequently used platform that allows a detection of cardiotoxicity (see supplementary Tab S27; Harris et al., 2013; Nozaki et al., 2016; Nozaki et al., 2017; Kitaguchi et al., 2017; Ando et al., 2017). Most studies have focused on the compounds that target the (h)ERG potassium channels and the current study provides a more comprehensive evaluation of compounds that target other main ion channels and receptors. Our MEA data indicate that all model compounds induced concentration-dependent effects on hiPSC-CMs with the $\mathrm{BMC}_{10}$ values being in accordance with published MEA data showing 1.2- to 5.7-fold differences. In addition, we report concentrations that induce arrhythmia-like waveforms and beating cessation in the same range as obtained from the literature with a maximum 3-fold difference (see references in Tab. S27). Such differences are within the range of inter-laboratory variability of 1.8 - to 20-fold reported by Kitaguchi et al. (2016), Nozaki et al. (2016) and Tamargo et al. (2004).

Both the mESC-CM beating arrest assay and the hiPSC-CM MEA assay can be considered to be functional models to detect cardiotoxicity. However, differences in sensitivity are observed between the two models. The $\mathrm{mESC}-\mathrm{CM}$ beating arrest assay was not responsive to (h)ERG potassium channel blockers and the $\mathrm{Na}^{+} / \mathrm{K}^{+}$ATPase inhibitor digoxin. The hiPSC-CM MEA assay appeared able to detect the effects of all model compounds. Compared with $\mathrm{BMC}_{10}$ values from the $\mathrm{mESC}-\mathrm{CMs}$, the $\mathrm{BMC}_{10}$ obtained from hiPSC-CMs were almost two orders of magnitude lower for the sodium channel blockers and three orders of magnitude lower for the $\mathrm{Na}^{+} / \mathrm{K}^{+}$ATPase inhibitor ouabain. Two calcium channel blockers and isoproterenol showed comparable potencies in the two assays. 
Given the differences obtained between the two assays it is of interest to consider that there are several factors that could explain the distinct sensitivity of the $\mathrm{mESC}-\mathrm{CM}$ beating arrest assay and the hiPSC-CM MEA assay. Although hiPSC-CMs and mESC-CMs express the typical cardiac channels, the expression level and function of these channels are known to be species dependent (Maltsev et al., 1994; Nerbonne, 2004; Jonsson et al., 2011). In the case of potassium channels, the repaid and slow delayed rectifier potassium currents are two predominant currents involved in action potential repolarization in human ventricular cardiomyocytes (Li et al., 1996), while in mouse cardiomyocytes the other three subtypes of delayed rectifier currents (the fast activating and slowly inactivating and steady state current) mainly regulate the repolarization (Xu et al., 1999; Zhou et al., 2003). Thus, all tested (h)ERG potassium channel blockers are only partly involved in the repolarization of the action potential in mouse cardiomyocytes, providing a possible explanation for the lower sensitivity of mESCCMs towards the cardiotoxicity of compounds acing as potassium channel blockers. Furthermore, the maturity of ion channels may also contribute to the differences in sensitivity between the two assays. It has been shown that sodium channels are well-developed at intermediate stage of post-differentiation (15 days after culturing) (Maltsev et al., 1994). While the exposure was performed earlier in our experiments (on day 11), which may have added to the lower sensitivity because of potentially sodium channels present in the mESC-CMs.

In addition, the differentiation level of stem cells appears to be different in the two in vitro models. The hiPSC-CMs protocol results in high purity ventricular cardiomyocytes while a combination of diverse cell types with less than $5 \%$ cardiomyocytes are present in the mESCCMs (Kolossov et al., 2005). This can explain the less extended applicability domain of the mESC-CM beating arrest assay compared to the hiPSC-CM MEA assay. Such variation in the type of cells present upon differentiation of the stem cells could influence the diffusion of compounds to their targets in the cell models as compounds were supposed to have a better diffusion in the monolayer of hiPSC-CMs (Harris et al., 2013), which may result in an apparent lower sensitivity of the mESC-CM beating arrest assay. To add, the lower sensitivity of the mESC-CM beating arrest assay may be in part related to the serum that is present in the exposure medium of the mESC-CMs but not in the hiPSC-CMs medium, potentially reducing the fraction unbound of test compounds. However, given the $f_{u}$ values for binding of test compounds to serum protein (Tab. 1) this could not fully explain the orders of magnitude difference in sensitivity observed. Lastly, the differences in sensitivity could be due to the endpoint that is used in the mESC-CMs. Beating arrest can be regarded as a late cardiac event 
that follows the initial early markers of cardiotoxicity detected by the MEA-related endpoints. For mESC-CMs inactive compounds, including the (h)ERG potassium channel blockers and $\mathrm{Na}^{+} / \mathrm{K}^{+}$ATPase inhibitors, alteration of contraction frequency might reflect their potential effects better than beating cessation (Himmel, 2013; Ikeuchi et al., 2015). However, scoring contraction frequency instead of beating arrest as endpoint studied will clearly make the assay labour intensive, thereby removing one of its advantages as a simple and cheap assay. Considering the sensitivity and practical characteriscs, both in vitro models can be used for screening cardiotoxicity. The mESC-CM beating arrest assay could be used as a first step in a tiered approach as a first screen for cardiotoxicity. Negative responding chemicals can be further evaluated in the hiPSC-CM MEA assay as a second tier to exclude cardiotoxicity for humans. Furthermore, positive chemicals in the mESC-CM beating arrest assay, can be further tested in the hiPSC-CM MEA assay to provide relevant human mechanistic data.

Finally, the hiPSC-CM MEA assay showed a high sensitivity to the effects of the chemicals. Therefore, the obtained effective concentrations were compared to internal effect concentrations related to human clinical ECG data. Given that mice show differences in the response doses, duration and certain morphology features of action potentials compared to humans (Danik et al., 2002; Edvardsson et al., 1984; Huang, 2016; Kaese and Verheule, 2012), such a direct comparison was not made for the murine data. Both the in vitro FPDc 10 and $\mathrm{AMP}_{10}$ values derived from the hiPSC-CM MEA assay matched well with the corresponding unbound $\mathrm{hECG}_{10}$ values derived from human ECG data. Remarkably, the $\mathrm{hECG}_{10}$ of amiodarone was five orders of magnitude lower than the in vitro FPDc $\mathrm{c}_{10}$. The extent to what this discrepancy is related to its high lipophilicity that results in high levels of protein binding in different matrices with $f_{u}$ values amounting to values as low as 0.0002 (Redfern et al., 2003; Ando et al., 2017), as well as other reasons underlying the discrepancy between the in vitro and in vivo situation for amiodarone remain open for further studies. Combining the in vitro cardiotoxicity data with so-called physiologically based kinetic modelling will facilitate incorporation of such factors in making ultimate QIVIVE based predictions.

The present study evaluated the sensitivity of mouse (mESC-CMs) and human (hiPSC-CMs) stem cell-derived in vitro models to screen for the potential cardiotoxicity of chemicals. The hiPSC-CM MEA assay showed a higher sensitivity for (h)ERG channel potassium and sodium channel blockers and $\mathrm{Na}^{+} / \mathrm{K}^{+}$ATPase inhibitors while the mESC-CM beating arrest assay appeared to be not responsive to (h)ERG potassium channel blockers and the $\mathrm{Na}^{+} / \mathrm{K}^{+}$ATPase inhibitor digoxin. The two models showed comparable sensitivity to calcium channel blockers 
and a $\beta$-adrenergic receptor agonist. Comparison of in vitro responses with available human clinical data revealed that effect concentrations obtained in the hiPSC-CM MEA assay were highly concordant with reported human in vivo effective concentrations of potassium and sodium channel blockers. In conclusion, both in vitro models can be considered as functional models to detect cardiotoxicity with different applicability domains. Given its ease of handling the $\mathrm{mESC}-\mathrm{CM}$ beating arrest assay may be used as a first step in a tiered approach to screen the cardiotoxicity. While negative compounds could be further tested in the hiPSC-CM MEA assay as a second tier to quantify the cardiotoxicity of compounds and reflect human in vivo cardiotoxicity.

\section{Conflict of interest}

All authors declare that they have no conflict of interest.

\section{Acknowledgements}

Work performed by Miaoying Shi was supported a Grant from the China Scholarship Council (No. 201607720029).

Part of the work was supported by a grant of the Willy van Heumenfonds.

\section{Supplementary data}

Supplementary data to this article can be found online at:

https://doi.org/10.1016/j.tiv.2020.104891 


\section{References}

Abassi, Y. A., Xi, B., Li, N. et al. (2012). Dynamic monitoring of beating periodicity of stem cell-derived cardiomyocytes as a predictive tool for preclinical safety assessment. British Journal of Pharmacology 165, 1424-1441. doi: 10.1111/j.1476-5381.2011.01623.x

Ainerua, M. O., Tinwell, J., Kompella, S. N. et al. (2020). Understanding the cardiac toxicity of the anthropogenic pollutant phenanthrene on the freshwater indicator species, the brown trout (Salmo trutta): From whole heart to cardiomyocytes. Chemosphere, 239, 124608. doi: 10.1016/j.chemosphere.2019.124608

Allen, M. J., Nichols, D. J. and Oliver, S. D. (2000). The pharmacokinetics and pharmacodynamics of oral dofetilide after twice daily and three times daily dosing. British Journal of Clinical Pharmacology 50, 247-253. doi: 10.1046/j.1365-2125.2000.00243.x

Ando, H., Yoshinaga, T., Yamamoto, W. et al. (2017). A new paradigm for drug-induced torsadogenic risk assessment using human iPS cell-derived cardiomyocytes. Journal of Pharmacological and Toxicological Methods 84, 111-127. doi: 10.1016/j.vascn.2016.12.003

Baggoi, J. and Davis, L. (1973). Plasma protein binding of digitoxin and digoxin in several mammalian species. Research in Veterinary Science 15, 81-87. doi: 10.1016/S0034-5288(18)33857-8

Bailer, A. J., Wheeler, M., Dankovic, D. et al. (2005). Incorporating uncertainty and variability in the assessment of occupational hazards. International Journal of Risk Assessment and Management 5, 344-357. doi: 10.1504/IJRAM.2005.007176

Baruscotti, M., Barbuti, A. and Bucchi, A. (2010). The cardiac pacemaker current. Journal of Molecular and Cellular Cardiology 48, 55-64. doi: 10.1016/j.yjmcc.2009.06.019

Beilmann, M., Boonen, H., Czich, A. et al. (2018). Optimizing drug discovery by investigative toxicology: Current and future trends. ALTEX-Alternatives to animal experimentation,36(2), 289-313. doi: $\underline{10.14573 / \text { altex. } 1808181}$

Bernauer, U., Oberemm, A., Madle, S. et al. (2005). The use of in vitro data in risk assessment. Basic \& clinical pharmacology \& toxicology 96(3), 176-181. doi: 10.1111/j.1742-7843.2005.pto960306.x

Bers, D. M. and Perez-Reyes, E. (1999). Ca channels in cardiac myocytes: Structure and function in Ca influx and intracellular Ca release. Cardiovascular Research 42, 339-360. doi: 10.1016/S0008-6363(99)000383

Bers, D. M. (2002). Cardiac excitation-contraction coupling. Nature 415, 198. doi: 10.1038/415198a

Buckland, S. T., Burnham, K. P. and Augustin, N. H. (1997). Model selection: An integral part of inference. Biometrics 53, 603-618. doi: $10.2307 / 2533961$

Chang, Y. X. and Mummery, C. L. (2018). Human-induced pluripotent stem cell-derived cardiomyocytes in the evaluation of cardiotoxic potential of drugs. In (eds.), Stem Cell Genetics for Biomedical Research (173194). Springer. doi: $\underline{10.1007 / 978-3-319-90695-9 \_8}$

Clements, M. and Thomas, N. (2014). High-throughput multi-parameter profiling of electrophysiological drug effects in human embryonic stem cell derived cardiomyocytes using multi-electrode arrays. Toxicological Sciences 140, 445-461. doi: 10.1093/toxsci/kfu084

Danik, S., Cabo, C., Chiello, C. et al. (2002). Correlation of repolarization of ventricular monophasic action potential with ECG in the murine heart. American Journal of Physiology-Heart and Circulatory Physiology, 283(1), H372-H381. doi: 10.1152/ajpheart.01091.2001 
Debbas, N., Bexton, R., DUCAILAR, C. et al. (1983). Relation between myocardial amiodarone concentration and QT interval. British Heart Journal, 49, 297-297. doi: 10.1093/toxsci/kfu084

DeMarco, K. and Clancy, C. E. (2016). Cardiac Na channels: Structure to function. In (eds.), Current Topics in Membranes (287-311). Elsevier. doi: 10.1016/bs.ctm.2016.05.001

Démolis, J. L., Kubitza, D., Tennezé, L. et al. (2000). Effect of a single oral dose of moxifloxacin (400 mg and $800 \mathrm{mg}$ ) on ventricular repolarization in healthy subjects. Clinical Pharmacology \& Therapeutics 68, 658-666. doi: 10.1067/mcp.2000.111482

Denning, C. and Anderson, D. (2008). Cardiomyocytes from human embryonic stem cells as predictors of cardiotoxicity. Drug Discovery Today: Therapeutic Strategies 5, 223-232. doi: 10.1016/j.ddstr.2008.08.002

Edvardsson, N., Hirsch, I., and Olsson, S. B. (1984). Right ventricular monophasic action potentials in healthy young men. Pacing and Clinical Electrophysiology, 7(5), 813-821. doi: 10.1111/j.15408159.1984.tb05622.x

Freund, C. and Mummery, C. L. (2009). Prospects for pluripotent stem cell-derived cardiomyocytes in cardiac cell therapy and as disease models. Journal of Cellular Biochemistry 107, 592-599. doi: $\underline{10.1002 / j \mathrm{jcb} .22164}$

Guo, L., Coyle, L., Abrams, R. M. et al. (2013). Refining the human iPSC-cardiomyocyte arrhythmic risk assessment model. Toxicological Sciences 136, 581-594. doi: $\underline{10.1093 / \text { toxsci/kft205 }}$

Halbach, M., Egert, U., Hescheler, J. et al. (2003). Estimation of action potential changes from field potential recordings in multicellular mouse cardiac myocyte cultures. Cellular Physiology and Biochemistry 13, 271-284. doi: $10.1159 / 000074542$

Harris, K., Aylott, M., Cui, Y. et al. (2013). Comparison of electrophysiological data from human-induced pluripotent stem cell-derived cardiomyocytes to functional preclinical safety assays. Toxicological Sciences 134, 412-426. doi: $10.1093 /$ toxsci/kft113

Heath, B., Cui, Y., Worton, S. et al. (2011). Translation of flecainide-and mexiletine-induced cardiac sodium channel inhibition and ventricular conduction slowing from nonclinical models to clinical. Journal of Pharmacological and Toxicological Methods 63, 258-268. doi: 10.1016/j.vascn.2010.12.004

Himmel, H. M. (2013). Drug-induced functional cardiotoxicity screening in stem cell-derived human and mouse cardiomyocytes: Effects of reference compounds. Journal of Pharmacological and Toxicological Methods 68, 97-111. doi: 10.1016/j.vascn.2013.05.005

Hinderling, P., Dilea, C., Koziol, T. et al. (1993). Comparative kinetics of sematilide in four species. Drug Metabolism and Disposition 21, 662-669. Available at: http://dmd.aspetjournals.org/content/21/4/662.long [Accessed August 8, 2019]

Huang, C. L.-H. (2016). Murine electrophysiological models of cardiac arrhythmogenesis. Physiological Reviews 97, 283-409. doi: $10.1152 /$ physrev.00007.2016

Ikeuchi, T., Espulgar, W., Shimizu, E. et al. (2015). Optical microscopy imaging for the diagnosis of the pharmacological reaction of mouse embryonic stem cell-derived cardiomyocytes (mESC-CMs). Analyst 140, 6500-6507. doi: 10.1039/C5AN01144B

Jeevaratnam, K., Chadda, K. R., Huang, C. L.-H. et al. (2018). Cardiac potassium channels: Physiological insights for targeted therapy. Journal of Cardiovascular Pharmacology and Therapeutics 23, 119-129. doi: $\underline{10.1177 / 1074248417729880}$ 
Jonsson, M. K., Wang, Q.-D. and Becker, B. (2011). Impedance-based detection of beating rhythm and proarrhythmic effects of compounds on stem cell-derived cardiomyocytes. Assay and Drug Development Technologies 9, 589-599. doi: 10.1089/adt.2011.0396

Kaese, S., and Verheule, S. (2012). Cardiac electrophysiology in mice: a matter of size. Frontiers in physiology, 3, 345. doi: 10.3389/fphys.2012.00345

Kamelia, L., Louisse, J., de Haan, L. et al. (2017). Prenatal developmental toxicity testing of petroleum substances: Application of the mouse embryonic stem cell test (EST) to compare in vitro potencies with potencies observed in vivo. Toxicology in Vitro 44, 303-312. doi: 10.1016/j.tiv.2017.07.018

Karakikes, I., Ameen, M., Termglinchan, V. et al. (2015). Human induced pluripotent stem cell-derived cardiomyocytes: insights into molecular, cellular, and functional phenotypes. Circulation research, 117(1), 80-88.doi: 10.1161/CIRCRESAHA.117.305365

Kelly, J. and McDevitt, D. (1978). Plasma protein binding of propranolol and isoprenaline in hyperthyroidism and hypothyroidism. British Journal of Clinical Pharmacology 6, 123-127. doi: 10.1111/j.13652125.1978.tb00836.x

Kettenhofen, R. and Bohlen, H. (2008). Preclinical assessment of cardiac toxicity. Drug Discovery Today 13, 702707. doi: $10.1016 /$ j.drudis.2008.06.011

Kitaguchi, T., Moriyama, Y., Taniguchi, T. et al. (2016). CSAHi study: Evaluation of multi-electrode array in combination with human ips cell-derived cardiomyocytes to predict drug-induced QT prolongation and arrhythmia - effects of 7 reference compounds at 10 facilities. Journal of Pharmacological and Toxicological Methods 78, 93-102. doi: 10.1016/j.vascn.2015.12.002

Kitaguchi, T., Moriyama, Y., Taniguchi, T. et al. (2017). CSAHi study: Detection of drug-induced ion channel/receptor responses, QT prolongation, and arrhythmia using multi-electrode arrays in combination with human induced pluripotent stem cell-derived cardiomyocytes. Journal of Pharmacological and Toxicological Methods 85, 73-81. doi: 10.1016/j.vascn.2017.02.001

Kola, I. and Landis, J. (2004). Can the pharmaceutical industry reduce attrition rates? Nature Reviews Drug Discovery 3, 711. doi: $10.1038 / \mathrm{nrd} 1470$

Kolossov, E., Lu, Z., Drobinskaya, I. et al. (2005). Identification and characterization of embryonic stem cellderived pacemaker and atrial cardiomyocytes. The FASEB journal 19, 577-579. doi: 10.1096/fj.03$\underline{1451 \mathrm{fje}}$

Kramer, J., Obejero-Paz, C. A., Myatt, G. et al. (2013). Mice models: Superior to the hERG model in predicting torsade de pointes. Scientific Reports 3, 2100. doi: $10.1038 /$ srep02100

Kramer, P., Horenkamp, J., Quellhorst, E. et al. (1970). Comparative studies on dialysance and renal clearance of various cardiac glycosides. Proc. Europ. Dial. Transpl. Ass, Pitman \& Medical 7, 212-216. Available at: https://link.springer.com/article/10.1007/BF01488780 [Accessed August 8, 2019]

Kratz, J.M., Grienke, U., Scheel, O. et al. (2017). Natural products modulating the hERG channel: heartaches and hope. Natural product reports, 34(8), 957-980. doi: 10.1039/C7NP00014F

Laurila, E., Ahola, A., Hyttinen, J. et al. (2016). Methods for in vitro functional analysis of ipsc derived cardiomyocytes - special focus on analyzing the mechanical beating behavior. Biochimica et Biophysica Acta (BBA)-Molecular Cell Research 1863, 1864-1872. doi: 10.1016/j.bbamcr.2015.12.013

Lewandowski, J., Kolanowski, T. J. and Kurpisz, M. (2017). Techniques for the induction of human pluripotent stem cell differentiation towards cardiomyocytes. Journal of Tissue Engineering and Regenerative Medicine 11, 1658-1674. doi: 10.1002/term.2117 
Li, G.-R., Feng, J., Yue, L. et al. (1996). Evidence for two components of delayed rectifier $\mathrm{K}^{+}$current in human ventricular myocytes. Circulation Research 78, 689-696. doi: 10.1161/01.RES.78.4.689

Li, X., Zhang, R., Zhao, B. et al. (2016). Cardiotoxicity screening: A review of rapid-throughput in vitro approaches. Archives of Toxicology 90, 1803-1816. doi: 10.1007/s00204-015-1651-1

Lombardo, F., Obach, R. S., Shalaeva, M. Y. et al. (2002). Prediction of volume of distribution values in humans for neutral and basic drugs using physicochemical measurements and plasma protein binding data. Journal of Medicinal Chemistry 45, 2867-2876. doi: 10.1021/jm0200409

Louisse, J., Beekmann, K. and Rietjens, I. M. (2017). Use of physiologically based kinetic modeling-based reverse dosimetry to predict in vivo toxicity from in vitro data. Chemical Research in Toxicology 30, 114-125. doi: $10.1021 /$ acs.chemrestox.6b00302

Ma, J., Guo, L., Fiene, S. J. et al. (2011). High purity human-induced pluripotent stem cell-derived cardiomyocytes: Electrophysiological properties of action potentials and ionic currents. American Journal of PhysiologyHeart and Circulatory Physiology 301, H2006-H2017. doi: 10.1152/ajpheart.00694.2011

Maltsev, V. A., Wobus, A. M., Rohwedel, J. et al. (1994). Cardiomyocytes differentiated in vitro from embryonic stem cells developmentally express cardiac-specific genes and ionic currents. Circulation Research 75, 233-244. doi: $\underline{10.1161 / 01 . R E S .75 .2 .233}$

Nerbonne, J. M. (2004). Studying cardiac arrhythmias in the mouse- - a reasonable model for probing mechanisms? Trends in Cardiovascular Medicine 14, 83-93. doi: 10.1016/j.tcm.2003.12.006

Nicolas, J., Hendriksen, P. J., de Haan, L. H. et al. (2015). In vitro detection of cardiotoxins or neurotoxins affecting ion channels or pumps using beating cardiomyocytes as alternative for animal testing. Toxicology in Vitro 29, 281-288. doi: 10.1016/j.tiv.2014.11.010

Nozaki, Y., Honda, Y., Watanabe, H. et al. (2016). CSAHi study: Validation of multi-electrode array systems (MEA60/2100) for prediction of drug-induced proarrhythmia using human iPS cell-derived cardiomyocytes-assessment of inter-facility and cells lot-to-lot-variability. Regulatory Toxicology and Pharmacology 77, 75-86. doi: 10.1016/j.yrtph.2016.02.007

Nozaki, Y., Honda, Y., Watanabe, H. et al. (2017). CSAHi study-2: Validation of multi-electrode array systems (MEA60/2100) for prediction of drug-induced proarrhythmia using human iPS cell-derived cardiomyocytes: Assessment of reference compounds and comparison with non-clinical studies and clinical information. Regulatory Toxicology and Pharmacology 88, 238-251. doi: 10.1016/j.yrtph.2017.06.006

Pang, L., Sager, P., Yang X. et al. (2019). Workshop Report: FDA Workshop on Improving Cardiotoxicity Assessment With Human-Relevant Platforms. Circulation research, 125(9), 855-867. doi: 10.1161/CIRCRESAHA.119.315378

Pourrier, M., and Fedida, D. (2020). The Emergence of Human Induced Pluripotent Stem Cell-Derived Cardiomyocytes (hiPSC-CMs) as a Platform to Model Arrhythmogenic Diseases. International Journal of Molecular Sciences, 21(2), 657. doi: 10.3390/ijms21020657

Pouton, C. W. and Haynes, J. M. (2007). Embryonic stem cells as a source of models for drug discovery. Nature Reviews Drug Discovery 6, 605. doi: $10.1038 / \mathrm{nrd} 2194$

Priest, B. T. and McDermott, J. S. (2015). Cardiac ion channels. Channels 9, 352-359. doi: $\underline{10.1080 / 19336950.2015 .1076597}$

Redfern, W., Carlsson, L., Davis, A. et al. (2003). Relationships between preclinical cardiac electrophysiology, clinical QT interval prolongation and torsade de pointes for a broad range of drugs: Evidence for a 
provisional safety margin in drug development. Cardiovascular Research 58, 32-45. doi: $\underline{10.1016 / \mathrm{S} 0008-6363(02) 00846-5}$

Rehnelt, S., Malan, D., Juhasz, K. et al. (2017). Frequency-dependent multi-well cardiotoxicity screening enabled by optogenetic stimulation. International Journal of Molecular Sciences 18, 2634. doi: $\underline{10.3390 / \mathrm{ijms} 18122634}$

Rougier, J.-S. and Abriel, H. (2016). Cardiac voltage-gated calcium channel macromolecular complexes. Biochimica et Biophysica Acta (BBA)-Molecular Cell Research 1863, 1806-1812. doi: $\underline{10.1016 / \text { i.bbamcr.2015.12.014 }}$

Sadanaga, T., Ogawa, S., Okada, Y. et al. (1993). Clinical evaluation of the use-dependent QRS prolongation and the reverse use-dependent QT prolongation of class I and class III antiarrhythmic agents and their value in predicting efficacy. American Heart Journal 126, 114-121. doi: 10.1016/S0002-8703(07)80017-2

Sala, L., Ward-van Oostwaard, D., Tertoolen, L. G. et al. (2017). Electrophysiological analysis of human pluripotent stem cell-derived cardiomyocytes (hPSC-CMs) using multi-electrode arrays (MEAs). JoVE (Journal of Visualized Experiments) 123, e55587. doi: $10.3791 / 55587$

Sartiani, L., Cerbai, E. and Mugelli, A. (2011). The funny current in cardiac non-pacemaker cells: Functional role and pharmacological modulation. In (eds.), Modern Pacemakers-present and Future (595-610). IntechOpen. doi: $10.5772 / 13086$

Schwinger, R. H., Bundgaard, H., Müller-Ehmsen, J. et al. (2003). The Na, K-ATPase in the failing human heart. Cardiovascular rResearch 57, 913-920. doi: 10.1016/S0008-6363(02)00767-8

Seiler, A. E. and Spielmann, H. (2011). The validated embryonic stem cell test to predict embryotoxicity in vitro. Nature Protocols 6, 961-978. doi: 10.1038/nprot.2011.348

Shimizu, W., Antzelevitch, C., Suyama, K. et al. (2000). Effect of sodium channel blockers on st segment, QRS duration, and corrected QT interval in patients with brugada syndrome. Journal of Cardiovascular Electrophysiology 11, 1320-1329. doi: 10.1046/j.1540-8167.2000.01320.x

Stevens, J. L. and Baker, T. K. (2009). The future of drug safety testing: Expanding the view and narrowing the focus. Drug Discovery Today 14, 162-167. doi: 10.1016/j.drudis.2008.11.009

Tamargo, J., Caballero, R., Gómez, R. et al. (2004). Pharmacology of cardiac potassium channels. Cardiovascular Research 62, 9-33. doi: 10.1016/j.cardiores.2003.12.026

Tennezé, L., Tarral, E., Ducloux, N. et al. (2002). Pharmacokinetics and electrocardiographic effects of a new controlled-release form of flecainide acetate: Comparison with the standard form and influence of the CYP2D6 polymorphism. Clinical Pharmacology \& Therapeutics 72, 112-122. Doi: $\underline{10.1067 / \mathrm{mcp} .2002 .125946}$

Tertoolen, L., Braam, S., van Meer, B. et al. (2018). Interpretation of field potentials measured on a multi electrode array in pharmacological toxicity screening on primary and human pluripotent stem cell-derived cardiomyocytes. Biochemical and Biophysical Research Communications 497, 1135-1141. doi: $\underline{10.1016 / \mathrm{j} . \mathrm{bbrc} .2017 .01 .151}$

Valentin, J. P. (2010). Reducing QT liability and proarrhythmic risk in drug discovery and development. British Journal of Pharmacology 159, 5-11. doi: 10.1111/j.1476-5381.2009.00547.x

Vandenberk, B., Vandael, E., Robyns, T. et al. (2016). Which QT correction formulae to use for QT monitoring? Journal of the American Heart Association, 5(6), e003264. doi: $\underline{10.1161 / J A H A .116 .003264 ~}$ 
Wheeler, M. W. and Bailer, A. J. (2007). Properties of model-averaged bmdls: A study of model averaging in dichotomous response risk estimation. Risk Analysis: An International Journal 27, 659-670. doi: $\underline{10.1111 / \mathrm{j} .1539-6924.2007 .00920 . \mathrm{x}}$

Wobus, A. M., Wallukat, G. and Hescheler, J. (1991). Pluripotent mouse embryonic stem cells are able to differentiate into cardiomyocytes expressing chronotropic responses to adrenergic and cholinergic agents and $\mathrm{Ca}^{2+}$ channel blockers. Differentiation 48, 173-182. doi: 10.1111/j.1432-0436.1991.tb00255.x

$\mathrm{Xu}, \mathrm{H}$., Guo, W. and Nerbonne, J. M. (1999). Four kinetically distinct depolarization-activated $\mathrm{K}^{+}$currents in adult mouse ventricular myocytes. The Journal of General Physiology 113, 661-678. doi: $\underline{10.1085 / \text { jgp.113.5.661 }}$

Zhou, J., Kodirov, S., Murata, M. et al. (2003). Regional upregulation of Kv2. 1-encoded current, $I_{\mathrm{k}}$, slow2, in $\mathrm{Kv} 1 \mathrm{DN}$ mice is abolished by crossbreeding with Kv2DN mice. American Journal of Physiology-Heart and Circulatory Physiology 284, H491-H500. doi: 10.1152/ajpheart.00576.2002

Zwartsen, A., de Korte, T., Nacken, P. et al. (2019). Cardiotoxicity screening of illicit drugs and new psychoactive substances (NPS) in human iPSC-derived cardiomyocytes using microelectrode array (MEA) recordings. Journal of molecular and cellular cardiology, 136, 102-112.doi: $\underline{10.1016 / j . y j m c c .2019 .09 .007}$ 


$$
3
$$




\section{Chapter 3}

\section{Integrating in vitro data and}

physiologically based kinetic modelling-facilitated reverse dosimetry to predict human cardiotoxicity of methadone

Miaoying Shi, Hans Bouwmeester, Ivonne M. C. M. Rietjens, Marije Strikwold

Published in Archives of Toxicology (2020), 94(8), 2809-2827.

https://doi.org/10.1007/s00204-020-02766-7 


\section{Abstract}

Development of novel testing strategies to detect adverse human health effects is of interest to replace in vivo based drug and chemical safety testing. The aim of the present study was to investigate whether physiologically based kinetic (PBK) modelling-facilitated conversion of in vitro toxicity data is an adequate approach to predict in vivo cardiotoxicity in humans. In order to enable evaluation of predictions made, methadone was selected as the model compound, being a compound for which data on both kinetics and cardiotoxicity in humans are available. A PBK model for methadone in humans was developed and evaluated against available kinetic data presenting an adequate match. Use of the developed PBK model to convert concentration-response curves for the effect of methadone on human induced pluripotent stem cell-derived cardiomyocytes (hiPSC-CM) in the so-called multi-electrode array (MEA) assay, resulted in predictions for in vivo dose-response curves for methadone induced cardiotoxicity that matched the available in vivo data. The results also revealed differences in protein plasma binding of methadone to be a potential factor underlying variation between individuals with respect to sensitivity towards the cardiotoxic effects of methadone. The present study provides a proof-of-principle of using PBK modelling-based reverse dosimetry of in vitro data for the prediction of cardiotoxicity in humans, providing a novel testing strategy in cardiac safety studies. 


\section{Introduction}

Traditional approaches for the risk and safety assessment of compounds rely heavily on toxicity data derived from laboratory animals, which are gradually being recognized as inappropriate models for the prediction of human health effects due to toxicodynamic and toxicokinetic differences between animals and human (Ewart et al. 2014; Pang et al. 2019). This consideration as well as the fact that animal-based testing strategies are cost and labour intensive, while also increasingly considered unethical, has stimulated the development of novel testing strategies, leading to a paradigm shift in toxicity testing (Judson et al. 2014). Novel testing strategies generally apply in vitro assays and take into account insight in the modes of action underlying the toxicity (Bernauer et al. 2005). However, in vitro assays provide hazard information and concentration-response curves that require translation to corresponding human dose-response curves, taking into account human toxicokinetics, to enable their use in human risk and safety assessment of compounds (Bell et al. 2018; Blaauboer 2010).

Over the last decade several proof-of-principle studies indicated that combining in vitro toxicity assays with physiologically based kinetic (PBK) modelling, which describes the absorption, distribution, metabolism and excretion (ADME) of a compound in a defined species, can adequately predict in vivo dose-response curves (Louisse et al. 2017; Rietjens et al. 2011). For example, quantitative in vitro to in vivo extrapolation (QIVIVE) using PBK modelling-based reverse dosimetry was shown to adequately predict the in vivo toxicity for different endpoints, including developmental toxicity (Li et al. 2017; Louisse et al. 2010; Strikwold et al. 2013, 2017), liver toxicity (Ning et al. 2017), nephrotoxicity (Abdullah et al. 2016) and neurotoxicity (Zhao et al. 2019). To further explore the potential applicability of this in vitro-in silico approach, the aim of the present study was to investigate whether the PBK modelling-based reverse dosimetry can be extended to predict in vivo cardiotoxicity in human thereby providing a novel testing strategy for cardiac safety testing.

Cardiotoxicity is an important endpoint in pharmaceutical safety testing and has been a leading cause of drug attrition in preclinical drug development (Pang et al. 2019; Stevens and Baker 2009). In addition, cardiotoxicity is also a relevant endpoint in food safety, given that many food-borne alkaloids from botanicals and botanical preparations, including for example synephrine from bitter orange (Citrus aurantium) and nuciferine from lotus (Nelumbo nucifera), raise a concern with respect to potential cardiotoxicity (Kratz et al. 2017). Potential 
cardiotoxicity includes functional and structural disruption of the cardiovascular system by interfering with ion channels, intracellular organelles and cellular signalling pathways (Clements et al. 2015; Pang et al. 2019). Particularly cardiac electrophysiological alterations such as delayed ventricular repolarization are endpoints of interest for cardiac safety assessment. Delayed ventricular repolarization can result in a prolonged QTc interval (time from ventricular depolarization and repolarization corrected for heart rate) in the electrocardiogram (ECG) which is associated with increased risk of arrhythmia including polymorphic ventricular tachyarrhythmia (torsade de pointes, TdP) (Ewart et al. 2012; Harris et al. 2013; Kannankeril et al. 2010; Redfern et al. 2003; Wakefield et al. 2002). Current regulatory guidelines to evaluate in vitro electrophysiological cardiotoxicity are based on ion channel inhibition assays using cell lines transfected with specific ion channels, including especially human ether-à-go-go-related gene (hERG) channels which play a critical role in cardiac repolarization (ICH 2005a; Martin et al. 2004; Zwartsen et al. 2019). However, such an approach focussing on a single type of ion channel fails to address effects induced on other channels (Mirams et al. 2011; Rehnelt et al. 2017). Recently, human induced pluripotent stem cell-derived cardiomyocytes (hiPSC-CMs) have been reported to provide a physiological relevant in vitro model for human cardiotoxicity testing. These hiPSC-CMs express major cardiac ion channels and show typical electrophysiological responses upon the exposure to compounds (Garg et al. 2018; Ma et al. 2011). In the present study hiPSC-CMs were applied in combination with the multi-electrode array (MEA) technique measuring the extracellular field potential of electrically active cardiomyocytes, which is considered a promising tool to assess electrophysiological alteration and arrhythmias (Ando et al. 2017; Harris et al. 2013; Kitaguchi et al. 2017; Li et al. 2016). The parameters obtained from extracellular field potential waveforms are considered to resemble the parameters observed in the human ECG (Zwartsen et al. 2019), which allows use of the hiPSC-CM MEA assay as an adequate in vitro model for QIVIVE.

The model compound selected for the present study was methadone (Fig. 1). Methadone is a synthetic drug for the treatment of opioid dependence and chronic pain. Methadone is metabolized by cytochromes P450 (CYP) mainly in the liver (Eap et al. 2002; Nilsson et al. 1982). The primary metabolite, 2-ethylidene-1,5-dimethyl-3,3-diphenylpyrrolidine (EDDP), is formed via $\mathrm{N}$-demethylation and cyclisation, and a subsequent $\mathrm{N}$-demethylation leads to the secondary metabolite, 2-ethyl-5-methyl-3,3-diphenylpyrroline (EMDP) (Fig. 1). Methadone has been reported to cause cardiotoxic side effects in human clinical studies in which prolonged 
QTc interval and TdP have been observed in subjects receiving methadone maintenance treatment (Alinejad et al. 2015; Eap et al. 2002; Justo et al. 2006). Several in vitro studies using electrophysiological-based patch clamp demonstrated an association between the cardiotoxicity of methadone and the inhibition of hERG channels (Eap et al. 2007; Kuryshev et al. 2010).

In the present study, the in vitro concentration-dependent cardiotoxicity of methadone and its metabolites EDDP and EMDP was quantified in hiPSC-CM using the MEA technique. Additionally, a PBK model for methadone kinetics in human was developed by integrating data from literature as well as experimentally obtained metabolic parameters. This PBK model was subsequently used to translate the in vitro toxicity data to predict in vivo cardiotoxicity in human. The data thus obtained were compared to available data on the effect of methadone on cardiac parameters in subjects that received methadone maintenance treatment.

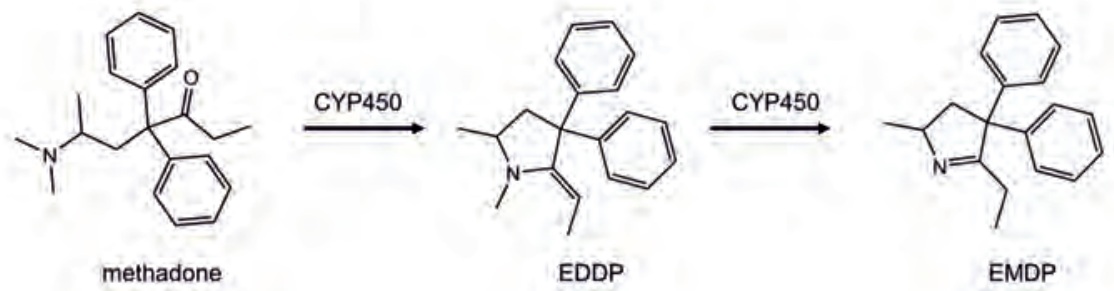

Fig. 1 Metabolic conversion of methadone to 2-ethylidene-1,5-dimethyl-3,3-diphenylpyrrolidine (EDDP), and 2-ethyl-5-methyl-3,3-diphenylpyrroline (EMDP) by cytochromes P450 (CYP450)

\section{Materials and methods}

\subsection{Chemical and biological materials}

Methadone hydrochloride ( $\geq 98 \%$ ), EDDP perchlorate ( $\geq 98 \%$ ), EMDP hydrochloride solution $(1.0 \mathrm{mg} / \mathrm{ml})$, Tris (hydroxymethyl) aminomethane (Trizma ${ }^{\circledR}$ base), ammonium formate and fibronectin were purchased from Sigma-Aldrich (Zwijndrecht, The Netherlands). Methadone and metabolites were ordered under the opium exemption license number $10478303 \mathrm{WCO}$, which is registered at Farmatec (executive organization of the Ministry of Health, Welfare and Sport, The Hague, The Netherlands). Dimethyl sulfoxide (DMSO, 99.7\%) was obtained from Merck (Schiphol-Rijk, The Netherlands). Phosphate buffered saline (PBS) was purchased from Gibco (Paisley, Scotland, UK). Acetonitrile (UPLC/MS grade) was obtained from Biosolve BV (Valkenswaard, The Netherlands). hiPSC-CM (Pluricyte ${ }^{\circledR}$ Cardiomyocytes, cat\# PCMI- 
1031-1, lot\# 60151) and Pluricyte ${ }^{\circledR}$ Cardiomyocyte Medium were obtained from Ncardia (Leiden, The Netherlands). Pooled human liver microsomes (from 150 donors), pooled human intestinal microsomes (from 7 donors) and reduced nicotinamide adenine dinucleotide phosphate (NADPH) regenerating system solution A and solution B were purchased from Corning (Woburn, MA, USA). Pooled human plasma and rapid equilibrium dialysis (RED) materials, including RED inserts, RED base plates and sealing tape were obtained from Thermo Fisher Scientific (Bleiswijk, The Netherlands).

\subsection{General outline of the PBK modelling-based reverse dosimetry approach}

The PBK modelling-based reverse dosimetry approach to predict the in vivo dose-response curves from in vitro cardiotoxicity concentration-response data included the following steps: (1) establishment of the in vitro concentration-response curves for methadone and its metabolites EDDP and EMDP in hiPSC-CM using the MEA, (2) development of a PBK model for methadone and its metabolites in human using metabolic parameters obtained from in vitro incubations with pooled human liver microsomes, and parameters derived from in silico simulations and the literature, (3) evaluation of the PBK model, (4) translation of in vitro concentration-response curves to the in vivo dose-response curves using the PBK model, and (5) evaluation of the PBK modelling-based reverse dosimetry approach by comparing predicted dose-response data to data obtained from literature on the effect of methadone on cardiac parameters in subjects receiving methadone maintenance treatment.

\subsection{In vitro cardiotoxicity of methadone and metabolites in hiPSC-CM using the MEA}

The MEA system of Multi Channel System (MCS GmbH, Ruetlingen, Germany) combined with Pluricyte ${ }^{\circledR}$ Cardiomyocytes was used to detect the cardiotoxicity of methadone, and the metabolites EDDP and EMDP. The Pluricyte ${ }^{\circledR}$ Cardiomyocytes were thawed and seeded on the 6-well MEA chips (60-6well MEA200/30iR-Ti-tcr, MCS GmbH) according to the manufacturer's protocol. Briefly, each well of the MEA chips was precoated with $50 \mu \mathrm{g} / \mathrm{ml}$ fibronectin for 3 hours in the incubator at $37^{\circ} \mathrm{C}$ with $5 \% \mathrm{CO}_{2}$. The fibronectin coating solution was aspirated before seeding. Cells were thawed in the incubator at $37{ }^{\circ} \mathrm{C}$ for exactly $4 \mathrm{~min}$ and carefully transferred to a $50 \mathrm{ml}$ tube. The original vial was rinsed with serum free Pluricyte $^{\circledR}$ Cardiomyocyte Medium and added drop-wise to the tube containing the cardiomyocytes. Subsequently cell counting was manually performed by using $20 \mu \mathrm{l}$ of 
obtained homogenous cell suspension in a Buerker-Tuerk Counting Chamber (Marienfeld Superior GmbH \& Co. KG, Lauda-Königshofen, Germany) and at the same time the remaining cells were centrifuged at $300 \mathrm{~g}$ for $3 \mathrm{~min}$. Then the supernatant was removed and medium was drop-wisely added to reach the aimed concentration of cells in the suspension $\left(10^{4}\right.$ cells $\left./ \mu 1\right) .2$ $\mu \mathrm{l}$ cell suspension per well was placed on the 6-well MEA chips in a density of $10^{4}$ cells $/ \mu 1$. After 3 hours incubation $\left(37^{\circ} \mathrm{C}, 5 \% \mathrm{CO}_{2}\right), 200 \mu \mathrm{l}$ of medium was filled into each well of the MEA chips which were subsequently incubated at $37{ }^{\circ} \mathrm{C}$ with $5 \% \mathrm{CO}_{2}$ and refreshed with medium every 2 days.

At 7-8 days after seeding, MEA chips were placed on the headstage of a MEA2100-system $(\mathrm{MCS} \mathrm{GmbH})$ integrated with the chamber providing a stable atmosphere $\left(37^{\circ} \mathrm{C}, 5 \% \mathrm{CO}_{2}\right)$ to record the extracellular field potential (Fig. 2) of spontaneous beating hiPSC-CM. After an equilibration time of $20 \mathrm{~min}$, half of the medium $(100 \mu \mathrm{l})$ in each well was replaced by culture medium containing $0.2 \%(\mathrm{v} / \mathrm{v})$ DMSO to reach a final concentration of $0.1 \%(\mathrm{v} / \mathrm{v}) \mathrm{DMSO}$, which was used as baseline condition. Subsequently the model compounds were tested in separate wells, and each test compound was cumulatively added to the well with increasing concentrations in the same way (Harris et al., 2013; Nozaki et al., 2017; Ando et al., 2017). At each concentration, the extracellular field potential was recorded for $1 \mathrm{~min}$ after $10 \mathrm{~min}$ exposure. Stock solutions of model compounds were prepared in DMSO and further diluted in Pluricyte ${ }^{\circledR}$ Cardiomyocyte Medium to make exposure medium with the final concentration of $0.1 \%(\mathrm{v} / \mathrm{v})$ DMSO. The following concentrations were tested, 0.01, 0.03, 0.1, 0.2, 0.3, 0.4, 1, 3, 10, $30 \mu \mathrm{M}$ (methadone), 0.01, 0.03, 0.1, 0.3, 1, 3, 10, $30 \mu \mathrm{M}$ (EDDP) and 0.1, 0.3, 1, 3, 10, $30 \mu \mathrm{M}$ (EMDP), at which no cytotoxicity was observed (data not shown). The test concentrations of methadone were based on reported human methadone plasma concentrations that were observed after oral methadone treatment. Same test concentrations were chosen for EDDP and EMDP, which enables definition of concentration-dependent curves for EDDP and EMDP that allow potency comparison.

One well of $0.1 \%(\mathrm{v} / \mathrm{v})$ DMSO on each MEA chip was used as the vehicle control well and run at the same time as the compound exposure wells to correct for time- and DMSO-dependent effects on the field potential. A detailed exposure scheme can be found in Fig. S1. Data were collected using Cardio 2D software (MCS GmbH) with a sample frequency of $10 \mathrm{kHz}$ and a $0.1-3.5 \mathrm{kHz}$ band-pass filter. 


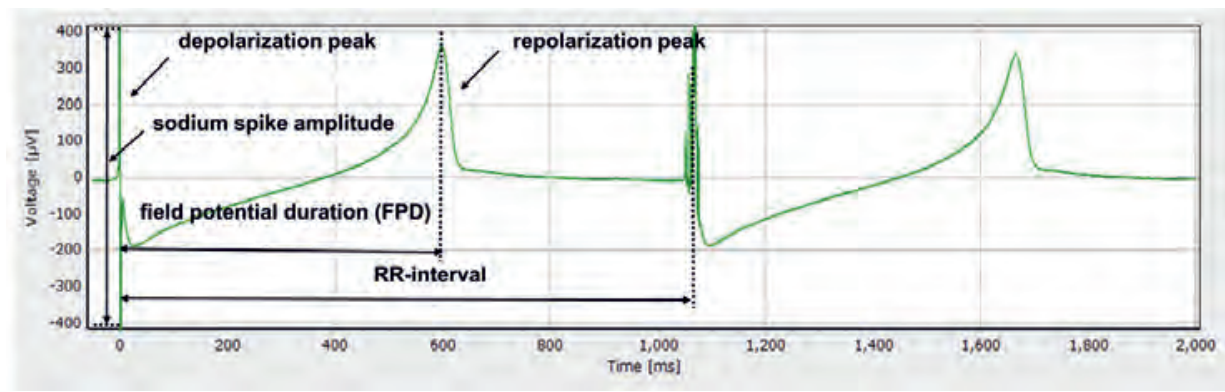

Fig. 2 Typical extracellular field potential waveform consisting of a rapid upstroke corresponding to depolarization, a slow wave/plateau and a repolarization peak. Signals were generated under the baseline condition $(0.1 \%(\mathrm{v} / \mathrm{v}) \mathrm{DMSO})$ in Pluricyte cardiomyocytes cultured in 6-well MEA-chips measured by the MEA2100-System platform of MCS

After exposure, MEA data generated from the electrodes showing stable baseline field potential with clearly visible depolarization (peak amplitude $\geq 200 \mu \mathrm{V}$ ) and repolarization peaks (peak amplitude $\geq 20 \mu \mathrm{V}$ ) (Ando et al. 2017; Sala et al. 2017) were selected for further analysis using Multiwell-Analyzer software Version 1.5.1.0 (MCS GmbH). Field potential duration (FPD) was defined as duration between the beginning of the sodium spike and the repolarizing peak (Fig. 2). RR-intervals were defined as the duration between two depolarization peaks (Fig. 2). The FPD and RR-interval were measured as the average of at least 30 beats from one-min recording at each concentration of the test compound. In addition, the Fridericia formula (equation 1) was applied to correct for the effect of beat rate on FPD (Vandenberk et al. 2016) as widely used in other MEA studies (Ando et al. 2017; Kitaguchi et al. 2017):

$$
\mathrm{FPDc}=\frac{\mathrm{FPD}}{\sqrt[3]{\mathrm{RR} \text { interval }}}
$$

In this formula the FPD and RR-interval are expressed in seconds. Data were collected from at least three independent experiments (4-8 wells, 26-38 electrodes), using a new vial of cells (all from the same batch) at each independent experiment. In vitro cardiotoxic effects are expressed as relative percentage of FPDc compared to the FPDc results obtained for the baseline condition $(0.1 \%(\mathrm{v} / \mathrm{v}) \mathrm{DMSO})$ and further corrected for the time- and DMSOdependent effects by subtracting the response of $0.1 \%(\mathrm{v} / \mathrm{v})$ DMSO obtained from the corresponding time-matched vehicle control well. The concentrations inducing irregularities in the field potential trace (Fig. S2) were also noted. Such irregularities included arrhythmia-type changes in the waveform, a flattened unclear second peak and/or beating arrest (Asakura et al. 
2015; Kitaguchi et al. 2017; Nakamura et al. 2014; Zwartsen et al. 2019). Concentrations inducing these irregularities were excluded from the FPD analysis since the FPD and RRinterval could not be determined.

Effective concentrations expressed as the FPDc (\% to the baseline control) were determined by using the benchmark dose (BMD) approach which was performed as described in the section on "Evaluation of the PBK modelling-based reverse dosimetry approach". The concentrationresponse curves were plotted with GraphPad Prism 5.0 using the four-parameters logistic fit (GraphPad Software Inc., San Diego, USA). Each data point is presented as the mean value of at least three independent experiments \pm standard deviation (SD). Statistical significance of the changes in response of cells exposed to the compound compared to the solvent control was analyzed by one-way ANOVA followed by post Dunnett test. Values of $p<0.05$ were regarded as statistically significant $\left(p<0.05: *, p<0.01\right.$ : $^{* *}$ and $\left.p<0.001: * * *\right)$. Statistical analysis was performed by GraphPad Prism 5.0 (GraphPad Software Inc.).

\subsection{In vitro microsomal incubations}

In vitro incubations were performed to obtain the kinetic parameters for the conversion of methadone by human liver microsomes. To this end, incubation conditions were optimized to obtain linear reaction rates with respect to microsomal protein levels $(0.1-2 \mathrm{mg} / \mathrm{ml}$ protein) and incubation time (1-120 $\mathrm{min}$ ) at $50 \mu \mathrm{M}$ methadone. The final incubation mixtures (final volume of $160 \mu \mathrm{l})$ consisted of $0.1 \mathrm{M}$ Tris- $\mathrm{HCl}(\mathrm{pH}$ 7.4-7.5), NADPH regeneration system (final concentrations $1.3 \mathrm{mM} \mathrm{NADP}^{+}, 3.3 \mathrm{mM}$ glucose-6-phosphate, $0.4 \mathrm{U} / \mathrm{ml}$ glucose-6-phosphate dehydrogenase and $3.3 \mathrm{mM}$ magnesium chloride) and methadone at eight final concentrations ranging from 10 to $1500 \mu \mathrm{M}$ diluted from a $100 \mathrm{mM}$ stock solution in water. The test concentrations were chosen to enable adequate analysis of Michaelis-Menten kinetics. After one-min pre-incubation of this solution at $37^{\circ} \mathrm{C}$, the reactions were initiated by addition of human liver microsomes giving a final concentration of $0.5 \mathrm{mg} / \mathrm{ml}$ microsomal protein and incubations were performed in a shaking water bath at $37^{\circ} \mathrm{C}$ for $40 \mathrm{~min}$. Control incubations were performed in the absence of NADPH which was replaced with Tris-HCl. The reactions were terminated by addition of $40 \mu 1$ ice-cold acetonitrile. Samples were kept on ice for at least $20 \mathrm{~min}$ and then centrifuged at $18,000 \mathrm{~g}$ for $5 \mathrm{~min}$ at $4{ }^{\circ} \mathrm{C}$ to precipitate microsomal proteins. The supernatant was collected for the quantification of EDDP formation, which was analyzed by Ultra Performance Liquid Chromatography PhotoDiode Array (UPLC-PDA, Waters) as described in the "Quantification of methadone and its metabolites by UPLC-PDA analysis" section. 
The formation of the secondary metabolite EMDP from EDDP was investigated by incubating $1000 \mu \mathrm{M}$ EDDP under the same conditions as described above for the microsomal methadone incubations. The kinetic parameters for the conversion of methadone by intestinal microsomes were determined under the same conditions as the incubations with liver microsomes after the incubation conditions were optimized with respect to microsomal protein levels $(0.1-2 \mathrm{mg} / \mathrm{ml}$ protein) and incubation time (1-120 $\mathrm{min})$ at $50 \mu \mathrm{M}$ methadone.

The apparent maximum velocity $\left(\mathrm{V}_{\max }\right)$ and the apparent Michaelis-Menten constant $\left(\mathrm{K}_{\mathrm{m}}\right)$ describing the conversion of methadone to EDDP were determined using the MichaelisMenten equation (2):

$$
\mathrm{V}=\frac{\mathrm{V}_{\max } \mathrm{x}[\mathrm{S}]}{\mathrm{K}_{\mathrm{m}}+[\mathrm{S}]}
$$

Where $[\mathrm{S}]$ is the substrate concentration $(\mu \mathrm{M})$ and $\mathrm{v}$ is the rate of EDDP formation (nmol/min/mg protein). $V_{\max }$ and $\mathrm{K}_{\mathrm{m}}$ were obtained by fitting the data to equation (2) in GraphPad Prism 5.0 (GraphPad Software Inc.). Data were collected from 3 independent experiments and each data point is presented as the mean value \pm SD.

\subsection{Determination of unbound fraction of methadone and EDDP in in vitro hiPSC-CM MEA assay medium and in human plasma}

The rapid equilibrium dialysis (RED) assay was performed to determine the unbound fraction $\left(f_{u}\right)$ of methadone and EDDP in in vitro medium and in pooled human plasma using the protocol adapted from the manufacturer of the RED device (Thermo Fisher Scientific, 2017). In short, methadone or EDDP were added to the in vitro medium or pooled human plasma to reach a concentration of $150 \mu \mathrm{M}$ in test sample solution and PBS was used as buffer. $300 \mu 1$ test sample solution and $500 \mu \mathrm{l}$ PBS were respectively added to the sample chamber and the buffer chamber of the RED insert, which was subsequently incubated for 5 hours at $37^{\circ} \mathrm{C}$ at $250 \mathrm{rpm}$ on an orbital shaker to reach equilibrium (van Liempd et al. 2011). Then $25 \mu 1$ of post-dialysis samples were collected from the sample chambers and transferred to test sample tubes followed by an addition of $25 \mu \mathrm{l}$ PBS. Equal volumes of post-dialysis samples collected from the buffer chamber which were then mixed with $25 \mu 1$ of in vitro medium or human plasma in the buffer sample tubes. Then, both samples were precipitated using $300 \mu$ cold acetonitrile/water (90/10 $\mathrm{v} / \mathrm{v}$ ). The samples were put on ice for $30 \mathrm{~min}$ followed by centrifugation for $30 \mathrm{~min}$ at $15,000 \mathrm{~g}$. Then, supernatants were collected for UPLC-PDA analysis. The fraction unbound was calculated with equation 3 (van Liempd et al. 2011; Waters et al. 2008): 


$$
\mathrm{f}_{\mathrm{u}}=\frac{\text { concentration in buffer chamer }}{\text { concentration in sample chamer }}
$$

The measurements were performed in triplicate in two independent experiments.

\subsection{Quantification of methadone and its metabolites by UPLC-PDA analysis}

The quantification of methadone and its metabolites was performed by UPLC-PDA analysis using a Waters Acquity UPLC H_class system (Etten-Leur, The Netherlands) equipped with a Waters Acquity BEH C18 $(1.7 \mu \mathrm{m}, 2.1$ x $50 \mathrm{~mm})$ column. For optimal separation, a gradient of $20 \mathrm{mM}$ ammonium formate $(\mathrm{pH}=5.7)$ (solvent $\mathrm{A}$ ) and acetonitrile (solvent $\mathrm{B}$ ) with a flow rate of $0.3 \mathrm{ml} / \mathrm{min}$ was applied as follows, the initial condition was 90:10 (A:B) then the gradient was increased linear to $98 \%$ B over $8 \mathrm{~min}$, then set to the initial conditions in $2 \mathrm{~min}$ and re-equilibrated for $5 \mathrm{~min}$. Retention times of methadone, EDDP and EMDP were 4.7, 4.4 and $6.3 \mathrm{~min}$, respectively. Identification of methadone and its metabolites was based on comparison of their retention time and UV spectrum to those of commercially available reference compounds. Quantification was based on comparison of the respective peak areas to the peak areas of corresponding calibration curves which were prepared using the reference compounds $\left(\mathrm{R}^{2}>0.999\right)$.

\subsection{Establishment of the PBK model for methadone and EDDP}

In the present paper a PBK model describing the ADME of methadone and its major metabolites in human was developed. Fig. 3 presents the schematic diagram of the PBK model including a submodel for the major metabolite EDDP and the compartments relevant for the ADME characteristics of methadone and EDDP. A submodel for EDDP was included to enable the prediction of internal concentrations of EDDP required to evaluate if EDDP will be formed in quantities that are relevant for cardiotoxicity. Considering that methadone is usually administered to the opioid dependent population or patients with chronic pain on a daily basis, a PBK model for repeated dosing of methadone was developed.

The absorption rate constant (ka) and fraction absorbed (Fa) are two key parameters describing the absorption of methadone. The uptake of methadone from the gastrointestinal (GI) tract was reported to follow a first-order process (Yang et al. 2006) with a mean ka value of 0.59 /h obtained from several studies (Foster et al. 2000; Wolff et al. 2000). A mean Fa value of 0.88 was reported by Ke et al. (2014). 
To describe the distribution, tissue: blood partition coefficients $(\mathrm{P})$ of methadone and EDDP were obtained by dividing tissue: plasma partition coefficients by the corresponding blood/plasma ratio (BPr) obtained from subjects on methadone maintenance treatment (Hsu et al. 2013), to correct for the differences in the distribution of the compounds in blood and plasma. The tissue: plasma partition coefficients of methadone and EDDP were calculated using prediction method 1 which applies the algorithms of Berezhkovskiy (2004) in the Simcyp Simulator V18 Release 1 (Certara, Sheffield, UK) requiring information on the fraction unbound in plasma $\left(\mathrm{f}_{\mathrm{u}, \mathrm{p}}\right)$, lipophilicity $(\log \mathrm{P})$ and acid-base properties $(\mathrm{pKa})$. The $\log \mathrm{P}$ and $\mathrm{pKa}$ value of methadone were obtained from literature (Gerber et al. 2001; Ke et al. 2014). The $\log P$ and pKa of EDDP were obtained from Marvinsketch (ChemAxon, Hungary). The $f_{u, p}$ of methadone was obtained from the in silico Simcyp prediction tool (Certara). The $f_{u, p}$ value of 0.3 for EDDP was obtained from the study of Moody et al. (2008). The $f_{u, p}$ of methadone and EDDP were also measured using pooled human plasma in the current study (see "RED assay" section). Since the influence of blood: tissue partition coefficients derived based on different $f_{u, p}$ values on the model output was negligible (data not shown), the blood: tissue partition coefficients calculated with the Simcyp-derived $f_{u, p}$ were used.

Liver was identified as the metabolizing organ in the PBK model since conversion of methadone was reported to primarily occur in the liver (Foster et al. 2004; Totah et al. 2008). Although Oda and Kharasch (2001) observed conversion of methadone in in vitro human intestinal microsomal incubations, the contribution of this intestinal metabolism to the elimination of methadone in vivo seems to be relatively small compared to the contribution of hepatic metabolism (Ke et al. 2014). Given that only minor methadone depletion was observed in the incubations with pooled intestinal microsomes (see "In vitro microsomal incubations" section), intestinal metabolism was not considered in the model. Conversion of EDDP to EMDP was not included in the model since no EMDP measured in the microsomal incubations with EDDP (see "In vitro microsomal incubations" section). The in vitro $V_{\max }$ obtained from human liver microsomal incubations were scaled to the in vivo situation taking the total liver microsomal protein yield of $32 \mathrm{mg}$ microsomal protein/ $\mathrm{g}$ liver into account (Barter et al. 2007).

After oral dosing, the urinary excretion of methadone and its metabolites accounts for up to $50 \%$ of the given dose (Ånggård et al. 1975; Lugo et al. 2005; Sullivan and Due 1973) with the ratio of unchanged methadone to EDDP ranging between 1/1 to 1/5 (Kharasch et al. 2004, 2009; Verebely et al. 1975). Therefore urinary excretion of methadone and EDDP was included in the PBK model. In addition, biliary excretion was included in the submodel of EDDP since 
the recovery of EDDP in faeces was reported to account for up to 39\% (Foster 2001). The renal clearance of methadone (RCLmet) was set at $1.45 \mathrm{l} / \mathrm{h}$ which was the average of the values reported in different in vivo studies (Boulton et al. 2001; Foster et al. 2000; Kharasch et al. 2009). The renal clearance (RCLeddp) and biliary excretion rate constant (kbile) of EDDP were obtained by the curve fitting option in Berkeley Madonna (version 8.3.18, UC Berkeley, CA, USA $)$ in which the steady-state blood maximum concentration $\left(\mathrm{C}_{\max }\right)$ of EDDP obtained with the PBK model was fitted to the steady-state blood $\mathrm{C}_{\max }$ of EDDP that was reported in subjects receiving methadone maintenance treatment with an oral dose of $57.5 \mathrm{mg} /$ day (De Vos et al. 1995). This resulted in the fitted rate constants for RCLeddp and Kbile of $19.99 \mathrm{l} / \mathrm{h}$ and $1.65 / \mathrm{h}$ (Table 1), respectively. Kinetic model calculations and curve fitting were performed with Berkeley Madonna, applying Rosenbrock's algorithms for solving stiff systems. Model equations were shown in supplementary materials 2 . Human physiological parameters used in the PBK model were obtained from Brown et al. (1997) (Table 1). Table 2 shows the physicochemical parameters of methadone and EDDP.

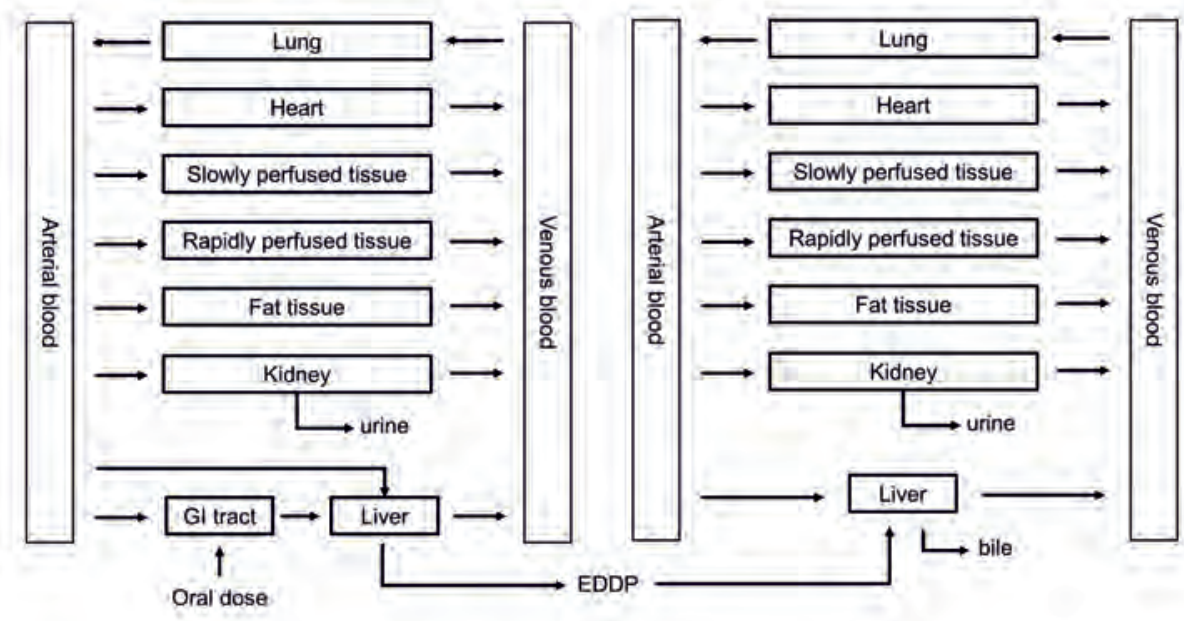

Fig. 3 Schematic diagram of the PBK model of methadone and EDDP 
Table 1 Physiological and biochemical parameters used in the PBK model for methadone and EDDP

\begin{tabular}{|c|c|c|c|}
\hline Parameters & Symbol & Value & References \\
\hline Body weight (kg) & BW & 70 & $\begin{array}{l}\text { Brown et al. } \\
\text { (1997) }\end{array}$ \\
\hline \multicolumn{3}{|l|}{ Tissue volume (\% body weight) } & \multirow{11}{*}{$\begin{array}{l}\text { Brown et al. } \\
\text { (1997) }\end{array}$} \\
\hline Liver & VLc & 0.0257 & \\
\hline Fat & $\mathrm{VFc}$ & 0.2142 & \\
\hline Lung & VLuc & 0.0076 & \\
\hline Arterial blood & VAc & 0.0198 & \\
\hline Venous blood & VVc & 0.0593 & \\
\hline Kidney & $\mathrm{VKc}$ & 0.004 & \\
\hline Heart & VHc & 0.0047 & \\
\hline Slowly perfused tissue & $\mathrm{VSc}$ & 0.5318 & \\
\hline Rapidly perfused tissue & VRc & 0.052 & \\
\hline Cardiac output (1/h) & Qc & 347.9 & \\
\hline \multicolumn{3}{|l|}{$\begin{array}{l}\text { Blood flow to tissue (\% cardiac } \\
\text { output) }\end{array}$} & \multirow[t]{7}{*}{$\begin{array}{l}\text { Brown et al. } \\
\text { (1997) }\end{array}$} \\
\hline Liver & QLc & 0.227 & \\
\hline Fat & $\mathrm{QFc}$ & 0.052 & \\
\hline Kidney & QKc & 0.175 & \\
\hline Heart & QHc & 0.04 & \\
\hline Slowly perfused tissue & QSc & 0.188 & \\
\hline Rapidly perfused tissue & QRc & 0.318 & \\
\hline \multirow{2}{*}{$\begin{array}{l}\text { Absorption rate constant of } \\
\text { methadone }(/ \mathrm{h})\end{array}$} & \multirow{2}{*}{ ka } & \multirow{2}{*}{0.59} & $\begin{array}{l}\text { Foster et al. } \\
(2000)\end{array}$ \\
\hline & & & $\begin{array}{l}\text { Wolff et al. } \\
(2000)\end{array}$ \\
\hline Fraction absorbed of methadone & $\mathrm{Fa}$ & 0.88 & Ke et al. (2014) \\
\hline Renal clearance of methadone $(1 / h)$ & RCLmet & 1.45 & $\begin{array}{l}\text { Boulton et al. } \\
(2001) \text {; Foster et } \\
\text { al. (2000); } \\
\text { Kharasch et al. } \\
(2009)\end{array}$ \\
\hline Renal clearance of EDDP (1/h) & RCLeddp & $19.99^{\mathrm{a}}$ & fitted values \\
\hline
\end{tabular}


Biliary excretion rate constant of $\operatorname{EDDP}(/ \mathrm{h})$ kbile $1.65^{\mathrm{a}}$ fitted values

${ }^{a}$ fitted value generated from EDDP data presented in the study of De Vos et al. (1995).

Table 2 Physicochemical parameters for methadone and EDDP

\begin{tabular}{llllllllllll}
\hline & & \multicolumn{8}{c}{ tissue: blood partition coefficients } \\
\cline { 5 - 11 } compound & LogP & pKa & BPr & liver & fat & $\begin{array}{l}\text { slowly } \\
\text { perfused } \\
\text { tissue }\end{array}$ & $\begin{array}{l}\text { rapidly } \\
\text { perfused } \\
\text { tissue }\end{array}$ & lung & kidney & heart \\
\hline methadone & $3.93^{\mathrm{a}}$ & $9.20^{\mathrm{b}}$ & $0.70^{\mathrm{c}}$ & 12.45 & 0.46 & 7.67 & 12.45 & 1.77 & 7.56 & 4.9 \\
EDDP & $4.63^{\mathrm{e}}$ & $9.64^{\mathrm{e}}$ & $0.87^{\mathrm{c}}$ & 11.51 & 0.18 & 7.06 & 11.51 & 1.56 & 6.95 & 4.48 \\
\hline
\end{tabular}

BPr, blood/plasma ratio. ${ }^{a}$ reported in Ke et al. (2014), ${ }^{\mathrm{b}}$ reported in Gerber et al. (2001), ${ }^{\mathrm{c}}$ reported in Hsu et al. (2013), ${ }^{d}$ obtained by dividing tissue: plasma partition coefficients by the corresponding BPr values, ${ }^{\mathrm{e}}$ obtained from Marvinsketch (ChemAxon)

\subsection{Evaluation of the PBK model}

To evaluate the performance of the PBK model developed, comparisons were made between predicted blood concentrations and area under the curve (AUC) values of methadone and in vivo blood concentrations and AUC values obtained in clinical studies with repeated daily oral administration at different doses of methadone. Given that the kinetics of methadone were reported based on plasma concentrations in clinical studies, the plasma concentration time curves were extracted from graphs presented in the respective clinical studies using GetData Graph Digitizer $2.26^{1}$ and further converted to blood concentration time curves by multiplying with the BPr value. For the evaluation of the PBK model, the model parameter body weight and the oral dose were chosen to match the values used in the clinical studies. The specifications of in vivo kinetic studies of methadone used to evaluate the PBK model are summarized in Table 4.

\subsection{Sensitivity analysis}

A local parameter sensitivity analysis was performed to identify influential parameters on the predicted $\mathrm{C}_{\max }$ in the heart venous blood during the steady state phase. The normalized sensitivity coefficient (SC) was calculated with the following equation (4):

\footnotetext{
${ }^{1}$ Available at: http://getdata-graph-digitizer.com [Accessed 20 ${ }^{\text {th }}$ November 2019]
} 


$$
\mathrm{SC}==\frac{\left(\mathrm{C}^{\prime}-\mathrm{C}\right)}{\left(\mathrm{P}^{\prime}-\mathrm{P}\right)} \times \frac{\mathrm{P}}{\mathrm{C}}
$$

where $\mathrm{C}$ is the initial value of the model output being the steady-state $\mathrm{C}_{\max }$ of the heart venous blood, $\mathrm{C}^{\prime}$ is the model output after a $1 \%$ increase in each model parameter value, $\mathrm{P}$ is the initial parameter value and $\mathrm{P}^{\prime}$ is the parameter value after a $1 \%$ increase. Parameters with an absolute SC $>0.1$ are considered to be influential on the model output (Chiu et al. 2007; Rietjens et al. 2011). The sensitivity analysis was carried out for a subject with a body weight of $70 \mathrm{~kg}$ (Brown et al. 1997) and for oral daily doses of 20 and $200 \mathrm{mg}$, representing respectively a clinically relevant dose level and a dose level associated with a high proportion of case reports of cardiotoxicity in subjects receiving methadone (Chou et al. 2014).

\subsection{Translation of in vitro concentration-response data to in vivo dose- response data using PBK modelling-based reverse dosimetry}

A change in the FPDc in the vitro field potential waveforms can be considered the surrogate endpoint for the QTc interval in the human ECG (Zwartsen et al. 2019). Based on this consideration, PBK modelling-based reverse dosimetry was applied to translate in vitro concentration-response data on FPDc obtained from the hiPSC-CM using the MEA to in vivo dose-response curves for QTc. To this purpose, the in vitro unbound concentrations of methadone tested in the hiPSC-CM MEA assay were set equal to the unbound steady-state $\mathrm{C}_{\max }$ of methadone in the heart venous blood by correcting the fraction unbound in plasma to a fraction unbound in blood using the BPr value in equation (5):

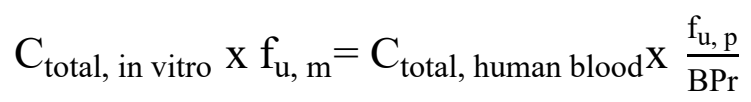

where $\mathrm{C}_{\text {total, in vitro }}$ and $\mathrm{f}_{\mathrm{u}, \mathrm{m}}$ are the in vitro methadone concentration and unbound fraction of methadone in the in vitro exposure medium, respectively. BPr is the blood to plasma ratio of methadone and $\mathrm{f}_{\mathrm{u}, \mathrm{p}}$ is the unbound fraction of methadone in human plasma. $\mathrm{C}_{\text {total, human blood }}$ values were extrapolated to in vivo oral doses by PBK-modeling based reverse dosimetry, using a bodyweight of $70 \mathrm{~kg}$ (Brown et al. 1997). The same procedure was performed for each of the in vitro concentrations tested in the MEA. Thus the entire in vitro concentration-response curve was translated to a predicted in vivo dose-response curve. 


\subsection{Evaluation of the PBK modelling-based reverse dosimetry approach}

To evaluate the performance of the PBK modelling-based reverse dosimetry approach, the predicted dose-response curves were compared to dose-response data for QTc prolongation obtained from published literature including single case reports, case series (Table S1), crosssectional, retrospective and prospective studies (Table S2). To better illustrate the dosedependent effect of methadone on QTc prolongation, individuals who have potential QTc prolonging risk factors including structural heart disease, electrolyte imbalance, hepatic impairment, concomitant use of medications that potentially prolong QTc or influence the metabolism of methadone (Stringer et al. 2009) were excluded from case reports and case series used for the evaluation. Similar criteria could not be applied to the cross-sectional, retrospective and prospective studies due to the absence of detailed individual information on these risk factors. Potential QTc prolonging risk factors and exclusion criteria for these studies were summarised in Table S2. To facilitate the comparison between in vitro and in vivo derived values, both the absolute FPDc values obtained from the in vitro cardiotoxicity assay and the in vivo methadone-induced QTc prolongation on ECG were expressed as relative percentages by dividing the post-treatment FPDc and QTc values by the respective baseline values. For the studies in which baseline QTc data were not reported, a population baseline QTc was assumed as described in the study of Florian et al. (2012) in which baseline QTc was set equal to baseline QTc values identified in Wedam et al. (2007), with an average value of $407 \mathrm{~ms}$ (411 ms for female; $405 \mathrm{~ms}$ for male).

\subsection{Benchmark Dose modelling}

BMD analysis of predicted in vivo dose-response curves was performed to derive a BMD that can be used as point of comparison to evaluate the predicted dose-response data against therapeutic methadone levels reported in the literature. The benchmark response (BMR) was defined as a $10 \%$ change compared to the control. For the QTc an effect of $10 \%$ change over the population baseline of $407 \mathrm{~ms}$, amounting to a QTc of $450 \mathrm{~ms}$ is frequently used as a threshold for abnormal QTc prolongation (Anchersen et al. 2009; Chou et al. 2014; ICH 2005b; Mujtaba et al. 2013; Treece et al. 2018). The BMD values resulting in a BMR of $10 \%$ with lower and upper $95 \%$ confidence limit were defined as $\mathrm{BMDL}_{10}$ and $\mathrm{BMDU}_{10}$. The European Food Safety Authority (EFSA) web-tool ${ }^{2}$ integrated with the R-package PROAST version

2 EFSA Statistical Models-BMD. [Online]. Available at: https://shiny-efsa.openanalytics.eu/app/bmd [Accessed 20 December , 2019] 
66.40 developed by the Dutch National Institute for Public Health and the Environment (RIVM) was used for BMD analysis. In short, the continuous data from the predicted in vivo doseresponse curves were fitted to a set of models including the Exponential, Hill, Inverse Exponential, and the Log-Normal Family models. According to the flow-chart described in the manual ${ }^{2}$ provided by EFSA, all fitted models excluding the FULL and NULL model were used for model averaging and a weighted average model was constructed to estimate model averaged confidence intervals using bootstrap sampling (Wheeler and Bailer 2007). Weighting was based on the model's Akaike's Information Criterion (AIC) values where models with lower AIC values get a larger weight. 200 bootstrap data sets were run to calculate the final BMD confidence intervals from model averaging.

In vitro concentration-response cardiotoxicity data were analysed using the same BMD approach to derive benchmark concentrations that induced a 20\% change in the FPDc over the control $\left(\mathrm{BMC}_{20}\right)$ for comparing the potency of methadone, EDDP and EMDP. The final $\mathrm{BMC}_{20}$ values were obtained by weighted averaging $\mathrm{BMC}_{20}$ values derived from all fitted models excluding the FULL and NULL model. For this analysis a BMR of $20 \%$ was chosen being the lowest BMR allowing reliable curve fitting.

\section{Results}

\subsection{In vitro cardiotoxicity in the hiPSC-CM MEA assay}

Fig. 4 shows the cardiotoxicity of methadone, EDDP and EMDP in hiPSC-CM as detected in the MEA. Methadone and its primary metabolite EDDP significantly prolonged the FPDc in a concentration-dependent manner with a $\mathrm{BMC}_{20}$ of $0.6 \mu \mathrm{M}$ and $2.3 \mu \mathrm{M}$, respectively. Of interest to note is that the secondary metabolite EMDP induced an opposite effect, shortening the FPDc in a concentration-dependent manner with the concentration shortening the FPDc by $20 \%$ amounting to $3.8 \mu \mathrm{M}$. Both methadone and EDDP induced arrhythmia-like waveforms from 3 $\mu \mathrm{M}$ onwards while cessation of beating was observed upon the treatment of the hiPSC-CM with methadone and EDDP at $30 \mu \mathrm{M}$. EMDP caused beating arrest in certain wells at $30 \mu \mathrm{M}$ without inducing arrhythmia-type waveforms within the test concentration range. The FPDc of hiPSC-CM treated with repeated application of $0.1 \%(\mathrm{v} / \mathrm{v})$ DMSO in the vehicle control well was not significantly affected (Fig. S3). 


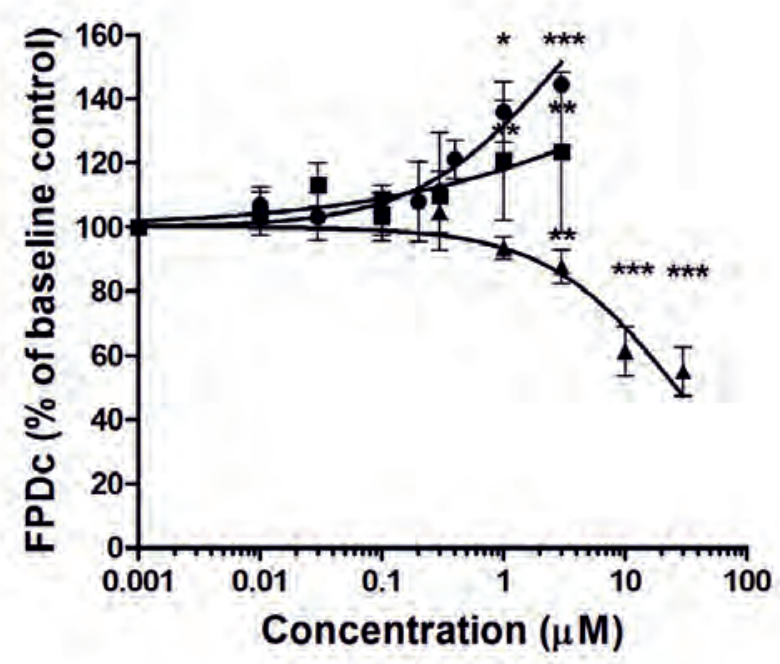

Fig. 4 Concentration-response curves for the effect of methadone (circles), EDDP (squares) and EMDP (triangles) on FPDc in hiPSC-CM detected by the MEA. The response of the baseline condition $(0.1 \%(\mathrm{v} / \mathrm{v}) \mathrm{DMSO})$ was set at $100 \%$. Data represent the mean of $4-9$ wells with in total 2638 electrodes. Each data point represents the mean \pm SD. Statistically significant changes in response compared to the solvent control are marked with ${ }^{*}$ with $p<0.05:{ }^{*}, p<0.01:{ }^{* *}$ and $p<0.001:{ }^{* * *}$

\subsection{In vitro microsomal incubations}

Fig. 5 shows the concentration-dependent formation rate of EDDP from methadone by human liver microsomes, which followed Michaelis-Menten kinetics. The apparent $\mathrm{V}_{\max }$ and $\mathrm{K}_{\mathrm{m}}$ values obtained from the data, and the catalytic efficiency $\left(\mathrm{V}_{\max } / \mathrm{K}_{\mathrm{m}}\right)$ are presented in Table 3 . No EMDP formation was measured in these incubations. In similar incubations using EDDP as the substrate, formation of EMDP was neither detectable. In incubations with intestinal microsomes applying the two highest methadone concentrations tested in liver microsomes $(1000$ and $1500 \mu \mathrm{M})$, formation of EDDP was less than $8 \%$ of the formation observed with liver microsomes at these concentrations. In addition, negligible formation of EDDP was observed in the incubation of $50 \mu \mathrm{M}$ methadone with increasing incubation time up to $120 \mathrm{~min}$ and protein concentrations up to $2 \mathrm{mg} / \mathrm{ml}$ human intestinal microsomal protein. Also in these incubations no EMDP formation was detected. This implied that conversion by intestinal microsomes was considered limited compared to conversion by human liver microsomes and therefore methadone conversion by intestinal tissue was not incorporated in the PBK model and hence no further kinetic constants were derived. 


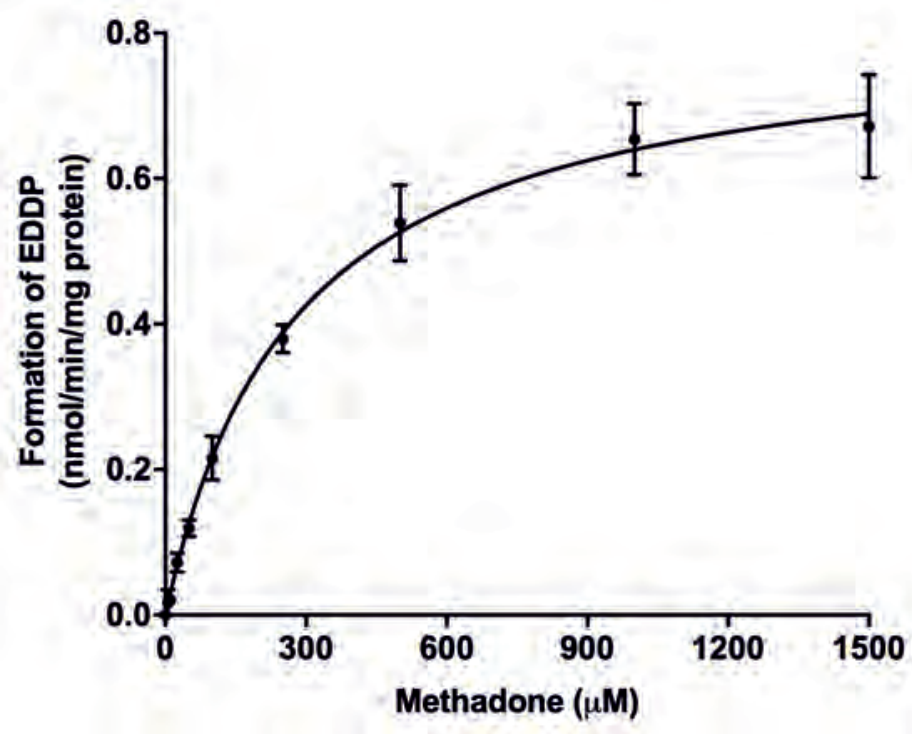

Fig. 5 Concentration-dependent formation of EDDP in incubations with human liver microsomes. Data represent the mean of 3 independent experiments. Each data point represents the mean \pm SD

Table 3 Kinetic constants for formation of EDDP from methadone obtained from in vitro incubations with human liver and intestinal microsomes

\begin{tabular}{lllllll}
\hline Organ & Substrate & Metabolite & $\begin{array}{l}\mathrm{V}_{\max } \pm \\
(\mathrm{nmol} / \mathrm{min} / \mathrm{mg} \\
\text { microsomal protein })\end{array}$ & $\begin{array}{l}\mathrm{K}_{\mathrm{m}} \pm \mathrm{SD} \\
(\mu \mathrm{M})\end{array}$ & $\begin{array}{l}\text { Catalytic efficiency a } \\
(\mu 1 / \mathrm{min} / \mathrm{mg} \text { microsomal } \\
\text { protein })\end{array}$ \\
\hline \multirow{2}{*}{ liver } & methadone & EDDP & $0.82 \pm 0.026$ & 26.78 & 2.97 \\
& & EMDP & n.d. & n.d. & - \\
& EDDP & EMDP & n.d. & n.d. & - \\
\hline intestine & methadone & EDDP & $0.058,0.057^{\text {b }}$ & n.d. & - \\
& & EMDP & n.d. & n.d. & - \\
\hline
\end{tabular}

n.d., not determined, since EDDP and EMDP were unable to be quantified (see text for details); -, unable to calculate. ${ }^{a} \mathrm{~V}_{\max } / \mathrm{K}_{\mathrm{m}} * 1000,{ }^{\mathrm{b}}$ formation rate at $1000 \mu \mathrm{M}$ and $1500 \mu \mathrm{M}$

\subsection{Unbound fraction for methadone in in vitro hiPSC-CM MEA medium and in human plasma}

Due to the use of serum free medium in the hiPSC-CM MEA assay, the unbound fraction of methadone in the in vitro medium was relatively high, amounting to $0.79 \pm 0.041$ compared to the unbound fraction in pooled human plasma determined to be $0.055 \pm 0.011$. The unbound 
fraction of EDDP in the in vitro medium was $0.90 \pm 0.072$ and was $0.30 \pm 0.015$ in pooled human plasma.

Considering the large inter-individual variation in plasma protein binding for methadone observed in in vivo studies (Eap et al. 1990; Olsen 1973; Romach et al. 1981; Wilkins et al. 1997), also two extreme $f_{u, p}$ values $(0.034$ and 0.22$)$ obtained from the literature together with the Simcyp-derived and RED-derived $f_{u, p}$ values ( 0.15 and 0.055 , respectively) were used to translate in vitro effect-concentrations to the total blood concentration as presented in equation 5, which were subsequently subject to PBK modelling-based reverse dosimetry.

\subsection{PBK model development and evaluation}

To evaluate the performance of the human PBK model, the predicted methadone blood kinetics were compared to in vivo human data obtained from the literature. The specifications of in vivo studies on the subjects receiving methadone maintenance treatment that are used for the PBK model evaluation are summarized in Table 4. As illustrated in Fig. 6, the developed PBK model accurately predicts the change of methadone blood concentrations during the last 24 hours upon repeated oral methadone exposure as described in the study of Foster et al. (2000) and Liu et al. (2007). Table 4 further shows the detailed comparison between the model prediction and the in vivo kinetic data using steady-state blood $\mathrm{C}_{\max }$ and AUC values on the last day of exposure as model outcomes. For methadone the predicted kinetic values are in accordance with reported values expressing a 0.78 - to 1.35 -fold difference in $\mathrm{C}_{\max }$ values and 0.76- to 0.97 fold difference in AUC values (Table 4).

Table 4 Summary of in vivo kinetic studies and evaluation of the PBK model predictions for methadone steady-state blood $\mathrm{C}_{\max }$ and $\mathrm{AUC}$ values based on the data derived from in vivo kinetic studies

\begin{tabular}{|c|c|c|c|c|c|c|c|c|}
\hline $\begin{array}{l}\text { Mean } \\
\text { body } \\
\text { weight } \\
(\mathrm{kg})\end{array}$ & $\begin{array}{l}\text { Mean } \\
\text { methadone } \\
\text { dose }^{\text {a }} \\
(\mathrm{mg} / \text { day) }\end{array}$ & $\begin{array}{l}\text { In vivo } \\
\mathrm{C}_{\max } \\
(\mathrm{ng} / \mathrm{ml})^{\mathrm{b}}\end{array}$ & $\begin{array}{l}\text { In vivo } \\
\text { AUC } \\
(\mathrm{ng} \cdot \mathrm{h} / \mathrm{ml})^{\mathrm{b}}\end{array}$ & $\begin{array}{l}\text { Predicted } \\
\mathrm{C}_{\max } \\
(\mathrm{ng} / \mathrm{ml})\end{array}$ & $\begin{array}{l}\text { Predicted } \\
\text { AUC } \\
(\mathrm{ng} \cdot \mathrm{h} / \mathrm{ml})\end{array}$ & $\begin{array}{l}\text { Ratio } \\
\text { predicted } \\
\mathrm{C}_{\max } / \text { in } \\
\text { vivo } \mathrm{C}_{\max }\end{array}$ & $\begin{array}{l}\text { Ratio } \\
\text { predicted } \\
\text { AUC/in } \\
\text { vivo AUC }\end{array}$ & Reference \\
\hline 74 & 70 & 346.2 & 5097 & 320.5 & 4967 & 0.93 & 0.97 & $\begin{array}{l}\text { Foster et } \\
\text { al. }(2000) \\
\text { c }\end{array}$ \\
\hline 90 & 100 & 453.6 & 7889 & 385.2 & 5969 & 0.85 & 0.76 & $\begin{array}{l}\text { Liu et al. } \\
(2007)^{c}\end{array}$ \\
\hline $70^{d}$ & 61 & 216.0 & n.r. & 293.1 & 4542 & 1.35 & - & $\begin{array}{l}\text { Diong et } \\
\text { al. (2014) }\end{array}$ \\
\hline
\end{tabular}




$\begin{array}{lllllllll}64.7 & 57.5 & 383.6 & 5978 & 296.4 & 4591 & 0.78 & 0.77 & \begin{array}{l}\text { De Vos et } \\ \text { al. (1995) }\end{array}\end{array}$

n.r., not reported; -, unable to calculate; ${ }^{\mathrm{a}}$ free base form of methadone, ${ }^{\mathrm{b}}$ blood data were obtained by multiplying reported plasma data by the BPr value, ${ }^{c}$ in vivo $\mathrm{C}_{\max }$ and AUC is the sum of data of enantiomers, ${ }^{\mathrm{d}}$ the body weight of subjects was set equal to the value used in the PBK model since body weight of study subjects was not reported
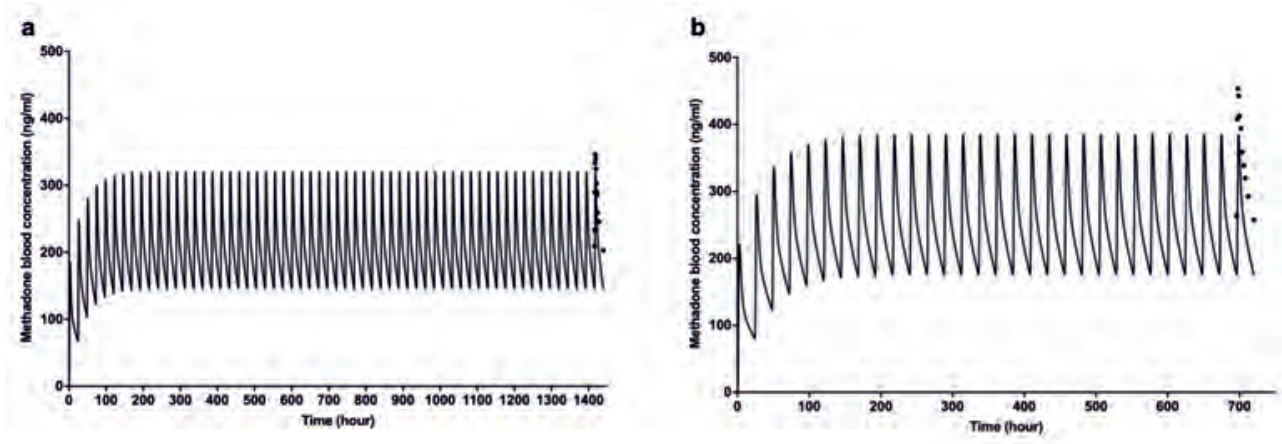

Fig. 6 Blood concentration-time curves of methadone in human predicted with the PBK model (lines) and published in vivo data (dots) after a repeated oral dose of $70 \mathrm{mg} / \mathrm{day}$ for 60 days (a) (Foster et al. 2000) and $100 \mathrm{mg} / \mathrm{day}$ for 30 days (b) (Liu et al. 2007)

\subsection{Sensitivity analysis}

Fig. 7 shows the most influential model parameters for the prediction of steady-state $C_{\max }$ in the heart venous blood upon exposure to oral repeated methadone doses of 20 and $200 \mathrm{mg}$. The results indicate that the normalized sensitivity coefficients of all PBK model parameters were not dose-dependent until at least $200 \mathrm{mg}$ /day and that the predicted steady-state $\mathrm{C}_{\max }$ in the heart venous blood is most sensitive to the oral fraction absorbed and the body weight with normalized SC values above 0.8 . The parameters related to liver metabolism (volume of liver, liver microsomal protein yield, unscaled maximum rate of methadone metabolism) also substantially influence the model outcome with normalized SC values of 0.6. The absorption rate constant and the partition coefficient rapidly perfused tissue to blood of methadone are less influential with normalized $\mathrm{SC}$ value of 0.25 and 0.1 , respectively. 


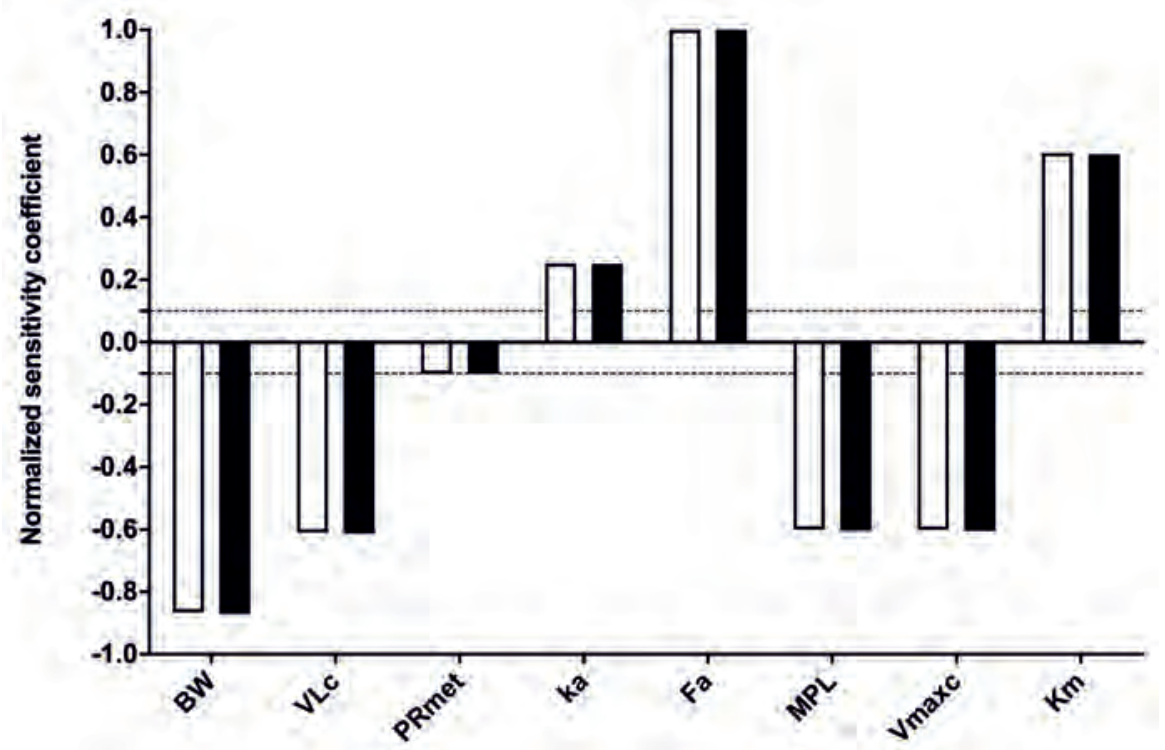

Fig. 7 Normalized SCs of PBK model parameters for the prediction of steady-state $C_{\max }$ of methadone in the heart venous blood upon oral repeated doses of $20 \mathrm{mg} / \mathrm{day}$ (white bars) and 200 mg/day (black bars). Model parameters with normalized SC with an absolute value higher than 0.1 (dotted lines) are shown. BW, body weight; VLc, fraction of liver; PRmet, partition coefficient rapidly perfused tissue: blood of methadone; ka, absorption rate constant; Fa, oral fraction absorbed; MPL, liver microsomal protein yield; Vmaxc, unscaled maximum rate of methadone metabolism in liver; Km, Michaelis-Menten constant for methadone metabolism in liver

\subsection{Translation of in vitro concentration-response data into in vivo dose- response data using PBK-modelling based reverse dosimetry}

Although EDDP induced concentration-dependent prolongation of FPDc in the in vitro assay, the free blood $\mathrm{C}_{\max }$ of EDDP, after an oral dose of $57.5 \mathrm{mg} /$ day, was estimated to be $0.05 \mu \mathrm{M}$ based on EDDP data reported in De Vos et al. (1995). By using the current PBK model, the free blood $\mathrm{C}_{\max }$ of EDDP was predicted to be $0.17 \mu \mathrm{M}$ at a relatively high dose level of methadone of $200 \mathrm{mg} /$ day. Both the reported and predicted free blood $\mathrm{C}_{\max }$ of EDDP are substantially lower than unbound concentrations causing cardiotoxicity in the hiPSC-CM MEA assay (unbound $\mathrm{BMC}_{20}=2.07 \mu \mathrm{M}$ ) (Fig. 4). To reach the unbound $\mathrm{BMC}_{20}$ value of $2.07 \mu \mathrm{M}$, a methadone dose level of $2600 \mathrm{mg}$ /day was estimated to be required, which is 22-fold higher than the highest clinical relevant dose of $120 \mathrm{mg} /$ day (Chou et al. 2014). Therefore, the cardiotoxicity of EDDP was not considered to play a role in methadone induced cardiotoxicity and thus also not considered for the reverse dosimetry. 
Upon correction for protein binding performed using the values for $f_{u, m}$ and $f_{u, p}$ described above, the in vitro concentration-response curve of methadone obtained in the hiPSC-CM as detected by the MEA was translated to in vivo dose-response curves for human cardiotoxicity using the developed PBK model. As mentioned in the "unbound fraction for methadone" section, $f_{u . m}$ of 0.79 was used to correct for protein binding of methadone in the in vitro medium while for the in vivo situation four different $f_{u, p}$ values were used including the experimental $f_{u, p}$ value obtained from pooled human plasma, an in-silico derived $f_{u, p}$ value and two extreme $f_{u, p}$ values obtained from the literature (Eap et al. 1990; Foster et al. 2000; Moody et al. 2008; Olsen 1973; Romach et al. 1981; Wilkins et al. 1997). This resulted in 4 predicted in vivo doseresponse curves for methadone induced cardiotoxicity, one for each of the $f_{u, p}$ values (Fig. 8). These predicted dose-response curves were subsequently compared to available in vivo human data.

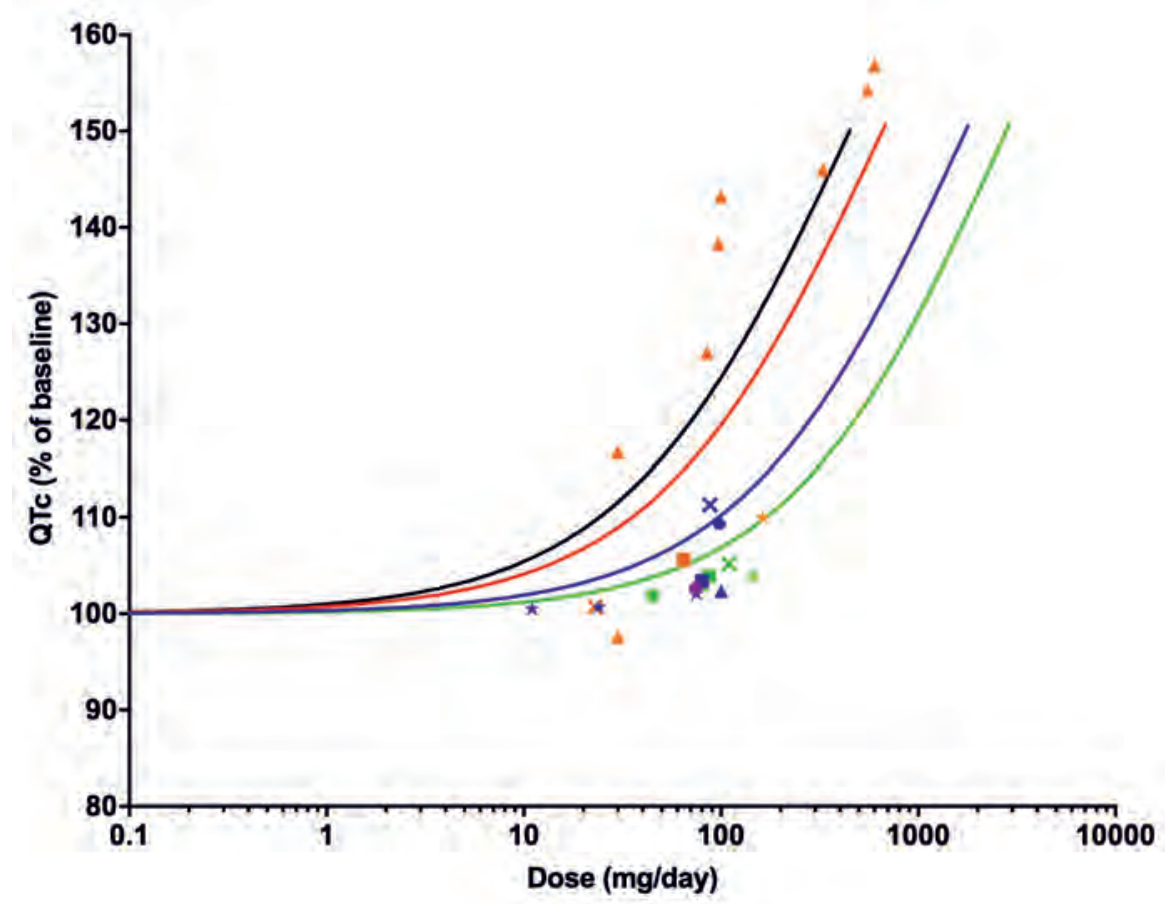

Fig. 8 Predicted dose-response curves for cardiotoxicity of methadone obtained by using PBK modelling-based reverse dosimetry compared to in vivo dose-response data derived from literature. The curves represent the prediction based on a $\mathrm{f}_{\mathrm{u}, \mathrm{p}}$ of 0.22 (black line), 0.15 (red line), 0.055 (blue line) and 0.034 (green line). Symbols represent the data obtained from case reports, case series of individuals (orange triangles) (Esses et al. 2008; Fredheim et al. 2006; Krantz et al. 2002) and other studies as follows: Bart et al. (2017) (purple circle); Carlquist et al. (2015) (orange square); Chang et 
al. (2012) (green circle); Chowdhury et al. (2015) (dark blue cross); Cruciani et al. (2005) (green cross); Eap et al. (2007) (green star); Ehret et al. (2006) (dark blue triangle); Fareed et al. (2013) (dark blue circle); Heesch et al. (2015) (dark blue star); Krantz et al. (2005) (orange circles); Maremmani et al. (2005) (green square); Martell et al. (2005) (green triangle); Peles et al. (2007) (orange star); Reddy et al. (2010) (orange circles); Roy et al. (2012) (dark blue square). The in vivo data are summarized in Table S1 and S2

\subsection{Evaluation of the PBK modelling-based reverse dosimetry approach and BMD analysis of predicted dose-response data}

To evaluate the performance of the PBK modelling-based reverse dosimetry approach, the dose-response data for QTc prolongation obtained from case reports, case series, crosssectional, retrospective and prospective studies were compared with the predicted doseresponse curves for QTc prolongation taking different $f_{u, p}$ values into account. This comparison, presented in Fig. 8, reveals that the predicted in vivo dose-response curves for QTc prolongation were comparable with reported in vivo data. The prediction of QTc prolongation with the $f_{u, p}$ value of 0.15 obtained from Simcyp is best in line with the majority of reported QTc prolongation data of individual cases. The QTc prolongation data reported in population studies, however, were more close to the predicted dose-response curve with the $f_{u, p}$ value of 0.055 obtained from the RED assay.

To further evaluate the model predictions a BMD analysis was performed. $\mathrm{BMDL}_{10}$ values were derived and used as points of comparison. Fig. 9 presents the $\mathrm{BMDL}_{10}$ derived from the dose-response curves presented in Fig. 8, predicted with the different $f_{u, p}$ values while also presenting therapeutic dose levels of methadone. The comparison presented in Fig. 9 reveals that the predicted $\mathrm{BMDL}_{10}$ values overlap with the therapeutic methadone dose levels. The predicted $B M D L_{10}$ values for methadone induced cardiotoxicity based on high $\mathrm{f}_{\mathrm{u}, \mathrm{p}}$ values of 0.22 and 0.15 are 1.7- and 2.4-fold higher respectively, than the recommended initial dose for opioid-native patients (10 mg/day), and the predicted $B_{M D L} 10$ values based on low $f_{u, p}$ values of 0.055 and 0.034 are 2.2- and 3.6-fold higher respectively than the recommended initial dose for opioid users (30 mg/day) (Chou et al. 2014; BCCSU 2017). This indicating that these therapeutic dose levels are below the dose levels predicted to result in $10 \%$ change, an effect size that can be used as a threshold to evaluate abnormal QTc prolongation (Anchersen et al. 2009; Chou et al. 2014; ICH 2005b; Mujtaba et al. 2013; Treece et al. 2018). The maintenance dose of 60-120 mg methadone/day (Chou et al. 2014; BCCSU 2017) is however 0.6 to 7.2-fold 
higher than the predicted $\mathrm{BMDL}_{10}$ values in all scenarios, pointing at a potential cardiotoxic effect in especially individuals with relatively lower plasma protein binding (higher $f_{u, p}$ ). Detailed information on the BMD analysis can be found in the supplementary materials 1 Table S3-S7 and the BMD values are summarized in Table S7.

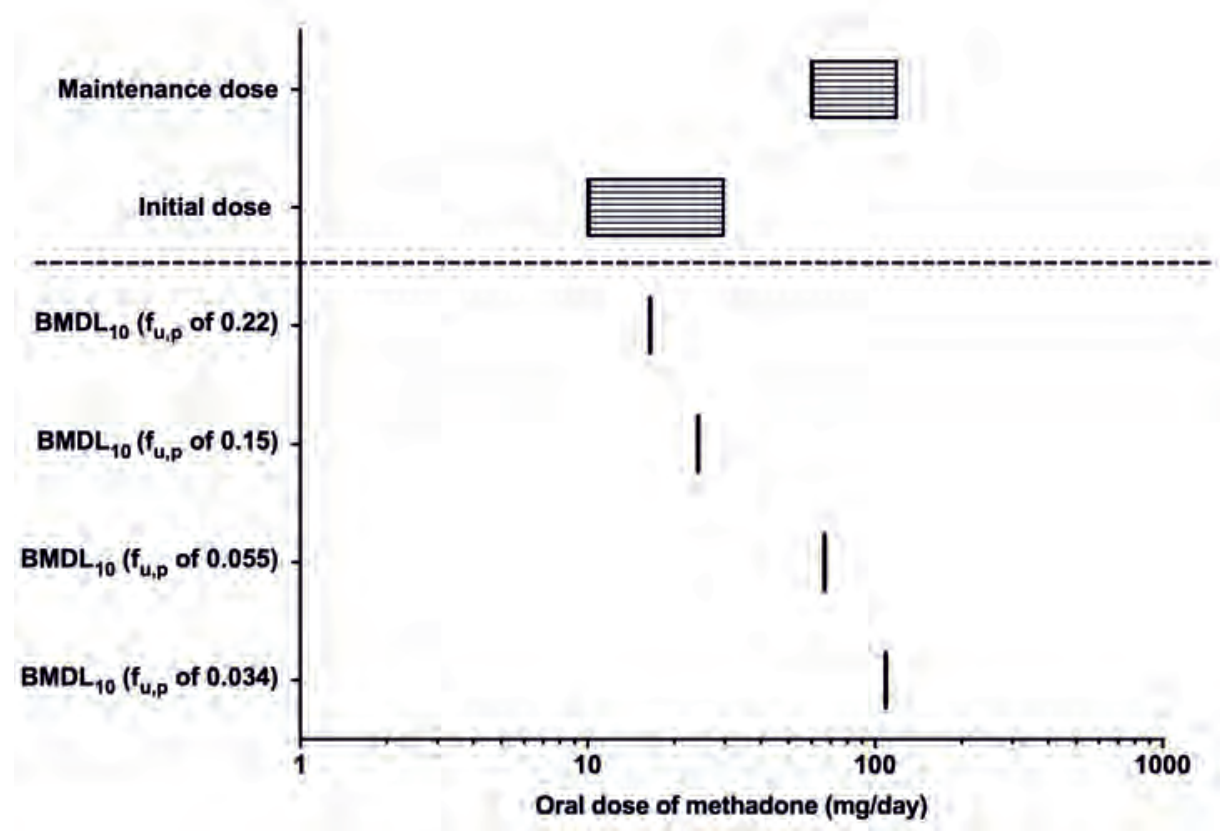

Fig. 9 Comparison of BMDL values derived from the predicted dose-response curves for human cardiotoxicity of methadone presented in Fig. 8 (lines) and therapeutic dose levels reported in the literature (boxes filled with horizontal lines)

\section{Discussion}

The aim of the present study was to investigate whether human in vivo cardiotoxicity could be predicted by a novel testing strategy combining the in vitro toxicity assay with hiPSC-CM in a MEA and PBK modelling-based reverse dosimetry. Methadone was used as the model compound given that for this drug both kinetic and clinical human data for evaluation of predictions made were available.

The in vitro electrophysical cardiotoxicity was detected using hiPSC-CM combined with the MEA technology, which can capture the overall effects on multiple ion channels on the extracellular field potential. The change in FPDc in the in vitro obtained field potential waveforms, can be considered a surrogate endpoint for the QTc interval in the human ECG (Zwartsen et al. 2019), the parameter known to be indicative for methadone induced 
cardiotoxicity (Mujtaba et al. 2013). The results show that methadone induced a concentrationdependent prolongation of FPDc which is in line with the study of Kuryshev et al. (2010) reporting that methadone prolonged the action potential duration using patch clamp recordings in human cardiomyocytes. Studies using mammalian cells transfected with cardiac ion channels revealed that the prolonged effect on FPDc can be ascribed to the inhibition of the hERG and sodium channels (Eap et al. 2007; Kuryshev et al. 2010). The major methadone metabolite EDDP appeared to also prolong the FPDc albeit with lower potency than methadone. This lower potency of EDDP is in line with the fact that EDDP was reported to be a weaker hERG channel blocker compared to methadone (Eap et al. 2007; Katchman et al. 2002) while effects on other ion channels such as sodium channel may contribute to the observed EDDPinduced FPDc prolongation effect (Mishra et al. 2014). Neither FPDc prolongation nor arrhythmia-type waveforms were observed upon exposure of the hiPSC-CM to EMDP which is in accordance with the previous study reporting EMDP to not inhibit hERG channels (Eap et al. 2007). Given that the in vivo total plasma concentration of EMDP has been reported to be less than $0.04 \mu \mathrm{M}$ after clinical relevant dosing (Alburges et al. 1996), it can be concluded that the in vitro effects of EMDP in the hiPSC-CM MEA assay, with an in vitro $\mathrm{BMC}_{20}$ for decreasing FPDc of $3.8 \mu \mathrm{M}$, would not be relevant in in vivo. Thus, the methadone-induced prolongation of the FPDs is unlikely to be counteracted by EMDP and the cardiotoxicity of EMDP was not further taken into account.

The evaluation of the developed PBK model against literature data available on steady-state blood $\mathrm{C}_{\max }$ and AUC values of methadone (De Vos et al. 1995; Diong et al. 2014; Foster et al. 2000; Liu et al. 2007), indicated that the model was able to adequately predict the kinetics of methadone with differences being less than 2-fold, which is generally accepted as an adequate predictive performance (Badhan et al. 2019; WHO 2010).

It is generally assumed that the unbound concentration is responsible for the clinical response of a drug (Smith et al. 2010). Given that methadone is a lipophilic drug with basic properties, the extent of protein binding may play an important role in determining the free concentration and influence the therapeutic or toxic effects of methadone. Given that in vivo experimental data report variation in the $f_{u, p}$ values of methadone, the PBK modelling-based reverse dosimetry was performed taking into account different values for $f_{u, p}$. It is reported that the fraction of unbound methadone is significantly correlated to the plasma concentration of alpha1-acid glycoprotein (AAG) (Abramson 1982; Yang et al. 2006), which is known to be influenced by physiological and pathologic conditions of the subject (Eap et al. 1990). The 1.5- 
up to 6.5-fold difference in the four $\mathrm{f}_{\mathrm{u}, \mathrm{p}}$ values used for the PBK modelling-based reverse dosimetry are in line with the 2 to 20 -fold variation of the AAG concentration among individuals (Taguchi et al. 2013).

The predicted dose-response curves obtained from the PBK modelling-based reverse dosimetry, using the respective $f_{u, p}$ values were in line with in vivo data available from case reports, case series, cross-sectional, retrospective and prospective studies on methadone induced effects on in vivo QTc prolongation available in the literature (Fig. 8). This further validates the developed PBK model and provides support for the novel in vitro-in silico testing strategy for prediction of cardiotoxicity in human.

It is of interested to note that the predictions with high $f_{u, p}$ values $(0.22$ and 0.15$)$ are more in line with data obtained from individual case series while the data obtained with lower $f_{u, p}$ values $(0.055$ and 0.034$)$ especially match the data from population studies. The reasons underlying this observation remain to be elucidated but may be related to the fact that the concentration of AAG increases under the conditions of heroin addiction (Garrido et al. 2000), HIV infection (Barrail-Tran et al. 2010), and cancer (Huang and Ung 2013). Given that the subjects in the epidemiological studies were associated with those physiological and pathologic conditions, smaller $f_{u, p}$ values would be expected while the individual cases series were selected using criteria that specifically exclude these potential factors that interfere with the concentration of AAG.

To further evaluate the in vitro-in silico predictions for human cardiotoxicity of methadone, $\mathrm{BMDL}_{10}$ values derived from predicted dose-response curves were compared to therapeutic doses. The $\mathrm{BMDL}_{10}$ values appeared to overlap with the therapeutic dose levels. Given the fact that a $\mathrm{BMDL}_{10}$ value is generally considered a dose level that is comparable to a no observed adverse effect level (EFSA 2017), and 10\% effect is an effect size used as a threshold to evaluate abnormal QTc prolongation (Anchersen et al. 2009; Chou et al. 2014; ICH 2005b; Mujtaba et al. 2013; Treece et al. 2018), doses lower than the predicted BMDL 10 values would be expected to be without an effect on QTc prolongation, which is in line with the observation that the predicted BMDL 10 values based on high $f_{u, p}$ values and low $f_{u, p}$ values are 2- to 3-fold higher than the recommended initial dose for opioid-native patients $(10 \mathrm{mg} / \mathrm{day})$ and opioid users (30 mg/day), respectively (Chou et al. 2014; BCCSU 2017). The fact that the BMDL 10 values obtained with the relatively higher $f_{u, p}$ values are 2.5 to 7 -fold lower than the maintenance dose (60 mg/day), may explain the QTc prolongation observed in some methadone maintenance treatment patients given these therapeutic maintenance dose levels. 
This confirms the need for particular cautions (intensive ECG monitoring and determining arrhythmia risk factor) for patients receiving high doses of methadone (>100 mg) (Florian et al. 2012; Mujtaba et al. 2013; Treece et al. 2018). Krantz et al. (2002) reported that methadone induced TdP in patients, without the presence of other risk factors, prescribed an average dose of $400 \mathrm{mg} / \mathrm{day}$, which is consistent with our predictions given the fact that this dose is even 4fold higher than the $\mathrm{BMDL}_{10}$ value $(109 \mathrm{mg} /$ day) derived from the predictions based on the lowest $f_{u, p}$ values. The results of our study indicate that especially subjects with lower levels of plasma protein binding (higher $\mathrm{f}_{\mathrm{u}, \mathrm{p}}$ ) of methadone may be a group at extra risk.

The results of the present study indicate that $f_{u, p}$ may be a key parameter causing interindividual differences in the cardiotoxicity of methadone. The exact magnitude of the effect of changes in protein binding on toxicity, however, is not always straightforward since this is an interplay between the available fraction at the site of action, metabolism and excretion and may i.e. require detailed information on the fate of a compound within cells/the human body which is often not available. Moreover, the variability in other factors that influence the concentration in the heart venous blood may also cause variation in cardiotoxic effects in individuals. Based on the sensitivity analysis, the $\mathrm{C}_{\max }$ in heart venous blood is also influenced by metabolism-related parameters. A major enzyme involved in the metabolism of methadone to EDDP is CYP2B6, a cytochrome P450 that shows large interindividual variability due to genetic polymorphism (Kharasch 2017). It would be of interest to integrate also this variability in the PBK model-based reverse dosimetry approach and predict its influence on the in vivo effects of methadone. This is a topic beyond the aim of the present study, that is currently under investigation. In addition, given that methadone is the racemic mixture of R-and S-methadone and the latter enantiomer is mainly responsible for the cardiotoxic effects (Ansermot et al. 2010; Eap et al. 2007; Lin et al. 2009), it would also be of interest to predict methadone induced cardiotoxicity distinguishing between the R- and S-enantiomers.

In the present study we demonstrated the integration of the hiPSC-CM MEA data and PBK modelling-based reverse dosimetry to assess the in vivo cardiotoxicity of methadone in human. This in vitro-in silico approach enabled the translation of the in vitro concentration-response data on cardiotoxicity to predicted in vivo dose-response data for methadone-induced QTc prolongation in human. Comparison of model predictions to in vivo data revealed that the novel testing strategy provided adequate predictions for both in vivo kinetics and cardiotoxicity of methadone, also pinpointing to an important role for binding to plasma proteins in determining potential interindividual differences in sensitivity towards the cardiotoxic effects of methadone. 
The present study provides a proof-of-principle of using PBK modelling-based reverse dosimetry for QIVIVE to predict cardiotoxicity in human, providing a novel testing strategy for cardiac safety.

\section{Acknowledgements}

The authors acknowledge Tessa de Korte (Ncardia, Leiden, The Netherlands) for helpful discussions on the in vitro cardiotoxicity model and Marije Wagenaar (Van Hall Larenstein University of Applied Sciences, Leeuwarden, The Netherlands) for preparing the incubation protocol and work on the Berkeley Mad onna model code at an initial phase of the project.

This work was funded by a Grant from the China Scholarship Council (No. 201607720029 to MIAOYING SHI).

\section{Conflicts of interest:}

All authors declare that they have no conflict of interest.

\section{Supplementary data}

Supplementary data to this article can be found online at:

https://doi.org/10.1007/s00204-020-02766-7 


\section{References}

Abdullah R, Alhusainy W, Woutersen J, Rietjens IMCM, Punt A (2016) Predicting points of departure for risk assessment based on in vitro cytotoxicity data and physiologically based kinetic (PBK) modeling: the case of kidney toxicity induced by aristolochic acid I Food and chemical toxicology 92:104-116

Abramson FP (1982) Methadone plasma protein binding: Alterations in cancer and displacement from $\alpha 1$-acid glycoprotein Clinical Pharmacology \& Therapeutics 32:652-658

Alburges ME, Huang W, Foltz RL, Moody DE (1996) Determination of methadone and its N-demethylation metabolites in biological specimens by GC-PICI-MS Journal of analytical toxicology 20:362-368

Alinejad S, Kazemi T, Zamani N, Hoffman RS, Mehrpour O (2015) A systematic review of the cardiotoxicity of methadone EXCLI journal 14:577

Anchersen K, Clausen T, Gossop M, Hansteen V, Waal H (2009) Prevalence and clinical relevance of corrected QT interval prolongation during methadone and buprenorphine treatment: a mortality assessment study Addiction 104:993-999

Ando $\mathrm{H}$ et al. (2017) A new paradigm for drug-induced torsadogenic risk assessment using human iPS cell-derived cardiomyocytes Journal of pharmacological and toxicological methods 84:111-127

Ånggård E, Gunne L-M, Holmstrand J, McMahon RE, Sandberg C-G, Sullivan HR (1975) Disposition of methadone in methadone maintenance Clinical Pharmacology \& Therapeutics 17:258-266

Ansermot $\mathrm{N}$ et al. (2010). Substitution of (R, S)-methadone by (R)-methadone: impact on QTc interval. Archives of internal medicine, 170(6), 529-536.

Asakura K et al. (2015) Improvement of acquisition and analysis methods in multi-electrode array experiments with iPS cell-derived cardiomyocytes Journal of pharmacological and toxicological methods 75:17-26

Badhan RK, Gittins R, Al Zabit D (2019) The optimization of methadone dosing whilst treating with rifampicin: A pharmacokinetic modeling study Drug and alcohol dependence 200:168-180

Barrail-Tran A et al. (2010) Influence of alpha-1 glycoprotein acid concentrations and variants on atazanavir pharmacokinetics in HIV-infected patients included in the ANRS 107 trial Antimicrobial agents and chemotherapy 54:614-619

Bart G, Wyman Z, Wang Q, Hodges JS, Karim R, Bart BA (2017) Methadone and the QTc interval: paucity of clinically significant factors in a retrospective cohort Journal of addiction medicine 11:489

Barter ZE et al. (2007) Scaling factors for the extrapolation of in vivo metabolic drug clearance from in vitro data: reaching a consensus on values of human micro-somal protein and hepatocellularity per gram of liver Current drug metabolism 8:33-45

Bell SM et al. (2018) In vitro to in vivo extrapolation for high throughput prioritization and decision making Toxicology In Vitro 47:213-227

Berezhkovskiy LM (2004) Determination of volume of distribution at steady state with complete consideration of the kinetics of protein and tissue binding in linear pharmacokinetics Journal of pharmaceutical sciences 93:364-374

Bernauer U, Oberemm A, Madle S, Gundert-Remy U (2005) The use of in vitro data in risk assessment Basic \& clinical pharmacology \& toxicology 96:176-181

Blaauboer BJ (2010) Biokinetic modeling and in vitro-in vivo extrapolations Journal of Toxicology and Environmental Health, Part B 13:242-252 
Boulton DW, Arnaud P, DeVane CL (2001) Pharmacokinetics and pharmacodynamics of methadone enantiomers after a single oral dose of racemate Clinical Pharmacology \& Therapeutics 70:48-57

British Columbia Centre on Substance Use (BCCSU) (2017) A Guideline for the Clinical Management of Opioid Use Disorder. https://www.bccsu.ca/wp-content/uploads/2017/06/BC-OUD-Guidelines_June2017.pdf. Accessed 20 November 2019

Brown RP, Delp MD, Lindstedt SL, Rhomberg LR, Beliles RP (1997) Physiological parameter values for physiologically based pharmacokinetic models Toxicology and industrial health 13:407-484

Carlquist JF et al. (2015) A possible mechanistic link between the CYP2C19 genotype, the methadone metabolite ethylidene-1, 5-dimethyl-3, 3-diphenylpyrrolidene (EDDP), and methadone-induced corrected QT interval prolongation in a pilot study Molecular diagnosis \& therapy 19:131-138

Chang KC et al. (2012) Gender-specific differences in susceptibility to low-dose methadone-associated QTc prolongation in patients with heroin dependence Journal of cardiovascular electrophysiology 23:527-533

Chiu WA et al. (2007) Evaluation of physiologically based pharmacokinetic models for use in risk assessment Journal of Applied Toxicology: An International Journal 27:218-237

Chou R et al. (2014) Methadone safety: a clinical practice guideline from the American Pain Society and College on Problems of Drug Dependence, in collaboration with the Heart Rhythm Society The Journal of Pain $15: 321-337$

Chowdhury M, Wong J, Cheng A, Khilkin M, Palma E (2015) Methadone Therapy in Underserved Urban Community: QT c Prolongation and Life-Threatening Ventricular Arrhythmias Cardiovascular therapeutics 33:127-133

Clements M, Millar V, Williams AS, Kalinka S (2015) Bridging functional and structural cardiotoxicity assays using human embryonic stem cell-derived cardiomyocytes for a more comprehensive risk assessment Toxicological Sciences 148:241-260

Cruciani RA et al. (2005) Measurement of QTc in patients receiving chronic methadone therapy Journal of pain and symptom management 29:385-391

De Vos J, Ufkes J, van Wilgenburg H, Geerlings P, van den Brink W (1995) Pharmacokinetics of methadone and its primary metabolite in 20 opiate addicts European journal of clinical pharmacology 48:361-366

Diong SH et al. (2014) Quantitation of methadone and metabolite in patients under maintenance treatment Journal of analytical toxicology 38:660-666

Eap CB, Buclin T, Baumann P (2002) Interindividual variability of the clinical pharmacokinetics of methadone Clinical pharmacokinetics 41:1153-1193

Eap CB et al. (2007) Stereoselective block of hERG channel by (S)-methadone and QT interval prolongation in CYP2B6 slow metabolizers Clinical Pharmacology \& Therapeutics 81:719-728

Eap CB, Cuendet C, Baumann P (1990) Binding of d-methadone, 1-methadone, and dl-methadone to proteins in plasma of healthy volunteers: Role of the variants of $\alpha 1$-acid glycoprotein Clinical Pharmacology \& Therapeutics 47:338-346

European Food Safety Authority (EFSA) (2017) Update: use of the benchmark dose approach in risk assessment EFSA Journal 15:e04658

Ehret GB et al. (2006) Drug-induced long QT syndrome in injection drug users receiving methadone: high frequency in hospitalized patients and risk factors Archives of internal medicine 166:1280-1287 
Esses JL, Rosman J, Do LT, Schweitzer P, Hanon S (2008) Successful transition to buprenorphine in a patient with methadone-induced torsades de pointes Journal of interventional cardiac electrophysiology 23:117119

Ewart L et al. (2014) The concordance between nonclinical and phase I clinical cardiovascular assessment from a cross-company data sharing initiative Toxicological Sciences 142:427-435

Ewart L et al. (2012) How do the top 12 pharmaceutical companies operate safety pharmacology? Journal of pharmacological and toxicological methods 66:66-70

Fareed A, Vayalapalli S, Scheinberg K, Gale R, Casarella J, Drexler K (2013) QTc interval prolongation for patients in methadone maintenance treatment: a five years follow-up study The American journal of drug and alcohol abuse 39:235-240

Florian J, Garnett C, Nallani S, Rappaport B, Throckmorton D (2012) A modeling and simulation approach to characterize methadone QT prolongation using pooled data from five clinical trials in MMT patients Clinical Pharmacology \& Therapeutics 91:666-672

Foster DJ, Somogyi AA, Dyer KR, White JM, Bochner F (2000) Steady-state pharmacokinetics of (R)-and (S)methadone in methadone maintenance patients British journal of clinical pharmacology 50:427-440

Foster DJ, Somogyi AA, White JM, Bochner F (2004) Population pharmacokinetics of (R)-,(S)-and racmethadone in methadone maintenance patients British journal of clinical pharmacology 57:742-755

Foster DJ (2001) An examination of the metabolism and pharmacokinetics of methadone with respect to stereoselectivity. Dissertation, The Universtiy of Adelaide.

Fredheim OMS, Borchgrevink PC, Hegrenæs L, Kaasa S, Dale O, Klepstad P (2006) Opioid switching from morphine to methadone causes a minor but not clinically significant increase in QTc time: A prospective 9-month follow-up study Journal of pain and symptom management 32:180-185

Garg P, Garg V, Shrestha R, Sanguinetti MC, Kamp TJ, Wu JC (2018) Human induced pluripotent stem cellderived cardiomyocytes as models for cardiac channelopathies: a primer for non-electrophysiologists Circulation research 123:224-243

Garrido M, Aguirre C, Troconiz I, Marot M, Valle M, Zamacona M, Calvo R (2000) Alpha 1-acid glycoprotein (AAG) and serum protein binding of methadone in heroin addicts with abstinence syndrome International journal of clinical pharmacology and therapeutics 38:35-40

Gerber JG et al. (2001) Effect of ritonavir/saquinavir on stereoselective pharmacokinetics of methadone: results of AIDS Clinical Trials Group (ACTG) 401 Journal of acquired immune deficiency syndromes (1999) 27:153-160

Harris K, Aylott M, Cui Y, Louttit JB, McMahon NC, Sridhar A (2013) Comparison of electrophysiological data from human-induced pluripotent stem cell-derived cardiomyocytes to functional preclinical safety assays toxicological sciences 134:412-426

Heesch CB, Copfer AE, Davis SJ, Edwards BW (2015) Evaluation of Methadone-Induced QTc Prolongation in a Veteran Population Federal Practitioner 32:36

Hsu Y-C et al. (2013) Methadone concentrations in blood, plasma, and oral fluid determined by isotope-dilution gas chromatography-mass spectrometry Analytical and bioanalytical chemistry 405:3921-3928

Huang Z, Ung T (2013) Effect of alpha-1-acid glycoprotein binding on pharmacokinetics and pharmacodynamics Current drug metabolism 14:226-238

The International Council for Harmonisation of Technical Requirements for Pharmaceuticals for Human Use (ICH) (2005a). S7B: The non-clinical evaluation of the potential for delayed ventricular re- polarization 
(QT interval prolongation) by human phaceuticals. https://database.ich.org/sites/default/files/S7B_Guideline.pdf. Accessed 20 November 2019

The International Council for Harmonisation of Technical Requirements for Pharmaceuticals for Human Use (ICH) (2005b). E14: The clinical evaluation of QT/QTc interval prolongation and proarrhythmic potential for non-antiarrhythmic drugs. https://database.ich.org/sites/default/files/E14_Guideline.pdf. Accessed 20 November 2019

Judson R et al. (2014) In vitro and modelling approaches to risk assessment from the US Environmental Protection Agency ToxCast programme Basic \& clinical pharmacology \& toxicology 115:69-76

Justo D, Gal-Oz A, Paran Y, Goldin Y, Zeltser D (2006) Methadone-associated Torsades de Pointes (polymorphic ventricular tachycardia) in opioid-dependent patients Addiction 101:1333-1338

Kannankeril P, Roden DM, Darbar D (2010) Drug-induced long QT syndrome Pharmacological reviews 62:760781

Katchman AN, McGroary KA, Kilborn MJ, Kornick CA, Manfredi PL, Woosley RL, Ebert SN (2002) Influence of opioid agonists on cardiac humanether-a-go-go-related gene $\mathrm{K}+$ currents Journal of Pharmacology and Experimental Therapeutics 303:688-694

Ke AB, Nallani SC, Zhao P, Rostami-Hodjegan A, Unadkat JD (2014) Expansion of a PBPK model to predict disposition in pregnant women of drugs cleared via multiple CYP enzymes, including CYP2B6, CYP2C9 and CYP2C19 British journal of clinical pharmacology 77:554-570

Kharasch ED (2017) Current concepts in methadone metabolism and transport Clinical pharmacology in drug development 6:125-134

Kharasch ED, Hoffer C, Whittington D, Sheffels P (2004) Role of hepatic and intestinal cytochrome P450 3A and 2B6 in the metabolism, disposition, and miotic effects of methadone Clinical Pharmacology \& Therapeutics 76:250-269

Kharasch ED, Walker A, Whittington D, Hoffer C, Bedynek PS (2009) Methadone metabolism and clearance are induced by nelfinavir despite inhibition of cytochrome P4503A (CYP3A) activity Drug and alcohol dependence 101:158-168

Kitaguchi T et al. (2017) CSAHi study: detection of drug-induced ion channel/receptor responses, QT prolongation, and arrhythmia using multi-electrode arrays in combination with human induced pluripotent stem cell-derived cardiomyocytes Journal of pharmacological and toxicological methods $85: 73-81$

Krantz MJ, Lewkowiez L, Hays H, Woodroffe MA, Robertson AD, Mehler PS (2002) Torsade de pointes associated with very-high-dose methadone Annals of internal medicine 137:501-504

Krantz MJ, Lowery CM, Martell BA, Gourevitch MN, Arnsten JH (2005) Effects of methadone on QT-interval dispersion Pharmacotherapy: The Journal of Human Pharmacology and Drug Therapy 25:1523-1529

Kratz JM, Grienke U, Scheel O, Mann SA, Rollinger JM (2017) Natural products modulating the hERG channel: heartaches and hope Natural product reports 34:957-980

Kuryshev YA, Kirsch GE, Brown AM (2010) Increased cardiac risk in concomitant methadone and diazepam treatment: pharmacodynamic interactions in cardiac ion channels Biophysical Journal 98:339a

Li H, Zhang M, Vervoort J, Rietjens IMCM, van Ravenzwaay B, Louisse J (2017) Use of physiologically based kinetic modeling-facilitated reverse dosimetry of in vitro toxicity data for prediction of in vivo developmental toxicity of tebuconazole in rats Toxicology letters 266:85-93 
Li X, Zhang R, Zhao B, Lossin C, Cao Z (2016) Cardiotoxicity screening: a review of rapid-throughput in vitro approaches Archives of toxicology 90:1803-1816

Lin C, Somberg T, Molnar J, Somberg J (2009). The effects of chiral isolates of methadone on the cardiac potassium channel IKr. Cardiology, 113(1), 59-65.

Liu P, Foster G, LaBadie R, Somoza E, Sharma A (2007) Pharmacokinetic interaction between voriconazole and methadone at steady state in patients on methadone therapy Antimicrobial agents and chemotherapy 51:110-118

Louisse J, Beekmann K, Rietjens IMCM (2017) Use of physiologically based kinetic modeling-based reverse dosimetry to predict in vivo toxicity from in vitro data Chemical research in toxicology 30:114-125

Louisse J et al. (2010) The use of in vitro toxicity data and physiologically based kinetic modeling to predict doseresponse curves for in vivo developmental toxicity of glycol ethers in rat and man Toxicological Sciences 118:470-484

Lugo RA, Satterfield KL, Kern SE (2005) Pharmacokinetics of methadone Journal of pain \& palliative care pharmacotherapy 19:13-24

Ma J et al. (2011) High purity human-induced pluripotent stem cell-derived cardiomyocytes: electrophysiological properties of action potentials and ionic currents American Journal of Physiology-Heart and Circulatory Physiology 301:H2006-H2017

Maremmani I, Pacini M, Cesaroni C, Lovrecic M, Perugi G, Tagliamonte A (2005) QTc interval prolongation in patients on long-term methadone maintenance therapy European addiction research 11:44-49

Martell BA, Arnsten JH, Krantz MJ, Gourevitch MN (2005) Impact of methadone treatment on cardiac repolarization and conduction in opioid users The American journal of cardiology 95:915-918

Martin RL, McDermott JS, Salmen HJ, Palmatier J, Cox BF, Gintant GA (2004) The utility of hERG and repolarization assays in evaluating delayed cardiac repolarization: influence of multi-channel block Journal of cardiovascular pharmacology 43:369-379

Mirams GR et al. (2011) Simulation of multiple ion channel block provides improved early prediction of compounds' clinical torsadogenic risk Cardiovascular research 91:53-61

Mishra H, Polak S, Jamei M, Rostami-Hodjegan A (2014) Interaction between domperidone and ketoconazole: toward prediction of consequent QTc prolongation using purely in vitro information CPT: pharmacometrics \& systems pharmacology 3:1-11

Moody DE, Lin S-N, Chang Y, Lamm L, Greenwald MK, Ahmed MS (2008) An enantiomer-selective liquid chromatography-tandem mass spectrometry method for methadone and EDDP validated for use in human plasma, urine, and liver microsomes Journal of analytical toxicology 32:208-219

Mujtaba S, Romero J, Taub CC (2013) Methadone, QTc prolongation and torsades de pointes: current concepts, management and a hidden twist in the tale? Journal of cardiovascular disease research 4:229-235

Nakamura Y et al. (2014) Assessment of testing methods for drug-induced repolarization delay and arrhythmias in an iPS cell-derived cardiomyocyte sheet: Multi-site validation study Journal of pharmacological sciences: $13248 \mathrm{FP}$

Nilsson M-I, Meresaar U, ÄNggård E (1982) Clinical pharmacokinetics of methadone Acta Anaesthesiologica Scandinavica 26:66-69

Ning J, Louisse J, Spenkelink B, Wesseling S, Rietjens IMCM (2017) Study on inter-ethnic human differences in bioactivation and detoxification of estragole using physiologically based kinetic modeling Archives of toxicology 91:3093-3108 
Nozaki Y et al (2017) CSAHi study-2: validation of multi-electrode array systems (MEA60/2100) for prediction of drug-induced proarrhythmia using human iPS cell-derived cardiomyocytes: assessment of reference compounds and comparison with nonclinical studies and clinical information. Regul Toxicol Pharmacol $88: 238-251$

Oda Y, Kharasch ED (2001) Metabolism of Methadone andlevo- $\alpha$-Acetylmethadol (LAAM) by Human Intestinal Cytochrome P450 3A4 (CYP3A4): Potential Contribution of Intestinal Metabolism to Presystemic Clearance and Bioactivation Journal of Pharmacology and Experimental Therapeutics 298:1021-1032

Olsen GD (1973) Methadone binding to human plasma proteins Clinical Pharmacology \& Therapeutics 14:338343

Pang L et al. (2019) Workshop Report: FDA Workshop on Improving Cardiotoxicity Assessment With HumanRelevant Platforms Circulation research 125:855-867

Peles E, Bodner G, Kreek MJ, Rados V, Adelson M (2007) Corrected-QT intervals as related to methadone dose and serum level in methadone maintenance treatment (MMT) patients - a cross-sectional study Addiction 102:289-300

Reddy S, Hui D, Osta BE, de la Cruz M, Walker P, Palmer JL, Bruera E (2010) The effect of oral methadone on the QTc interval in advanced cancer patients: a prospective pilot study Journal of palliative medicine $13: 33-38$

Redfern W et al. (2003) Relationships between preclinical cardiac electrophysiology, clinical QT interval prolongation and torsade de pointes for a broad range of drugs: evidence for a provisional safety margin in drug development Cardiovascular research 58:32-45

Rehnelt S et al. (2017) Frequency-dependent multi-well cardiotoxicity screening enabled by optogenetic stimulation International journal of molecular sciences 18:2634

Rietjens IMCM, Louisse J, Punt A (2011) Tutorial on physiologically based kinetic modeling in molecular nutrition and food research Molecular nutrition \& food research 55:941-956

Romach M, Piafsky K, Abel J, Khouw V, Sellers E (1981) Methadone binding to orosomucoid ( $\alpha 1$-acid glycoprotein): Determinant of free fraction in plasma Clinical Pharmacology \& Therapeutics 29:211217

Roy AK, McCarthy C, Kiernan G, McGorrian C, Keenan E, Mahon NG, Sweeney B (2012) Increased incidence of QT interval prolongation in a population receiving lower doses of methadone maintenance therapy Addiction 107:1132-1139

Sala L, Ward-van Oostwaard D, Tertoolen LG, Mummery CL, Bellin M (2017) Electrophysiological analysis of human pluripotent stem cell-derived cardiomyocytes (hPSC-CMs) using multi-electrode arrays (MEAs) JoVE (Journal of Visualized Experiments)123:e55587

Smith DA, Di L, Kerns EH (2010) The effect of plasma protein binding on in vivo efficacy: misconceptions in drug discovery Nature reviews Drug discovery 9:929-939

Stevens JL, Baker TK (2009) The future of drug safety testing: expanding the view and narrowing the focus Drug discovery today 14:162-167

Strikwold M, Spenkelink B, de Haan LH, Woutersen RA, Punt A, Rietjens IMCM (2017) Integrating in vitro data and physiologically based kinetic (PBK) modelling to assess the in vivo potential developmental toxicity of a series of phenols Archives of toxicology 91:2119-2133 
Strikwold M, Spenkelink B, Woutersen RA, Rietjens IMCM, Punt A (2013) Combining in vitro embryotoxicity data with physiologically based kinetic (PBK) modelling to define in vivo dose-response curves for developmental toxicity of phenol in rat and human Archives of toxicology 87:1709-1723

Stringer J, Welsh C, Tommasello A (2009) Methadone-associated QT interval prolongation and torsades de pointes American Journal of Health-System Pharmacy 66:825-833

Sullivan HR, Due SL (1973) Urinary metabolites of dl-methadone in maintenance subjects Journal of medicinal chemistry 16:909-913

Taguchi K, Nishi K, Chuang VTG, Maruyama T, Otagiri M (2013) Molecular aspects of human alpha-1 acid glycoprotein — structure and function. In: Janciauskiene S (ed) Acute phase proteins. InTech, Croatia, pp 139-162

Thermo Fisher Scientific (2017) User Guide: Single-Use RED Plate with Inserts. https://assets.thermofisher.com/TFS-

Assets/LSG/manuals/MAN0011619_SgleUse_RED_Plate_Insert_UG.pdf. Accessed 20 November 2019

Totah RA, Sheffels P, Roberts T, Whittington D, Thummel K, Kharasch ED (2008) Role of CYP2B6 in stereoselective human methadone metabolism Anesthesiology: The Journal of the American Society of Anesthesiologists 108:363-374

Treece JM et al. (2018) Comprehensive review on methadone-induced QT prolongation and torsades Journal of Pharmacology and Pharmacotherapeutics 9:66

van Liempd S, Morrison D, Sysmans L, Nelis P, Mortishire-Smith R (2011) Development and validation of a higher-throughput equilibrium dialysis assay for plasma protein binding JALA: Journal of the Association for Laboratory Automation 16:56-67

Vandenberk B et al. (2016) Which QT correction formulae to use for QT monitoring? Journal of the American Heart Association 5:e03264

Verebely K, Volavka J, Mulé S, Resnick R (1975) Methadone in man: pharmacokinetic and excretion studies in acute and chronic treatment Clinical Pharmacology \& Therapeutics 18:180-190

Wakefield ID, Pollard C, Redfern WS, Hammond TG, Valentin JP (2002) The application of in vitro methods to safety pharmacology Fundamental \& clinical pharmacology 16:209-218

Waters NJ, Jones R, Williams G, Sohal B (2008) Validation of a rapid equilibrium dialysis approach for the measurement of plasma protein binding Journal of pharmaceutical sciences 97:4586-4595

Wedam EF, Bigelow GE, Johnson RE, Nuzzo PA, Haigney MC (2007) QT-interval effects of methadone, levomethadyl, and buprenorphine in a randomized trial Archives of internal medicine 167:2469-2475

Wheeler MW, Bailer AJ (2007) Properties of model-averaged BMDLs: A study of model averaging in dichotomous response risk estimation Risk Analysis: An International Journal 27:659-670

World Health Organization (WHO). (2010). Characterization and application of physiologically based pharmacokinetic models in risk assessment. http://www.inchem.org/documents/harmproj/harmproj/harmproj9.pdf. Accessed 20 November 2019

Wilkins JN, Ashofteh A, Setoda D, Wheatley WS, Huigen H, Ling W (1997) Ultrafiltration using the Amicon MPS-1 for assessing methadone plasma protein binding Therapeutic drug monitoring 19:83-87

Wolff K, Rostami-Hodjegan A, Hay A, Raistrick D, Tucker G (2000) Population-based pharmacokinetic approach for methadone monitoring of opiate addicts: potential clinical utility Addiction 95:1771-1783 
Yang F, Tong X, McCarver DG, Hines RN, Beard DA (2006) Population-based analysis of methadone distribution and metabolism using an age-dependent physiologically based pharmacokinetic model Journal of pharmacokinetics and pharmacodynamics 33:485-518

Zhao S, Kamelia L, Boonpawa R, Wesseling S, Spenkelink B, Rietjens IMCM (2019) Physiologically Based Kinetic Modeling-Facilitated Reverse Dosimetry to Predict In Vivo Red Blood Cell Acetylcholinesterase Inhibition Following Exposure to Chlorpyrifos in the Caucasian and Chinese Population Toxicological Sciences 171:69-83

Zwartsen A, de Korte T, Nacken P, de Lange DW, Westerink RH, Hondebrink L (2019) Cardiotoxicity screening of illicit drugs and new psychoactive substances (NPS) in human iPSC-derived cardiomyocytes using microelectrode array (MEA) recordings Journal of molecular and cellular cardiology 136:102-112 


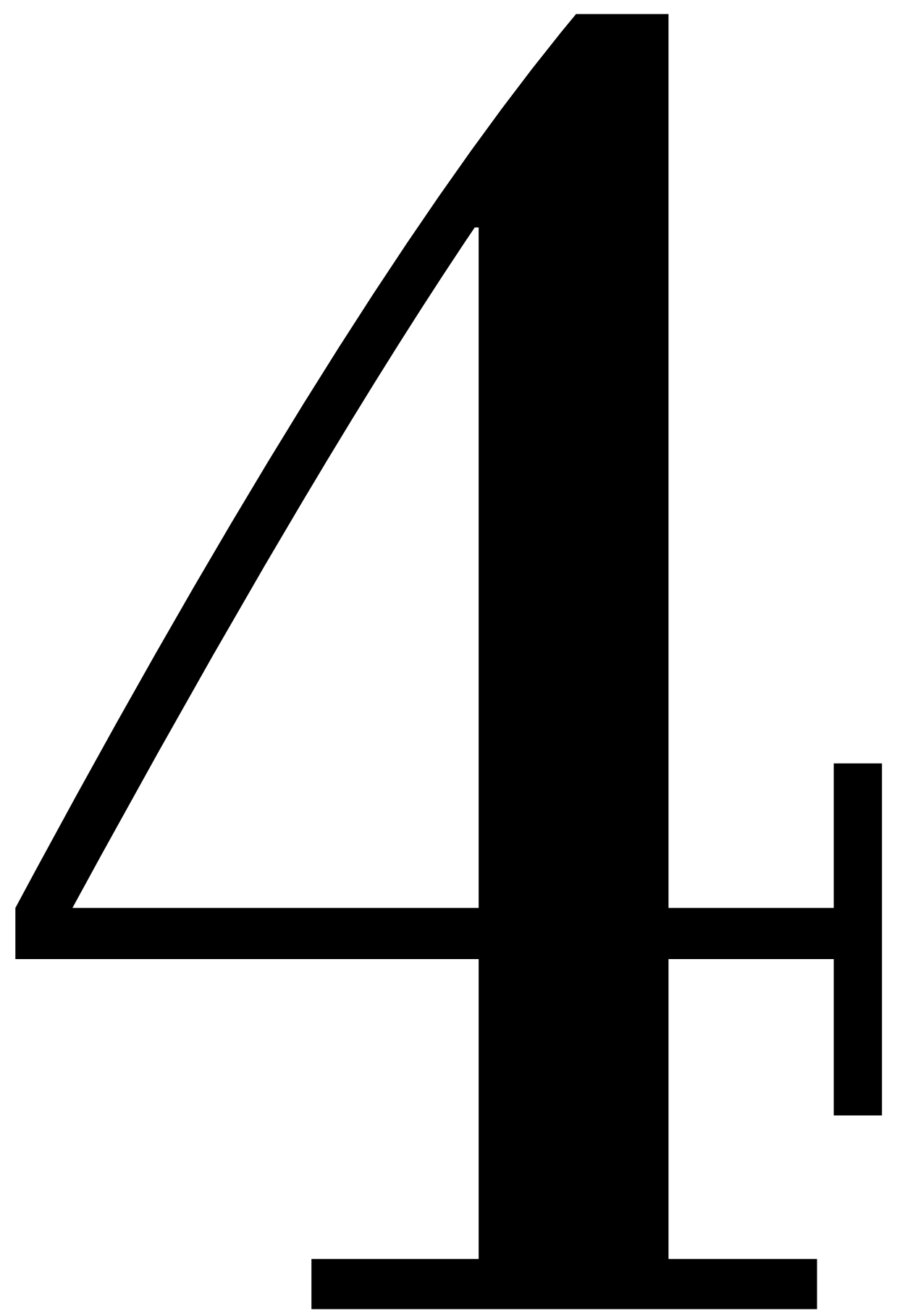




\section{Chapter 4}

\section{In vitro-in silico-based prediction of inter-individual and inter-ethnic variations in the dose-dependent cardiotoxicity of $R$ - and $S$ - methadone in humans}

Miaoying Shi, Yumeng Dong, Hans Bouwmeester, Ivonne M. C.

M. Rietjens, Marije Strikwold

Submitted 


\begin{abstract}
New approach methodologies predicting human cardiotoxicity are of interest to support or even replace in vivo-based drug safety testing. The present study presents an in vitro-in silico approach to predict the effect of inter-individual and inter-ethnic kinetic variations in the cardiotoxicity of R- and S-methadone in the Caucasian and the Chinese population. In vitro cardiotoxicity data, and metabolic data obtained from two approaches, using either individual human liver microsomes or recombinant cytochrome P450 enzymes (rCYPs), were integrated with physiologically based kinetic (PBK) models and Monte Carlo simulations to predict interindividual and inter-ethnic variations in methadone-induced cardiotoxicity. Chemical specific adjustment factors were defined and used to derive dose-response curves for the sensitive individuals. Our simulations indicated that Chinese are more sensitive towards methadoneinduced cardiotoxicity with Margin of Safety values being generally 2-fold lower than those for Caucasians for both methadone enantiomers. Individual PBK models using microsomes and PBK models using rCYPs combined with Monte Carlo simulations predicted similar interindividual and inter-ethnic variations in methadone-induced cardiotoxicity. The present study illustrates how inter-individual and inter-ethnic variations in cardiotoxicity can be predicted by combining in vitro toxicity and metabolic data, PBK modelling and Monte Carlo simulations. The novel methodology can be used to enhance cardiac safety evaluations of drugs in the preclinical stage and facilitate the design for dosing regiments in clinical trials.
\end{abstract}




\section{Introduction}

Cardiotoxicity is an important endpoint in drug safety evaluation as it has been a leading cause of drug attrition during the development stage and leads to the withdrawal of marketed drugs (Ferri et al., 2013). Recently we demonstrated that quantitative in vitro to in vivo extrapolation (QIVIVE) using physiologically based kinetic (PBK) modelling-based reverse dosimetry is an adequate approach to predict in vivo cardiotoxicity of racemic methadone (rac-methadone) in humans (Shi et al., 2020a). In this previous work, the cardiotoxic effects of rac-methadone on human induced pluripotent stem cell-derived cardiomyocytes (hiPSC-CMs) were quantified in vitro using the multi-electrode array (MEA) system. The obtained in vitro concentrationresponse curve for the field potential duration corrected for beat rate (FPDc), resembling the parameters observed in the human ECG (Zwartsen et al., 2019), was extrapolated to a predicted in vivo dose-response curve, which matched well with in vivo clinical data on rac-methadoneinduced QTc prolongation.

Methadone is a prescription drug for the treatment of opioid addiction and chronic pain, which however has been associated with QTc interval prolongation in the clinic (Alinejad et al., 2015). Methadone is usually administered as the racemic preparation, a 1:1 mixture of the R- and S-enantiomer, with mainly the S-enantiomers being responsible for the cardiotoxic effects observed in vivo (Ansermot et al., 2010) and in vitro (Eap et al., 2007). Eap et al. (2007), reported that S-methadone showed a 3.5-fold higher potency than R-methadone in blocking the human ether-à-go-go-related gene (hERG) currents which play an important role in cardiac repolarization (Martin et al., 2004). Methadone is predominately cleared by the hepatic cytochrome P450 (CYP) enzymes via $N$-demethylation and cyclisation to its primary metabolite 2-ethylidene-1,5-dimethyl-3,3-diphenylpyrrolidine (EDDP), which was not found to be cardiotoxic in vitro at therapeutic relevant internal concentrations (Eap et al., 2007; Shi et al., 2020a). The major enzymes mediating the formation of EDDP have been identified in both in vitro and in vivo studies to be CYP2B6, CYP3A4 and to a lesser extent CYP2C19, with CYP2C19 and CYP2B6 showing stereoselectivity towards the conversion of R- and Smethadone, while CYP3A4 appeared to convert R- and S- methadone without stereoselectivity (Chang et al., 2011; Eap et al., 2007; Foster et al., 1999; Gerber et al., 2004; Kharasch, 2017; Totah et al., 2007).

An increasing number of drug failures has been associated to unexpected extreme effects or inefficacy effects in clinical studies, pointing out the importance of studying inter-individual 
variation in response to drug candidates and identifying covariates resulting in such variations (Tracy et al., 2016). Ethnic differences in demography, physiology and genetic background may affect the kinetic processes thereby contributing to uncertainty in the safety evaluation of compounds (Malinowski et al., 2008; Ning et al., 2017). Moreover, polymorphisms in CYP enzymes is considered to be one of the most important factors contributing to the interindividual variability in sensitivity towards compound exposure and thus needs to be incorporated in deciding on individual dosing regimens (Chiba et al., 2017; Zanger and Schwab, 2013). The PBK model established in our previous work (Shi et al., 2020a) was defined for the Caucasian population as a whole while inter-individual and inter-ethnic differences in kinetics were not yet considered. Large inter-individual variations in methadone pharmacokinetics have been reported to be the result of variability in the expression of the CYP isoforms involved in methadone metabolism (Eap et al., 2002). Given the highly polymorphic gene of CYP2B6 (Kharasch, 2017; Zanger and Klein, 2013) and the large variations between Caucasians and Chinese in the abundance of CYP3A4 (Barter et al., 2013), it is of interest to include such variabilities in the PBK model-based reverse dosimetry approach and predict their effects on the in vivo cardiotoxicity of R- and S-methadone.

PBK modelling and Monte Carlo simulations have been used to assess inter-individual variation in drug safety evaluations (Ito et al., 2017; Mehrotra et al., 2012). However, most studies involve in vivo data and specific dose regimens while the inter-individual variation on the whole population level for different dose regimens was not quantitatively evaluated. In the safety assessment of chemicals, the International Programme on Chemical Safety (IPCS) has proposed the chemical-specific adjustment factor (CSAF) as a standard parameter to quantify inter-species or human inter-individual differences in toxicokinetics or toxicodynamics, while such a factor may equally well be defined for chemical drugs like methadone (IPCS, 2005). The default uncertainty factor of 10 is set for human inter-individual differences with a subdivision for a factor of 3.16 accounting for human variability in toxicokinetics and 3.16 for variability in toxicodynamics (IPCS, 2005).

Previously a new approach methodology (NAM, ICCVAM, 2018) combining in vitro data, PBK modelling and Monte Carlo simulations has been used to predict inter-individual and/or inter-ethnic variations in in vivo toxicity for developmental toxicity of phenol (Strikwold et al., 2017) and liver toxicity of lasiocarpine (Ning et al., 2019). The aim of the present study was to demonstrate such an approach for the cardiotoxicity of R- and S-methadone, and to elucidate the consequences of inter-ethnic and inter-individual kinetic variations for the sensitivity 
towards these pharmaceuticals. To obtain this insight, PBK models for the two methadone enantiomers were developed and variations in their CYP-mediated metabolism were incorporated using two different approaches including 1) metabolic variation obtained from incubations with 25 Caucasian and 25 Chinese individual human liver microsomes (HLMs), and 2) reported variation in CYP abundances combined with Monte Carlo simulations. Ultimately the maximum concentrations $\left(\mathrm{C}_{\max }\right)$ of $\mathrm{R}$ - and $\mathrm{S}$-methadone in the heart venous blood were predicted, from which CSAFs were derived to describe the inter-individual kinetic variations within the different populations. Subsequently the CSAFs were applied to the predicted in vivo dose-response curves obtained by reverse dosimetry of in vitro cardiotoxicity data to predict the toxicity for the most sensitive individuals within the populations based on which the safety in use of R- and S-methadone was discussed.

\section{Materials and methods}

\subsection{Chemical and biological materials}

Rac-methadone hydrochloride ( $\geq 98 \%$, R-methadone: S-methadone 1:1), rac-EDDP perchlorate ( $\geq 98 \%$, R-EDDP: S-EDDP $1: 1$ ), Tris (hydroxymethyl) aminomethane (Trizma ${ }^{\circledR}$ base), and ammonium acetate were purchased from Sigma-Aldrich (Zwijndrecht, The Netherlands). The use of rac-methadone was in compliance with the registration (opium exemption license number $10478303 \mathrm{WCO}$ ) at Farmatec (executive organization of the Ministry of Health, Welfare and Sport, The Hague, The Netherlands). Dimethyl sulfoxide (DMSO, 99.7\%) was obtained from Merck (Schiphol-Rijk, The Netherlands). Acetonitrile (ACN, UPLC/MS grade) was obtained from Biosolve BV (Valkenswaard, The Netherlands). Reduced nicotinamide adenine dinucleotide phosphate (NADPH) regenerating system solution A and solution B were purchased from Corning (Woburn, MA, USA). Twenty-five individual Caucasian male human liver microsomes were obtained from XenoTech (Lenexa, USA). Twenty-five individual Chinese male human liver microsomes were purchased from PrimeTox (Wuhan, China). Detailed information of the human liver microsome donors are shown in Table S1 in the supplementary materials 1 .

\subsection{General outline of PBK modelling and Monte Carlo simulation}

To investigate the inter-individual and inter-ethnic variations in the cardiotoxicity of R- and Smethadone, the present study included the following steps: (1) Generation of information on the metabolic variation in CYP-mediated conversion of R- and S-methadone using two approaches. In the first approach information on the metabolic variation of R-and S-methadone 
conversion was generated from in vitro kinetic experiments using 25 Chinese and 25 Caucasian individual liver microsomes while in the second approach information on variation in the metabolism was obtained based on reported kinetic constants for R- and S-methadone of recombinant CYP isoforms (rCYPs) together with reported variation in their expression in the Caucasian and the Chinese population. (2) Development and evaluation of PBK models for Rand S-methadone using the metabolic parameters obtained from the two approaches. (3) Integrating metabolic variations, PBK modelling and the Monte Carlo simulation to predict inter-individual and inter-ethnic variations in the kinetics of R- and S-methadone and the calculation of CSAFs for human kinetics. (4) PBK modelling-based reverse dosimetry and dose-response analysis of R- and S-methadone-induced cardiotoxicity for the average and the sensitive populations in the Caucasian and Chinese populations for the safety evaluation of Rand S-methadone.

\subsection{Generation of metabolic variation data in the conversion of $R$ - and $S-$ methadone toward R- and S-EDDP}

\subsubsection{In vitro incubations with 25 Caucasian and 25 Chinese individual liver microsomes}

In vitro incubations with 25 male Caucasian and 25 male Chinese individual liver microsomes were performed as previously described by Shi et al. (2020a). Based on the IPCS guideline this number of individual microsomes is sufficient to accurately measure the central tendency of the whole population (IPCS 2005). In brief, incubation samples with a final volume of $160 \mu 1$ were prepared in $0.1 \mathrm{M}$ Tris- $\mathrm{HCl}$ ( $\mathrm{pH} 7.4$ ) containing the NADPH regeneration system (final concentrations $1.3 \mathrm{mM} \mathrm{NADP}{ }^{+}, 3.3 \mathrm{mM}$ glucose-6-phosphate, $0.4 \mathrm{U} / \mathrm{ml}$ glucose-6-phosphate dehydrogenase and $3.3 \mathrm{mM}$ magnesium chloride) and rac-methadone at seven final concentrations ranging from 25 to $1500 \mu \mathrm{M}$ added from a concentrated stock solution of 100 $\mathrm{mM}$ in water. Control samples were prepared in the same way, but in the absence of NAPDH regeneration system which was replaced with Tris-HCl. Samples were pre-incubated at $37^{\circ} \mathrm{C}$ for one minute and the reactions were started by adding individual human liver microsomes at a final concentration of $0.5 \mathrm{mg} / \mathrm{ml}$ microsomal protein. After $40 \mathrm{~min}$ incubation at $37{ }^{\circ} \mathrm{C}, 40$ $\mu 1$ ice-cold ACN were added to terminate the reaction. Then samples were put on ice for 20 min and centrifuged at $18,000 \mathrm{~g}$ for $5 \mathrm{~min}$ at $4{ }^{\circ} \mathrm{C}$ to precipitate microsomal proteins. The supernatant was collected and diluted 2 to 10 times with ACN for the quantification of R- and S-EDDP by liquid chromatography-mass spectrometry (LC-MS/MS) as described in the "LCMS/MS analysis" section. Under these conditions the reaction rate was shown to be linear with 
respect to incubation time and microsomal protein concentration. Given that no gender differences in metabolism of methadone have been reported in the literature (Graziani and Nisticò, 2015) and that the average of catalytic efficiency for rac-methadone metabolism obtained from 25 male Caucasian HLMs was comparable with the one obtained from the mixgender microsomal pool of 150 donors (Shi et al., 2020a), the metabolic variations derived from male individuals are expected to be comparable those for mixed gender.

The metabolic parameters including the apparent maximum reaction rate $\left(\mathrm{V}_{\max }\right)$ and the apparent Michaelis-Menten constant $\left(\mathrm{K}_{\mathrm{m}}\right)$ for the formation of R- and S-EDDP were defined using GraphPad Prism 5.0 (GraphPad Software Inc., San Diego, USA.) to fit the data obtained from the in vitro microsomal incubations to the Michaelis-Menten equation (1):

$$
\mathrm{V}=\frac{\mathrm{V}_{\max } *[\mathrm{~S}]}{\mathrm{K}_{\mathrm{m}}+[\mathrm{S}]}
$$

where $[\mathrm{S}]$ is the substrate concentration $(\mu \mathrm{M})$ and $\mathrm{v}$ is the rate of R-and S-EDDP formation (nmol/min/mg microsomal protein). The in vitro catalytic efficiency expressed in $\mu 1 / \mathrm{min} / \mathrm{mg}$ microsomal protein was calculated by dividing $\mathrm{V}_{\max }$ by $\mathrm{K}_{\mathrm{m}}$. Data were collected from 2 independent experiments and each data point is presented as the mean value $\pm \mathrm{SD}$. The mean values and the coefficient of variations $(\mathrm{CVs})$ of $\mathrm{V}_{\max }$ and $\mathrm{K}_{\mathrm{m}}$ were calculated using Microsoft Excel 2016 (Microsoft Corporation, Washington, USA).

\subsubsection{Kinetic constants for $R$ - and S-methadone conversion by rCYPs and variations in CYP abundances in the Caucasian and the Chinese population}

CYP3A4, CYP2B6 and CYP2C19 are the major CYPs involved in the metabolism of both methadone enantiomers (Chang et al., 2011; Totah et al., 2007), and their kinetic constants ( $\mathrm{V}_{\max , \text { CYP }}$ and $\mathrm{K}_{\mathrm{m}, \mathrm{CYP}}$ ) for the conversion of R- and S-methadone toward R- and S-EDDP were obtained from the study of Totah et al. (2007). These kinetic constants were determined using Baculovirus-insect cells (Supersomes) expressing recombinant CYP2B6, CYP2C19 and CYP3A4 and the kinetic constants for methadone conversion by each CYP are shown in Table 1. To correct for the differences between the activity of the CYPs in the rCYP system and the HLM system, the reported $V_{\max }$ for Supersomes $\left(\mathrm{V}_{\max , \mathrm{CYP}}, \mathrm{pmol} / \mathrm{min} / \mathrm{pmol} \mathrm{CYP}\right)$ were scaled to the $\mathrm{V}_{\max }$ value for microsomes $\left(\mathrm{V}_{\max }\right.$ CYP in HLM, $\mathrm{pmol} / \mathrm{min} / \mathrm{mg}$ protein) using the following equation (2):

$$
\mathrm{V}_{\text {max }} \text { CYP in } \mathrm{HLM}=\mathrm{V}_{\max }, \mathrm{CYP} * \mathrm{ISEF} * \mathrm{CYP} \text { abundance }
$$


where ISEF is the CYP isoform specific inter-system extrapolation factor to correct for differences in the intrinsic activity between Supersomes and microsomes taking into account the relative abundance of the respective CYP in HLM (Proctor et al., 2004). CYP abundance ( $\mathrm{pmol} / \mathrm{mg}$ protein) is the expression level of the individual CYP present in HLM samples which were collected from the literature (Table 1). The ISEFs for the three CYPs were calculated using the following equation (3) for each methadone enantiomer:

$$
\mathrm{ISEF}=\frac{\mathrm{CL} \text { int, CYP in HLM }}{\mathrm{CL}_{\text {int, CYP }}{ }^{\mathrm{C}} \mathrm{CYP} \text { abundance }}
$$

where $\mathrm{CL}_{\text {int, }}$ CYP in HLM $(\mu \mathrm{l} / \mathrm{min} / \mathrm{mg}$ protein) represents in vitro intrinsic clearance of R- or Smethadone by each CYP in HLM, which were determined by multiplying the in vitro total CYP-mediated intrinsic clearance for the respective methadone enantiomer in Caucasian or Chinese HLM (CLint, HLM) measured in this study by the relative contribution of the respective CYP to the total CYPs in the HLM ( $\left.f_{m}, C Y P\right)$. This relative contribution, defined as fraction metabolised by each CYP of the total in vitro metabolic clearance amounted to 0.44, 0.09 and 0.46 (R-methadone), and 0.59, 0.09 and 0.32 (S-methadone) for CYP2B6, CYP2C19 and CYP3A4, respectively (Totah et al., 2008). These $f_{m}$, CYP values were obtained by the incubation of Caucasian HLM with inhibitors of the specific CYP isoforms (Totah et al., 2008). $\mathrm{CL}_{\text {int, CYP }}(\mu 1 / \mathrm{min} / \mathrm{pmol} \mathrm{CYP})$ represents the in vitro intrinsic clearance of the methadone

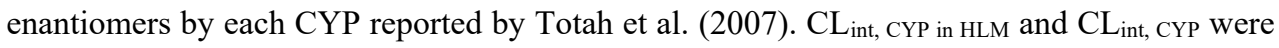
calculated from the enzyme kinetic parameters $\left(\mathrm{V}_{\max } / \mathrm{K}_{\mathrm{m}}\right)$ determined in HLM and the Supersomes, respectively. The calculated ISEFs for the Caucasians and Chinese are shown in Table 1. Due to lacking information about the $f_{m}$, CYP for the Chinese population, the $f_{m}$, CYP of the Caucasian was used to calculate ISEF values for the Chinese population. The detailed information used for calculation of the ISEF and $\mathrm{f}_{\mathrm{m}, \mathrm{CYP}}$ can be found in Table $\mathrm{S} 2$ and $\mathrm{S} 3$ in the supplementary materials 1 . 


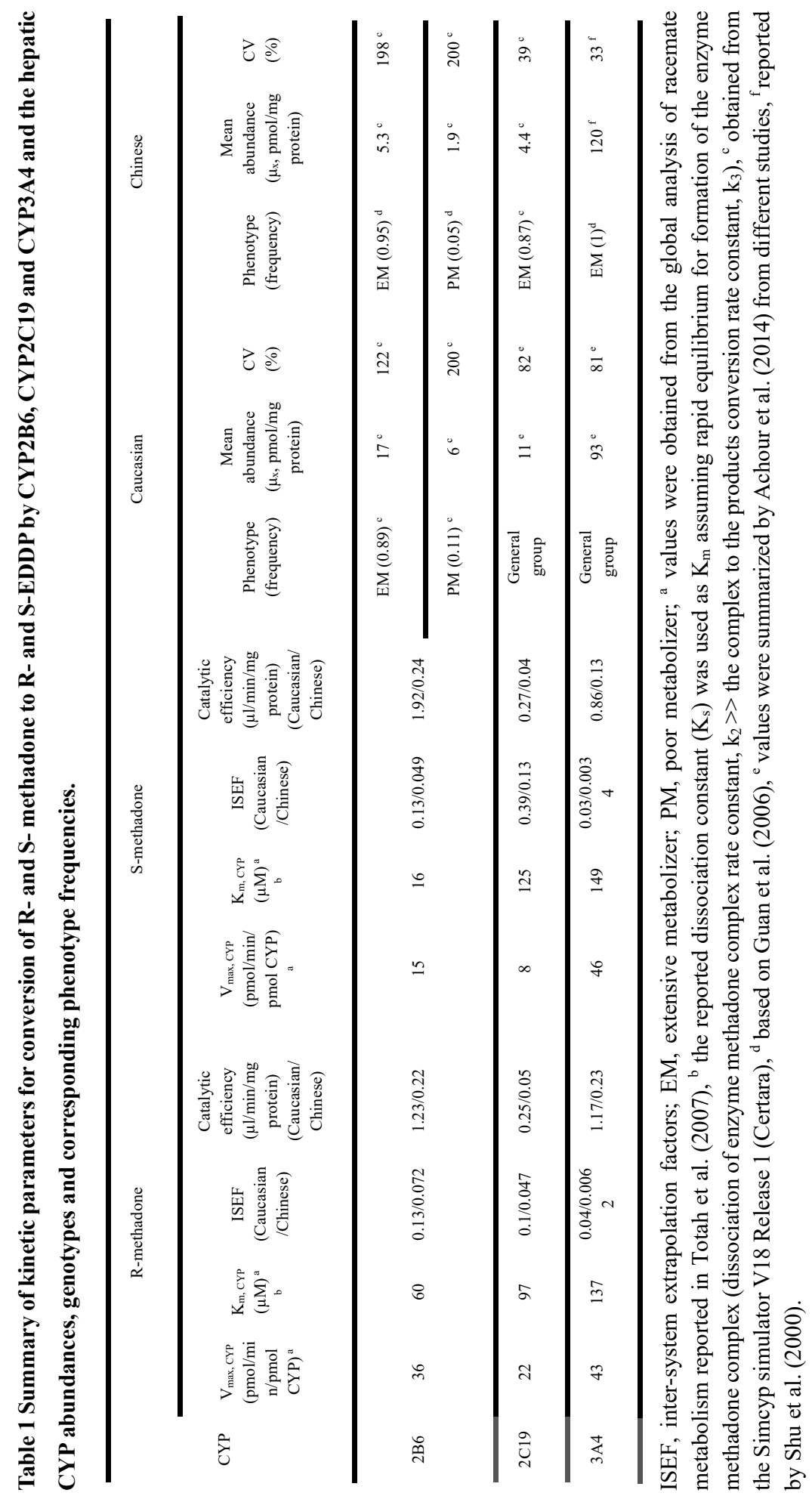




\subsection{LC-MS/MS analysis}

The chiral separation of R- and S-methadone and their metabolites and quantification was performed by LC-MS/MS analysis using a Shimadzu Nexera XR LC-20AD SR UPLC system coupled with a Shimadzu LCMS-8045 mass spectrometer (Kyoto, Japan). Samples were loaded on a CHIRALPAK ${ }^{\circledR}$ AGP column (100 x 4mm $5 \mu \mathrm{m}$ Analytical Column M) and CHIRALPAK $^{\circledR}$ AGP pre-column $(0.4 \mathrm{~cm} \times 1 \mathrm{~cm} 5 \mu \mathrm{m})$ with an injection volume of $1 \mu \mathrm{l}$. A Shimadzu LCMS-8045 triple quadrupole with electrospray ionization (ESI) interface was used to perform the MS-MS analysis. The instrument was operated in positive mode in the multiple reaction monitoring (MRM, N2 collision gas) mode. The multiple reaction monitoring of $\mathrm{m} / \mathrm{z}$ $310.20\left(\mathrm{MH}^{+}\right)$to $265.15(\mathrm{CE}:-15 \mathrm{kV}), \mathrm{m} / \mathrm{z} 310.20\left(\mathrm{MH}^{+}\right)$to $105.05(\mathrm{CE}:-28 \mathrm{kV})$ and m/z $310.20\left(\mathrm{MH}^{+}\right)$to $77.15(\mathrm{CE}:-51 \mathrm{kV})$ were used to analyse R- and S- methadone. The $\mathrm{m} / \mathrm{z}$ $278.10\left(\mathrm{MH}^{+}\right)$to $234.20(\mathrm{CE}:-31 \mathrm{kV}), \mathrm{m} / \mathrm{z} 278.10\left(\mathrm{MH}^{+}\right)$to $249.15(\mathrm{CE}:-25 \mathrm{kV})$ and m/z $278.10\left(\mathrm{MH}^{+}\right)$to $186.15(\mathrm{CE}:-38 \mathrm{kV})$ were used to analyse R- and S-EDDP. The MRMs were selected based on previous studies (Moody et al., 2008; Chang et al., 2011). For optimal chiral separation, an isocratic mobile phase of $10 \mathrm{mM}$ ammonium acetate $(\mathrm{pH}=7.0)$ : ACN (85: 15, $\mathrm{v} / \mathrm{v}$ ) with a flow rate of $1 \mathrm{ml} / \mathrm{min}$ was applied. The temperature of the column was kept at $20^{\circ} \mathrm{C}$. The retention times for R- EDDP, S-EDDP, R-methadone and S-methadone were 10.1, 12.9, 14.5 and $19.6 \mathrm{~min}$, respectively, determined using commercially available reference compounds. Quantification was based on comparison of the respective peak areas of the total ion chromatogram (TIC) to the TIC peak areas of corresponding linear calibration curves obtained from standards prepared in ACN using the reference compounds $\left(\mathrm{R}^{2}>0.999\right)$, using Postrun analysis in the software LabSolution (Shimadzu).

\subsection{Development of the PBK models of $R$ - and S-methadone for the Caucasian and Chinese population}

The PBK model of rac-methadone developed in the study of Shi et al. (2020a) was adjusted to describe the ADME of R- and S-methadone in the Caucasian and Chinese populations in the Berkeley Madonna software (version 8.3.18, UC Berkeley, CA, USA) applying Rosenbrock's algorithms for solving stiff systems. Figure 1 presents the schematic diagram of the PBK model and the compartments relevant for the ADME characteristics. The PBK model is developed for repeated dosing given that methadone is usually administrated daily.

Human physiological parameters used in the PBK model for the Caucasian population were obtained from Brown (Brown, Delp, Lindstedt, Rhomberg \& Beliles, 1997) et al. (1997) and 
for the Chinese population from NHFPC (2007a,b, 2014) (Table S4 in supplementary materials 1). The volume of the arterial, venous blood and the blood flow to the heart are not available for the Chinese population and were assumed to be the same as the ones for the Caucasian, which is regarded suitable since these parameters are not influential on the model outcome (see the results in sensitivity analysis). The physicochemical parameters of R- and S-methadone are presented in Table S5 in supplementary materials 1. Given that no chiral difference was reported in absorption related parameters (Ke et al., 2014; Badhan et al., 2019), values for these parameters were similar for both enantiomers, including a mean oral absorption rate constant (ka) value of 0.59 and a mean fraction absorbed $(\mathrm{Fa})$ value of 0.88 obtained from studies on rac-methadone (Foster et al., 2000; Ke et al., 2014).

Tissue: blood partition coefficients (P) of R- and S-methadone were obtained by dividing tissue: plasma partition coefficients by the corresponding blood/plasma ratio (BPr) to correct for the differences in the distribution of the compounds in blood and plasma. The BPr value of 0.7 reported by Hsu et al. (2013) was used and assumed to be similar for the two enantiomers (Badhan et al., 2019). The tissue: plasma partition coefficients of the two enantiomers (Table S5) were estimated using a QIVIVE tool (version 1.0) from Wageningen Food Safety Research (WFSR, 2020). The fraction unbound in plasma $\left(f_{u, p}\right)$, lipophilicity $(\log P)$ and acid-base properties (pKa) were used as the input for the algorithms of Berezhkovskiy (2004). The two enantiomers have the same $\log \mathrm{P}$ and $\mathrm{pKa}$ values, amounting to 3.93 and 9.2, respectively (Ke et al., 2014; Gerber et al., 2001). The mean $f_{u, p}$ values were obtained from several studies, amounting to 0.16 for R-methadone and 0.12 for S-methadone as reported by Ke et al. (2014).

As described in our validated PBK model for rac-methadone (Shi et al., 2020a), liver was considered as the metabolizing organ. The average values of kinetic constants $\left(\mathrm{V}_{\max }\right.$ and $\left.\mathrm{K}_{\mathrm{m}}\right)$ obtained from incubations with ethnic-specific individual microsomes were used to define the metabolism of R- and S-methadone in the two populations, applying Michaelis-Menten kinetics. Besides, the metabolism of R-and S-methadone at the microsomal level was defined by using reported rCYPs kinetic data of CYP2B6, CYP2C19 and CYP3A4. The enantiomeric interactions observed in the racemate metabolism using in vitro incubation of rCYPs (Totah et al., 2007) were included in the current model where the algorithms for the rate of R- and SEDDP formation was described by two-substrate, two-site models with the competitive inhibition enabling the homotropic and heterotropic binding. The algorithms were taken from equations reported in Totah et al. (2007) in which enantiomeric interactions were described for the CYP2B6, CYP2C19 and CYP3A4 separately (model equations are shown in 
supplementary materials 2). Predicted blood kinetics were not distinctive between enantiomeric interaction equations and Michaelis-Menten equation when both equations were modelled in the rCYP-based PBK model (data are not shown, both model equations are shown in supplementary materials 2), suggesting that predictions with the HLM PBK model without interaction are valid as well. The in vitro $\mathrm{V}_{\max }$ values were scaled to the in vivo situation by using a microsomal protein per gram of liver (MPPGL) value of $32 \mathrm{mg} / \mathrm{g}$ for the Caucasian population (Barter et al., 2007) and a value of $39.46 \mathrm{mg} / \mathrm{g}$ for the Chinese population (Zhang et al., 2015b).

Besides metabolism, urinary excretion significantly contributes to the elimination of methadone (Lugo et al., 2005), and thus the urinary excretion was included in the model. The renal clearance values (RCL) were set at $1.8 \mathrm{l} / \mathrm{h}$ for R-methadone and 1.1 for S-methadone as reported in the study of Ke et al. (2014).

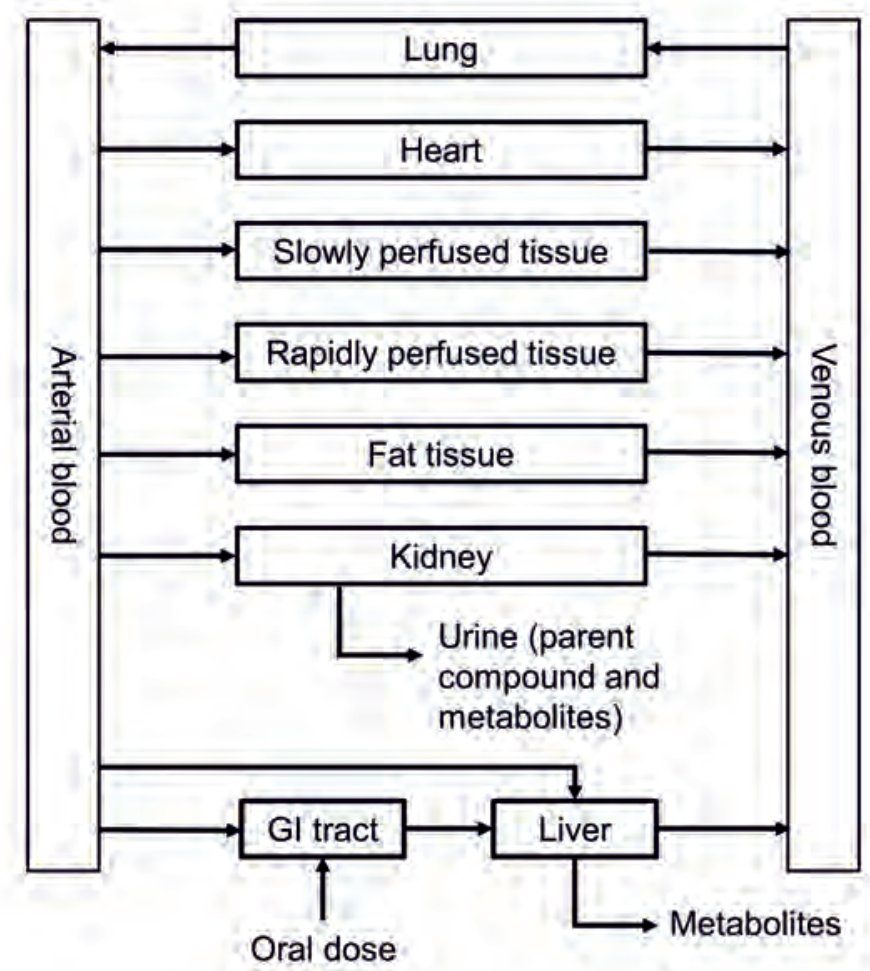

Figure 1 Schematic diagram of the PBK model of R- and S-methadone. 


\subsection{Sensitivity analysis and evaluation of the PBK model for R- and S- methadone}

The sensitivity analysis and model evaluation were performed for the PBK model with the average of the $\mathrm{V}_{\max }$ and $\mathrm{K}_{\mathrm{m}}$ values of the 25 individual HLMs and the PBK model with rCYPs defined metabolism data.

The influence of model parameters on the predicted R- and S-methadone $\mathrm{C}_{\max }$ in the heart venous blood during the steady-state phase was identified by performing a local parameter sensitivity analysis. The sensitivity coefficient (SC) was determined according to the following equation:

$$
\mathrm{SC}=\frac{\left(\mathrm{C}^{\prime}-\mathrm{C}\right)}{\left(\mathrm{P}^{\prime}-\mathrm{P}\right)} * \frac{\mathrm{P}}{\mathrm{C}}
$$

where $C$ represents the initial value of the model output, $C^{\prime}$ is the model output after a $1 \%$ increase in an individual model parameter value. Similarly, P stands for the initial parameter value and $\mathrm{P}^{\prime}$ is the parameter value after a $1 \%$ increase. Only parameters with an absolute SC > 0.1 are considered further, indicating a large impact on the model output (Rietjens et al., 2011). The sensitivity analysis was carried out for both the Caucasian and Chinese PBK model with the respective mean body weight of $70 \mathrm{~kg}$ (Brown et al., 1997) and $58.5 \mathrm{~kg}$ (NHFPC, 2007a), and for oral daily doses of 20 and $200 \mathrm{mg}$ rac-methadone (10 and $100 \mathrm{mg}$ of each enantiomers) for 30 days, as previously described (Shi et al., 2020a).

The performance of the developed PBK models for R- and S-methadone were evaluated by comparing the predicted blood concentrations and area under the curve (AUC) values of the enantiomers to the respective in vivo data on these parameters obtained in clinical studies (Foster et al., 2000; Garimella et al., 2015; Liu et al., 2007) where the time dependent concentrations of R- and S-methadone were measured in plasma. The reported plasma-based kinetics of R- and S-methadone were extracted using GetData Graph Digitizer 2.26 and converted to blood-based kinetics with multiplication by the respective BPr values. The specifications of in vivo studies are summarized in Table S6 and S7. Body weight, the oral dose and exposure duration were chosen to match the conditions used in the clinical studies for the PBK model evaluation. The performance of the enantiomeric PBK model for the Chinese was based on this evaluation of the model for the Caucasians since no in vivo kinetic data on dose-dependent blood or plasma levels of methadone enantiomers are available for the Chinese population. 


\subsection{Prediction of inter-individual and inter-ethnic variations applying individual PBK models and PBK modelling combined with Monte Carlo simulations}

The sensitivity analysis revealed that metabolic parameters are highly influential on the model output. To predict the influence of metabolic variations on the inter-individual and inter-ethnic differences in formation of R- and S-EDDP, two approaches were applied. In the first approach, 25 Caucasian and 25 Chinese individual PBK models were built by integrating the metabolic parameters obtained from the incubation of individual microsomes, to enable the prediction of $\mathrm{C}_{\max }$ in the heart venous blood of the two enantiomers at a clinically relevant daily dose of 30 $\mathrm{mg}$ of each enantiomer administered for 30 consecutive days for each individual applying a mean body weight of $70 \mathrm{~kg}$ for Caucasian and $58.5 \mathrm{~kg}$ for Chinese (Brown et al., 1997; NHFPC, 2007a).

In the second approach the Monte Carlo simulation was performed together with PBK modelling to simulate the variation in the $\mathrm{C}_{\max }$ in the heart venous blood of $\mathrm{R}$ - and $\mathrm{S}$-methadone in the Caucasian and the Chinese population. Monte Carlo simulations of the respective PBK models were run for each enantiomer in two populations with a daily dose of $30 \mathrm{mg}$ of each enantiomer administered for 30 days applying a mean body weight of $70 \mathrm{~kg}$ for Caucasian and $58.5 \mathrm{~kg}$ for Chinese (Brown et al., 1997; NHFPC, 2007a). To simulate the metabolic variation in R- and S-methadone formation, parameters for which random values were taken from the parameters' log-normal distribution for Monte Carlo simulation were the CYP abundances in the Caucasian and Chinese population. For the other parameters, fixed values were used. The distribution in the CYP abundances were defined by their mean value and CV in the Caucasian and Chinese population. For that purpose, lognormally distributed CYP abundances were transformed to a normally distributed variable $\omega$ with the mean $\left(\mu_{\omega}\right)$ and standard deviation $\left(\sigma_{\omega}\right)$ using the following equations (Zhang et al., 2007):

$$
\mu_{\omega}=\ln \left(\mu_{\mathrm{x}} / \sqrt{1+\mathrm{CV}_{\mathrm{x}}^{2}}\right)
$$

and

$$
\sigma_{\omega}^{2}=\ln \left(1+\mathrm{CV}_{\mathrm{x}}{ }^{2}\right)
$$


where $\mu_{\mathrm{x}}$ is the mean of CYPs abundances obtained from literature (Table 1). $C V_{\mathrm{x}}$ is the coefficient of variation of non-transformed CYP abundances. Monte Carlo simulations were performed in Berkeley Madonna (version 8.3.18, UC Berkeley) using the parameter plot function. Individuals with CYP abundances that were three times the standard derivation higher or lower than the mean values were excluded from the Monte Carlo simulation (Ning et al., 2019; Strikwold et al., 2017).

For the distribution of the CYP2B6 abundance in the Caucasian and the Chinese population two phenotypes were distinguished, namely the extensive metabolisers (EM) and the poor metabolizers (PM) using values reported in the Simcyp simulator V18 Release 1 (Certara, Sheffield, UK). For CYP2C19 and CYP3A4 the CYP abundance values for the general Caucasian population reported by Achour et al. (2014), without phenotype specification were used, while for the Chinese population the abundance distribution for the EM was used given the absence for the distribution data for the general Chinese population together with high frequency of CYP2C19 and CYP3A4 EM in Chinese (Barter et al., 2013). The hepatic CYP abundances (mean and CV), genotypes and corresponding phenotype frequencies obtained from several studies are summarized in Table 1. Given different phenotypes of CYP2B6 were integrated in the simulation, two separate Monte Carlo analyses were performed for EM and PM, respectively. 15000 simulations were run to predict the probability distribution of $\mathrm{C}_{\max }$ in the heart venous blood of R- and S-methadone for each CYP2B6 phenotype population. The distribution parameters for the whole population were calculated by using the weighted average parameters obtained in each phenotype population. Weighting was based on the EM and PM phenotype frequencies in the two population as shown in Table 1. In the model simulation the parameters were allowed to vary independently from each other. The summary of distribution parameters was shown in Table S8. Model codes of Monte Carlo simulation are provided in the supplementary materials 3 . Statistical analysis of the population distributions obtained with Monte Carlo simulations was performed in GraphPad Prism 5.0 (GraphPad Software Inc., San Diego, USA) calculating the GM, geometric CV, the $95^{\text {th }}$ and $99^{\text {th }}$ percentile of the $\mathrm{C}_{\max }$ in the heart venous blood of R- and S-methadone for the two populations.

\subsection{Derivation of CSAF}

Given that the IPCS (2005) guideline recommends several options for the calculation of CSAFs, different approaches were applied for deriving CSAFs to provide comprehensive information for the risk assessment of methadone. Firstly, the CSAF values for both the Caucasian and the Chinese population were derived by dividing the $95^{\text {th }}$ and the $99^{\text {th }}$ percentile of the $C_{\max }$ in the 
heart venous blood of R-and S-methadone by the GM of the $\mathrm{C}_{\max }$ of R-and S-methadone. Additionally, the Chinese population was considered as the sensitive group, and the CSAF values were calculated by dividing the $95^{\text {th }}$ and $99^{\text {th }}$ percentile of the $\mathrm{C}_{\max }$ in the heart venous blood of both enantiomers in the Chinese population by the GM of the $\mathrm{C}_{\max }$ of the respective enantiomers in the Caucasian population (IPCS, 2005). The CSAF values calculated based on the $99^{\text {th }}$ percentile in each population were used to generate in vivo dose-response curves of Rand S-methadone for sensitive groups ( $99^{\text {th }}$ percentile of $\mathrm{C}_{\max }$ ) in each population as outlined in the next section. In the present study definition of the sensitive groups is based on differences between individuals in methadone metabolism and does not cover any dynamic variation.

\subsection{PBK modelling-based reverse dosimetry and Benchmark dose analysis of in vivo cardiotoxicity predictions}

In our previous study (Shi et al., 2020a), PBK model-based reverse dosimetry was applied to translate in vitro concentration-response curves for rac-methadone-induced cardiotoxicity to in vivo dose-response curves for QTc prolongation. A similar approach was applied to predict the in vivo cardiotoxicity of methadone enantiomers for the average and sensitive groups in Caucasian and Chinese populations. To this end, the in vitro cardiotoxic effects of racmethadone on the FPDc measured in hiPSC-CMs using the MEA technique (Shi et al., 2020a) were used to derive concentration-response curves for R- and S-methadone. Given that Smethadone blocked hERG currents 3.5-fold more potently than R-methadone (Eap et al., 2007), the responses induced by rac-methadone were with the ratio of $1: 3.5$ proportionally distributed to responses of R- and S- methadone, respectively. Subsequently, the $f_{u}$ value of 0.79 for racmethadone in the in vitro medium of hiPSC-CM MEA assay (Shi et al., 2020a), assumed to be the same for R- and S-methadone, was used to calculate unbound in vitro R- and S-methadone concentrations which were set equal to the unbound steady-state $\mathrm{C}_{\max }$ of $\mathrm{R}$ - and S-methadone in the heart venous blood of the PBK model using the average of individual HLMs as specified by Shi et al. (2020a). Reverse dosimetry on each concentration-effect level tested in the hiPSCCMs was performed using the PBK models for the average Caucasian and the average Chinese population using the average $\mathrm{V}_{\max }$ and $\mathrm{K}_{\mathrm{m}}$ values obtained from incubations with the 25 Caucasian and 25 Chinese human liver microsomes, generating in vivo dose-response data for R- and S-methadone. From this the dose-response curves for R- and S-methadone were defined for the average Caucasian and the average Chinese population. The dose-response curves for sensitive groups in the Caucasian and the Chinese population were obtained by applying the 
respective CSAFs (calculated using the $99^{\text {th }}$ percentile of $\mathrm{C}_{\max }$ ) to the dose-response curves of the average populations given that no saturation occurs at the higher doses.

Benchmark dose (BMD) analysis of the predicted dose-response curves for R- and Smethadone was performed to obtain a benchmark dose resulting in $10 \%$ cardiotoxic effect $\left(\mathrm{BMD}_{10}\right)$ for the general and sensitive groups in the Caucasian and Chinese population, where the FPDc derived in vitro, can be regarded a representative endpoint for the QTc interval in the human ECG. An effect size of 10\% was chosen considering the physiological and statistical meaning of the abnormal QTc prolongation as previously described (Shi et al., 2020a). The BMD analysis was performed using the European Food Safety Authority web-tool integrated with the R-package PROAST version 66.90 developed by the Dutch National Institute for Public Health and the Environment (RIVM) as previously described (Shi et al., 2020b). Unlike the concentration-response curve itself, the accompanying confidence intervals of racmethadone could not be assigned or distributed to the two enantiomers. Therefore the BMDL 10 values (lower 95\% confidence limit of $\mathrm{BMD}_{10}$ ) were derived by dividing BMD values by 3 given that a reliable BMDL value should be at most 3-fold lower than the corresponding BMD value (EPA, 2012). BMDL 10 values were defined for the average group and for the sensitive group using the CSAF, which allows the extrapolation of inter-individual kinetic variations in metabolic conversion to variation in external toxic dose levels.

The Margin of safety (MOS) is an important concept in the safety evaluation of drugs. In the classic approach, the MOS for the drug safety in pharmaceutical industry is the ratio of the lethal dose or toxic dose to $1 \%$ of the population $\left(\mathrm{LD}_{1}\right.$ or $\left.\mathrm{TD}_{1}\right)$ to the effective dose to $99 \%$ of the population $\left(\mathrm{ED}_{99}\right)$ and would require in vivo data representative for the population. To obtain insight in the influence of inter-individual variation on the toxicological profile for the risk-benefits of a compound earlier in the drug developmental process BMDLs derived using the presented in vitro-in silico approach can be integrated in the MOS approach. In this example the predicted $\mathrm{BMDL}_{10}$ of the enantiomers for the sensitive population $\left(99^{\text {th }}\right.$ percentile of the $\mathrm{C}_{\max }$ in the heart venous blood) was chosen as an alternative to the $\mathrm{TD}_{1}$ and the therapeutic dose of rac-methadone served as $\mathrm{ED}_{99}$ because information on therapeutic doses of enantiomers is not available. 


\section{Results}

\subsection{Metabolic variation in the conversion of $\mathbf{R}$ - and S-methadone}

\subsubsection{In vitro incubation of 25 Caucasian and 25 Chinese individual liver microsomes}

The conversion of R- and S- methadone toward R- and S-EDDP was measured in incubations with individual microsomes originating from the Caucasian and the Chinese population. The concentration-dependent increase in the formation of R- and S-EDDP followed MichaelisMenten kinetics. The obtained apparent $\mathrm{V}_{\max }$ and $\mathrm{K}_{\mathrm{m}}$ values, and the calculated catalytic efficiencies derived from the data are summarized in Table 2 and individual results are shown in Table S9. For the 25 Caucasian individuals, the differences between the individuals with the highest and lowest catalytic efficiency and the $\mathrm{CV}$ of the inter-individual differences in catalytic efficiency for R-methadone were 1.9- and 1.4-fold lower than the ones for Smethadone, respectively. The mean catalytic efficiency for R-methadone conversion to REDDP was 1.5-fold lower than that for S-methadone conversion. For the 25 Chinese individuals, a comparable variation in the metabolism of R- and S-methadone was observed for differences between the highest and lowest catalytic efficiency, the CV of the interindividual differences in catalytic efficiency and the mean catalytic efficiency.

Regarding the inter-ethnic variations in the metabolism of R-methadone, a 6.3-fold higher mean $\mathrm{V}_{\max }$ value and a comparable mean $\mathrm{K}_{\mathrm{m}}$ value were obtained for the Caucasian population compared to the Chinese population, resulting in a 5.2-fold higher mean catalytic efficiency in the Caucasian population than the Chinese population (Table 2). For the metabolism of Smethadone, the catalytic efficiency was 9-fold higher in the Caucasian population than in the Chinese population, which is mainly due to a 7.4-fold higher mean $\mathrm{V}_{\max }$ value since similar mean $\mathrm{K}_{\mathrm{m}}$ values were observed in the Caucasian population compared to the Chinese population (Table 2). The CV of the catalytic efficiency for R- and S-methadone metabolism was respectively 1.4- and 2.2-fold higher in the Caucasian population than in the Chinese population. 
Table 2 Descriptive statistic of the kinetic constants $V_{\max }, K_{m}$ and catalytic efficiencies for $R$ EDDP and S-EDDP formation by 25 Caucasian and 25 Chinese individual human liver microsomes.

\begin{tabular}{|c|c|c|c|c|c|c|c|c|c|c|c|c|}
\hline & \multicolumn{6}{|c|}{ Caucasian individuals } & \multicolumn{6}{|c|}{ Chinese individuals } \\
\hline & \multicolumn{3}{|c|}{ R-EDDP formation } & \multicolumn{3}{|c|}{ S-EDDP formation } & \multicolumn{3}{|c|}{ R-EDDP formation } & \multicolumn{3}{|c|}{ S-EDDP formation } \\
\hline & $\begin{array}{l}\mathrm{V}_{\mathrm{ma}} \\
\mathrm{x}^{\mathrm{a}}\end{array}$ & $\mathrm{K}_{\mathrm{m}}^{\mathrm{b}}$ & $\begin{array}{l}\text { Catalyti } \\
\text { c } \\
\text { efficien } \\
\text { cy }^{c}\end{array}$ & $\begin{array}{l}\mathrm{V}_{\mathrm{ma}} \\
\mathrm{x}^{\mathrm{a}}\end{array}$ & $\mathrm{K}_{\mathrm{m}}^{\mathrm{b}}$ & $\begin{array}{l}\text { Catalyti } \\
\mathrm{c} \\
\text { efficien } \\
\mathrm{cy}^{\mathrm{c}}\end{array}$ & $\begin{array}{l}\mathrm{V}_{\mathrm{ma}} \\
\mathrm{x}^{\mathrm{a}}\end{array}$ & $\mathrm{K}_{\mathrm{m}}{ }^{\mathrm{b}}$ & $\begin{array}{l}\text { Catalyti } \\
\text { c } \\
\text { efficien } \\
\text { cy }^{c}\end{array}$ & $\begin{array}{l}\mathrm{V}_{\mathrm{ma}} \\
\mathrm{x}^{\mathrm{a}}\end{array}$ & $\mathrm{K}_{\mathrm{m}}{ }^{\mathrm{b}}$ & $\begin{array}{l}\text { Catalyti } \\
\text { c } \\
\text { efficien } \\
\text { cy }^{c}\end{array}$ \\
\hline $\begin{array}{l}\text { Mean } \\
\left(\mu_{x}\right)\end{array}$ & 0.40 & $\begin{array}{l}155 . \\
1\end{array}$ & 2.87 & 0.34 & $\begin{array}{l}111 . \\
3\end{array}$ & 4.27 & $\begin{array}{l}0.06 \\
4\end{array}$ & $\begin{array}{l}127 . \\
7\end{array}$ & 0.55 & $\begin{array}{l}0.04 \\
6\end{array}$ & $\begin{array}{l}115 . \\
6\end{array}$ & 0.47 \\
\hline $\mathrm{SD}^{\mathrm{d}}$ & $\begin{array}{l}0.32 \\
4\end{array}$ & 42.8 & 2.64 & $\begin{array}{l}0.31 \\
9\end{array}$ & 44.2 & 5.54 & $\begin{array}{l}0.03 \\
1\end{array}$ & 42.4 & 0.35 & $\begin{array}{l}0.02 \\
1\end{array}$ & 63.6 & 0.28 \\
\hline $\mathrm{CV}_{\mathrm{x}} \%^{\mathrm{e}}$ & 80.3 & 27.6 & 92.1 & 93.8 & 40 & 130 & 48.5 & 33.2 & 64.3 & 46.5 & 55 & 59.8 \\
\hline $\begin{array}{l}\text { Fold- } \\
\text { differen } \\
\operatorname{ces}^{f}\end{array}$ & 13 & 3 & 19 & 17 & 4 & 37 & 11 & 4 & 10 & 11 & 8 & 12 \\
\hline
\end{tabular}

${ }^{\mathrm{a}} \mathrm{nmol} / \mathrm{min} / \mathrm{mg}$ liver microsomes, ${ }^{\mathrm{b}} \mu \mathrm{M},{ }^{\mathrm{c}} \mathrm{V}_{\max } / \mathrm{K}_{\mathrm{m}}, \mu \mathrm{l} / \mathrm{min} / \mathrm{mg}$ protein, ${ }^{\mathrm{d}}$ standard deviation of kinetic constants, ${ }^{\mathrm{e}}$ coefficient of variation, $\%$ (= SD/mean $\left.\times 100\right),{ }^{\mathrm{f}}$ highest/lowest values.

\subsubsection{Kinetic constants for $R$ - and $S$-methadone conversion by rCYPs and variations in CYP abundances in the Caucasian and the Chinese population}

Table 1 shows the in vitro kinetic constants $\mathrm{V}_{\max }$ and $\mathrm{K}_{\mathrm{m}}$ for the conversion of methadone enantiomers to EDDP enantiomers by the major CYPs as reported by Totah et al. (2007). The Table also presents the scaled catalytic efficiency for Caucasian and Chinese HLM for R- and S-methadone conversion taking into account the ISEF and the population specific CYP abundances to calculate $\mathrm{V}_{\max }$ according to equation 2. Compared to the Chinese population, the Caucasian population has 3.2-fold higher, 2.5-fold higher and 1.3-fold lower abundances in CYP2B6, CYP2C19 and CYP3A4, respectively, with larger CVs.

\subsection{Sensitivity analysis and evaluation of the PBK model for R- and S- methadone}

The sensitivity analysis shows that the SC of model parameters in the PBK model using the average individual HLM kinetic data were similar for R-and S-methadone at the two dose levels analysed (supplementary materials 1, Figure S1). For both the Caucasian and the Chinese PBK model, the predicted steady-state $\mathrm{C}_{\max }$ of R- or S-methadone in the heart venous blood was highly influenced by the following model parameters with the hierarchy of normalized SC 
values being the oral fraction absorbed $(\mathrm{Fa})>$ body weight $(\mathrm{BW})>$ liver metabolism related parameters (VLc, MPPGL, $\mathrm{V}_{\max }, \mathrm{K}_{\mathrm{m}}$ ) $>$ the absorption rate constant (ka). The renal clearance (RCL) was more influential in the Chinese model with a normalized SC value comparable to those of liver metabolism related parameters. The results of the sensitivity analysis performed in the PBK model using rCYPs kinetic data were similar to the results obtained in the PBK model using HLM kinetic data (data not shown).

The developed PBK models using the average individual HLM kinetic data or rCYPs kinetic data for R- and S-methadone were evaluated against reported in vivo human data. Figure 2 reveals that for both models the predicted blood concentrations of R- and S-methadone during the last $24 \mathrm{~h}$ upon a repeated oral rac-methadone dose of $100 \mathrm{mg}$ /day for 30 days, adequately matched with the corresponding in vivo data for Caucasian subjects (Liu et al., 2007). A similar comparison was obtained between predictions and in vivo human data from other studies (Foster et al., 2000; Garimella et al., 2015) as shown in Figure S2 and S3 in the supplementary materials 1. Compared to data from three in vivo studies, when using the HLM kinetic data and rCYPs kinetic data, the prediction of kinetic values of R-methadone showed a 0.79 - to 1.06fold difference in steady-state $\mathrm{C}_{\max }$ in venous blood and a 0.67-to 0.91-fold difference in AUC values. In case of S-methadone, the prediction showed a 0.95 - to 1.36 - fold difference in steadystate $\mathrm{C}_{\max }$ in venous blood and a 0.75-to 1.08-fold difference in AUC values (Table S6 and S7 in the supplementary materials 1 ). 

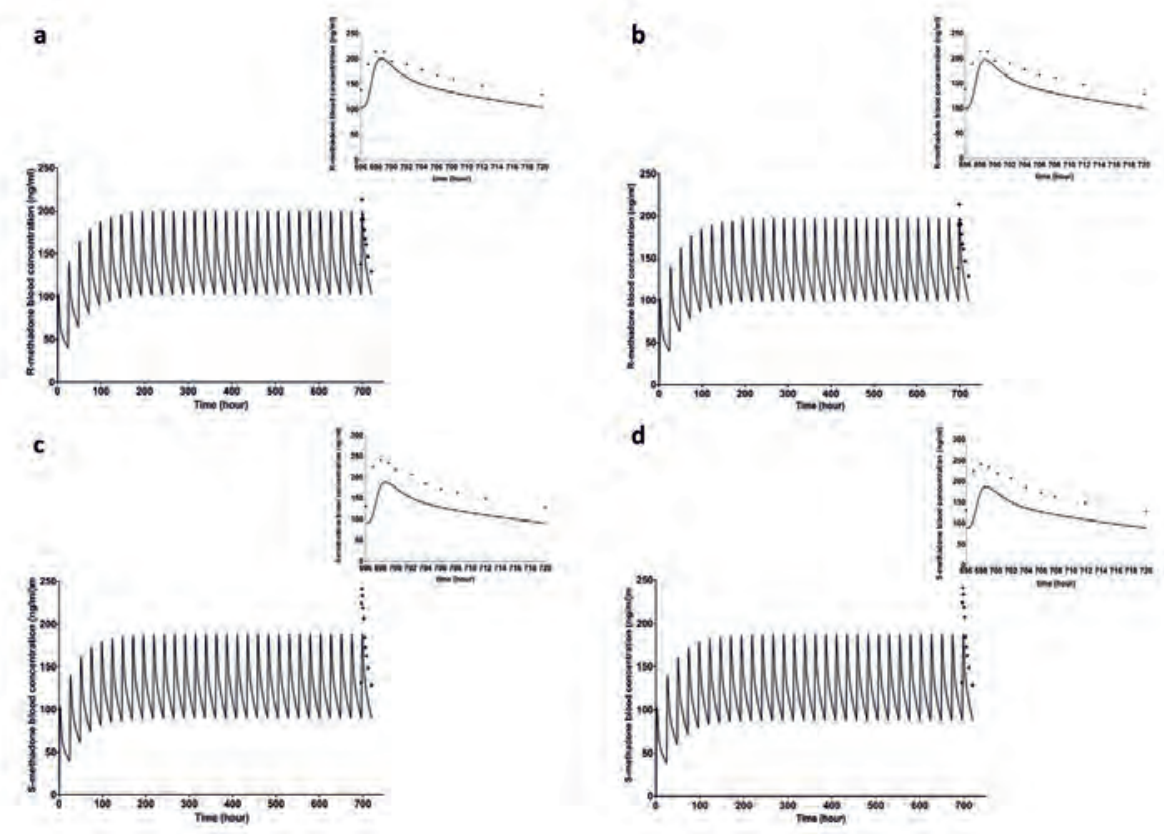

Figure 2 Blood concentration-time curves of R-methadone (a, b) and S-methadone (c, d) in human predicted with the PBK model (lines) and published in vivo data (dots) (Liu et al., 2007) after a repeated oral rac-methadone dose of $100 \mathrm{mg} /$ day for 30 days. (a) and (c) present predictions obtained from the model using HLM kinetic data and (b) and (d) present predictions obtained from the model using rCYPs kinetic data. The top right insert is the predicted blood concentration of R- and Smethadone (lines) and in vivo data (dots) during the last $24 \mathrm{~h}$ upon the oral exposure.

\subsection{Prediction of inter-individual and inter-ethnic variations applying individual PBK models and PBK modelling combined with Monte Carlo simulations}

Figure 3 shows the differences in the distribution of the predicted $\mathrm{C}_{\max }$ of $\mathrm{R}$ - and S-methadone in the heart venous blood among 25 Caucasian and 25 Chinese individuals. In the 25 Caucasian individuals the geometric $\mathrm{CV}$ of the predicted $\mathrm{C}_{\max }$ of $\mathrm{R}$-methadone in the heart venous blood was 1.6-fold lower than that of S-methadone, while in the 25 Chinese individuals, this value was 1.2-fold lower for R-methadone compared to S-methadone. For the inter-ethnic variations, the GM of predicted $\mathrm{C}_{\max }$ of R- and S-methadone in 25 Caucasian individuals was 2.2- and 3fold lower than those in 25 Chinese individuals, respectively, and the geometric CVs observed 
in Caucasian individuals were 2.1 and 2.8-fold higher than those observed in Chinese individuals for R- and S-methadone, respectively.

The inter-individual and inter-ethnic differences in $\mathrm{C}_{\max }$ of $\mathrm{R}$ - and S-methadone in the heart venous blood predicted with the Monte Carlo simulations using variation in CYP abundances are shown in Figure 3. Both for the Caucasian and the Chinese population, the geometric CVs and the differences between highest and lowest predicted $\mathrm{C}_{\max }$ of $\mathrm{R}$ - and S-methadone were comparable to those obtained from individual PBK models, except that the geometric CVs of predicted $\mathrm{C}_{\max }$ of $\mathrm{S}$-methadone in the Caucasian population was 1.8-fold lower than the results obtained from the individual Caucasian PBK models. For the Caucasian population, the GM of $\mathrm{C}_{\max }$ of the two enantiomers were comparable with the GM values predicted using the individual PBK models and for the Chinese population the GM of predicted $\mathrm{C}_{\max }$ of R- and Smethadone were both 1.1-fold higher than when using individual PBK models. Furthermore, the inter-ethnic variations in GM and geometric CVs of predicted $\mathrm{C}_{\max }$ of the two enantiomers differ less than 2-fold from the ones obtained using individual PBK models. Detailed predictions are shown in Table S10 in the supplementary materials 1.
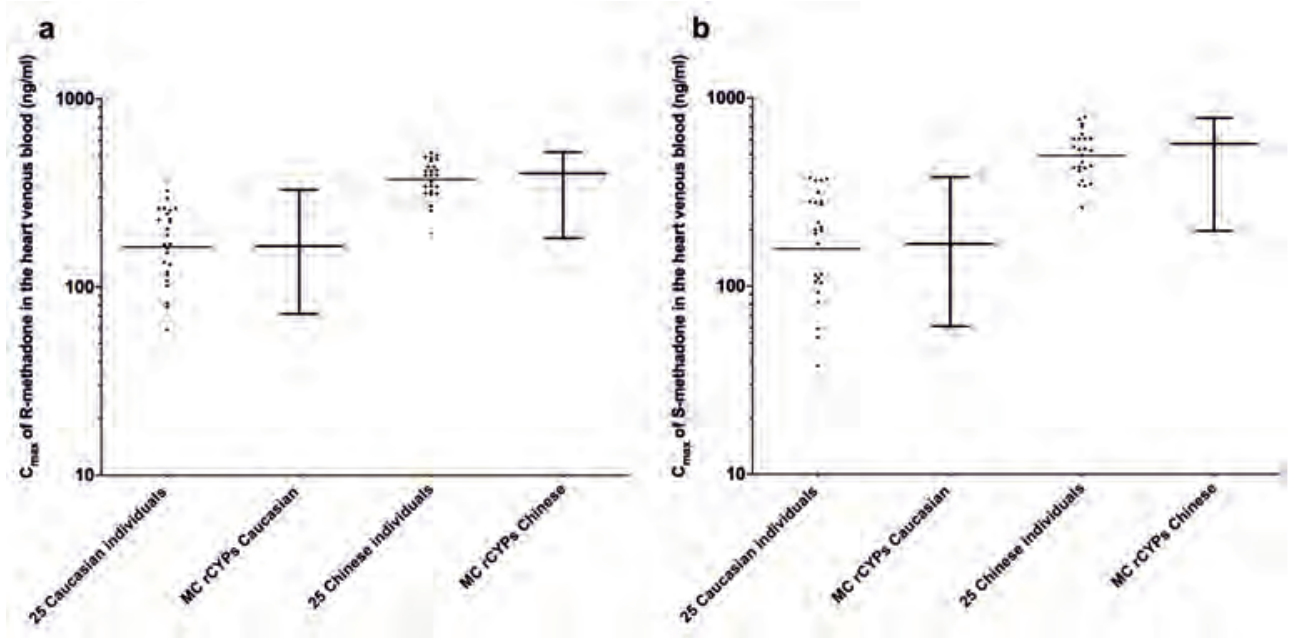

Figure 3 Distribution of predicted $C_{\max }$ of $R$ - (a) and S-methadone (b) in the heart venous blood at steady-state after a repeated oral methadone enantiomer dose of $30 \mathrm{mg} / \mathrm{day}$ for 30 days in the Caucasian and the Chinese population. The scatter plots represent the predictions obtained using individual PBK models. Box and whisker plots represent the predictions obtained by the Monte Carlo (MC) simulation using reported in vitro kinetic data of rCYPs. The whiskers represent the $1^{\text {st }}$ and $99^{\text {th }}$ percentile of defined populations. 


\subsection{Derivation of CSAF}

The frequency distribution of the $\mathrm{C}_{\max }$ of methadone enantiomers in the heart venous blood is shown in Figure 4. Table 3 shows the CSAF values calculated for the Caucasian population, the Chinese population and the two populations combined. The CSAFs for the Chinese were 1.4 to 1.6 -fold lower than the Caucasian CSAFs, indicating a smaller inter-individual variation in the Chinese compared to the Caucasian population, which is also visible in Figure 3.
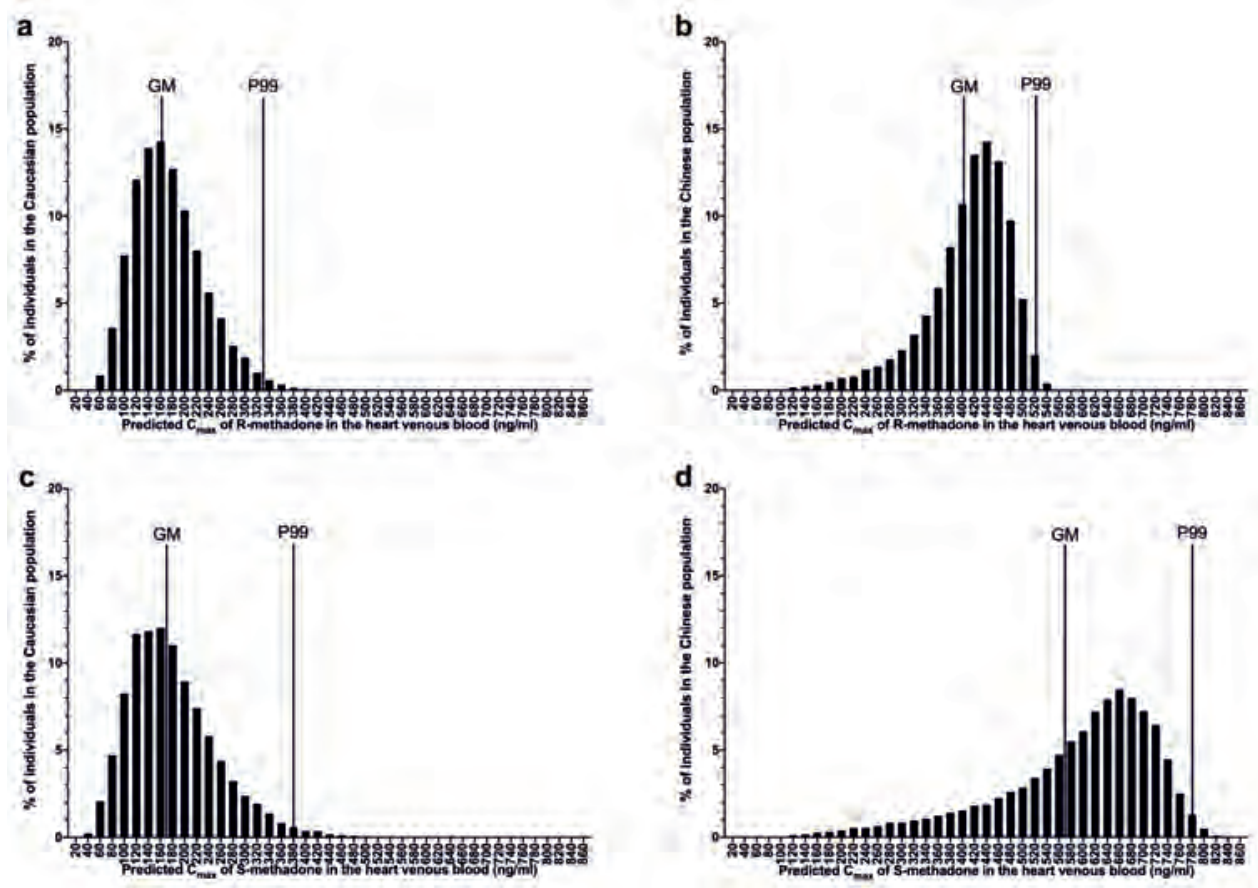

Figure 4 Frequency distribution for $C_{\max }$ of $R$ - and $S$-methadone in the heart venous blood at steady-state after a repeated oral methadone enantiomer dose of $30 \mathrm{mg} / \mathrm{day}$ for 30 days in Caucasian (a, c) and Chinese (b, d) individuals by the Monte Carlo simulation using in vitro kinetic data of rCYPs. The GM and P99 represent the geometric mean and the $99^{\text {th }}$ percentile of the distribution. 
Table 3 CSAFs of R- and S-methadone for the Caucasian population, the Chinese population and the two populations combined in each scenario of Monte Carlo simulation.

\begin{tabular}{|c|c|c|c|c|c|c|}
\hline & \multicolumn{3}{|c|}{ CSAFs at $95^{\text {th }}$ percentile } & \multicolumn{3}{|c|}{ CSAFs at $99^{\text {th }}$ percentile } \\
\hline & $\begin{array}{c}\text { Caucasian } \\
\text { population } \\
\text { a }\end{array}$ & $\begin{array}{c}\text { Chinese } \\
\text { population }^{\mathrm{a}}\end{array}$ & $\begin{array}{c}\text { Two } \\
\text { populations } \\
\text { combined }^{\mathrm{b}}\end{array}$ & $\begin{array}{l}\text { Caucasian } \\
\text { population }^{\text {a }}\end{array}$ & $\begin{array}{c}\text { Chinese } \\
\text { population }^{\mathrm{a}}\end{array}$ & $\begin{array}{c}\text { Two } \\
\text { populations } \\
\text { combined }^{\mathrm{b}}\end{array}$ \\
\hline $\begin{array}{c}\mathrm{R}- \\
\text { methadone }\end{array}$ & 1.7 & 1.2 & 3.0 & 2.0 & 1.3 & 3.2 \\
\hline $\begin{array}{c}\text { S- } \\
\text { methadone }\end{array}$ & 1.9 & 1.3 & 4.5 & 2.3 & 1.4 & 4.7 \\
\hline
\end{tabular}

a obtained by dividing the $95^{\text {th }}$ or $99^{\text {th }}$ percentile of the $C_{\max }$ in heart venous blood by the GM of the $\mathrm{C}_{\max }$ in heart venous blood in each population. ${ }^{\mathrm{b}}$ obtained by dividing the $95^{\text {th }}$ or $99^{\text {th }}$ percentile of the $\mathrm{C}_{\max }$ in heart venous blood in the Chinese population by the GM of the $\mathrm{C}_{\max }$ in heart venous blood in the Caucasian population.

\subsection{PBK modelling-based reverse dosimetry and BMD analysis of in vivo cardiotoxicity of methadone}

PBK modelling-based reverse dosimetry was applied to further investigate the consequences of obtained inter-individual and inter-ethnic kinetic variations for the predicted in vivo cardiotoxicity of R- and S-methadone. To this end, first the in vitro derived cardiotoxicity of rac-methadone obtained in the hiPSC-CM MEA assay (Shi et al., 2020a) was transformed to cardiotoxicity data for the individual R- and S-enantiomer, based on the reported hERG channel inhibition potencies of the two enantiomers (Eap et al., 2007). The thus obtained in vitro concentration-response curves of R- and S-methadone are shown in Figure S4.

After reverse dosimetry, the in vivo dose-response curves for R- and S-methadone for the average and sensitive population for the both the Caucasian and Chinese population (Figure 5) indicate a larger variation in both R- and S-methadone-induced human cardiotoxicity for the Caucasian population compared to in the Chinese population. Table 4 shows that the BMDL 10 value of R- and S-methadone for the average Caucasian were respective 2.1- and 2.4-fold higher than the $\mathrm{BMDL}_{10}$ values of the sensitive Caucasians. For the average Chinese the $\mathrm{BMDL}_{10}$ value of two enantiomers were 1.4-fold higher than the $\mathrm{BMDL}_{10}$ values for the of sensitive Chinese. BMDL 10 values of S-methadone for the average and the sensitive Caucasians were respectively 3.7- and 2-fold higher than the corresponding values for the Chinese, indicating that the Caucasians may be less sensitive to methadone-induced cardiotoxicity of than the Chinese (Table 4). The predicted MOS values are summarized in Table 5. For both enantiomers the MOS values for the Caucasians were 1.6- to 2-fold higher 
than the MOS values for the Chinese. The MOS values for R-methadone were generally 7-fold higher than the ones for S-methadone.

Table 4 The predicted $\mathrm{BMDL}_{10}$ values for the average and the sensitive $\left(99^{\text {th }}\right.$ percentile of predicted $\mathrm{C}_{\max }$ in heart venous blood) of the Caucasian and Chinese population obtained by the CSAFs derived from the Monte Carlo simulation.

\begin{tabular}{llllll}
\hline & \multicolumn{2}{c}{ Caucasian population } & & \multicolumn{2}{l}{ Chinese population } \\
\cline { 1 - 2 } \cline { 5 - 6 } & R-methadone & $\begin{array}{l}\text { S- } \\
\text { methadone }\end{array}$ & & R-methadone & S-methadone \\
\hline $\begin{array}{l}\text { BMDL10 (mg/day) for } \\
\text { the average population }\end{array}$ & 99.6 & 18.7 & 39.5 & 5.1 \\
$\begin{array}{l}\text { BMDL } 10 \text { (mg/day) for } \\
\text { sensitive population }\end{array}$ & 47.4 & 7.5 & 29.9 & 3.7 \\
\hline
\end{tabular}

Table 5 Summary of Margin of Safety values for $R$ and S-methadone for the Caucasian and Chinese population. The Margin of Safety is defined as the ratio the predicted BMDL 10 of the enantiomers for the sensitive population $\left(99^{\text {th }}\right.$ percentile of the $C_{\max }$ in the heart venous blood) and the therapeutic dose of rac-methadone.

\begin{tabular}{|c|c|c|c|c|c|c|}
\hline \multirow[t]{2}{*}{ Enantiomer } & \multirow[t]{2}{*}{ Stage dosing } & \multirow{2}{*}{$\begin{array}{l}\text { Effective dose } \\
\text { (rac- } \\
\text { methadone } \\
\text { mg/day) }\end{array}$} & Tox & mg/day) & \multicolumn{2}{|c|}{ Margin of Safety ${ }^{b}$} \\
\hline & & & Caucasian & Chinese & Caucasian & Chinese \\
\hline \multirow{2}{*}{$\begin{array}{l}\text { R- } \\
\text { methadone }\end{array}$} & Initial & 10 & 47.4 & 29.9 & 4.7 & 3.0 \\
\hline & Maintenance & 60 & 47.4 & 29.9 & 0.8 & 0.5 \\
\hline \multirow{2}{*}{$\begin{array}{c}\text { S- } \\
\text { methadone }\end{array}$} & Initial & 10 & 7.5 & 3.7 & 0.7 & 0.4 \\
\hline & Maintenance & 60 & 7.5 & 3.7 & 0.12 & 0.06 \\
\hline
\end{tabular}

${ }^{\mathrm{a}} \mathrm{BMDL}_{10}$ values for the sensitive population $\left(99^{\text {th }}\right.$ percentile of $\mathrm{C}_{\max }$ in heart venous blood) were used as toxic dose for $1 \%$ population $\left(\mathrm{TD}_{1}\right) .{ }^{\mathrm{b}}$ obtained by dividing $\mathrm{TD}_{1}$ by effective dose. 

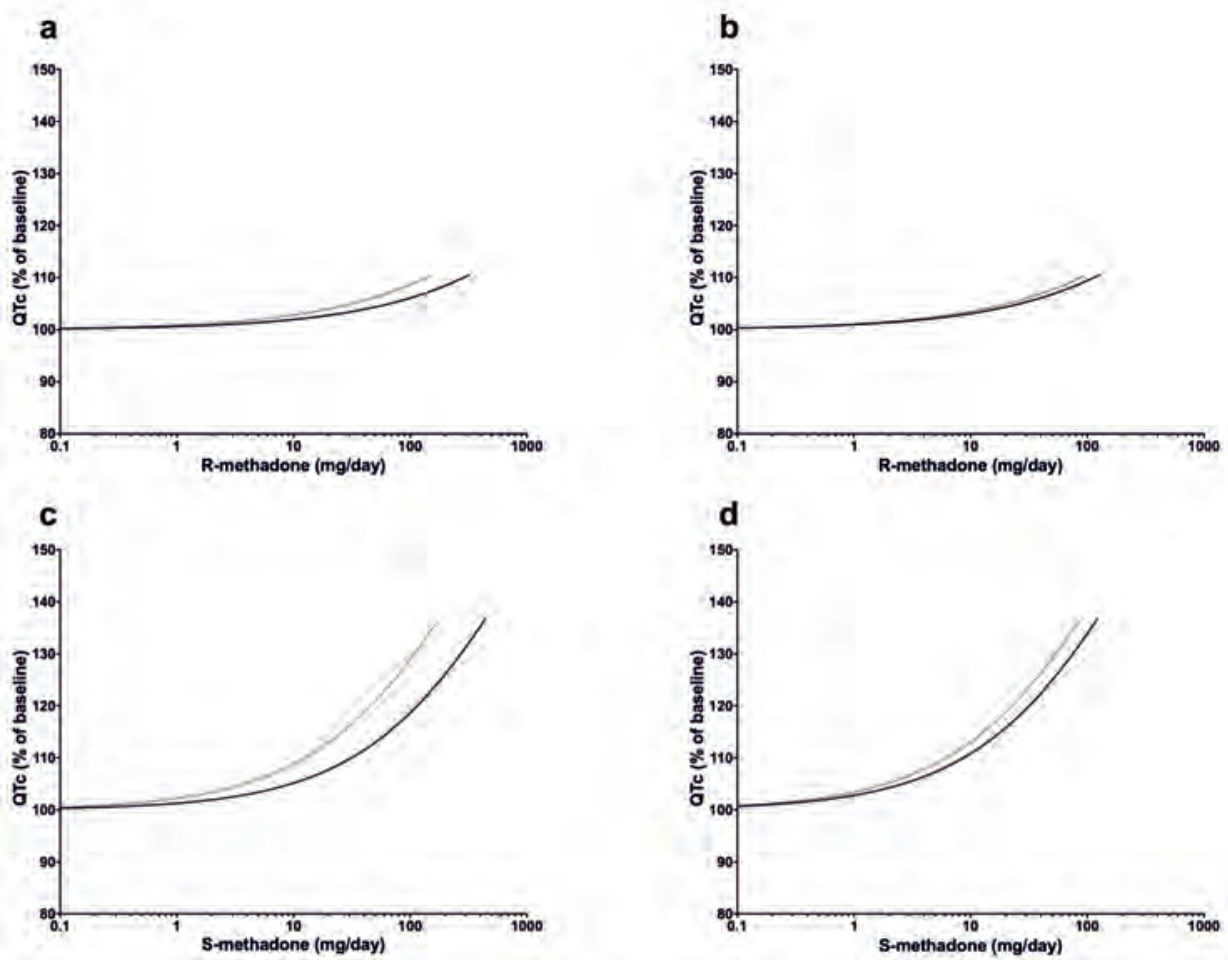

Figure 5 Predicted dose-response curves for the cardiotoxicity of R-methadone and S-methadone in the average (solid lines) and the sensitive population $\left(99^{\text {th }}\right.$ percentile of predicted $C_{\max }$ in heart venous blood) (dotted lines) of Caucasian (a) (c) and Chinese (b) (d) population. The dose-response curves for sensitive Caucasian and Chinese populations were obtained by applying the respective CSAFs to the dose-response curves of the average populations.

\section{Discussion}

The aim of present study was to apply a NAM approach that combines in vitro data, PBK modelling and Monte Carlo simulations to predict inter-ethnic and inter-individual kinetic variations in the R- and S-methadone-induced cardiotoxicity, and to elucidate consequences of these variations for the sensitivity towards the cardiotoxicity of both enantiomers. CSAFs were derived to quantitatively reflect the inter-ethnic and inter-individual variation in kinetics and used to derive dose-response curves for the sensitive individual in the population. Applying the presented NAM in drug safety evaluation contributes to the $3 \mathrm{Rs}$, since the approach reduces the need for animal studies on cardiotoxicity.

In the current paper, two sources of metabolic data were integrated in the PBK model to define the inter-individual differences in the metabolism of R- and S-methadone. Firstly, the 
formation of both enantiomers was determined with 25 Caucasian and 25 Chinese individual HLMs and kinetic constants obtained were used to define 50 individual PBK models. The catalytic efficiencies for the metabolism of both enantiomers were 5- to 9-fold higher in incubations with the Caucasian HLMs compared to Chinese HLMs, and, as a result, the PBK model predictions revealed a notable inter-ethnic difference in the predicted venous blood concentrations. The observed differences may be explained by the inter-ethnic differences in the distribution of functional alleles and in the abundance of CYP2B6 and CYP3A. Zhou et al. (2017) found that the overall frequency of CYP2B6 alleles, associated with higher catalytic activity, was 2-fold higher in Europeans compared to the East Asians. Especially, two key allelic mutations $(516 \mathrm{G}>\mathrm{T}$ and $785 \mathrm{~A}>\mathrm{G})$, known to increase the in vitro catalytic efficiency for conversion of 7-ethoxy-4-trifluoromethylcoumarin (Jinno et al., 2003) and cyclophosphamide (Xie et al., 2003), have higher frequencies in the Caucasian population compared to the Chinese population (Guan et al.,2006). The CYP3A4*20 allele, reported to result in inactived catalytic activity (Zhou et al., 2017), showed a 3.7-fold lower prevalence in the Caucasian population compared to the East Asians (McGraw and Waller, 2012) and the CYP3A4*22 allele, which decreases the activity and protein expression, had a higher frequency in the Asian population (0.043) compared to the Caucasians (0.008-0.025) (Zanger and Schwab, 2013). Furthermore, the reported hepatic abundances of CYP2B6 and CYP3A4 (EM) were up to 3-fold higher in Caucasians compared to the Chinese (Barter et al., 2013).

Another factor contributing to the ethnic differences in the catalytic efficiency and predicted kinetics could be the content of cytochrome b5 (Cyt b5). It is important to note that the Cyt b5 plays an important role in the CYP-mediated reactions where Cyt b5 may provide the second electron to the monooxygenase cycle (Kandel and Lampe, 2014). Many studies indicated that Cyt b5 stimulated the catalytic activity of CYP2B6 and CYP3A4. Yamazaki et al. (1996) demonstrated that the catalytic efficiency for testosterone (CYP3A4 substrate) in the presence of Cyt b5 was 4-fold higher than the one without Cyt b5. Zhang et al. (2015a) found a positive correlation between the Cyt b5 content and the catalytic efficiency (and $\mathrm{V}_{\max }$ ) for CYP2B6 in Chinese HLM, which was in agreement with the positive correlation we found between the Cyt b5 content and the catalytic efficiency (and $\mathrm{V}_{\max }$ ) for both enantiomers in 25 Caucasian and 25 Chinese individual HLMs (Figure S5), suggesting that Cyt b5 may influence the catalytic efficiency for methadone enantiomers. The mean Cyt b5 content in HLMs used in the current study are $455 \mathrm{pmol} / \mathrm{mg}$ for Caucasians HLMs and $199 \mathrm{pmol} / \mathrm{mg}$ for Chinese, which are comparable with reported values being $660 \mathrm{pmol} / \mathrm{mg}$ for Caucasians (Corning, 2014) and 270 
$\mathrm{pmol} / \mathrm{mg}$ for Chinese (Zhang et al., 2015a). Thus, the lower conversion of methadone by the Chinese HLM may in part also be ascribed to the lower Cyt b5 content.

Our results reveal that Caucasians had in general 2-fold higher CVs for the catalytic efficiency and predicted venous blood concentrations for both enantiomers than Chinese. This can be partly explained by the fact that the CV of CYP3A4 abundance is 3-fold higher for Caucasians compared to Chinese (Achour et al., 2014, Shu et al., 2000), and that the allelic variants of CYP2B6 and CYP3A4 appeared to be more frequent in Caucasian than in Asian (Li and Bluth, 2011). The latter fact may also explain the larger variations in the kinetics of Smethadone within the Caucasian population observed in the current study, given that CYP2B6 shows stereoselectivity towards to metabolism of S-methadone (Chang et al., 2011).

The reported CYP abundances combined with kinetic data for the respective rCYPs were used as the second source to describe inter-individual variation in metabolism of methadone enantiomers using a PBK model integrated with Monte Carlo simulations. Generally, the predicted $\mathrm{GM}$ of $\mathrm{C}_{\max }$ in the heart venous blood and the corresponding $\mathrm{CV}$ values were comparable with the results obtained from the individual PBK models, especially for the Caucasian population. For the Chinese population the predicted $\mathrm{GM}$ of $\mathrm{C}_{\max }$ in the heart venous blood for the two enantiomers showed a 1.2-fold differences between the two approaches. The reasons underlying this observation may be related to the fact that the ISEF values used for the Chinese models were derived using $\mathrm{f}_{\mathrm{m}, \mathrm{CYP}}$ obtained from Caucasian microsomes due to the absence of Chinese microsomal data. The derivation of ISEFs was reported to vary among studies and be dependent on the accessory proteins (Chen et al., 2011; Crewe et al., 2011). Crewe et al. (2011) demonstrated that ISEFs for CYP2C9 differed up to 10-fold between rCYP systems with and without Cyt b5, indicating that ISEFs were sensitive to the differences in Cyt b5 and the CYP450/Cyt b5 ratio can be used to indicate the influences of Cyt b5 variations on ISEFs. In the current study, the CYP450/Cyt b5 ratio of 25 Caucasian HLM is 1 while the ratio for 25 Chinese HLM was much higher amounting to around 10 (Table S1,). Given that both the Cyt b5 content and CYP450/Cyt b5 ratio differed between the two populations, the ISEFs determined based on Caucasian microsomal kinetics may not completely capture the discrepancy between rCYP system and HLM system for the Chinese population. Further studies on Chinese specific ISEFs are needed to improve the prediction. Moreover, together with reporting rCYPs catalytic data, assay specific ISEF values should ideally also be derived and reported, since the absence of these ISEFs hamper the use of reported rCYPs data, for example for QIVIVE. 
Altogether, comparing the results obtained from the individual PBK models and the Monte Carlo prediction indicates that both approaches similarly predict the inter-individual and interethnic variations in the kinetics of R-methadone and to a lesser extent S-methadone. This implies that both groups of 25 Caucasian and 25 Chinese individuals were able to represent the inter-ethnic kinetic variations between Caucasians and Chinese on a population level.

Comparing the CSAF values of the Caucasian and the Chinese to the default safety factor of 3.16 for kinetic differences used in chemical risk assessments (IPSC, 2005) indicates a limited inter-individual kinetic variation in each population for both enantiomers. When considering the combined population, the obtained CSAFs for S-methadone were somewhat higher than 3.16, but for R-methadone this default uncertainty factor for interindividual differences in kinetics appears sufficiently protective. For both enantiomers, the MOSs were 2-fold higher for the Caucasians compared to the Chinese, indicating that, based on the kinetic differences observed, the Chinese population may be at extra risk towards methadone-induced cardiotoxicity. The $\mathrm{BMDL}_{10}$ of rac-methadone for the average Caucasian was $24 \mathrm{mg} /$ day (Shi et al., 2020a). After applying the CSAF of rac-methadone obtained using the same approach the MOS values obtained were 4- to 9-fold lower than the MOS values of R-methadone for the Caucasians, indicating administering only R-methadone might decrease the risk of methadoneinduced cardiotoxicity, which is in agreement with the study of Ansermot et al. (2010) where the replacement of rac-methadone by R-methadone was shown to reduce the prolonged QTc interval in opioid addiction patients, thus the use of R-methadone is highly recommended in the clinic.

In the current study the inter-ethnic and inter-individual variation in methadone-induced cardiotoxicity were assessed using the variability in metabolic kinetics, which could to some extent reflect the overall variability in the populations given the significant role of variation in methadone blood concentrations in the variation in the individual sensitivity in the clinical settings (Eap et al., 2002; Li et al., 2008). HiPSC-CMs derived from different donors have been demonstrated as a potential tool to study the inter-individual variability in the toxicodynamics of drug-induced cardiotoxic effects (Burnett et al., 2021). To what extent a chemical shows such inter-individual variation in toxicodynamics for cardiotoxicity may be chemical-specific and also dependent on the type of dynamic endpoint quantified (e.g. QT prolongation, beating rate, peak amplitude and cell viability) (Blanchette et al., 2020; Burnett et al., 2019; Grimm et al., 2018). Due to lack of such data on methadone, toxicodynamic variations could not be included in the present approach, but future data on potential inter-ethnic and inter-individual 
variation in toxicodynamics could be combined with the data on inter-ethnic and interindividual variation in toxicokinetics defined in the present study to further characterize the inter-individual variation in methadone-induced cardiotoxicity and define an overall CSAF that also includes a CSAF for interindividual differences in toxicodynamics $\left(\mathrm{HD}_{\mathrm{AF}}\right)$.

In conclusion, we demonstrated that integrating in vitro cardiotoxicity data, PBK modelling and Monte Carlo simulation can be a powerful approach to predict the influence of inter-ethnic and inter-individual kinetic variations for the sensitivity towards on R- and S-methadoneinduced cardiotoxicity. PBK models based on either HLM kinetics or rCYPs kinetics similarly predicted the inter-ethnic and inter-individual kinetic variations for the methadone enantiomers, while the data also revealed the importance of the scaling factors used when rCYP systems are applied. Furthermore, based on the kinetic differences Chinese were predicted to be more sensitive towards methadone-induced cardiotoxicity making it even more important to replace rac-methadone by R-methadone to decrease the risk of methadone-induced cardiotoxicity in the clinical setting. Altogether, the present study shows that this PBK modeling-based NAM approach combining in vitro data and in silico modelling is promising to predict the role of kinetics in inter-ethnic and inter-individual variation in cardiotoxicity, which can be used to refine the cardiac safety evaluation in the preclinical stage.

\section{Acknowledgements}

This work was funded by a Grant from the China Scholarship Council (No. 201607720029 to MIAOYING SHI).

\section{Conflicts of interest:}

All authors declare that they have no conflict of interest.

\section{Supplementary data}

For convenience, supplementary materials can be found after reference section 


\section{Reference}

Achour B, Barber J, \& Rostami-Hodjegan A (2014). Expression of hepatic drug-metabolizing cytochrome p450 enzymes and their intercorrelations: a meta-analysis. Drug metabolism and disposition 42: 1349-1356.

Alinejad S, Kazemi T, Zamani N, Hoffman RS, \& Mehrpour O (2015). A systematic review of the cardiotoxicity of methadone. EXCLI journal 14: 577.

Ansermot N, Albayrak Ö, Schläpfer J, Crettol S, Croquette-Krokar M, Bourquin M, et al. (2010). Substitution of (R, S)-methadone by (R)-methadone: impact on QTc interval. Archives of internal medicine 170: 529-536.

Badhan RK, Gittins R, \& Al Zabit D (2019). The optimization of methadone dosing whilst treating with rifampicin: A pharmacokinetic modeling study. Drug and alcohol dependence 200: 168-180.

Barter ZE, Bayliss MK, Beaune PH, Boobis AR, Carlile DJ, Edwards RJ, et al. (2007). Scaling factors for the extrapolation of in vivo metabolic drug clearance from in vitro data: reaching a consensus on values of human micro-somal protein and hepatocellularity per gram of liver. Current drug metabolism 8: 33-45.

Barter ZE, Tucker GT, \& Rowland-Yeo K (2013). Differences in cytochrome p450-mediated pharmacokinetics between chinese and caucasian populations predicted by mechanistic physiologically based pharmacokinetic modelling. Clinical pharmacokinetics 52: 1085-1100.

Berezhkovskiy LM (2004). Determination of volume of distribution at steady state with complete consideration of the kinetics of protein and tissue binding in linear pharmacokinetics. Journal of pharmaceutical sciences 93: 364-374.

Blanchette AD, Burnett SD, Grimm FA, Rusyn I, Chiu WA (2020) A Bayesian Method for Population-wide Cardiotoxicity Hazard and Risk Characterization Using an In Vitro Human Model. Toxicological Sciences 178(2):391-403

Brown RP, Delp MD, Lindstedt SL, Rhomberg LR, \& Beliles RP (1997). Physiological parameter values for physiologically based pharmacokinetic models. Toxicology and industrial health 13: 407-484.

Burnett S D, Blanchette A D, Chiu W A, et al. (2021). Human induced pluripotent stem cell (iPSC)-derived cardiomyocytes as an in vitro model in toxicology: strengths and weaknesses for hazard identification and risk characterization. Expert Opinion on Drug Metabolism \& Toxicology

Burnett S D, Blanchette A D, Grimm F A, et al. (2019). Population-based toxicity screening in human induced pluripotent stem cell-derived cardiomyocytes[J]. Toxicology and applied pharmacology 381: 114711.

Chang Y, Fang WB, Lin SN, \& Moody DE (2011). Stereo-selective metabolism of methadone by human liver microsomes and cDNA-expressed cytochrome P450s: a reconciliation. Basic \& clinical pharmacology \& toxicology 108: 55-62.

Chen Y, Liu L, Nguyen K, \& Fretland AJ (2011). Utility of intersystem extrapolation factors in early reaction phenotyping and the quantitative extrapolation of human liver microsomal intrinsic clearance using recombinant cytochromes P450. Drug metabolism and disposition 39: 373-382.

Chiba K, Shimizu K, Kato M, Miyazaki T, Nishibayashi T, Terada K, et al. (2017). Estimation of interindividual variability of pharmacokinetics of CYP2C9 substrates in humans. Journal of Pharmaceutical Sciences 106: 26952703 .

Corning (2014). Corning UltraPool HLM 150 Characterization-CLS-DL-AN-GT-236. Retrieved from: https://www.corning.com/catalog/cls/documents/application-notes/CLS-DL-AN-GT-236_DL.pdf (Accessed: $10^{\text {th }}$ October 2020). 
Crewe H, Barter Z, Rowland Yeo K, \& Rostami-Hodjegan A (2011). Are there differences in the catalytic activity per unit enzyme of recombinantly expressed and human liver microsomal cytochrome P450 2C9? A systematic investigation into inter-system extrapolation factors. Biopharmaceutics \& drug disposition 32: 303-318.

Curtis MJ, Alexander S, Cirino G, Docherty JR, George CH, Giembycz MA, et al. (2018). Experimental design and analysis and their reporting II: Updated and simplified guidance for authors and peer reviewers. British Journal of Pharmacology, 175(7), 987-993.

Eap CB, Buclin T, \& Baumann P (2002). Interindividual variability of the clinical pharmacokinetics of methadone. Clinical pharmacokinetics 41: 1153-1193.

Eap CB, Crettol S, Rougier JS, Schläpfer J, Sintra Grilo L, Déglon JJ, et al. (2007). Stereoselective block of hERG channel by (S)-methadone and QT interval prolongation in CYP2B6 slow metabolizers. Clinical Pharmacology \& Therapeutics 81: 719-728.

Environmental Protection Agency (EPA) (2012). Benchmark Dose Techinical Guidance. Retrieved from https://www.epa.gov/sites/production/files/2015-01/documents/benchmark_dose_guidance.pdf

Ferri N, Siegl P, Corsini A, Herrmann J, Lerman A \& Benghozi R (2013). Drug attrition during pre-clinical and clinical development: understanding and managing drug-induced cardiotoxicity. Pharmacology \& therapeutics, $138(3), 470-484$.

Foster DJ, Somogyi AA, \& Bochner F (1999). Methadone N-demethylation in human liver microsomes: lack of stereoselectivity and involvement of CYP3A4. British journal of clinical pharmacology 47: 403-412.

Foster DJ, Somogyi AA, Dyer KR, White JM, \& Bochner F (2000). Steady-state pharmacokinetics of (R)-and (S)-methadone in methadone maintenance patients. British journal of clinical pharmacology 50: 427-440.

Garimella T, Wang R, Luo W-L, Wastall P, Kandoussi H, DeMicco M, et al. (2015). Assessment of drug-drug interactions between daclatasvir and methadone or buprenorphine-naloxone. Antimicrobial agents and chemotherapy 59: 5503-5510.

Gerber JG, Rhodes RJ, \& Gal J (2004). Stereoselective metabolism of methadone N-demethylation by cytochrome P4502B6 and 2C19. Chirality 16: 36-44.

Graziani M, \& Nisticò R (2015). Gender differences in pharmacokinetics and pharmacodynamics of methadone substitution therapy. Frontiers in pharmacology 6: 122 .

Grimm F A, Blanchette A, House J S, et al. (2018). A human population-based organotypic in vitro model for cardiotoxicity screening. Altex 35(4): 441.

Guan S, Huang M, Li X, Chen X, Chan E, \& Zhou S-F (2006). Intra-and inter-ethnic differences in the allele frequencies of cytochrome P450 2B6 gene in Chinese. Pharmaceutical research 23: 1983-1990.

Hsu Y-C, Chen B-G, Yang S-C, Wang Y-S, Huang S-P, Huang M-H, et al. (2013). Methadone concentrations in blood, plasma, and oral fluid determined by isotope-dilution gas chromatography-mass spectrometry. Analytical and bioanalytical chemistry 405: 3921-3928.

ICCVAM (Interagency Coordinating Committee on the Validation of Alternative Methods) 2018. A Strategic Roadmap for Establishing New Approaches to Evaluate the Safety of Chemicals and Medical Products in the United States. Available: https://ntp.niehs.nih.gov/go/iccvam-rdmp.

International Programme on Chemical Safety (IPCS) (2005). Chemical-specific adjustment factors for interspecies differences and human variability: guidance document for use of data in dose/ concentration-response assessment. WHO, Geneva. Retrieved from

https://apps.who.int/iris/bitstream/handle/10665/43294/9241546786_eng.pdf?sequence=1\&isAllowed=y 
Ito M, Kusuhara H, Ose A, Kondo T, Tanabe K, Nakayama H, et al. (2017). Pharmacokinetic modeling and Monte Carlo simulation to predict interindividual variability in human exposure to oseltamivir and its active metabolite, Ro 64-0802. The AAPS Journal 19: 286-297.

Jinno H, Tanaka-Kagawa T, Ohno A, Makino Y, Matsushima E, Hanioka N, et al. (2003). Functional characterization of cytochrome P450 2B6 allelic variants. Drug metabolism and disposition 31: 398-403.

Kandel SE, \& Lampe JN (2014). Role of protein-protein interactions in cytochrome P450-mediated drug metabolism and toxicity. Chemical research in toxicology 27: 1474-1486.

Ke AB, Nallani SC, Zhao P, Rostami-Hodjegan A, \& Unadkat JD (2014). Expansion of a PBPK model to predict disposition in pregnant women of drugs cleared via multiple CYP enzymes, including CYP2B6, CYP2C9 and CYP2C19. British journal of clinical pharmacology 77: 554-570.

Kharasch ED (2017). Current concepts in methadone metabolism and transport. Clinical pharmacology in drug development 6: 125-134.

Li J, Bluth MH (2011). Pharmacogenomics of drug metabolizing enzymes and transporters: implications for cancer therapy. Pharmacogenomics and personalized medicine 4: 11.

Li Y, Kantelip J P, Gerritsen-van Schieveen P, et al. (2008). Interindividual variability of methadone response. Molecular diagnosis \& therapy, 12(2), 109-124.

Liu P, Foster G, LaBadie R, Somoza E, \& Sharma A (2007). Pharmacokinetic interaction between voriconazole and methadone at steady state in patients on methadone therapy. Antimicrobial agents and chemotherapy 51: 110118.

Lugo RA, Satterfield KL, \& Kern SE (2005). Pharmacokinetics of methadone. Journal of Pain \& Palliative Care Pharmacotherapy 19: 13-24.

Malinowski HJ, Westelinck A, Sato J, \& Ong T (2008). Same drug, different dosing: differences in dosing for drugs approved in the United States, Europe, and Japan. The Journal of Clinical Pharmacology 48: 900-908.

Martin RL, McDermott JS, Salmen HJ, Palmatier J, Cox BF, \& Gintant GA (2004). The utility of hERG and repolarization assays in evaluating delayed cardiac repolarization: influence of multi-channel block. Journal of cardiovascular pharmacology 43: 369-379.

McGraw J, \& Waller D (2012). Cytochrome P450 variations in different ethnic populations. Expert opinion on drug metabolism \& toxicology 8: 371-382.

Mehrotra N, Tang L, Phelps SJ, \& Meibohm B (2012). Evaluation of vancomycin dosing regimens in preterm and term neonates using Monte Carlo simulations. Pharmacotherapy: The Journal of Human Pharmacology and Drug Therapy 32: 408-419.

National Health and Family Planning Commission (NHFPC) (2007a). Reference individuals for use in radiation protection-Part 1: Physique parameters. vol GBZ/T 200.1, Pub. L. No. GBZ/T 200.1. China: National Health and Family Planning Commission of the People's Republic of China. Retrieved from

http://www.nirp.cn/userfiles/file/GBZT200.1-2007.pdf.

NHFPC (2007b) Reference individuals for use in radiation protectionPart 2: masses of main organs and tissues. vol GBZ/T 200.2. National Health and Family Planning Commission of the People's Republic of China. Retrieved from http://www.nirp.cn/userfiles/file/ GBZT200.2-2007.pdf.

NHFPC (2014) Reference individiuals for use in radiation protection-Part 3: main physiological parameters. vol GBZ/T 200.3. National Health and Family Planning Commission of the People's Republic of China. Retrieved from http:/www.nirp.cn/userfiles/file/GBZT200.32014.pdf. 
Ning J, Louisse J, Spenkelink B, Wesseling S, \& Rietjens IMCM (2017). Study on inter-ethnic human differences in bioactivation and detoxification of estragole using physiologically based kinetic modeling. Archives of toxicology 91: 3093-3108.

Ning J, Rietjens IMCM, \& Strikwold M (2019). Integrating physiologically based kinetic (PBK) and Monte Carlo modelling to predict inter-individual and inter-ethnic variation in bioactivation and liver toxicity of lasiocarpine. Archives of toxicology 93: 2943-2960.

Proctor N, Tucker G, \& Rostami-Hodjegan A (2004). Predicting drug clearance from recombinantly expressed CYPs: intersystem extrapolation factors. Xenobiotica 34: 151-178.

Rietjens IMCM, Louisse J, \& Punt A (2011). Tutorial on physiologically based kinetic modeling in molecular nutrition and food research. Molecular nutrition \& food research 55: 941-956.

Shi M, Bouwmeester H, Rietjens IMCM, \& Strikwold M (2020a). Integrating in vitro data and physiologically based kinetic modeling-facilitated reverse dosimetry to predict human cardiotoxicity of methadone. Archives of Toxicology: 1-19.

Shi M, Tien NT, de Haan L, Louisse J, Rietjens IMCM, \& Bouwmeester H (2020b). Evaluation of in vitro models of stem cell-derived cardiomyocytes to screen for potential cardiotoxicity of chemicals. Toxicology in Vitro: 104891 .

Shu Y, Wang L-S, Xiao W-M, Wang W, Huang S-1, \& Zhou H-H (2000). Probing CYP2C19 and CYP3A4 activities in Chinese liver microsomes by quantification of 5-hydroxyomeprazole and omeprazole sulphone. Acta Pharmacologica Sinica 21: 753-758.

Strikwold M, Spenkelink B, Woutersen RA, Rietjens IMCM, \& Punt A (2017). Development of a combined in vitro physiologically based Kinetic (PBK) and monte carlo modelling approach to predict interindividual human variation in phenol-induced developmental toxicity. Toxicological sciences 157: 365-376.

Totah RA, Allen KE, Sheffels P, Whittington D, \& Kharasch ED (2007). Enantiomeric metabolic interactions and stereoselective human methadone metabolism. Journal of Pharmacology and Experimental Therapeutics 321: 389-399.

Totah RA, Sheffels P, Roberts T, Whittington D, Thummel K, \& Kharasch ED (2008). Role of CYP2B6 in stereoselective human methadone metabolism. Anesthesiology: The Journal of the American Society of Anesthesiologists 108: 363-374.

Tracy TS, Chaudhry AS, Prasad B, Thummel KE, Schuetz EG, Zhong X-b, et al. (2016). Interindividual variability in cytochrome P450-mediated drug metabolism. Drug Metabolism and Disposition 44: 343-351.

WFSR (2020). QIVIVE tools-Partition coefficients. [Online]. Available at: https://wfsr.shinyapps.io/wfsrqivivetools/ (Accessed: 15th September 2020).

Yamazaki H, Johnson WW, Ueng Y-F, Shimada T, \& Guengerich FP (1996). Lack of Electron Transfer from Cytochrome b5 in Stimulation of Catalytic Activities of Cytochrome P450 3A4 CHARACTERIZATION OF A RECONSTITUTED CYTOCHROME P450 3A4/NADPH-CYTOCHROME P450 REDUCTASE SYSTEM AND STUDIES WITH APO-CYTOCHROME b5. Journal of Biological Chemistry 271: 27438-27444.

Zanger UM, \& Klein K (2013). Pharmacogenetics of cytochrome P450 2B6 (CYP2B6): advances on polymorphisms, mechanisms, and clinical relevance. Frontiers in genetics 4: 24 .

Zanger UM, \& Schwab M (2013). Cytochrome P450 enzymes in drug metabolism: regulation of gene expression, enzyme activities, and impact of genetic variation. Pharmacology \& therapeutics 138: 103-141.

Zhang H, Gao N, Liu T, Fang Y, Qi B, Wen Q, et al. (2015a). Effect of cytochrome b5 content on the activity of polymorphic CYP1A2, 2B6, and 2E1 in human liver microsomes. PloS one 10: e0128547. 
Zhang H, Gao N, Tian X, Liu T, Fang Y, Zhou J, et al. (2015b). Content and activity of human liver microsomal protein and prediction of individual hepatic clearance in vivo. Scientific reports 5: 17671.

Zhang X, Tsang AM, Okino MS, Power FW, Knaak JB, Harrison LS, et al. (2007). A physiologically based pharmacokinetic/pharmacodynamic model for carbofuran in Sprague-Dawley rats using the exposure-related dose estimating model. Toxicological Sciences 100: 345-359.

Zhou Y, Ingelman-Sundberg M, \& Lauschke VM (2017). Worldwide distribution of cytochrome P450 alleles: a meta-analysis of population-scale sequencing projects. Clinical Pharmacology \& Therapeutics 102: 688-700. 


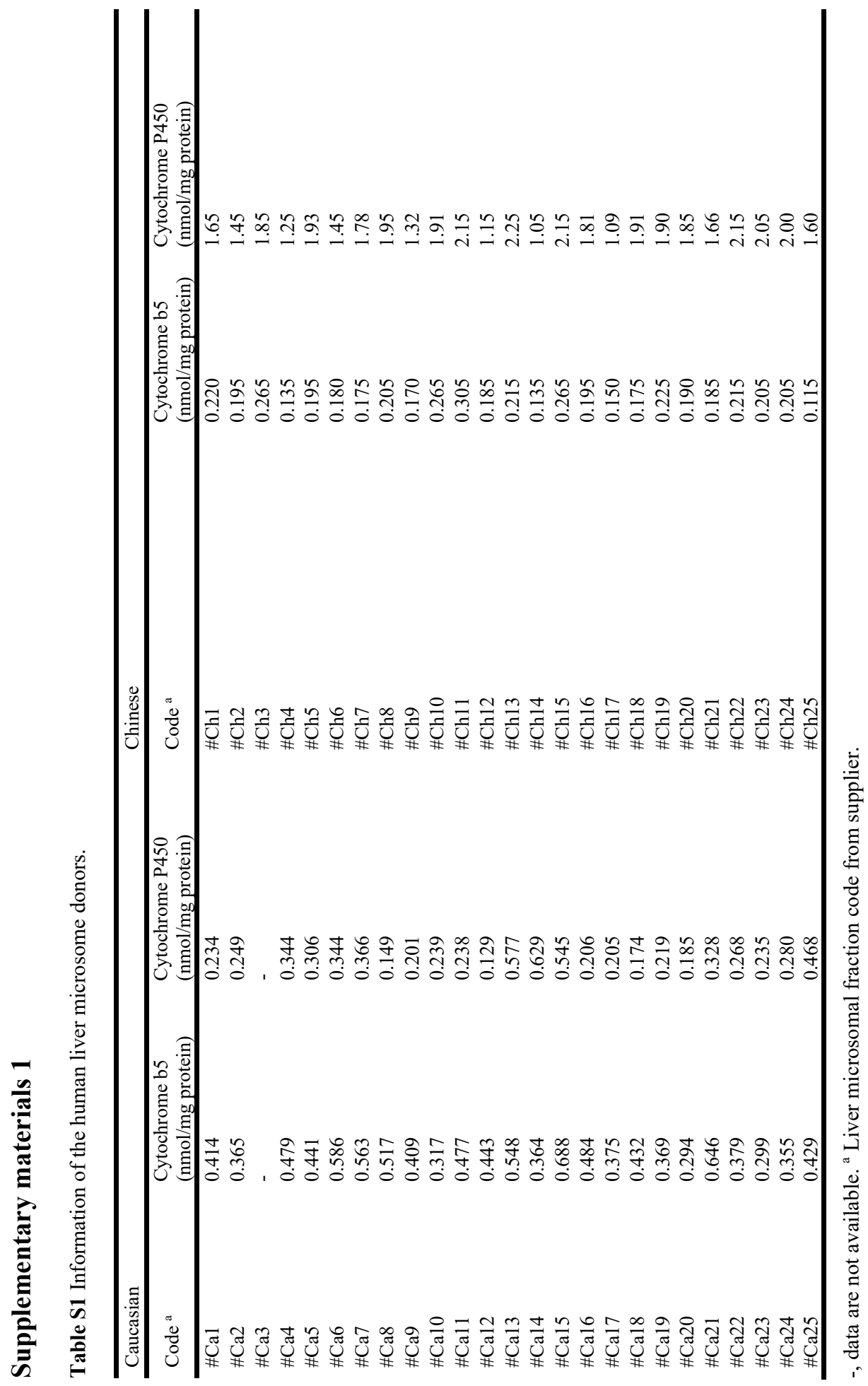




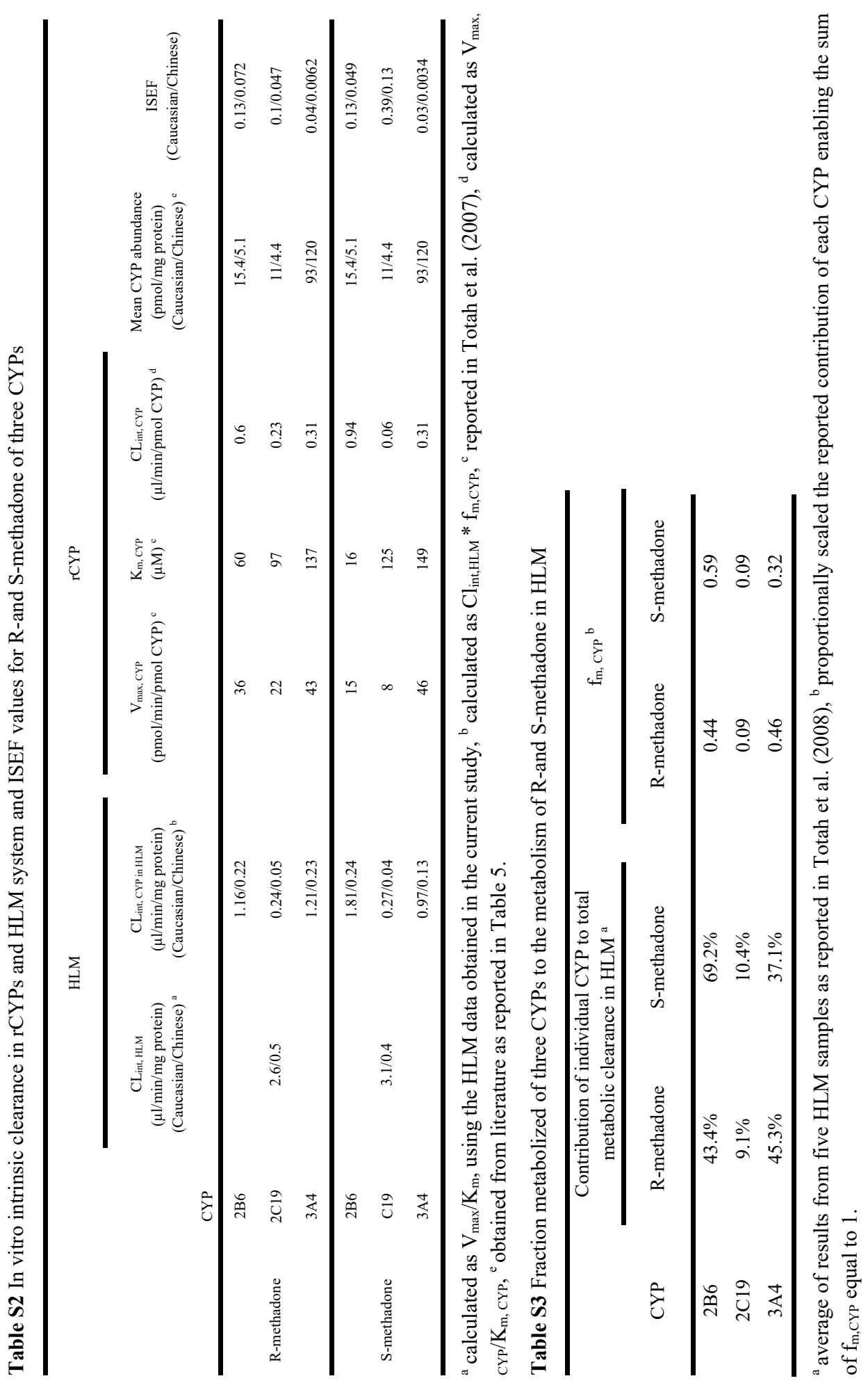




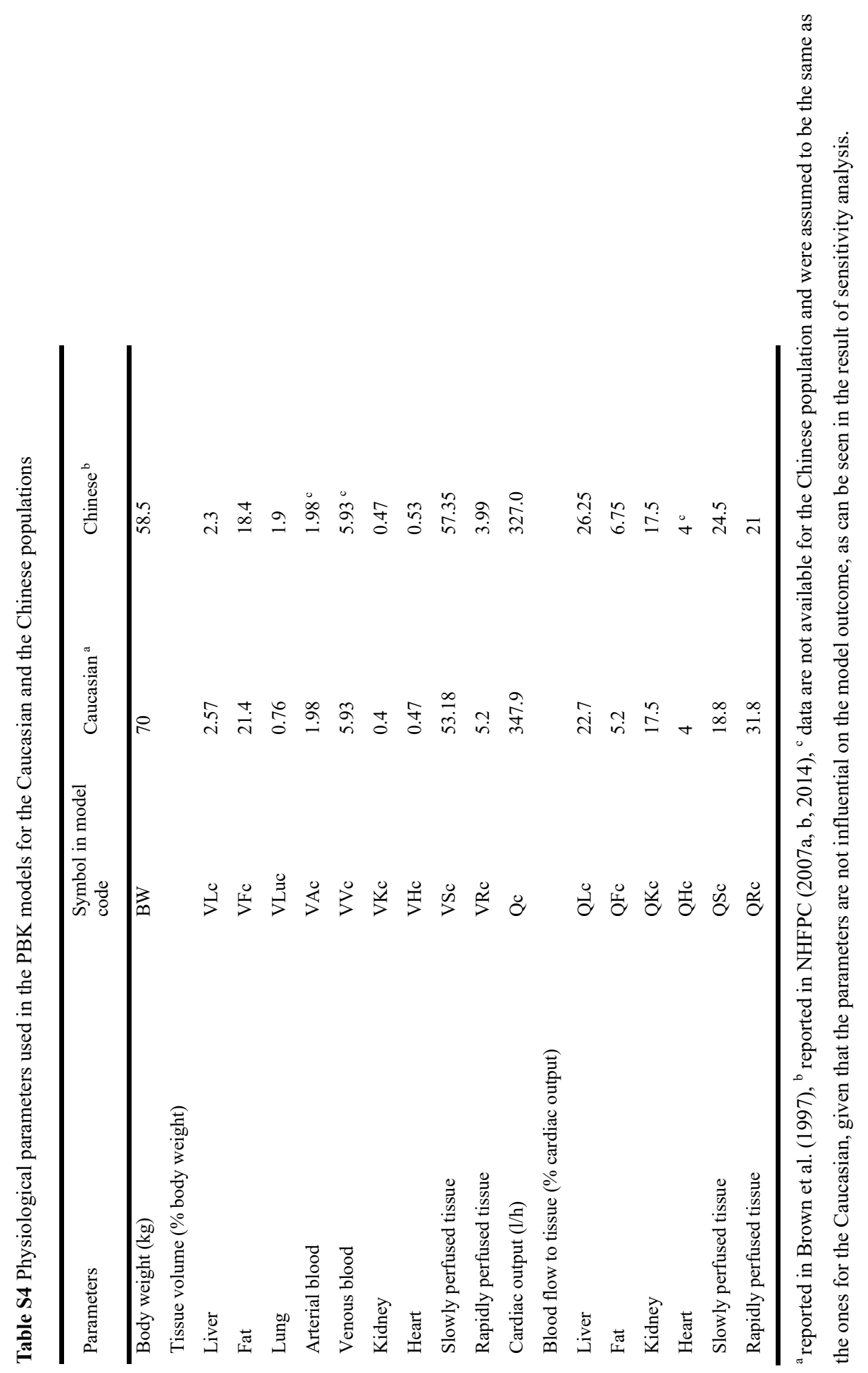




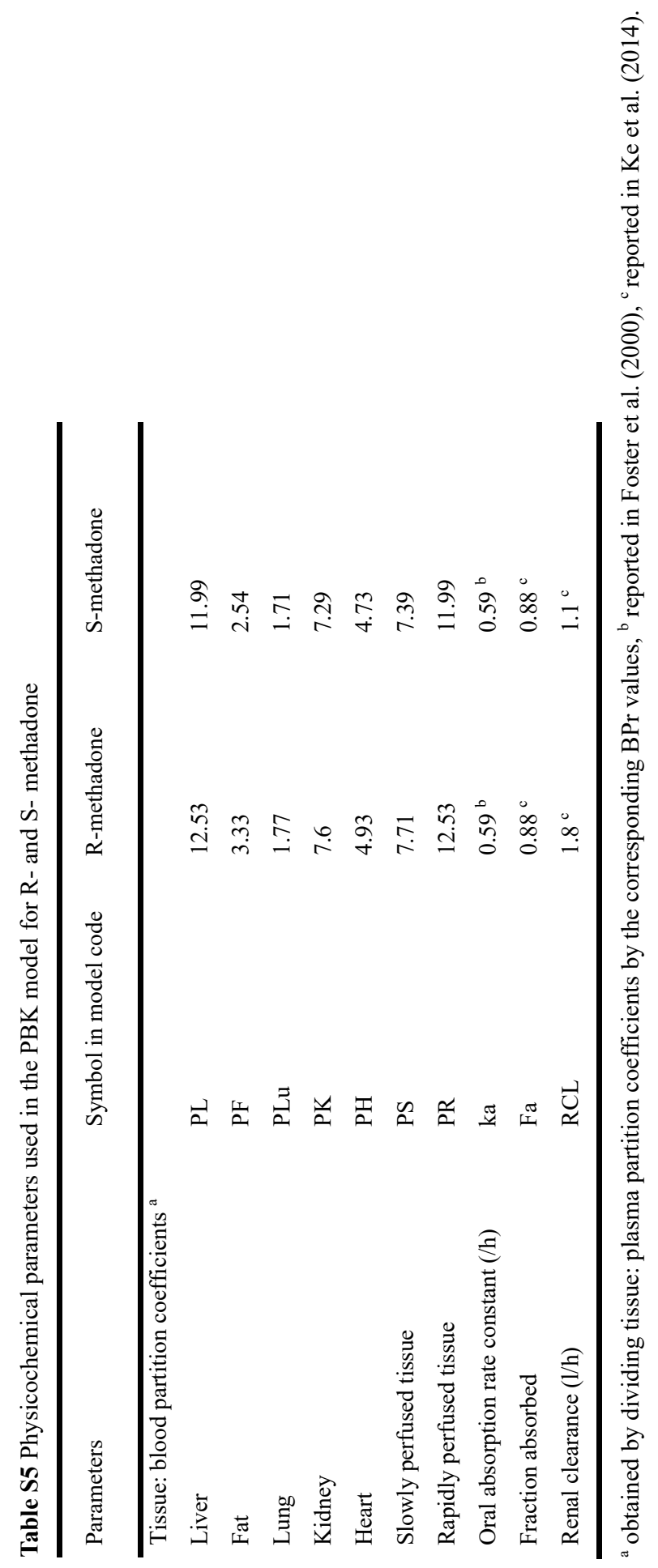




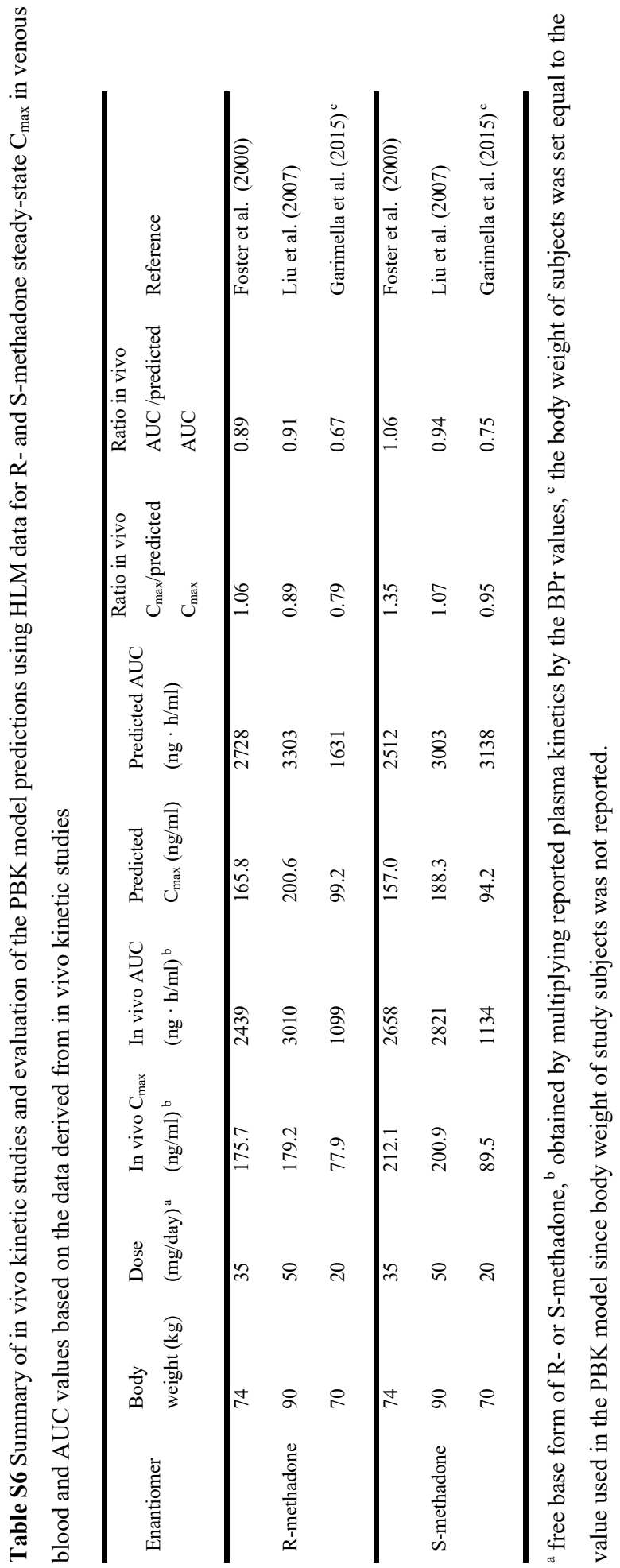




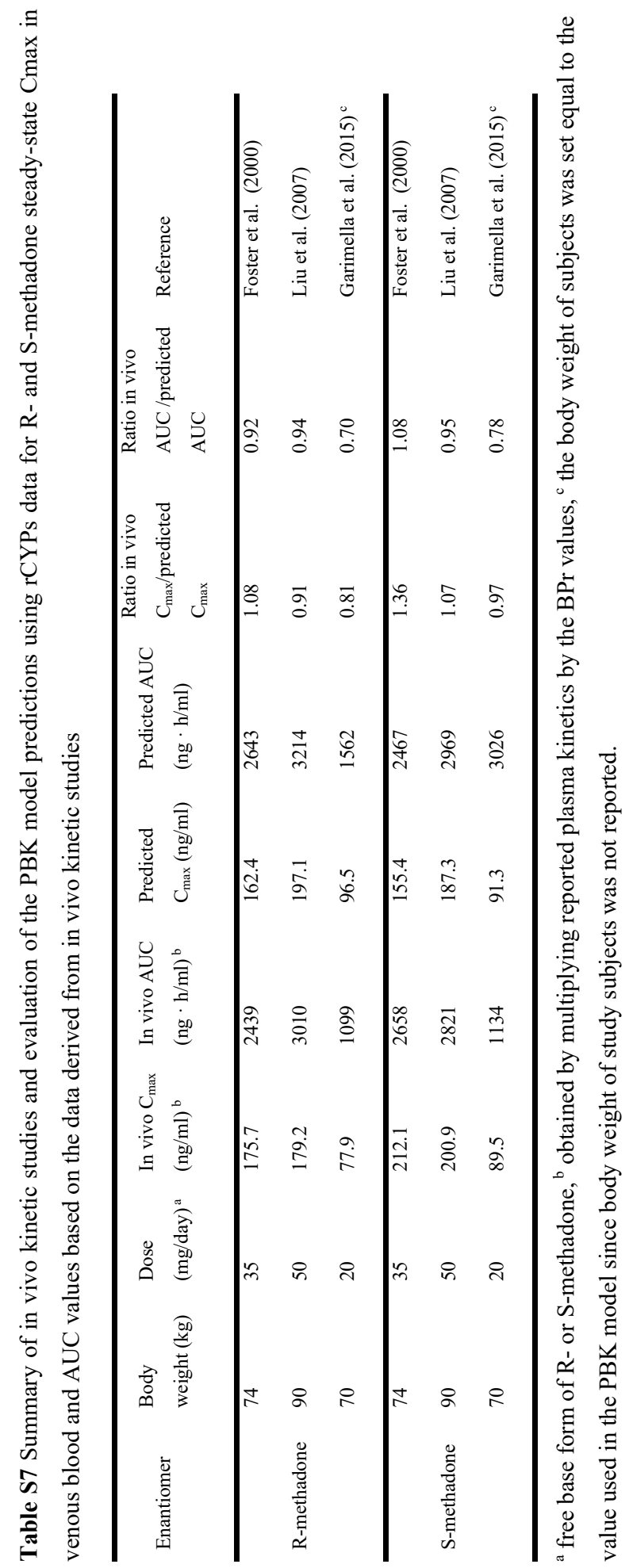




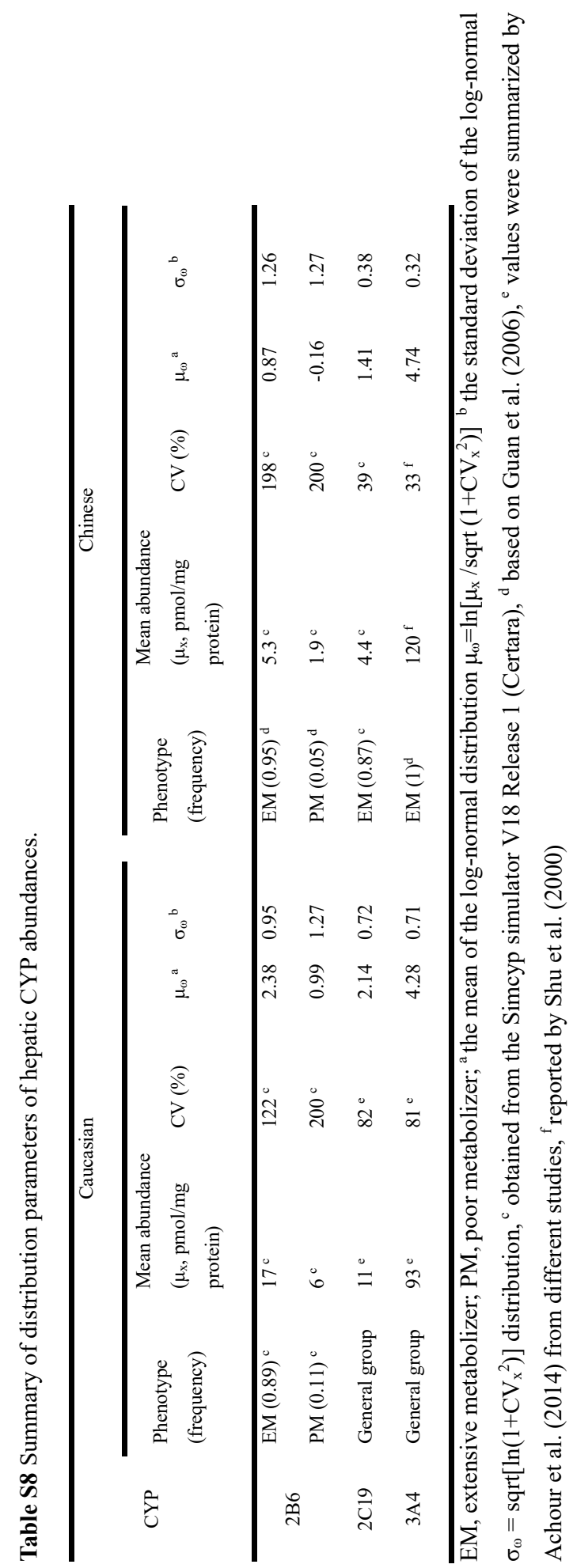




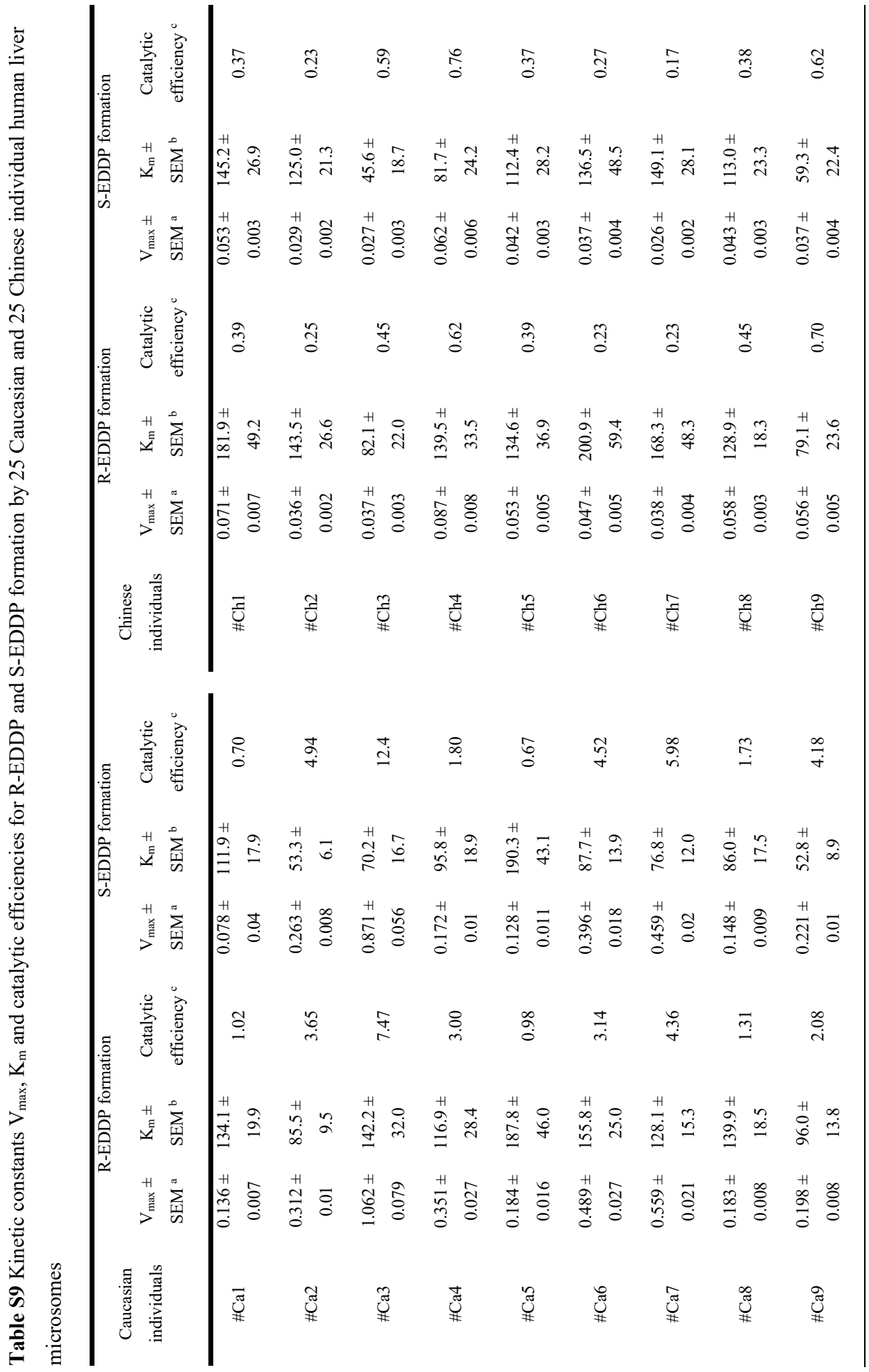




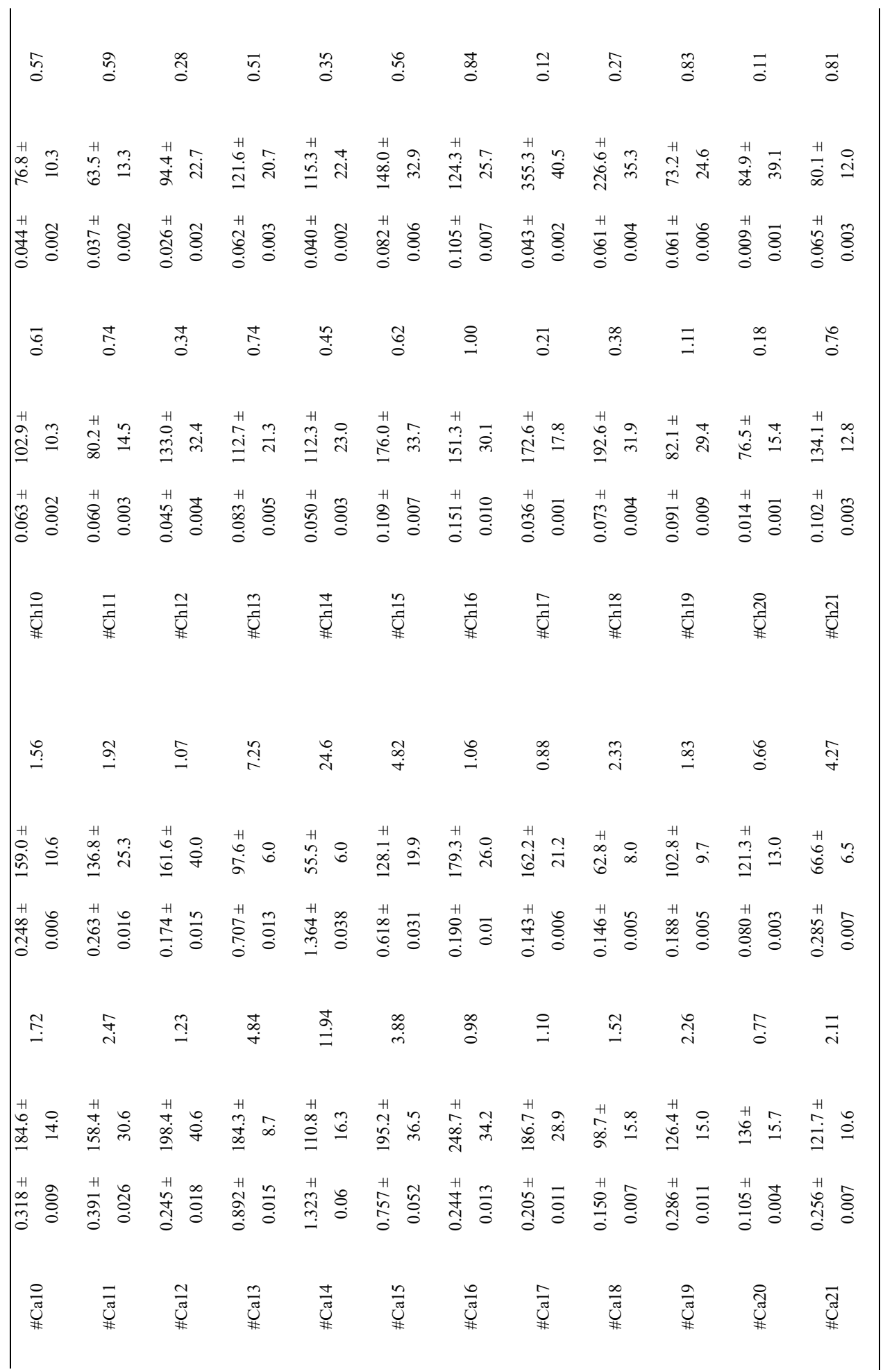




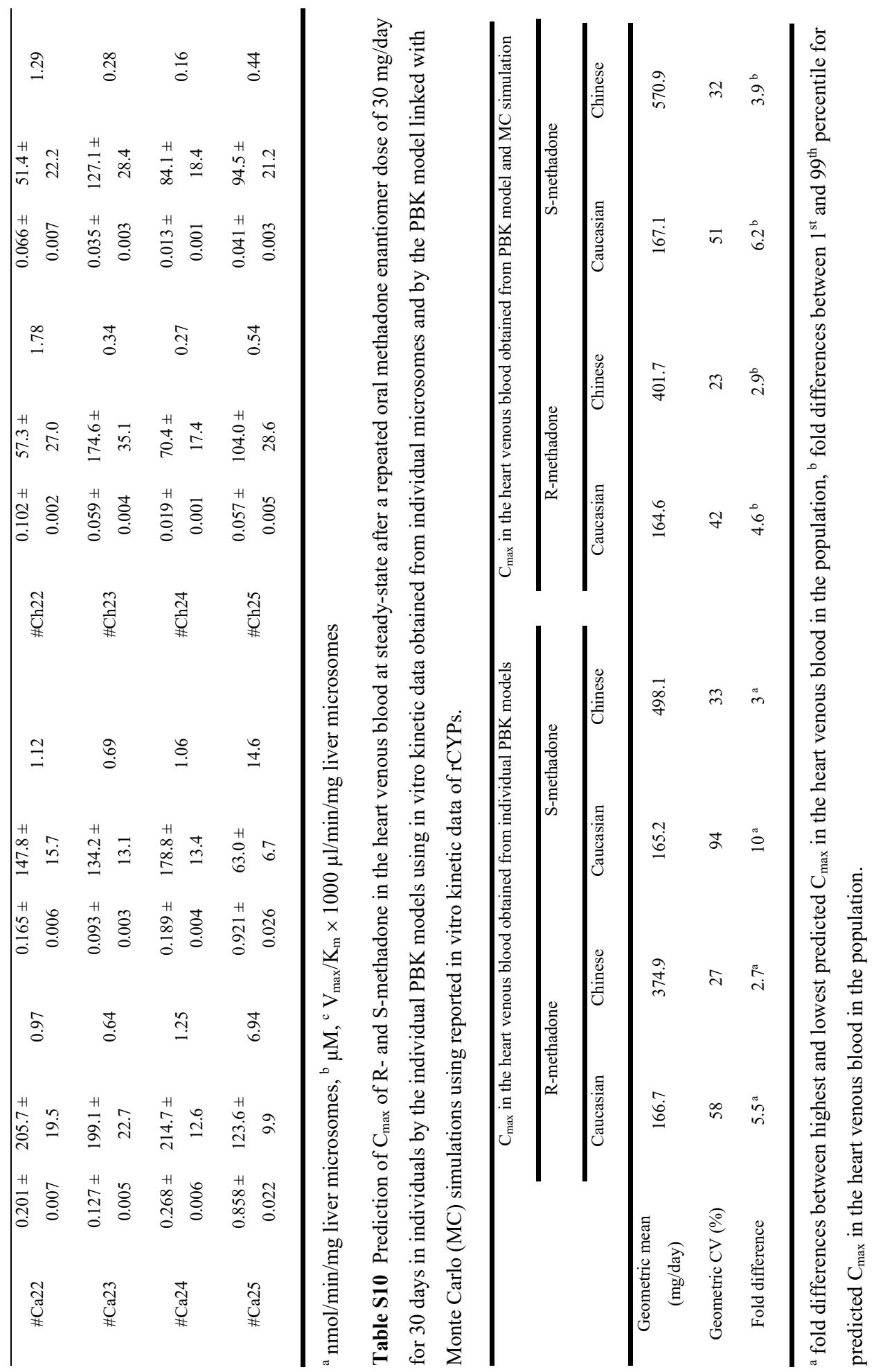



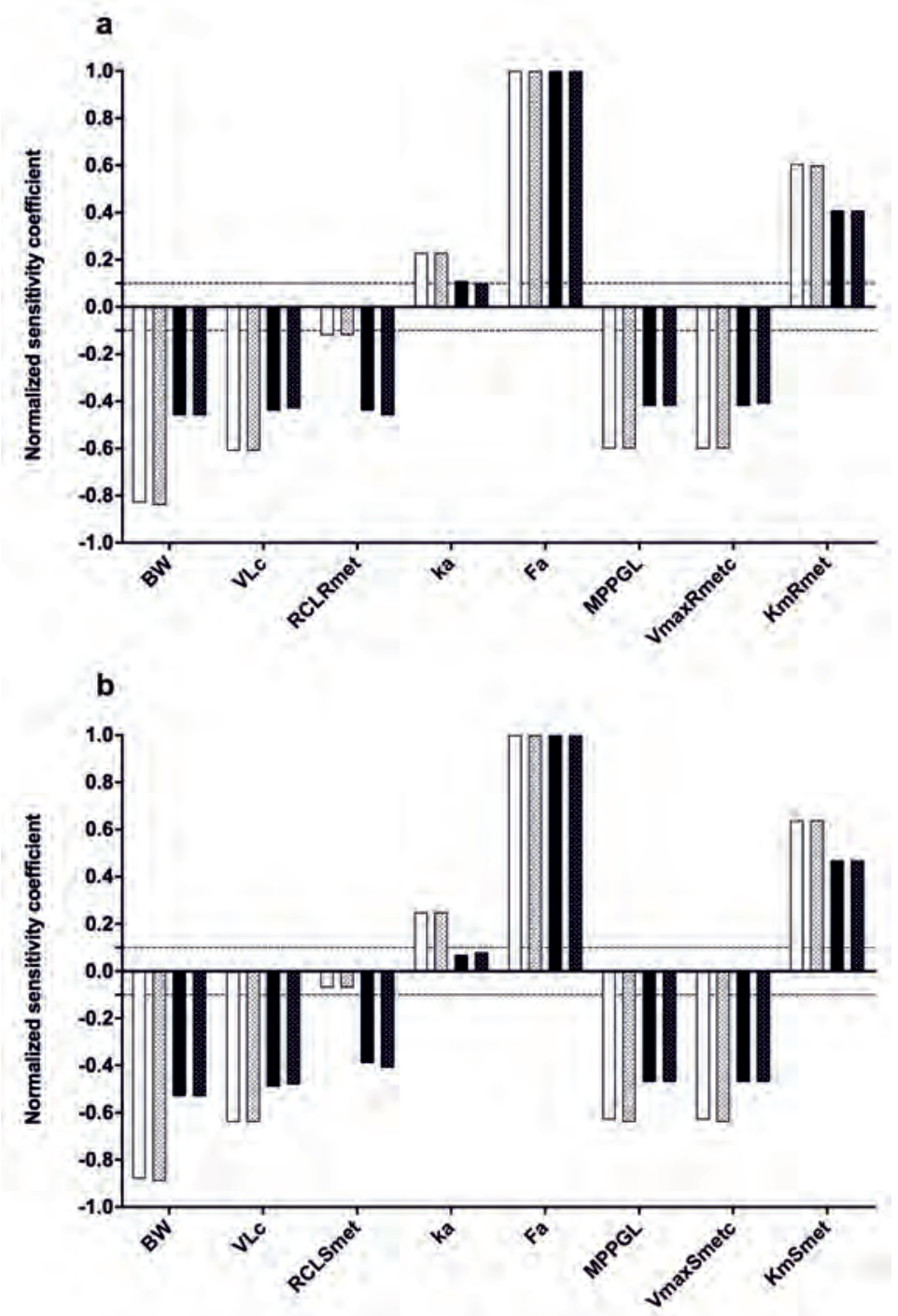

Figure S1 SCs of PBK model parameters for the prediction of steady-state $\mathrm{C}_{\max }$ of R-methadone (a) and S-methadone (b) in the heart venous blood upon the oral repeated methadone enantiomer doses of $10 \mathrm{mg}$ enantiomers/day (white bars for Caucasians, black bars for Chinese) and $100 \mathrm{mg}$ enantiomers/day (white bars with dots for Caucasians, black bars with dots for Chinese) for 30 days. BW, body weight; VLc, fraction of liver; RCLRmet and RCLSmet, renal clearance of R- and Smethadone; ka, absorption rate constant; Fa, oral fraction absorbed; MPPGL, microsomal protein per gram of liver; VmaxRmetc and VmaxSmetc, unscaled maximum rate of R- and S-methadone metabolism in liver; KmRmet and KmSmet, Michaelis-Menten constant for R- and S-methadone metabolism in liver. 

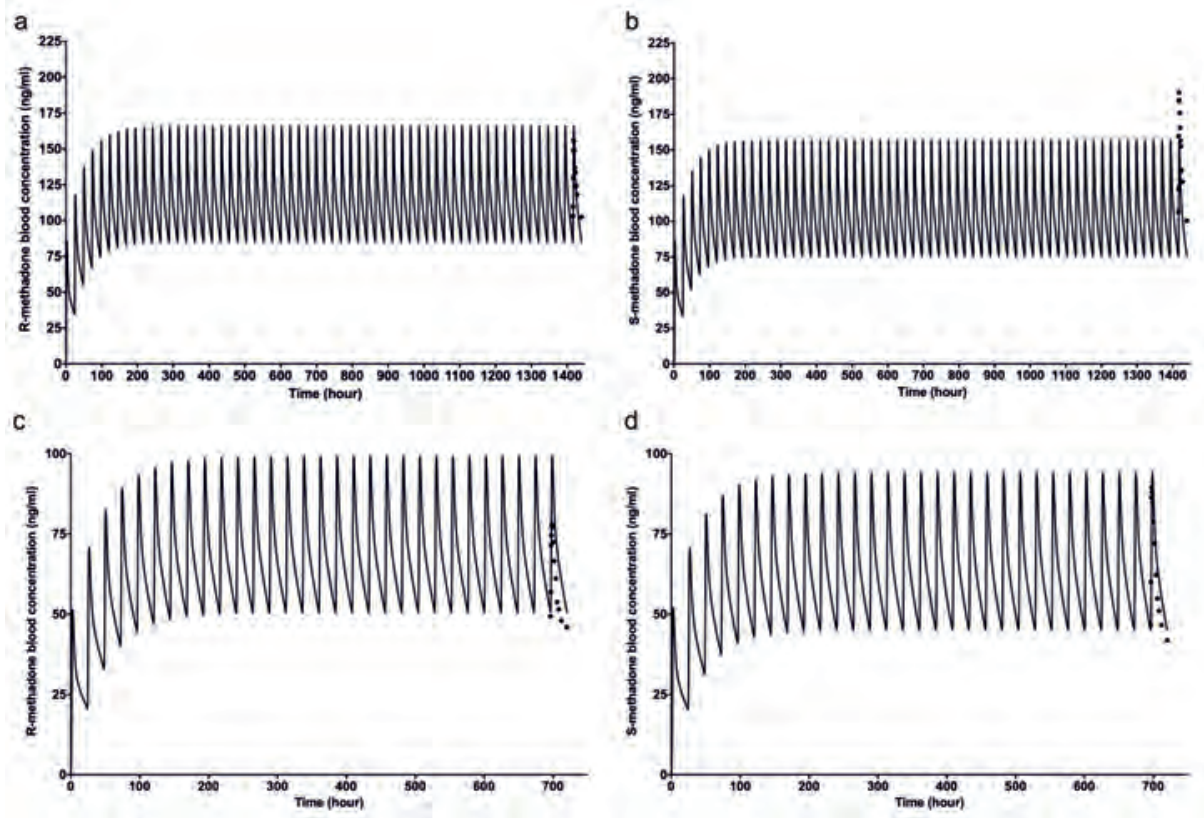

Figure S2 Blood concentration-time curves of R-methadone $(\mathbf{a}, \mathbf{c})$ and S-methadone $(\mathbf{b}, \mathbf{d})$ in human predicted with the PBK model (lines) using HLM kinetic data and published in vivo data (dots) after a repeated oral rac-methadone dose of $70 \mathrm{mg} /$ day for 60 days (a, b) (Foster et al., 2000) and $40 \mathrm{mg} /$ day for 30 days (c, d) (Garimella et al., 2015).
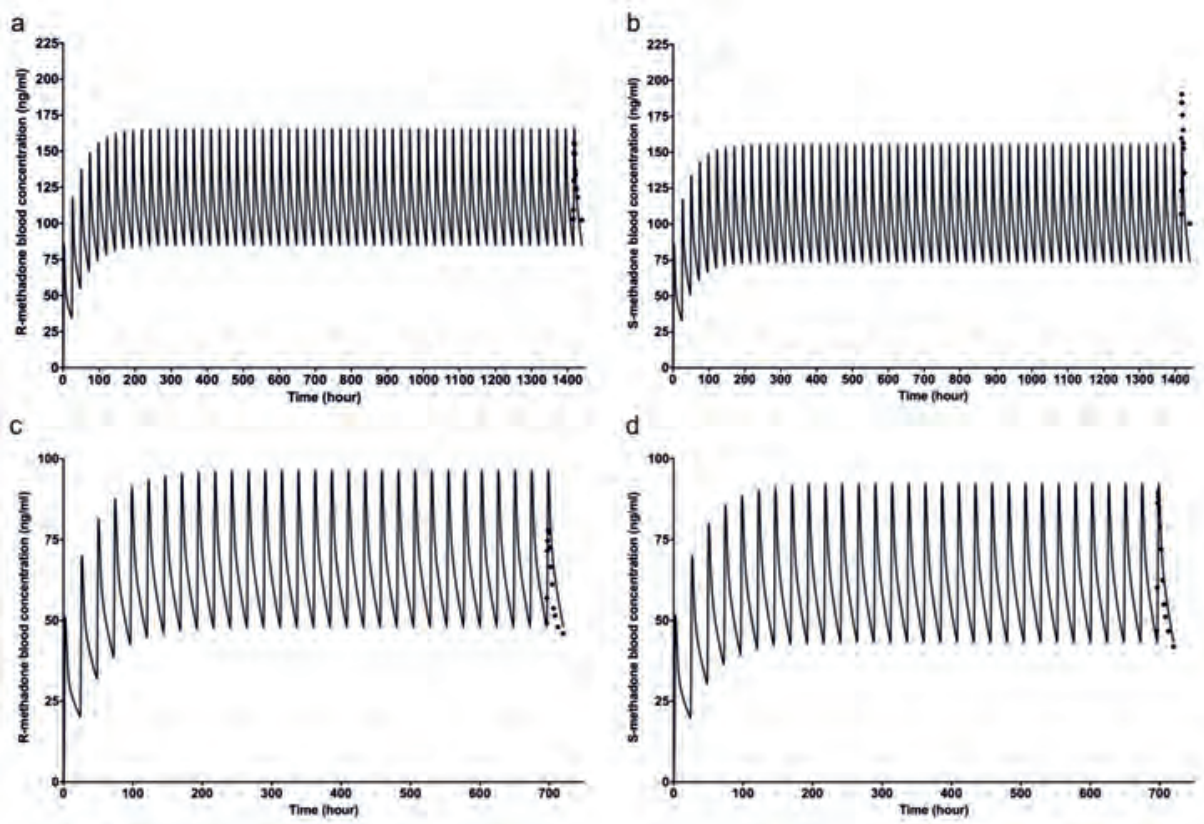
Figure S3 Blood concentration-time curves of R-methadone $(\mathbf{a}, \mathbf{c})$ and S-methadone $(\mathbf{b}, \mathbf{d})$ in human predicted with the PBK model (lines) using rCYPs kinetic data and published in vivo data (dots) after a repeated oral rac-methadone dose of $70 \mathrm{mg}$ /day for 60 days (a, b) (Foster et al., 2000) and $40 \mathrm{mg} / \mathrm{day}$ for 30 days (c, d) (Garimella et al., 2015).

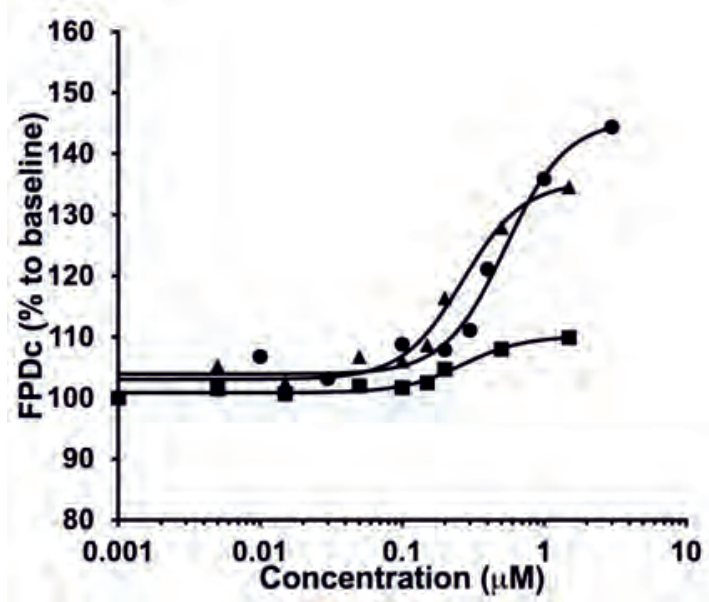

Figure S4 Concentration-response curves for the effect of rac-methadone (circles), R-methadone (squares) and S-methadone (triangles) on corrected field potential duration (FPDc) in human induced pluripotent stem cell derived cardiomyocytes detected by the multielectrode array. The concentrationresponse curve of rac-methadone (Shi et al., 2020a) was corrected to the curve of R- and S-methadone based on the potency difference between R- and S-methadone in blocking potassium channels as reported in Eap et al., (2007).
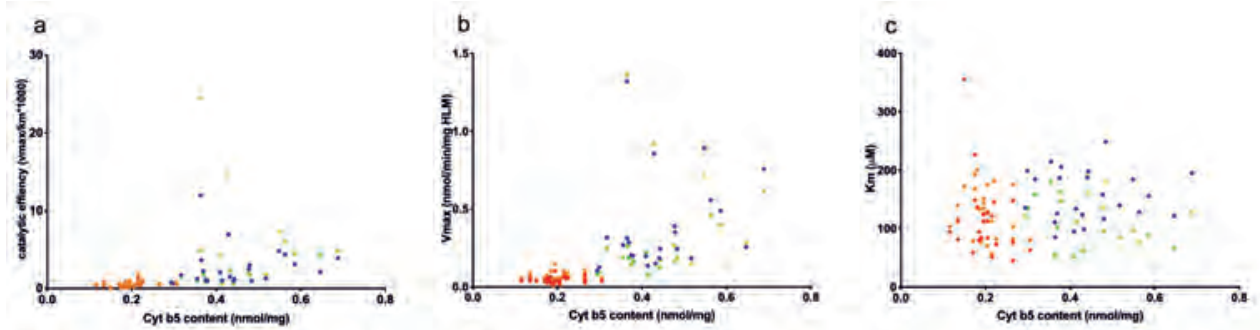

Figure S5 Correlation between Cytochrome b5 (Cyt b5) content and catalytic efficiency (a), $V_{\max }$ (b) and $\mathrm{K}_{\mathrm{m}}$ (c). Dots in blue, green, orange and red represent the data for R-methadone in Caucasians, for S-methadone in Caucasians, R-methadone in Chinese and S-methadone in Chinese, respectively. The correlation coefficients $(\mathrm{r}$ ) for the correlations are as follows: $\mathrm{r}=0.79 * * * *$ for $\mathrm{R}$-methadone; $\mathrm{r}=0.79 * * * *$ for S-methadone (a), $\mathrm{r}=0.82 * * * *$ for $\mathrm{R}$-methadone; $\mathrm{r}=0.8^{* * * *}$ for S-methadone (b) and $\mathrm{r}=0.19$ for $\mathrm{R}$ methadone; $\mathrm{r}=-0.06$ for S-methadone (c). The data distribution was checked using KolmogorovSmirnov normality test. Since most data sets were log-normally distributed, nonparametric Spearman's 
correlation was used. A p value $<0.05$ was regarded as statistically significant. The $\mathrm{r}$ values are marked with $*$ with $\mathrm{p}<0.05: *, \mathrm{p}<0.01:^{* *}, \mathrm{p}<0.001:^{* * *}$ and $\mathrm{p}<0.0001: * * *$ Statistical analysis was performed by Graph Pad Prism 8.0 (GraphPad Software Inc.).

\section{Supplementary materials 2}

Model code for Caucasian population without Monte Carlo simulation. The enantiomeric interaction equations are shown in italic.

;Model code

; Physiological parameters

; Tissue volumes ( $\mathrm{L}$ or $\mathrm{Kg}$ )

$\mathrm{BW}=70 \quad$; body weight human in $\mathrm{kg}$ (Brown et al., 1997)

; all fractions taken from Brown et al. (1997)

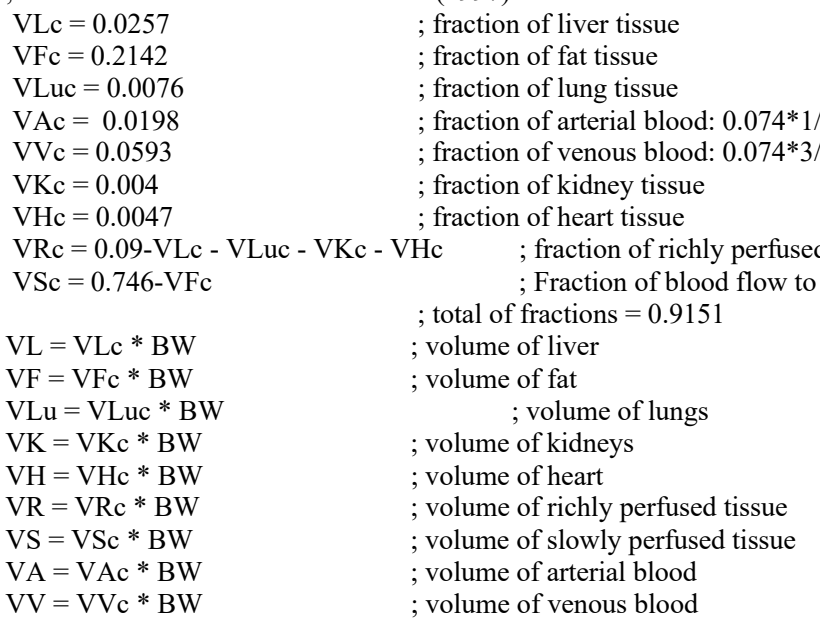

;Blood flow rates $(\mathrm{L} / \mathrm{h})$

$\mathrm{QC}=15 * \mathrm{BW}^{\wedge} 0.74 \quad ;$ Info: $\mathrm{QC}=15 * \mathrm{BW}^{\wedge} 0.74$ (Brown, 1997)

$\mathrm{QLc}=0.227 \quad$; Fraction of blood flow to liver

$\mathrm{QFc}=0.052 \quad$; Fraction of blood flow to fat

$\mathrm{QKc}=0.175 \quad$; fraction of blood flow to kidneys

$\mathrm{QHc}=0.04 \quad$; fraction of blood flow to heart

$\mathrm{QSc}=0.24-\mathrm{QFc} \quad$; Fraction of blood flow to slowly perfused tissue

$\mathrm{QRc}=0.76-\mathrm{QLc}-\mathrm{QKc}-\mathrm{QHc} \quad$; fraction of blood flow to rapidly perfused tissue

; all fractions taken from Brown 1997

$\mathrm{QL}=\mathrm{QLc} * \mathrm{QC}$

$\mathrm{QF}=\mathrm{QFc} * \mathrm{QC}$

$\mathrm{QK}=\mathrm{QKc} * \mathrm{QC}$

$\mathrm{QH}=\mathrm{QHc} * \mathrm{QC}$

$\mathrm{QR}=\mathrm{QRc} * \mathrm{QC}$

$\mathrm{QS}=\mathrm{QSc} * \mathrm{QC}$

total of fractions $=1$

$;=============$
; Partition Coefficients

; R-Methadone

PLRmet $=12.53$

PFRmet $=3.33$

; blood flow rate to liver in $\mathrm{L} / \mathrm{hr}$

; blood flow rate to fat

; blood flow rate to kidneys

; blood flow rate to heart

; blood flow rate to richly perfused tissue

; blood flow rate to slowly perfused tissue

$$
\text { PFRmet }=3.33
$$

; liver/blood partition coefficient R-Methadone

; fat/blood partition coefficient R-Methadone 


$$
\begin{aligned}
& \text { PRRmet }=12.53 \\
& \text { PSRmet }=7.71 \\
& \text { PLuRmet }=1.77 \\
& \text { PKRmet }=7.6 \\
& \text { PHRmet }=4.93 \\
& ; \text { S-Methadone } \\
& \text { PLSmet }=11.99 \\
& \text { PFSmet }=2.54 \\
& \text { PRSmet }=11.99 \\
& \text { PSSmet }=7.39 \\
& \text { PLuSmet }=1.71 \\
& \text { PKSmet }=7.29 \\
& \text { PHSmet }=4.73
\end{aligned}
$$

; richly perfused tissues/blood partition coefficient R-Methadone

; slowly perfused tissues/blood partition coefficient R-Methadone

; lung/blood partition coefficient R-Methadone

; kidney/blood partition coefficient R-Methadone

; heart/blood partition coefficient R-Methadone

; liver/blood partition coefficient S-Methadone

; fat/blood partition coefficient S-Methadone

; richly perfused tissues/blood partition coefficient S-Methadone

; slowly perfused tissues/blood partition coefficient S-Methadone

; lung/blood partition coefficient S-Methadone

; kidney/blood partition coefficient S-Methadone

; heart/blood partition coefficient S-Methadone

$;==-==-$
; Biochemical parameters

;Linear uptake rate $(/ \mathrm{h})$

$\mathrm{ka}=0.59$

; obtained from Foster et al. (2000); Wolff et al. (2000)

;Fraction absorbed

$\mathrm{Fa}=0.88$

; obtained from Ke et al. (2013)

;Renal clearance $(\mathrm{L} / \mathrm{h})$

RCLRmet $=1.8$

Foster et al. (2000)

RCLSmet $=1.1$

; average values obtained from Boulton et al. (2001); Kharasch et al. (2009)

Foster et al. (2000)

; average values obtained from Boulton et al. (2001); Kharasch et al. (2009)

;Metabolism liver

;Scaling factors

ISEFCYP2B6R $=0.13$

ISEFCYP3A4R $=0.04$

, corrected based on Totah et al. (2007) (2008)

ISEFCYP2C19R $=0.1$

; corrected based on Totah et al. (2007) (2008)

; corrected based on Totah et al. (2007) (2008)

ISEFCYP2B $6 \mathrm{~S}=0.13$

ISEFCYP3A $4 \mathrm{~S}=0.03$

ISEFCYP2C19S $=0.39$

; corrected based on Totah et al. (2007) (2008)

; corrected based on Totah et al. (2007) (2008)

; corrected based on Totah et al. (2007) (2008)

$\mathrm{aCYP} 2 \mathrm{~B} 6=17$

; $\mathrm{aCYP} 2 \mathrm{~B} 6=6$

aCYP3A4 $=93$

$\mathrm{n}=713$

aCYP2C19 = 11

$\mathrm{n}=76$;

; EM CYP abundance level pmol/mg CYPisoform from Barter et al. (2013)

; PM CYP abundance level pmol/mg CYPisoform from Barter et al. (2013)

; CYP abundance level pmol/mg CYPisoform from Achour et al. (2014)

; CYP abundance level pmol/mg CYPisoform from Achour et al. (2014),

$\mathrm{MPL}=32$

$\mathrm{L}=\mathrm{VLc} * 1000$

; liver microsomal protein yield (mg/gram liver) (Barter et al., 2007)

; liver $=25.7($ gram $/ \mathrm{kg} \mathrm{BW})$

; in vitro recombinant incubation of R-methadone ( $\mathrm{pmol} / \mathrm{min} / \mathrm{nmol} \mathrm{CYP}$ )

VmaxRmetCYP2B6m $=36$

; Totah et al. (2007)

VmaxRmetCYP3A4m $=43$

; Totah et al. (2007)

VmaxRmetCYP2C19m $=22$

; Totah et al. (2007)

;metabolites of R-methadone, unscaled maximum rate of metabolism ( $\mathrm{pmol} / \mathrm{mg}$ protein $/ \mathrm{min}$ )

VmaxRmetCYP2B6c $=$ VmaxRmetCYP2B6m*ISEFCYP2B6R*aCYP2B6

VmaxRmetCYP3A4c $=$ VmaxRmetCYP3A4m*ISEFCYP3A4R*aCYP3A4

VmaxRmetCYP2C19c $=$ VmaxRmetCYP2C19m*ISEFCYP2C19R*aCYP2C19

;metabolites of R-Methadone, scaled maximum rate of metabolism ( $\mu \mathrm{mol} / \mathrm{h})$

VMaxRmetCYP2B6 = VmaxRmetCYP2B6c $/ 1000000 * 60 * \mathrm{MPL} * \mathrm{~L} * \mathrm{BW}$ 
VMaxRmetCYP3A4 $=$ VmaxRmetCYP3A4c $/ 1000000 * 60 * \mathrm{MPL} * \mathrm{~L} * \mathrm{BW}$

VMaxRmetCYP2C19=VmaxRmetCYP2C19c/1000000*60* MPL * L * BW

;metabolites of R-methadone, affinity constants $(\mu \mathrm{mol} / \mathrm{L})$

KmRmetCYP2B6 $=60$

$\mathrm{KmRmetCYP3A4}=137$

KmRmetCYP2C19 = 97

; in vitro recombinant incubation of S-methadone ( $\mathrm{pmol} / \mathrm{min} / \mathrm{nmol} \mathrm{CYP}$ )

VmaxSmetCYP2B6m $=15$

; Totah et al. (2007)

VmaxSmetCYP3A4m $=46$

; Totah et al. (2007)

VmaxSmetCYP2C19m $=8$

; Totah et al. (2007)

;metabolites of S-methadone, unscaled maximum rate of metabolism ( $\mathrm{pmol} / \mathrm{mg}$ protein $/ \mathrm{min}$ )

VmaxSmetCYP2B6c $=$ VmaxSmetCYP2B6m*ISEFCYP2B6S*aCYP2B6

VmaxSmetCYP3A4c $=$ VmaxSmetCYP3A4m*ISEFCYP3A4S*aCYP3A4

VmaxSmetCYP2C19c = VmaxSmetCYP2C19m*ISEFCYP2C19S*aCYP2C19

; metabolites of S-methadone, scaled maximum rate of metabolism $(\mu \mathrm{mol} / \mathrm{h})$

VMaxSmetCYP2B6 = VmaxSmetCYP2B6c $/ 1000000 * 60 * \mathrm{MPL} * \mathrm{~L} * \mathrm{BW}$

VMaxSmetCYP3A4 $=$ VmaxSmetCYP3A4c $/ 1000000 * 60 * \mathrm{MPL} * \mathrm{~L} * \mathrm{BW}$

VMaxSmetCYP2C19 $=$ VmaxSmetCYP2C19c $/ 1000000 * 60 * \mathrm{MPL} * \mathrm{~L} * \mathrm{BW}$

;metabolites of S-methadone, affinity constants (umol/L)

KmSmetCYP2B6 $=16$

$\mathrm{KmSmetCYP3A4}=149$

$\mathrm{KmSmetCYP2C} 19=125$

;Run settings

;molecular weight $(\mathrm{g} / \mathrm{mol})$

MWRmet $=309.4$

MWSmet $=309.4$

; molecular weight

; molecular weight

; R-methadone Given dose ( $\mathrm{mg} / \mathrm{kg} \mathrm{bw}$ ) and oral dose in $\mu \mathrm{mol} / \mathrm{kg}$ bw

TDOSERmet $=30$

; whole body total dose in $\mathrm{mg}$

GDOSERmet $=$ TDOSERmet $/ \mathrm{BW}$; given dose in mg per kg bw

ODOSERmet $=$ GDOSERmet $* 1 \mathrm{e}-3 /$ MWRmet $* 1 \mathrm{e} 6 \quad ;$ determine odose $(\mu \mathrm{mol} / \mathrm{kg} \mathrm{bw})$

DOSERmet $=$ ODOSERmet $*$ BW ; determine dose in $\mu$ mol

; S-methadone Given dose ( $\mathrm{mg} / \mathrm{kg} \mathrm{bw})$ and oral dose in $\mu \mathrm{mol} / \mathrm{kg}$ bw

TDOSESmet $=30 \quad$; whole body total dose in $\mathrm{mg}$

GDOSESmet $=$ TDOSESmet $/ \mathrm{BW}$; given dose in $\mathrm{mg}$ per $\mathrm{kg}$ bw

ODOSESmet $=$ GDOSESmet $* 1 \mathrm{e}-3 /$ MWSmet $* 1 \mathrm{e} 6 ;$ determine odose $(\mu \mathrm{mol} / \mathrm{kg} \mathrm{bw})$

DOSESmet $=$ ODOSESmet $*$ BW $\quad$; determine dose in $\mu \mathrm{mol}$

;Time

dose_int $=24$

; dosing interval in hours

Starttime $=0$

; in hrs

Stoptime $=30 * 24$

; in hrs (days * hours in a day)

DTMIN $=1 \mathrm{e}-6$

DTMAX $=1$

DTOUT $=0$

TOLERANCE $=0.00001$

;Kinetics

;slowly perfused tissue compartment

;ASRmet $=$ Amount R-methadone in slowly perfused tissue $(\mu \mathrm{mol})$

ASRmet' $=$ QS * (CARmet - CVSRmet $)$

Init ASRmet $=0$ 
CSRmet $=$ ASRmet $/ \mathrm{VS}$

CVSRmet $=$ CSRmet $/$ PSRmet

;ASSmet $=$ Amount S-methadone in slowly perfused tissue $(\mu \mathrm{mol})$

ASSmet' $=$ QS * (CASmet - CVSSmet)

Init ASSmet $=0$

CSSmet $=$ ASSmet $/$ VS

CVSSmet $=$ CSSmet $/$ PSSmet

; rapid perfused tissue compartment

;ARRmet $=$ Amount R-methadone in richly perfused tissue $(\mu \mathrm{mol})$

ARRmet' $=\mathrm{QR} *($ CARmet - CVRRmet $)$

Init ARRmet $=0$

CRRmet $=$ ARRmet $/ \mathrm{VR}$

CVRRmet $=$ CRRmet $/$ PRRmet

;ARSmet $=$ Amount S-methadone in richly perfused tissue $(\mu \mathrm{mol})$

ARSmet' $=\mathrm{QR} *($ CASmet - CVRSmet $)$

Init ARSmet $=0$

CRSmet $=$ ARSmet $/$ VR

CVRSmet $=$ CRSmet $/$ PRSmet

;fat compartment

;AFRmet $=$ Amount R-methadone in fat tissue $(\mu \mathrm{mol})$

AFRmet' $=\mathrm{QF} *(\mathrm{CARmet}-\mathrm{CVFRmet})$

Init AFRmet $=0$

CFRmet $=$ AFRmet $/$ VF

CVFRmet $=$ CFRmet $/$ PFRmet

;AFSmet $=$ Amount S-methadone in fat tissue $(\mu \mathrm{mol})$

AFSmet' $=\mathrm{QF} *($ CASmet - CVFSmet $)$

Init AFSmet $=0$

CFSmet $=$ AFSmet $/ \mathrm{VF}$

CVFSmet $=$ CFSmet $/$ PFSmet

; uptake methadone from GI tract

;AGIRmet $=$ Amount R-methadone remaining in GI tract $(\mu \mathrm{mol})$

Init AGIRmet $=0$

AGIRmet' $=$ pulse $($ DOSERmet $*$ Fa, 0 , dose int $)+$ AGIRmet $*-$ Ka

;AGISmet $=$ Amount S-methadone remaining in GI tract $(\mu \mathrm{mol})$

Init AGISmet $=0$

AGISmet' $=$ pulse $\left(\right.$ DOSESmet $^{*}$ Fa, 0, dose_int $)+$ AGISmet $*$-Ka

;liver compartment

;ALRmet $=$ Amount R-methadone in liver tissue $(\mu \mathrm{mol})$

ALRmet' $=\mathrm{QL} *($ CARmet - CVLRmet $)+($ AGIRmet $*$ Ka $)-$ AMLRmetCYP2B6' -AMLRmetCYP3A4' -

AMLRmetCYP2C19'

Init ALRmet $=0$

CLRmet $=$ ALRmet $/ \mathrm{VL}$

CVLRmet $=$ CLRmet $/$ PLRmet

;metabolism described by Michaelis-Menten Kinetics

;AMLRmetCYP2B6=Amount R-methadone metabolized in liver to R-EDDP by CYP2B6

;AMLRmetCYP2B6' = (VmaxRmetCYP2B6*CVLRmet $) /($ KmRmetCYP2B6 + CVLRmet $)$

;init AMLRmetCYP2B6 = 0

;AMLRmetCYP3A4=Amount R-methadone metabolized in liver to R-EDDP by CYP3A4

;AMLRmetCYP3A4' = (VmaxRmetCYP3A4*CVLRmet $) /($ KmRmetCYP3A4 + CVLRmet $)$ 
;init AMLRMetCYP3A4 = 0

;AMLRmetCYP2C19=Amount R-methadone metabolized in liver to R-EDDP by CYP2C19 ;AMLRmetCYP2C19' = (VmaxRmetCYP2C19*CVLRmet $) /($ KmRmetCYP2C19 + CVLRmet $)$ ;init AMLRMetCYP2C19 = 0

;metabolism described by enantiomeric interactions equations

AMLRmetCYP2B6'=VmaxRmetCYP2B6*((CVLRmet ${ }^{*} C V L R m e t /($ ahCYP2B6*KmRmetCYP2B6*KmRm etCYP2B6) $)+(C V L R m e t / K m R m e t C Y P 2 B 6)+\left(C V L R m e t * C V L S m e t /\left(b h C Y P 2 B 6^{*} K m R m e t C Y P 2 B 6 * K m S\right.\right.$ metCYP2B6)) $) /\left(1+\left(C V L R m e t * C V L R m e t /\left(a h C Y P 2 B 6 * K m R m e t C Y P 2 B 6{ }^{*}\right.\right.\right.$ KmRmetCYP2B6) $)+(2 * C V L R$ met/KmRmetCYP2B6)+(CVLSmet $\left.{ }^{*} C V L S m e t /(a h C Y P 2 B 6 * K m S m e t C Y P 2 B 6 * K m S m e t C Y P 2 B 6)\right)+(2 * C$ VLSmet/KmSmetCYP2B6)+(2*CVLRmet ${ }^{*}$ CVLSmet/(bhCYP2B6*KmRmetCYP2B6*KmSmetCYP2B6)))

$a h C Y P 2 B 6=5$

; homotropic interaction factor (Totah et al., 2007)

bhCYP $2 B 6=7$

; heterotropic interaction factor (Totah et al., 2007)

init AMLRmetCYP2B6 $=0$

AMLRmetCYP3A4' $=$

(VmaxRmetCYP3A4*((CVLRmet *CVLRmet/(ahCYP3A4*KmRmetCYP3A4*KmRmetCYP3A4))+(CVL Rmet/KmRmetCYP3A4)))/(1+(CVLRmet *CVLRmet/(ahCYP3A4*KmRmetCYP3A4*KmRmetCYP3A4)) $+(2 *$ CVLRmet/KmRmetCYP3A4) $+(C V L S m e t * C V L S m e t /(a h C Y P 3 A 4 * K m S m e t C Y P 3 A 4 * K m S m e t C Y P 3$ A4) $)+(2 *$ CVLSmet/KmSmetCYP3A4 $)+(2 *$ CVLRmet $* C V L S m e t /(b h C Y P 3 A 4 * K m R m e t C Y P 3 A 4 * K m S m e t$ CYP3A4)))

ahCYP $3 A 4=4$

; homotropic interaction factor (Totah et al., 2007)

bhCYP $3 A 4=2$ ; heterotropic interaction factor (Totah et al., 2007)

init AMLRmetCYP3A4 =0

AMLRmetCYP2C19' =

(VmaxRmetCYP2C19*(CVLRmet/KmRmetCYP2C19))/(1+(CVLRmet*CVLRmet/(ahCYP2C19*KmRme tCYP2C19*KmRmetCYP2C19)) +(2*CVLRmet/KmRmetCYP2C19) $+(C V L S m e t * C V L S m e t /(a h C Y P 2 C 1$ $9 *$ KmSmetCYP2C19*KmSmetCYP2C19)) + $2 *$ CVLSmet/KmSmetCYP2C19) $+(2 *$ CVLRmet $*$ CVLSmet/( bhCYP2C19*KmRmetCYP2C19*KmSmetCYP2C19)))

ahCYP2C19=42

bhCYP2C19=3

init AMLRmetCYP2C19=0
; homotropic interaction factor (Totah et al., 2007)

; heterotropic interaction factor (Totah et al., 2007)

;S-methadone

;ALSmet $=$ Amount S-methadone in liver tissue $(\mu \mathrm{mol})$

ALSmet' $=$ QL * (CASmet - CVLSmet $)+($ AGISmet * Ka) - AMLSmetCYP2B6' -AMLSmetCYP3A4' AMLSmetCYP2C19'

Init ALSmet $=0$

CLSmet $=$ ALSmet $/$ VL

CVLSmet $=$ CLSmet $/$ PLSmet

;metabolism described by Michaelis-Menten Kinetics

;AMLSmetCYP2B6=Amount Smet metabolized in liver to S-EDDP by CYP2B6

;AMLSmetCYP2B6' = (VmaxSmetCYP2B6*CVLSmet $) /($ KmSmetCYP2B6 + CVLSmet $)$

;init AMLSmetCYP2B6 $=0$

;AMLSmetCYP3A4=Amount Smet metabolized in liver to S-EDDP by CYP3A4

;AMLSmetCYP3A4' = (VmaxSmetCYP3A4*CVLSmet $) /($ KmSmetCYP3A4 + CVLSmet $)$

;init AMLSmetCYP3A4 = 0 
;AMLSmetCYP2C19=Amount Smet metabolized in liver to S-EDDP by CYP2C19 ;AMLSmetCYP2C19' = (VmaxSmetCYP2C19*CVLSmet $) /($ KmSmetCYP2C19 + CVLSmet $)$ ;init AMLSMetCYP2C19=0

;metabolism described by enantiomeric interactions equations

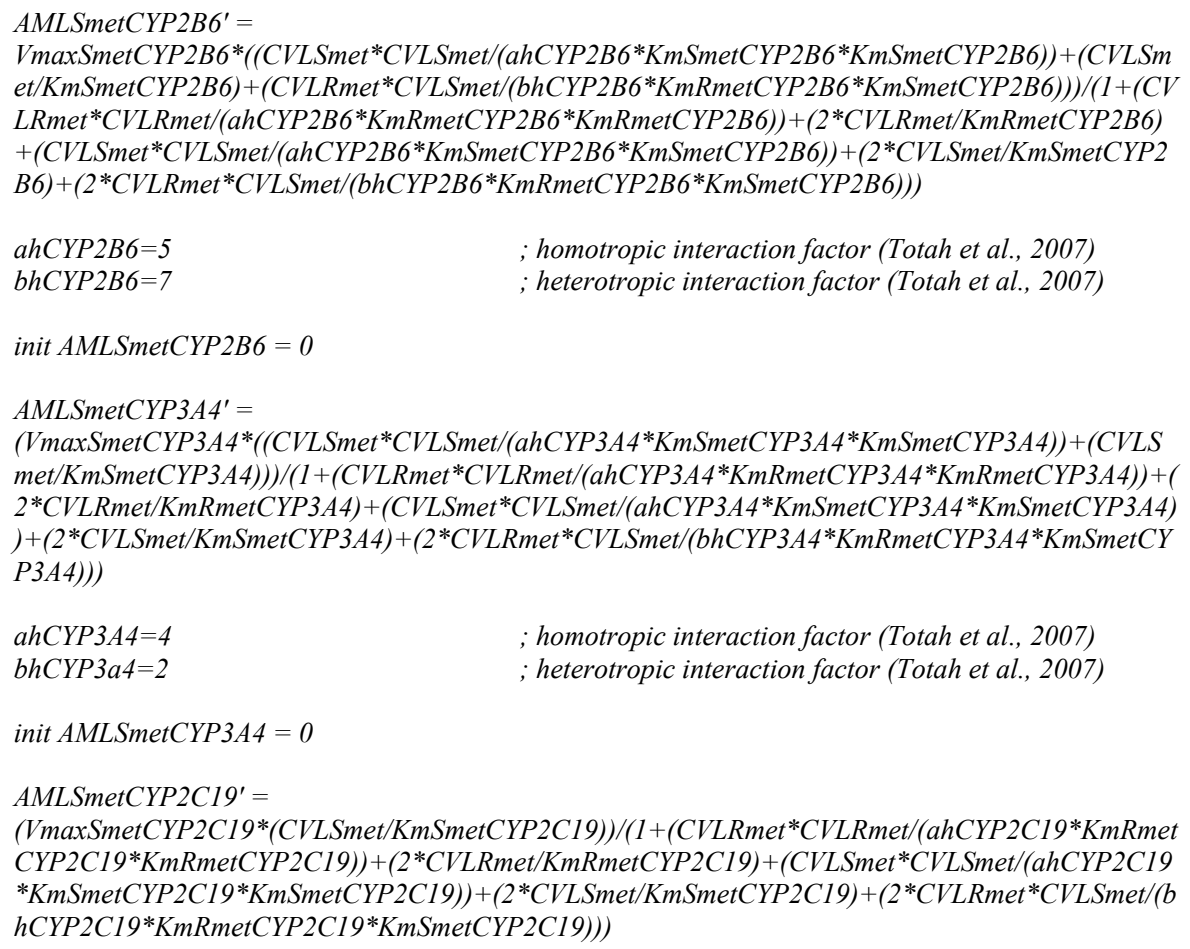

;kidney compartment

;AKRmet $=$ Amount R-methadone in kidney tissue $(\mu \mathrm{mol})$

AKRmet' $=\mathrm{QK} *($ CARmet - CVKRmet $)-$ ACLRmet'

Init AKRmet $=0$

CKRmet $=$ AKRmet $/ \mathrm{VK}$

CVKRmet $=$ CKRmet $/$ PKRmet

;ACLRmet=Amount R-methadone cleared renally

ACLRmet'=RCLRmet*CVKRmet

init ACLRmet $=0$

;AKSmet $=$ Amount S-methadone in kidney tissue $(\mu \mathrm{mol})$

AKSmet' $=\mathrm{QK} *($ CASmet - CVKSmet $)-$ ACLSmet'

Init $\mathrm{AKSmet}=0$

CKSmet $=$ AKSmet $/ \mathrm{VK}$

CVKSmet $=$ CKSmet $/$ PKSmet

;ACLSmet=Amount S-methadone cleared renally

ACLSmet' $=$ RCLSmet $*$ CVKSmet 
init ACLSmet $=0$

;Heart compartment

;AHRmet $=$ Amount R-methadone in heart tissue $(\mu \mathrm{mol})$

AHRmet' $=\mathrm{QH} *($ CARmet - CVHRmet $)$

Init AHRmet $=0$

CHRmet $=$ AHRmet $/ \mathrm{VH}$

CVHRmet $=$ CHRmet $/$ PHRmet

;AHSmet $=$ Amount S-methadone in heart tissue $(\mu \mathrm{mol})$

AHSmet' $=\mathrm{QH} *($ CASmet - CVHSmet $)$

Init $\mathrm{AHSmet}=0$

CHSmet $=$ AHSmet $/ \mathrm{VH}$

CVHSmet $=$ CHSmet $/$ PHSmet

; lung compartment

;ALuRmet $=$ Amount R-methadone in lung tissue $(\mu \mathrm{mol})$

ALuRmet' $=\mathrm{QC} *(\mathrm{CVRmet}-\mathrm{CALuRmet})$

Init ALuRmet $=0$

CLuRmet $=$ ALuRmet $/ \mathrm{VLu}$

CALuRmet $=$ CLuRmet $/$ PLuRmet

;ALuSmet $=$ Amount S-methadone in lung tissue $(\mu \mathrm{mol})$

ALuSmet' $=\mathrm{QC} *($ CVSmet - CALuSmet $)$

Init $\mathrm{ALuSmet}=0$

CLuSmet $=$ ALuSmet $/ \mathrm{VLu}$

CALuSmet $=$ CLuSmet $/$ PLuSmet

; arterial blood compartment

; CARmet $=$ Concentration arterial blood R-methadone

AARmet' $=\mathrm{QC} *($ CALuRmet- CARmet $)$;

Init AARmet $=0$

CARmet $=$ AARmet $/$ VA

; CASmet $=$ Concentration arterial blood S-methadone

AASmet' $=$ QC * (CALuSmet- CASmet);

Init AASmet $=0$

CASmet $=$ AASmet $/ \mathrm{VA}$

; venous blood compartment

;AVRmet $=$ amount venous blood R-methadone $(\mu \mathrm{mol})$

AVRmet' $=(\mathrm{QF} * \mathrm{CVFRmet}+\mathrm{QR} * \mathrm{CVRRmet}+\mathrm{QS} *$ CVSRmet $+\mathrm{QL} * \mathrm{CVLRmet}+\mathrm{QK} * \mathrm{CVKRmet}+$

$\mathrm{QH} * \mathrm{CVHRmet}-\mathrm{QC} * \mathrm{CVRmet})$

Init AVRmet $=0$

CVRmet $=($ AVRmet $/ \mathrm{VV})$

;AVSmet $=$ amount venous blood S-methadone $(\mu \mathrm{mol})$

AVSmet' $=(\mathrm{QF} *$ CVFSmet $+\mathrm{QR} * \mathrm{CVRSmet}+\mathrm{QS} *$ CVSSmet $+\mathrm{QL} * \mathrm{CVLSmet}+\mathrm{QK} * \mathrm{CVKSmet}+$

$\mathrm{QH} * \mathrm{CVHSmet}-\mathrm{QC} *$ CVSmet)

Init AVSmet $=0$

CVSmet $=($ AVSmet $/ \mathrm{VV})$

;Mass balance calculations

\{Mass Balance\}

TotalRMet' $=$ pulse $($ DOSERmet $*$ Fa, 0 , dose_int $)$

init TotalRmet $=1 \mathrm{E}-50$

CalculatedRmet $=$ AFRMet + ASRmet + ARRmet + ALRmet + AVRmet + AARmet + AGIRmet +

AMLRmetCYP2B6 + AMLRmetCYP3A4 + AMLRmetCYP2C19+ALuRmet + AKRmet + AHRmet +

ACLRmet 
ERRORRmet $=(($ TotalRmet - CalculatedRmet $) /($ TotalRmet $+1 \mathrm{E}-30)) * 100$

MASSBALRmet $=$ TotalRmet - CalculatedRmet +1

TotalSMet' $=$ pulse $($ DOSESmet $*$ Fa, 0 , dose int $)$

init TotalSmet $=1 \mathrm{E}-50$

CalculatedSmet $=$ AFSMet + ASSmet + ARSmet + ALSmet + AVSmet + AASmet + AGISmet +

AMLSmetCYP2B6 + AMLSmetCYP3A4 + AMLSmetCYP2C19 + ALuSmet + AKSmet + AHSmet +

ACLSmet

ERRORSmet $=(($ TotalSmet - CalculatedSmet $) /($ TotalSmet $+1 \mathrm{E}-30)) * 100$

MASSBALSmet $=$ TotalSmet - CalculatedSmet +1

;Calculation with model

\begin{tabular}{|c|c|}
\hline $\begin{array}{l}\text { CVRmetB }=\text { CVRmet } * \text { MWRmet } \\
\text { AUCRmet' }=\text { CVRmetB } \\
\text { init AUCRmet }=0\end{array}$ & $\begin{array}{l}\text {; concentration of R-methadone in venous blood }(\mu \mathrm{g} / \mathrm{L}) \\
\text {; calculate AUC for R-methadone }\end{array}$ \\
\hline $\begin{array}{l}\text { CVSmetB }=\text { CVSmet } * \text { MWSmet } \\
\text { AUCSmet' }=\text { CVSmetB } \\
\text { init AUCSmet }=0\end{array}$ & $\begin{array}{l}\text {; concentration of S-methadone in venous blood }(\mu \mathrm{g} / \mathrm{L}) \\
\text {; calculate AUC for S-methadone }\end{array}$ \\
\hline $\begin{array}{l}\text { CVheartRmet }=\text { CVHRmet } * \text { MWRmet } \\
\text { CVheartSmet }=\text { CVHSmet } * \text { MWSmet }\end{array}$ & $\begin{array}{l}\text {; concentration of R-methadone in the heart venuos blood }(\mu \mathrm{g} / \mathrm{L}) \\
\text {; concentration of S-methadone in the heart venuos blood }(\mu \mathrm{g} / \mathrm{L})\end{array}$ \\
\hline
\end{tabular}

\section{Supplementary materials 3}

Model code of Monte Carlo simulation for Caucasian population

\section{;CYP2B6 EM}

$; \mathrm{aCYP} 2 \mathrm{~B} 6 \mathrm{c}=\operatorname{init}(\exp (\operatorname{normal}(2.38,0.955)))$

;aCYP2B6 = IF aCYP2B6c >0.61 AND aCYP2B6c < 189.01 THEN aCYP2B6c ELSE 100001 ;Values higher or lower than 3 times the SD are removed

\section{;CYP2B6 PM}

aCYP2B6c = init $(\exp (\operatorname{normal}(0.99,1.269)))$

aCYP2B6 = IF aCYP2B6c >0.06 AND aCYP2B6c < 120.66 THEN aCYP2B6c ELSE 100001 ;Values higher or lower than 3 times the SD are removed

;CYP3A4 general population $\mathrm{n}=713$

aCYP3A4c = init $(\exp ($ normal $(4.28,0.71)))$

aCYP3A4 = IF aCYP3A4c $>8.58$ AND aCYP3A4c $<608.6$ THEN aCYP3A4c ELSE $100001 ;$ Values higher or lower than 3 times the $\mathrm{SD}$ are removed

;CYP2C19 general population $\mathrm{n}=76$

aCYP2C19c $=$ init $(\exp (\operatorname{normal}(2.14,0.717)))$

aCYP2C19 = IF aCYP2C19c >0.99 AND aCYP2C19c $<73.12$ THEN aCYP2C19c ELSE 100001 ;Values

higher or lower than 3 times the $\mathrm{SD}$ are removed 


$$
5
$$




\section{Chapter 5}

\section{A new approach methodology for the prediction of (nor)ibogaine- induced cardiotoxicity in humans}

Miaoying Shi, Sebastiaan Wesseling, Hans Bouwmeester, Ivonne M. C. M. Rietjens

Submitted 


\begin{abstract}
The development of non-animal based New Approach Methodologies (NAMs) for chemical risk assessment and safety evaluation is urgently needed. The aim of the present study was to investigate the applicability of an in vitro in silico approach to predict human cardiotoxicity of the herbal alkaloid ibogaine and its metabolite noribogaine, being promising anti-addiction drugs. Physiologically based kinetic (PBK) models were developed using in silico-derived parameters and biokinetic data obtained from in vitro liver microsomal incubations and Caco2 transport studies. Human induced pluripotent stem cell-derived cardiomyocytes combined with the multi-electrode array (MEA) assay were used to determine in vitro concentrationdependent cardiotoxicity reflected by prolongation of field potential duration, which was subsequently translated to in vivo dose-dependent QTc prolongation using PBK model based reverse dosimetry. Results showed that the predictions matched well with available in vivo kinetic data and QTc data for ibogaine and noribogaine available in literature, indicating a good performance of the NAM. Benchmark dose analysis of the predicted dose response curves adequately predicted the onset of in vivo cardiotoxicity detected by QTc prolongation upon oral exposure to ibogaine and noribogaine. The present study provides an additional proof of principle of using PBK modeling-based reverse dosimetry as a NAM to predict human cardiotoxicity.
\end{abstract}




\section{Introduction}

In line with the 3 Rs principle, the development of non-animal based novel methods has been a leading research topic towards New Approach Methodologies (NAMs) for chemical risk assessment and safety evaluation (Andersen et al., 2019; ICCVAM, 2018; Taboureau et al., 2020). The NAMs using in vitro and in silico models have become increasingly important for predicting human toxicity as they are high throughput in generating data and reduce animal use and costs (Bos et al., 2020; Patterson et al., 2020). Within the frame of NAMs, the biological effects of chemicals are characterized by in vitro toxicity assays with target organ specificity and reflecting the relevant mode of action, while the biokinetics related to absorption, distribution, metabolism and distribution (ADME) can be captured by using in vitro and/or computational models (Andersen et al., 2019; Punt et al., 2020). One good example of such NAMs is so-called physiologically based kinetic (PBK) modelling-based reverse dosimetry, which has been shown to be of use for quantitative in vitro in vivo extrapolation (QIVIVE) enabling definition of in vivo dose response curves for different toxic endpoints (Abdullah et al., 2016; Gilbert-Sandoval et al., 2020; Louisse et al., 2010; Rietjens et al., 2011; Strikwold et al., 2017; Zhao et al., 2019), including cardiotoxicity (Shi et al., 2020a).

In our previous work, we demonstrated that in vivo methadone-induced QTc prolongation (heart rate corrected time duration from ventricular depolarization to repolarization) can be adequately predicted based on in vitro cardiotoxic effects of methadone on human induced pluripotent stem cell-derived cardiomyocytes (hiPSC-CMs) combined with PBK modelling (Shi et al., 2020a). Given that cardiotoxicity is one of the most common toxic endpoints and a main concern for discontinuing drug development (Ovics et al., 2020; Pang et al., 2019), it is of importance to validate the applicability and accuracy of this newly developed NAM by providing additional proofs of principle for the evaluation of cardiotoxicity. Thus the aim of the present study was to apply the developed PBK modelling-based reverse dosimetry approach to predict the cardiotoxicity of ibogaine and its metabolites, which are plant-based substances that attracted special attention due to their potential cardiotoxicity occurring in the clinical setting (Schep et al., 2016).

Ibogaine is an indole alkaloid naturally occurring in the West African shrub Tabernanthe $i b o g a$, which was traditionally used for medical treatment and religious ceremonies (Davis et al., 2017; Goutarel et al., 1993; Litjens and Brunt, 2016; Mash et al., 2018). Nowadays ibogaine is banned or allowed only under medical supervision in most countries due to its psychoactive 
properties with an exception of New Zealand Noller et al., 2018). It is used given that a single high dose of ibogaine can be effective for reducing drug-induced withdrawal symptoms in human (Alper, 1999; Mash et al., 2018; Noller et al., 2018). Ibogaine is mainly metabolized by the hepatic cytochromes P450 (CYPs) via O-demethylation to the primary metabolite noribogaine, which is also psychoactive and has pharmacological effects (Obach et al., 1998; Glue et al., 2016; Mash et al., 2016; Litjens and Brunt, 2016). The major enzyme involved in the conversion of ibogaine to noribogaine has been identified in both in vitro and in vivo studies to be CYP2D6 (Obach et al., 1998; Glue et al., 2015b), with minor contributions from CYP2C9 and CYP3A4 (Obach et al., 1998). Subsequently, noribogaine is cleared via glucuronidation to noribogaine glucuronide (Glue et al., 2016; Glue et al., 2015a; Glue et al., 2015b) (Figure 1).

The efficacy and safety of ibogaine and noribogaine for the treatment of drug addiction has been under debate over decades. Despite evidence obtained in preclinical and clinical studies showing promising pharmacological efficacy, cardiotoxicity was identified as the major safety concern for its clinical use (Schep et al., 2016). Several fatalities and case reports described patients who received a high dose of ibogaine after which they experienced prolonged QTc interval, which could further develop to cardiac arrythmia and even sudden death (Asua, 2013; Grogan et al., 2019; Hildyard et al., 2016; Hoelen et al., 2009; O'Connell et al., 2015; Paling et al., 2012; Pleskovic et al., 2012; Steinberg and Deyell, 2018; Vlaanderen et al., 2014). Furthermore, Glue et al. (2016) observed a dose-dependent effect of noribogaine on QTc prolongation in opioid-dependent patients. The observed QT prolongation could be associated with the potential inhibitory effect of both compounds on human ether-à-go-go-related gene (hERG) channels that play a critical role in cardiac repolarization (Martin et al., 2004). Based on in vitro studies using the electrophysiological-based patch clamp technique, both ibogaine and noribogaine were reported to block the hERG channels with similar potency (Alper et al., 2016; Koenig et al., 2014; Rubi et al., 2017).

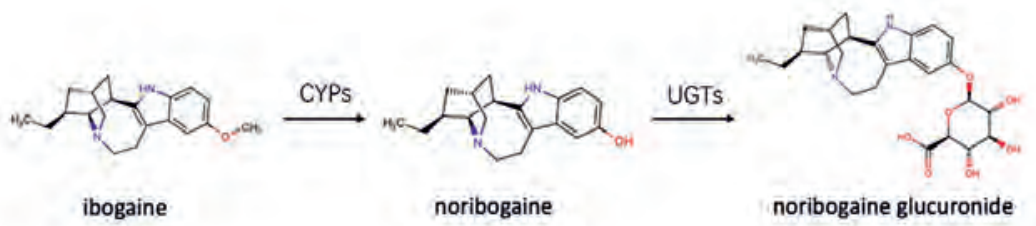

Figure 1 Metabolic pathway of ibogaine to noribogaine by cytochromes P450 (CYPs) and subsequent conversion of noribogaine to noribogaine glucuronide by glucuronosyltransferases (UGTs). 
In the present study, to predict the cardiotoxicity upon oral exposure to ibogaine or noribogaine using PBK model based reverse dosimetry, the in vitro cardiotoxicity of the two compounds was quantified in hiPSC-CMs using the multiple-electrode array (MEA) technique. The obtained data were subsequently combined with in vitro biokinetic data and used in a PBK model based reverse dosimetry approach to predict human dose-response curves. The hiPSCCM MEA assay quantifies the extracellular field potential duration corrected for beat rate (FPDc) as an in vitro surrogate of QTc prolongation in the human electrocardiogram (ECG). The biokinetic data describing absorption and metabolism of ibogaine and noribogaine, obtained from in vitro experiments, were integrated in a PBK model for ibogaine with a submodel for noribogaine in human. Subsequently the PBK model was used to translate the in vitro concentration-dependent cardiotoxicity to the predicted dose-dependent cardiotoxic effects on QTc prolongation in human. The obtained data were compared to reported human in vivo data on ibogaine and noribogaine-induced QTc prolongation. Benchmark dose (BMD) analysis of the predicted in vivo dose-response curves was performed to allow comparison to available data on human cardiotoxicity and validate the predictions, thus providing insight in the use of the NAM to evaluate the risk of ibogaine and noribogaine-induced cardiotoxicity in the clinical setting.

\section{Materials and methods}

\subsection{Chemicals and biological materials}

Ibogaine hydrochloride (99.5\%), noribogaine hydrochloride (98.8\%) and noribogaine glucuronide lithium salt (97.4\%) were purchased from TLC Pharmaceutical Standards Ltd. (Newmarket, Ontario, Canada,). Antipyrine ( $\geq 99 \%$ ), bovine serum albumin (BSA, $\geq 96 \%$ ), dofetilide $(\geq 98 \%)$, fibronectin, fluorescein $(95 \%)$, isoproterenol hydrochloride $(\geq 98 \%)$, methadone hydrochloride ( $\geq 98 \%$ ), methanesulphonic acid ( $\geq 99 \%$ ) and Tris (hydroxymethyl) aminomethane (Tris), were purchased from Sigma-Aldrich (Zwijndrecht, The Netherlands). Methadone was used under the opium exemption license number $10478303 \mathrm{WCO}$, registered at Farmatec (executive organization of the Ministry of Health, Welfare and Sport, The Hague, The Netherlands). Dimethyl sulfoxide (DMSO, 99.7\%) and sodium hydrogen carbonate $\left(\mathrm{NaHCO}_{3}, \geq 99 \%\right)$ were obtained from Merck (Schiphol-Rijk, The Netherlands). Acetonitrile (UPLC/MS grade) was obtained from Biosolve BV (Valkenswaard, The Netherlands). Formic acid (FA) was purchased from VWR International (Amsterdam, The Netherlands). 
hiPSC-CM (Pluricyte ${ }^{\circledR}$ Cardiomyocytes, cat\# PCMI-1031-1) and Pluricyte ${ }^{\circledR}$ Cardiomyocyte medium were purchased from Ncardia (Leiden, The Netherlands). The human colon carcinoma cell line Caco-2 was obtained from ATCC (Manassas, VA, USA). Dulbecco's modified Eagle's medium (DMEM, GlutaMAXTM containing 4.5 g/L D-glucose and pyruvate), Hank's balanced salt solution (HBSS) without phenol red and phosphate-buffered saline (PBS) were obtained from Gibco (Paisley, Scotland, UK). Non-essential amino acids (NEAA) and penicillin-streptomycin (P/S) were purchased from Gibco (Grand Island, New York, USA). Fetal Bovine Serum (FBS) was purchased from Bodinco BV (Alkmaar, The Netherlands). Pooled human liver microsomes (Corning ${ }^{\circledR}$ UltraPool $^{\text {TM }}$ HLM 150, pooled from 150 donors with mixed gender), reduced nicotinamide adenine dinucleotide phosphate (NADPH) regenerating system solution $A$ and solution $B$, glucuronosyltransferase (UGT) reaction mix system solution A and solution B were purchased from Corning (Woburn, MA, USA). Pooled human plasma and rapid equilibrium dialysis (RED) materials, including RED inserts, RED base plates and sealing tape were obtained from Thermo Fisher Scientific (Bleiswijk, The Netherlands).

\subsection{In vitro cardiotoxicity of ibogaine and noribogaine in the hiPSC-CM}

\section{MEA assay}

\subsection{1. hiPSC-CM culture}

Pluricyte ${ }^{\circledR}$ Cardiomyocytes (Ncardia) were prepared according to the manufacturer's protocol as previously described in Shi et al. (2020a). Prior to seeding, precoating was performed by adding $2 \mu 1$ of $50 \mu \mathrm{g} / \mathrm{ml}$ fibronectin in each well of the MEA chips (60-6well MEA200/30iRTi-tcr, MCS GmbH, Ruetlingen, Germany) and the coating solution was removed after 3-h incubation at $37{ }^{\circ} \mathrm{C}$ with $5 \% \mathrm{CO}_{2}$. Cardiomyocytes were taken from liquid nitrogen and immediately thawed in the incubator at $37^{\circ} \mathrm{C}$ for exactly 4 min and then mildly transferred to a $50 \mathrm{ml}$ tube. $1 \mathrm{ml}$ serum free Pluricyte ${ }^{\circledR}$ Cardiomyocyte Medium (Ncardia) was added to the original vial for rinsing and drop-wisely transferred to the $50 \mathrm{ml}$ tube, followed by a drop-wise addition of $5 \mathrm{ml}$ medium. Manually cell counting was performed using Buerker-Tuerk Counting Chamber (Marienfeld Superior GmbH \& Co. KG, Lauda-Königshofen, Germany) followed by a centrifugation at $300 \mathrm{~g}$ for $3 \mathrm{~min}$ at $25{ }^{\circ} \mathrm{C}$. After removing the supernatant, medium was carefully added to reach the aimed concentration being $10^{4}$ cells $/ \mu 1$. Subsequently, $2 \mu 1$ of cell suspension was added to each well of the MEA chips which were incubated at $37^{\circ} \mathrm{C}$ with $5 \% \mathrm{CO}_{2}$ for $3 \mathrm{~h}$. Then $200 \mu \mathrm{l}$ of medium was filled into each well and cardiomyocytes were maintained for 7 days $\left(37^{\circ} \mathrm{C}\right.$ with $\left.5 \% \mathrm{CO}_{2}\right)$ with medium refreshing every 2 days. 


\subsubsection{MEA recording}

The exposure and the recording of spontaneous beating of hiPSC-CMs were conducted as previously described (Shi et al., 2020a). Stock solutions of ibogaine were prepared in acetonitrile/water $(50 / 50 \mathrm{v} / \mathrm{v})$. Noribogaine and two references, dofetilide and isoproterenol, were dissolved in DMSO. All stock solutions were diluted in culture medium to make exposure medium with the final concentration of $0.05 \%(\mathrm{v} / \mathrm{v})$ acetonitrile or $0.1 \%(\mathrm{v} / \mathrm{v})$ DMSO. Based on cytotoxicity and relevant human plasma concentrations observed upon oral administration of ibogaine and noribogaine, the following concentrations were tested, 0 (control), 0.03, 0.1, $0.2,0.3,0.4,0.5,1 \mu \mathrm{M}$ (ibogaine) and 0 (control), 0.03, 0.1, 0.2, 0.3, 0.4, 1, $3 \mu \mathrm{M}$ (noribogaine). The test concentrations were 0 (control), $0.1,0.3,1,3,10 \mathrm{nM}$ for dofetilide and 0 (control), 3 , $10,30,100,300 \mathrm{nM}$ for isoproterenol, which are typical responsive concentrations in the hiPSC-CM MEA assay (Zwartsen et al. 2019).

At day 7-9 post-seeding, extracellular field potentials of cardiomyocytes (Figure 2) were accessed by using the MEA2100-system (MCS GmbH) equipped with a chamber and a heating controller that guaranteed a stable atmosphere $\left(37{ }^{\circ} \mathrm{C}\right.$ with $\left.5 \% \mathrm{CO}_{2}\right)$. A 20 min equilibration period was applied prior to the compound/ vehicle exposure. Measurements started with replacing half of the medium in each well by fresh medium containing $0.1 \%(\mathrm{v} / \mathrm{v})$ acetonitrile or $0.2 \%(\mathrm{v} / \mathrm{v})$ DMSO to reach a final concentration of $0.05 \%(\mathrm{v} / \mathrm{v})$ acetonitrile or $0.1 \%(\mathrm{v} / \mathrm{v})$ DMSO, which was defined as baseline condition. Following the same way, each concentration of the model compounds was cumulatively added to the well increasing the concentration at each subsequent addition (Ando et al., 2017; Nozaki et al., 2017). In each MEA chip, five of the six wells were exposed to model or reference compounds while one well served as the vehicle control analyzed during a similar period of time as the other wells to enable corrections for time-, addition- and vehicle-dependent effects on the field potentials. At each concentration, exposure was conducted for $15 \mathrm{~min}$ followed by a 2 min recording of extracellular field potentials. Data were recorded using Cardio 2D software Version 2.12.0 (MCS GmbH) with a sample frequency of $10 \mathrm{kHZ}$ and a $0.1-3.5 \mathrm{kHz}$ band-pass filter.

\subsubsection{Data analysis and statistics}

Raw data obtained from the hiPSC-CM MEA assay were analyzed using Multiwell Analyzer software Version 1.8.6.0 (MCS GmbH). Electrodes with field potentials of good quality, being the ones with amplitudes of depolarization and repolarization peaks higher than $200 \mu \mathrm{V}$ and $20 \mu \mathrm{V}$, respectively, were selected for further analysis (Ando et al., 2017; Sala et al., 2017; Shi 
et al., 2020a). Subsequently, two parameters reflecting electrophysiological activity of cardiomyocytes, namely field potential duration (FPD, duration between the beginning of the sodium spike and the repolarizing peak) and RR interval (duration between two depolarization peaks), were measured from the $2 \mathrm{~min}$ recording for each concentration.

In line with other MEA studies, the Fridericia formula (Eq. 1) was applied to correct for the effect of beat rate on FPD (Ando et al., 2017; Kitaguchi et al., 2017; Vandenberk et al., 2016):

$$
\mathrm{FPDc}=\frac{\mathrm{FPD}}{\sqrt[3]{\mathrm{RR} \text { interval }}}
$$

Where the FPD and RR-interval are expressed in seconds. Well-based FPDc were determined by calculating the relative percentage of FPDc for the exposure measurements compared to FPDc at baseline conditions $(0.05 \%(\mathrm{v} / \mathrm{v})$ acetonitrile or $0.1 \%(\mathrm{v} / \mathrm{v}) \mathrm{DMSO})$ set at $100 \%$. The potential effects of time, addition and vehicle on well-based FPDc were corrected for by subtracting the responses obtained from the corresponding time-matched vehicle control well. Irregular waveforms (Figure S1) on field potential including arrhythmia-type changes, a flattened unclear second peak and beating arrest may occur at high concentrations (Kitaguchi et al., 2017; Shi et al., 2020a; Zwartsen et al., 2019). For deriving concentration-response curves of model compounds, concentrations inducing irregular waveforms were excluded given the FPD and RR interval could not be defined.

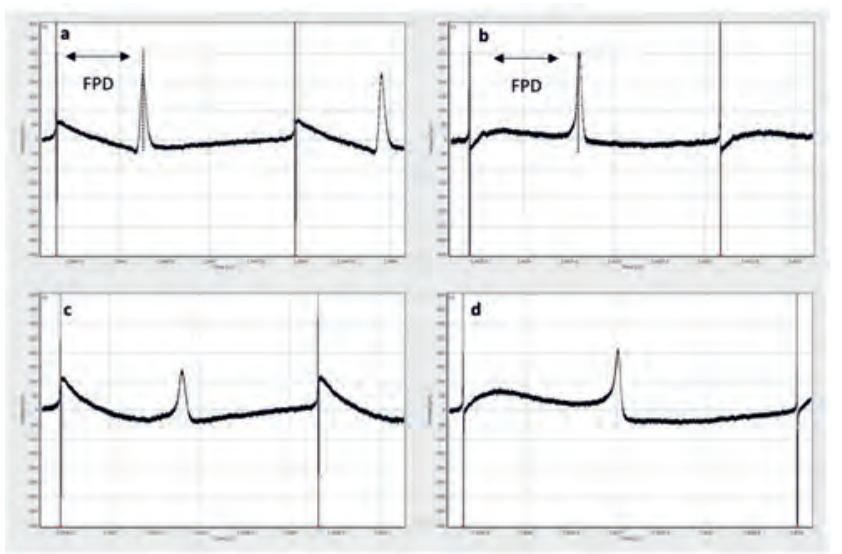

Figure 2 Normal and prolonged waveforms of field potential observed in human induced pluripotent stem cell-derived cardiomyocytes using the multiple-electrode array. a, field potential duration (FPD) at baseline condition with the treatment of $0.05 \%(\mathrm{v} / \mathrm{v})$ acetonitrile. b, FPD at baseline condition with the treatment of $0.1 \%(\mathrm{v} / \mathrm{v})$ DMSO. c, prolonged FPD observed at $0.4 \mu \mathrm{M}$ ibogaine. $\mathrm{d}$, prolonged FPD observed at $0.4 \mu \mathrm{M}$ noribogaine. 
To compare the potency of model compounds, BMD analysis was used to derive effective concentrations causing a defined \% increase in the FPDc compared to the baseline control as described in the "Benchmark dose analysis" section. The concentration-response curves were plotted using GraphPad Prism 5.0 with four-parameters logistic fit (GraphPad Software Inc., San Diego, USA). Data were collected from three independent experiments with six well replicates (two replicates for dofetilide and isoproterenol) in each experiment. Each data point is presented as the mean value \pm standard deviation (SD) of the three independent replicates. One-way analysis of variance (ANOVA) followed by Dunnett test were conducted to assess the effect of ibogaine and noribogaine on FPDc using GraphPad Prism 5.0 (GraphPad Software Inc.). Significance was determined at $p<0.05$.

\subsection{In vitro experimental biokinetic parameters for the PBK models}

\subsubsection{In vitro intestinal transport studies}

To estimate the intestinal absorption of ibogaine and noribogaine, a Caco- 2 transport study was performed to determine the apparent permeability coefficients $\left(\mathrm{P}_{\mathrm{app}}\right)$ using a previously developed method with minor modifications (Hubatsch et al., 2007; Strikwold et al., 2017). Caco-2 cells (passages 10-15 post -thawing) were seeded onto the insert of Costar 12-well transwell plates (Corning, $0.4 \mu \mathrm{m}$ pored polycarbonate membrane, $12 \mathrm{~mm}$ diameter). The apical compartment of each well was filled with $0.5 \mathrm{ml}$ of a $4 \times 10^{4}$ cell suspension in culture medium consisting of DMEM supplemented with $10 \%$ (v/v) FBS, 1\% (v/v) P/S and 1\% (v/v) NEAA and the basolateral compartment was filled with $1.5 \mathrm{ml}$ culture medium. The plate was incubated under atmospheric conditions at $37{ }^{\circ} \mathrm{C}$ and $5 \% \mathrm{CO}_{2}$ for $21-23$ days, which allows cells to grow and differentiate into a confluent monolayer. Cell culture medium was changed every 2 days.

Stock solutions of ibogaine were prepared in acetonitrile/water $(50 / 50 \mathrm{v} / \mathrm{v})$. Noribogaine, methadone (reference compound), antipyrine (passive transcellular control for the transport studies) and fluorescein (passive paracellular control for the transport studies) were dissolved in DMSO. Stock solutions were diluted 200-fold resulting a final test concentration of $50 \mu \mathrm{M}$ (125 nM for fluorescein) at which cytotoxicity was not observed (data not shown). The final concentration of acetonitrile or DMSO was $0.25 \%(\mathrm{v} / \mathrm{v})$ or $0.5 \%(\mathrm{v} / \mathrm{v})$, respectively.

For the transport experiment, HBSS containing $10 \mathrm{mM}$ methanesulfonic acid, $\mathrm{NaHCO}_{3}$ (final concentration $0.35 \mathrm{mg} / \mathrm{ml}$ ) and the respective test compounds was used as exposure medium ( $\mathrm{pH}$ 6.5) in the apical compartment. HBSS containing $30 \mathrm{mg} / \mathrm{ml} \mathrm{BSA}$ was used as 
medium in the basolateral compartment ( $\mathrm{pH} 7.4$ ). $1.5 \mathrm{ml}$ basolateral transport medium was first added, and the experiment was initiated by adding $0.5 \mathrm{ml}$ exposure medium to the apical compartment. The plate was incubated at $37{ }^{\circ} \mathrm{C}$ with $5 \% \mathrm{CO}_{2}$ for $20 \mathrm{~min}$. Subsequently, an aliquot of $75 \mu \mathrm{l}$ sample was collected from the basolateral compartment and transferred to a tube containing $150 \mu 1$ ice-cold acetonitrile. To measure the mass balance (recovery), $75 \mu 1$ exposure buffer was also collected from the apical compartment before and after the incubation and added to the tubes containing $150 \mu \mathrm{l}$ ice-cold acetonitrile. The insert filters were washed with PBS then cut out and transferred to $200 \mu \mathrm{l}$ ice-cold acetonitrile for a 15 min sonification. Samples were left on ice for $20 \mathrm{~min}$ and centrifuged for $45 \mathrm{~min}$ at $18,000 \mathrm{~g}$, after which supernatants were collected for LC-MS/MS analysis.

To check the linearity of transport of test compounds, $75 \mu 1$ sample was collected from the basolateral compartment at 1, 5, 10, 20, 30 and $60 \mathrm{~min}$ of incubation and added to $150 \mu \mathrm{l}$ icecold acetonitrile. After each collection, an equal volume of basolateral transport medium was added back as compensation. The calculation for each time point was corrected for the dilutions.

The integrity of the Caco-2 cell monolayer was checked by measuring the transepithelial electrical resistance (TEER) using a Millicell ERS-2 Volt-Ohm Meter (EMD Millipore Corporation, California, USA). Prior to the transport experiment, cells with a TEER value between 600 and $800 \Omega \mathrm{cm}^{2}$ were chosen for the transport study. After the experiment, cells with a change of TEER value larger than $20 \%$ were excluded from further analysis of the $\mathrm{P}_{\text {app }}$ value (Bentz et al., 2013). Additionally, the integrity was also checked by measuring the transport of fluorescein, and cells with a transport of fluorescein less than $1 \%$ were considered suitable for the analysis.

The $\mathrm{P}_{\text {app }}(\mathrm{cm} / \mathrm{s})$ value was calculated using Eq. 2 as follows (Hubatsch et al., 2007; Strikwold et al., 2017):

$$
\mathrm{P}_{\mathrm{app}}=\frac{\Delta \mathrm{Q} / \Delta \mathrm{t}}{\mathrm{A} \times \mathrm{C}_{0}}
$$

where $\Delta \mathrm{Q} / \Delta \mathrm{t}(\mathrm{nmol} / \mathrm{s})$ is the amount of the test compound transported to the basolateral compartment over the incubation time, $\mathrm{A}\left(\mathrm{cm}^{2}\right)$ is the surface area of the filter and $\mathrm{C}_{0}(\mu \mathrm{M})$ is the initial concentration of the test compound in the apical compartment. The mass balance was calculated by comparing the total amount of compound in the apical compartment, basolateral compartment and filter to the initial amount. The results were obtained from three independent experiments including four replicates for ibogaine and noribogaine and two 
replicates for methadone and antipyrine in each independent experiment. Data are presented as the mean value $\pm \mathrm{SD}$.

Using methadone as a reference compound, the in vivo oral absorption rate constant (ka) for ibogaine and noribogaine were calculated by multiplying the corresponding ratio of $\mathrm{P}_{\text {app }}$ to $\mathrm{P}_{\mathrm{app}}$ of methadone by a mean ka value of methadone being $0.59 / \mathrm{h}$ reported in human studies (Foster et al., 2000; Wolff et al., 2000).

\subsubsection{In vitro microsomal incubations}

To determine kinetic parameters for the conversion of ibogaine to noribogaine, in vitro microsomal incubations were performed as described before (Shi et al., 2020a) with minor modifications. Incubation mixtures contained the NADPH regeneration system (final concentrations $1.3 \mathrm{mM} \mathrm{NADP}^{+}, 3.3 \mathrm{mM}$ glucose-6-phosphate, $0.4 \mathrm{U} / \mathrm{ml}$ glucose-6-phosphate dehydrogenase and $3.3 \mathrm{mM} \mathrm{MgCl} 2$ ) and ibogaine at final concentrations from 0 to $25 \mu \mathrm{M}$, in $100 \mathrm{mM}$ Tris-HCl buffer ( $\mathrm{pH}$ 7.4) with a total volume of $160 \mu$ l. Stock solutions of ibogaine were prepared in acetonitrile/water $(50 / 50 \mathrm{v} / \mathrm{v})$, and were diluted 50 times to reach the test concentrations in the incubation mixtures. Samples were pre-incubated at $37^{\circ} \mathrm{C}$ for $1 \mathrm{~min}$ and a final concentration of $0.25 \mathrm{mg} / \mathrm{ml}$ liver microsomal protein was added to initiate the reaction. After 2.5 min incubation in a shaking water bath at $37{ }^{\circ} \mathrm{C}$, the reaction was terminated by adding $40 \mu \mathrm{l}$ ice-cold acetonitrile. Control incubations were performed in the absence of NADPH which was replaced with Tris-HCl.

The formation of the glucuronide of noribogaine was investigated in a similar manner as described above. A total volume of $200 \mu \mathrm{l}$ sample contained (final concentrations) UGT reaction system (5 $\mathrm{mM}$ uridine 5'-diphosphoglucuronic acid (UDPGA), $8 \mathrm{mM} \mathrm{MgCl}_{2}, 25$ $\mu \mathrm{g} / \mathrm{ml}$ alamethicin) and $0.5 \mathrm{mg} / \mathrm{ml}$ liver microsomal protein in $100 \mathrm{mM}$ Tris- $\mathrm{HCl}$ (pH 7.4). Samples were placed on ice for $30 \mathrm{~min}$ which allows the pore forming peptide alamethicin to boost the glucuronidation activity (Fisher et al., 2000; Ning et al., 2017). After pre-incubating samples at $37^{\circ} \mathrm{C}$ for $5 \mathrm{~min}$, reactions were started by adding noribogaine from 100 times concentrated stock solutions in DMSO at final concentrations ranging from 0-750 $\mu \mathrm{M}$. The incubation time was $40 \mathrm{~min}$ and reactions were terminated by adding $50 \mu \mathrm{l}$ ice-cold acetonitrile. Control incubations were performed in the absence of UDPGA which was replaced with Tris$\mathrm{HCl}$.

Test concentrations of ibogaine and noribogaine were chosen at relevant human plasma concentrations and enabled the determination of Michaelis-Menten kinetic parameters. 
Optimizations of experiments demonstrated that the reaction rates for the formation of noribogaine were linear with time up to $20 \mathrm{~min}$ and with protein concentration of up to $1 \mathrm{mg} / \mathrm{ml}$. For the formation of noribogaine glucuronides, reaction rates were shown to be linear with time up to $120 \mathrm{~min}$ and with protein concentration up to $0.75 \mathrm{mg} / \mathrm{ml}$. After termination, samples were centrifuged at $18,000 \mathrm{~g}$ for $5 \mathrm{~min}$ at $4{ }^{\circ} \mathrm{C}$. The supernatant was collected for the quantification of ibogaine, noribogaine and noribogaine glucuronide by liquid chromatography-mass spectrometry (LC-MS/MS) as described in the "LC-MS/MS analysis" section.

For the kinetic analysis, the data for the formation of noribogaine and noribogaine glucuronide were fitted to the Michaelis-Menten Eq. 3 using GraphPad Prism 5.0 (GraphPad Software Inc.):

$$
\mathrm{V}=\frac{\mathrm{V}_{\max } \mathrm{x}[\mathrm{S}]}{\mathrm{K}_{\mathrm{m}}+[\mathrm{S}]}
$$

where $[\mathrm{S}]$ is the substrate concentration $(\mu \mathrm{M})$ and $\mathrm{v}$ is the rate of metabolite formation ( $\mathrm{nmol} / \mathrm{min} / \mathrm{mg}$ protein). $\mathrm{V}_{\max }$ is the apparent maximum velocity ( $\mathrm{nmol} / \mathrm{min} / \mathrm{mg}$ protein) and $\mathrm{K}_{\mathrm{m}}$ the apparent Michaelis-Menten constant $(\mu \mathrm{M})$. The in vitro catalytic efficiency expressed in $\mu 1 / \mathrm{min} / \mathrm{mg}$ microsomal protein was calculated by dividing $\mathrm{V}_{\max }$ by $\mathrm{K}_{\mathrm{m}}$. Data were collected from three independent experiments and each data point is presented as the mean value $\pm \mathrm{SD}$.

\subsection{Determination of plasma protein and in vitro medium binding}

The rapid equilibrium dialysis (RED) assay was conducted to determine the unbound fraction $\left(f_{u}\right)$ of ibogaine and noribogaine in pooled human plasma and in the in vitro medium used in the hiPSC-CM MEA assay as previously described (Shi et al., 2020a). The stock solutions of ibogaine prepared in acetonitrile/water $(50 / 50 \mathrm{v} / \mathrm{v})$ and noribogaine dissolved in DMSO were diluted 100-fold in human plasma or in vitro medium to reach the final concentration of $10 \mu \mathrm{M}$ in test sample solutions. A $300 \mu \mathrm{l}$ aliquot of test sample solution was added to the sample chamber and $500 \mu \mathrm{l}$ PBS was added to the buffer chamber of the RED insert, after which dialysis was performed on an orbital shaker at $37^{\circ} \mathrm{C}$ at $250 \mathrm{rpm}$ for $5 \mathrm{~h}$. Following dialysis, an aliquot of $25 \mu \mathrm{l}$ was collected from both the sample and buffer chamber and diluted with $25 \mu 1$ of PBS (to the aliquot from the sample chamber) and plasma or in vitro medium (to the aliquot from the buffer chamber) to eliminate potential matrix effects. Subsequently, post-treatment samples were precipitated using $300 \mu \mathrm{l}$ cold acetonitrile/water $(50 / 50 \mathrm{v} / \mathrm{v})$ and left on ice for $30 \mathrm{~min}$ followed by a centrifugation for $45 \mathrm{~min}$ at $18,000 \mathrm{~g}$. Supernatants were collected for 
LC-MS/MS analysis. The fraction unbound $\left(f_{u}\right)$ was calculated with Eq. 4 (van Liempd et al., 2011; Waters et al., 2008):

$$
\mathrm{f}_{\mathrm{u}}=\frac{\text { concentration in buffer chamer }}{\text { concentration in sample chamer }}
$$

The measurements were performed in triplicate in three independent experiments. Results are presented as the mean value \pm SD.

\subsection{LC-MS/MS analysis}

The identification and quantification of compounds in samples from the Caco-2 transport studies, microsomal incubation and RED assay were performed by LC-MS/MS analysis using a Shimadzu Nexera XR LC-40D SR UPLC system coupled with a Shimadzu LCMS-8045 mass spectrometer (Kyoto, Japan). The compounds were separated by a Phenomenex Kinetex ${ }^{\circledR}$ C18 column $(2.1$ x $50 \mathrm{~mm} 1.7 \mu \mathrm{m}, 100 \AA)$ connected to a precolumn and detected by a Shimadzu LCMS-8045 triple quadrupole with electrospray ionization (ESI) interface. The instrument was operated in positive mode and multiple reaction monitoring (MRM, $\mathrm{N}_{2}$ collision gas) mode. The MRMs of m/z $311.15\left(\mathrm{MH}^{+}\right)$to $122.2(\mathrm{CE}:-33 \mathrm{kV}), \mathrm{m} / \mathrm{z} 311.15\left(\mathrm{MH}^{+}\right)$to $174.2(\mathrm{CE}:-$ $36 \mathrm{kV})$ and $\mathrm{m} / \mathrm{z} 311.15\left(\mathrm{MH}^{+}\right)$to $124.15(\mathrm{CE}:-31 \mathrm{kV})$ were used to analyse ibogaine. The MRMs of m/z $297\left(\mathrm{MH}^{+}\right)$to $122.15(\mathrm{CE}:-33 \mathrm{kV}), \mathrm{m} / \mathrm{z} 297\left(\mathrm{MH}^{+}\right)$to $160.2(\mathrm{CE}:-35 \mathrm{kV})$ and $\mathrm{m} / \mathrm{z} 297\left(\mathrm{MH}^{+}\right)$to $146.25(\mathrm{CE}:-45 \mathrm{kV})$ were used to analyse ibogaine. The MRMs of $\mathrm{m} / \mathrm{z}$ $473.15\left(\mathrm{MH}^{+}\right)$to $297.2(\mathrm{CE}:-33 \mathrm{kV}), \mathrm{m} / \mathrm{z} 473.15\left(\mathrm{MH}^{+}\right)$to $122.15(\mathrm{CE}:-54 \mathrm{kV})$ and m/z $473.15\left(\mathrm{MH}^{+}\right)$to $160.1(\mathrm{CE}:-50 \mathrm{kV})$ were used to analyse noribogaine glucuronide. The MRMs for methadone were m/z $310.2\left(\mathrm{MH}^{+}\right)$to $265.15(\mathrm{CE}:-15 \mathrm{kV}), \mathrm{m} / \mathrm{z} 310.2\left(\mathrm{MH}^{+}\right)$to $105.05(\mathrm{CE}:-29 \mathrm{kV})$ and $\mathrm{m} / \mathrm{z} 310.2\left(\mathrm{MH}^{+}\right)$to $77.15(\mathrm{CE}:-50 \mathrm{kV})$. The MRMs for antipyrine were $\mathrm{m} / \mathrm{z} 189.1\left(\mathrm{MH}^{+}\right)$to $56.1(\mathrm{CE}:-35 \mathrm{kV}), \mathrm{m} / \mathrm{z} 189.1\left(\mathrm{MH}^{+}\right)$to $77.2(\mathrm{CE}:-42 \mathrm{kV})$ and m/z $189.1\left(\mathrm{MH}^{+}\right)$to $58.2(\mathrm{CE}:-23 \mathrm{kV})$. The MRMs were selected based on previous studies (Chang et al., 2011; Glue et al., 2016).

Mobile phase A was nanopure water containing $0.1 \%(\mathrm{v} / \mathrm{v})$ formic acid and mobile phase $\mathrm{B}$ was acetonitrile containing $0.1 \%(\mathrm{v} / \mathrm{v})$ formic acid. A gradient elution at a flow rate of 0.3 $\mathrm{ml} / \mathrm{min}$ was applied for the analysis with the program set as follows: the initial concentration was $100 \%$ mobile phase A, linearly changing to $100 \%$ mobile phase B over 7 min which was held for $1 \mathrm{~min}$. Then mobile phase B dropped to $0 \%$ over $1 \mathrm{~min}$ followed by equilibration of the system for 4 minutes. Total run time was 13 minutes. The injection volume was $1 \mu 1$ and the temperature of the column was kept at $40{ }^{\circ} \mathrm{C}$. The retention times for ibogaine, noribogaine, 
noribogaine glucuronide, methadone and antipyrine were 5.6, 5.0, 4.9, 6.3 and $5.1 \mathrm{~min}$, respectively, determined using commercially available reference compounds. Quantification was performed by comparing the respective peak areas of the total ion chromatogram (TIC) to the TIC peak areas of corresponding linear calibration curves of reference compounds $\left(\mathrm{R}^{2}>\right.$ 0.999), using Browser analysis in the software LabSolution (Shimadzu).

\subsection{Development of the PBK models}

As presented in Figure 3, a PBK model consisting of multiple organ compartments was developed to describe the ADME of ibogaine and its metabolite noribogaine upon oral administration. Noribogaine has also been reported to cause the prolongation effects on the QTc interval in human (Glue et al., 2016). Therefore, an oral administration route was included in the submodel of noribogaine, which enables modeling of noribogaine kinetics and prediction of its cardiotoxicity upon oral administration. Human physiological parameters reported in Brown et al. (1997) were used in the PBK model (Table 1).

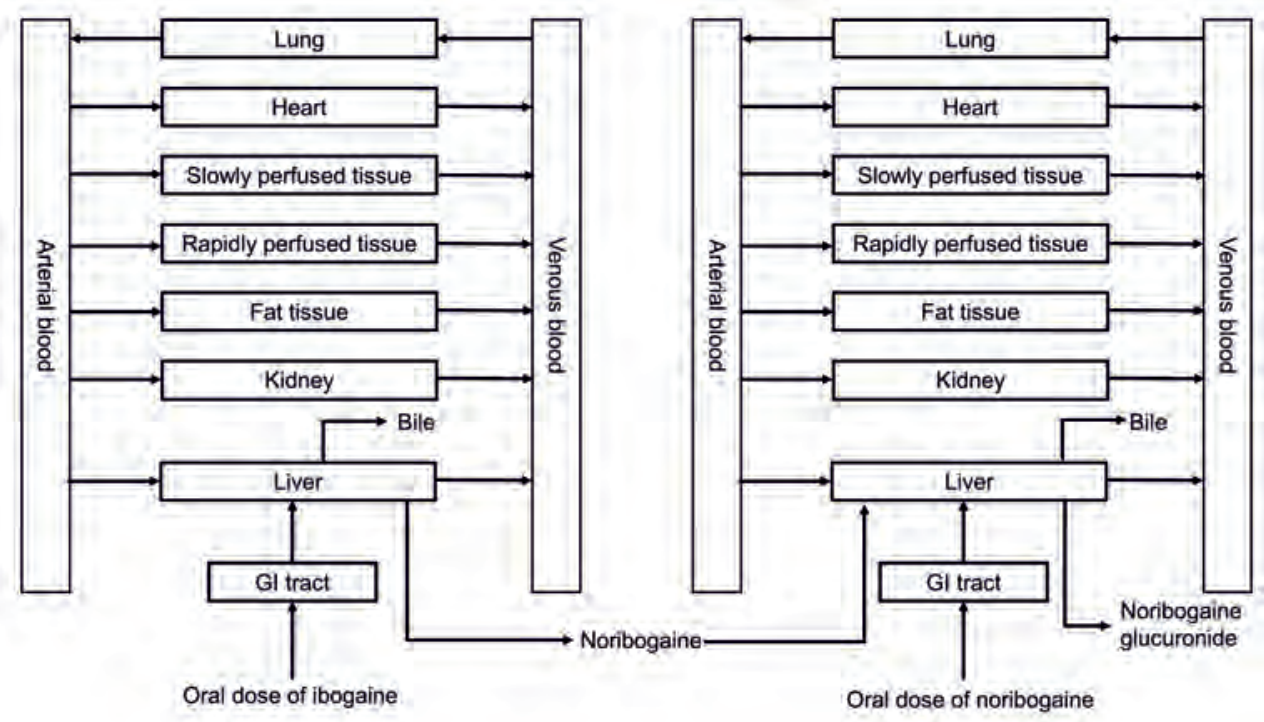

Figure 3 Schematic diagram of the PBK model for ibogaine with a submodel for noribogaine. 
Table 1 Physiological parameters used in the PBK models.

\begin{tabular}{lll}
\hline Parameters & $\begin{array}{l}\text { Symbol in model } \\
\text { code }\end{array}$ & Value $^{\mathrm{a}}$ \\
\hline Body weight (kg) & BW & 70 \\
Tissue volume (\% body weight) & & \\
Liver & VLc & 2.57 \\
Fat & VFc & 21.4 \\
Lung & VLuc & 0.76 \\
Arterial blood & VAc & 1.98 \\
Venous blood & VVc & 5.93 \\
Kidney & VKc & 0.4 \\
Heart & VHc & 0.47 \\
Slowly perfused tissue & VSc & 58 \\
Rapidly perfused tissue & VRc & 3.7 \\
Cardiac output (1/h) & Qc & 347.9 \\
Blood flow to tissue (\% cardiac output) & & \\
Liver & QLc & 22.7 \\
Fat & QFc & 5.2 \\
Kidney & QKc & 17.5 \\
Heart & QHc & 4 \\
Slowly perfused tissue & QSc & 29.1 \\
Rapidly perfused tissue & QRc & 21.5 \\
\hline Reported & & \\
\hline
\end{tabular}

${ }^{\mathrm{a}}$ Reported in Brown et al. (1997)

For the absorption parameters, the ka values of ibogaine and noribogaine were extrapolated from in vitro-derived $\mathrm{P}_{\text {app }}$ values obtained in the present study as described in the "in vitro intestinal transport studies" section. Due to limited pharmacokinetic data of both ibogaine and noribogaine, the experimental fractions absorbed $(\mathrm{Fa})$ were not available. However, many studies demonstrated a positive correlation between $\mathrm{P}_{\text {app }}$ values and human $\mathrm{Fa}$, and also indicated that Fa values can be estimated to be 1 when the $P_{\text {app }}$ value is higher than $10^{-5}(\mathrm{~cm} / \mathrm{s})$ (Lozoya-Agullo et al., 2015; Lüpfert and Reichel, 2005; Skolnik et al., 2010). Considering the relatively high $\mathrm{P}_{\text {app }}$ values measured for ibogaine and noribogaine (see Results), the Fa values for both compounds were assumed to be 1 . 
To describe how ibogaine and noribogaine distribute in organs and the systemic blood circulation upon absorption, tissue: blood partition coefficients $(\mathrm{P})$ of ibogaine and noribogaine were obtained by converting tissue: plasma partition coefficients using the corresponding blood/plasma ratio (BPr) as previously described (Shi et al., 2020a). The tissue: plasma partition coefficients were predicted using the QIVIVE tool (version 1.0) from Wageningen Food Safety Research (WFSR, 2020) in which the algorithm of Berezhkovskiy (2004) was applied for ibogaine and the algorithm of Rodgers and Rowland (2006) was used for noribogaine given it generally shows better prediction for zwitterions (Graham et al., 2012; Utsey et al., 2020). Other input parameters including acid-base properties (pKa), lipophilicity $(\log \mathrm{P})$ and fraction unbound in plasma $\left(\mathrm{f}_{\mathrm{u}, \mathrm{p}}\right)$. The $\log \mathrm{P}$ and $\mathrm{pKa}$ values were predicted using Chemicalize (ChemAxon, Hungary). The $\log \mathrm{P}$ and $\mathrm{pKa}$ of ibogaine were 3.53 and 8.97, respectively. The $\log \mathrm{P}$ and $\mathrm{pKa}$ of noribogaine were 3.0 and 8.87 (basic) and 9.66 (acidic). The $f_{u, p}$ values were determined using pooled human plasma in the present study. A BPr value of 2.5 for noribogaine in human was reported by Mash et al. (2016) while no published BPr value was available for ibogaine. Given that also for ibogaine the concentration was reported to be higher in the blood compared to plasma (Alper, 2001; Maciulaitis et al., 2008) the BPr value of ibogaine was assumed to be the same as that for noribogaine. Tissue: blood partition coefficients for ibogaine and noribogaine are summarized in Table 2.

Table 2 Tissue: blood partition coefficients for ibogaine and noribogaine.

\begin{tabular}{lcclllll}
\hline & \multicolumn{7}{c}{ Tissue: blood partition coefficients ${ }^{\text {a }}$} \\
\cline { 2 - 9 } Compound & liver & fat & $\begin{array}{l}\text { slowly } \\
\text { perfused tissue }\end{array}$ & $\begin{array}{l}\text { rapidly } \\
\text { perfused tissue }\end{array}$ & lung & kidney & heart \\
\hline Ibogaine & 1.62 & 0.18 & 2.73 & 1.62 & 0.32 & 1.02 & 0.7 \\
Noribogaine & 15.3 & 1.38 & 2.33 & 15.3 & 13.1 & 16.9 & 7.6 \\
\hline
\end{tabular}

${ }^{a}$ Obtained by dividing tissue: plasma partition coefficients by the BPr value.

Based on in vitro metabolism and in vivo pharmacokinetic studies, liver was considered as the major organ for the metabolism of ibogaine and noribogaine (Glue et al., 2016; Glue et al., 2015b; Obach et al., 1998). The kinetic parameters obtained in the current study were used to define the conversion of ibogaine to noribogaine and the glucuronidation of noribogaine by applying Michaelis-Menten kinetics. To extrapolate the in vitro $\mathrm{V}_{\max }$ to an in vivo $\mathrm{V}_{\max }$, a total microsomal protein per gram of liver (MPL) value of $32 \mathrm{mg} / \mathrm{g}$ was applied in the PBK model (Barter et al., 2007). The in vivo $\mathrm{K}_{\mathrm{m}}$ was assumed to be similar to the in vitro $\mathrm{K}_{\mathrm{m}}$. 
Hepatic metabolism was reported to be the major elimination route for ibogaine (Mash et al., 2016). For noribogaine, Glue et al. (2015a) found that only a small amount of the dose administered (1.4-3.9\%) was detected in urine as noribogaine and its glucuronide after a single oral dose of noribogaine in human, indicating the negligible contribution of urinary excretion to the elimination of noribogaine. For this reason renal excretion was not considered in the PBK model of noribogaine. Given the higher molecular weight of ibogaine and noribogaine than the cut-off value of $275 \mathrm{Da}$ for biliary excretion in human, the compounds could be excreted via bile instead of via urine (Haddad and Nong, 2020). This is supported by the fact that both ibogaine and noribogaine were detected in human bile (Kontrimavičiūtè et al. 2006; Maciulaitis et al. 2008) and were excreted via the gastrointestinal tract (Alper, 2001) and present in faeces in rat (Jeffcoat et al., 1993). Therefore, biliary excretion was assumed to be the major elimination route for ibogaine and noribogaine and was included in the PBK model. The biliary excretion rate constant $(\mathrm{kb})$ of noribogaine was obtained by the curve fitting option in Berkeley Madonna (version 8.3.18, UC Berkeley, CA, USA) in which the predicted blood maximum concentration $\left(\mathrm{C}_{\max }\right)$ of noribogaine was fitted to the $\mathrm{C}_{\max }$ of noribogaine in the blood that was reported in clinical studies (Glue et al., 2016; Glue et al., 2015a; Glue et al., 2015b). The averaged fitted $\mathrm{kb}$ for noribogaine was $0.575(/ \mathrm{h})$. Due to the limited pharmacokinetic data on ibogaine and little influence of biliary excretion on ibogaine blood kinetics (see the results of the sensitivity analysis), the same $\mathrm{kb}$ value was assumed for ibogaine. Kinetic model calculations and curve fitting were performed with Berkeley Madonna, applying Rosenbrock's algorithms for solving stiff systems. Model equations are shown in Supplementary materials 2.

\subsection{Evaluation of the PBK model}

To evaluate the model, the predicted blood concentrations and area under the blood concentration- time curve (AUC) of ibogaine and noribogaine were compared with the in vivo data reported in clinical studies (Glue et al., 2016; Glue et al., 2015a; Glue et al., 2015b). The reported plasma-based kinetics of ibogaine and noribogaine were extracted using WebPlotDigitizer Version 4.4. (Rohatgi, 2020) and converted to blood-based kinetics by multiplying with the respective BPr values. The evaluation was performed according to the specifications (body weight and oral dose) of in vivo studies as summarized in Table 4.

\subsection{Sensitivity analysis}

A local parameter sensitivity analysis was conducted to estimate to what extent the model parameters can influence the model output, which refers to $\mathrm{C}_{\max }$ of ibogaine and noribogaine 
in the heart venous blood upon the oral administration of ibogaine or noribogaine. Furthermore, given that the in vivo cardiotoxicity of ibogaine is dependent on the unbound concentration of both ibogaine and noribogaine, the sensitivity analysis was also performed for the unbound toxic equivalence (TEQ) concentration (details see in "QIVIVE using PBK modeling-based reverse dosimetry" section). The sensitivity coefficient (SC) was calculated according to the Eq. 5:

$$
\mathrm{SC}=\frac{\left(\mathrm{C}^{\prime}-\mathrm{C}\right)}{\left(\mathrm{P}^{\prime}-\mathrm{P}\right)} \times \frac{\mathrm{P}}{\mathrm{C}}
$$

where $\mathrm{P}$ and $\mathrm{C}$ represent the initial value of the model parameter and output, respectively. $\mathrm{P}$ ' and C' stand for the model parameter and model output after a $1 \%$ increase in an individual model parameter value, respectively. Only parameters with an absolute $\mathrm{SC}>0.1$ are considered to be influential on the model output (Rietjens et al. 2011). The sensitivity analysis was carried out for a subject with a body weight of $70 \mathrm{~kg}$ (Brown et al., 1997) and for a single oral dose of 20 and $500 \mathrm{mg}$ ibogaine, representing a safe and well tolerated dose for healthy people (Glue et al., 2015b) and a clinically relevant dose for the treatment of drug addiction (Maciulaitis et al., 2008), respectively. For the sensitivity analysis of the noribogaine model, a single oral dose of $20 \mathrm{mg}$ and $200 \mathrm{mg}$ was chosen, respectively representing a safe dose for healthy people and a dose level associated with prolonged QTc in human (Glue et al., 2016).

\subsection{QIVIVE using PBK modeling-based reverse dosimetry}

Given that the in vitro endpoint FPDc can be considered as a surrogate endpoint for the QTc interval in the human ECG (Shi et al., 2020a; Zwartsen et al., 2019), in vitro concentrationresponse curves for FPDc obtained in the hiPSC-CM MEA assay were translated to in vivo dose-response curves for QTc using PBK modeling-based reverse dosimetry. In the case of oral administration of ibogaine, a TEQ approach was applied to combine the cardiotoxicity of ibogaine and noribogaine. Assuming that the cardiotoxicity of ibogaine and noribogaine are additive to hiPSC-CM, the internal unbound TEQ concentration was the combination of the unbound concentration of ibogaine and noribogaine in the heart venous blood taking the corresponding toxic equivalency factors (TEFs) into account. The TEF of ibogaine and noribogaine were calculated based on their cardiotoxic potencies obtained in the hiPSC-CM MEA assay, with the TEF for ibogaine defined as 1.00. Then QIVIVE was performed by assuming the in vitro unbound concentration of ibogaine equal to the unbound $C_{\max }$ of unbound 
ibogaine expressed in TEQ concentrations in the heart venous blood as shown in the Eq. 6 and 7:

$\mathrm{C}_{\text {total, in vitro, ibo }} \mathrm{X}_{\mathrm{u}, \mathrm{m} \text {, ibo }}=\mathrm{C}_{\text {unbound, human blood, } \mathrm{TEQ}}$

$\mathrm{C}_{\text {unbound, human blood, TEQ }}=\mathrm{C}_{\text {total, human blood, ibo }} \times \frac{\mathrm{f}_{\mathrm{u}, \mathrm{p}, \text { ibo }}}{\mathrm{BPr}_{\mathrm{ibo}}} \times \mathrm{TEF}_{\text {ibo }}+$

$\mathrm{C}_{\text {total, human blood,nor }} \mathrm{x} \frac{\mathrm{f}_{\mathrm{u}, \mathrm{p} \text {,nor }}}{\mathrm{BPr} \text { nor }} \times \mathrm{TEF}_{\text {nor }}$

where $\mathrm{C}_{\text {total, in vitro,ibo }}$ and $\mathrm{f}_{\mathrm{u}, \mathrm{m}}$, ibo are the in vitro ibogaine concentration and unbound fraction of ibogaine in the in vitro exposure medium, respectively. $\mathrm{BPr}_{\mathrm{ibo}}$ and $\mathrm{BPr}_{\text {nor }}$ are the blood to plasma ratio of ibogaine and noribogaine. $f_{u, p}$, ibo and $f_{u, p}$, nor are the respective unbound fraction of ibogaine and noribogaine in human plasma. $\mathrm{C}_{\text {total, human blood, ibo and }}$ $\mathrm{C}_{\text {total, human blood, nor }}$ are the concentrations of ibogaine and noribogaine in the heart venous blood, respectively. $\mathrm{TEF}_{\mathrm{ibo}}$ and $\mathrm{TEF}_{\text {nor }}$ are the $\mathrm{TEF}$ values of ibogaine (defined as 1.00) and noribogaine (defined based on its relative potency in the MEA assay). $\mathrm{C}_{\text {unbound, human blood, TEQ }}$ values were converted to in vivo oral doses of ibogaine by PBK-modeling based reverse dosimetry, using a bodyweight of $70 \mathrm{~kg}$ (Brown et al., 1997).

When oral administration of noribogaine was considered, the translation was performed by setting the in vitro unbound concentrations of noribogaine detected in the hiPSC-CM MEA assay equal to the unbound $\mathrm{C}_{\max }$ of noribogaine in the heart venous blood with a correction for the fraction unbound in human plasma and conversion from plasma to blood using BPr as described in in Eq.8:

$$
\mathrm{C}_{\text {total, human blood, nor }}=\frac{\mathrm{C}_{\text {total, in vitro, nor }} \mathrm{x}_{\mathrm{u}, \mathrm{m}, \text { nor }}}{\frac{\mathrm{f}_{\mathrm{u}, \mathrm{p}, \text { nor }}}{\mathrm{BPr} \text { nor }}}
$$

Where $\mathrm{C}_{\text {total, in vitro,nor }}$ and $\mathrm{f}_{\mathrm{u}} \mathrm{m}$, nor are the in vitro noribogaine concentration and unbound fraction of noribogaine in the in vitro exposure medium, respectively. $\mathrm{C}_{\text {total, human blood, nor }}$ values were extrapolated to in vivo oral doses of noribogaine by PBK-modeling based reverse dosimetry, using a bodyweight of $70 \mathrm{~kg}$ (Brown et al., 1997). The calculations by Eq. 6 and 7 or 8 were performed for each of the in vitro concentrations of ibogaine or noribogaine tested in the hiPSC-CM MEA assay, which enables the translation of the entire in vitro concentrationresponse curve to a predicted in vivo dose-response curve. 


\subsection{Validation of the PBK modeling-based reverse dosimetry approach}

To validate the performance of the PBK modeling-based reverse dosimetry approach, the predicted dose-response curves for QTc prolongation upon the exposure to ibogaine and noribogaine were compared to the respective in vivo dose-response data obtained from single case reports and clinical studies (Asua, 2013; Glue et al., 2016; Grogan et al., 2019; Henstra et al., 2017; Hildyard et al., 2016; Hoelen et al., 2009; Meisner et al., 2016; Pleskovic et al., 2012; Steinberg and Deyell, 2018; Vlaanderen et al., 2014). Since in most case reports ibogaine administered to the patients were internet-purchased with unknown purity, the reported doses were converted to effective doses by multiplying with the lower (15\%) and upper value $(50 \%)$ of purity reported in Alper et al. (2012). The predicted dose-response curve of noribogaine for the validation was made using the bodyweight of $81.9 \mathrm{~kg}$, which was the average body weight of subjects as reported in Glue et al. (2016). The details of the in vivo studies are summarized in Table S1 in the supplementary material 1. The in vitro absolute FPDc values and the in vivo QTc data were expressed as relative percentages by comparing the post-treatment data to the baseline values for a straightforward comparison.

\subsection{Benchmark dose analysis}

BMD analysis of reported and predicted dose-response curves for ibogaine and noribogaine was performed to derive a lower $95 \%$ confidence limit of the BMD resulting in $10 \%$ cardiotoxicity $\left(\mathrm{BMDL}_{10}\right)$, which can be used as the point of departure (PoDs) for the risk assessment and safety evaluation of ibogaine and noribogaine. As previously described (Shi et al., 2020a), an effective size of $10 \%$ was chosen based on the fact that a BMDL 10 value is generally considered as a dose level similar to a no observed adverse effect level (EFSA, 2017), and that $10 \%$ change in QTc interval over the population baseline of $407 \mathrm{~ms}$, being a QTc of $450 \mathrm{~ms}$ can be used as a threshold to evaluate the abnormal QTc prolongation (ICH 2005; Mujtaba et al. 2013; Wedam et al. 2007; Florian et al. 2012). The European Food Safety Authority web-tool based on R-package PROAST version 69 (Dutch National Institute for Public Health and the Environment, RIVM, The Netherlands) was used for the BMD analysis (Shi et al., 2020a).

BMD analysis of in vitro concentration-response data was performed to calculate the benchmark concentrations (BMC) resulting in 10\% change in the FPDc with lower 95\% confidence limit $\left(\mathrm{BMCL}_{10}\right)$. The obtained $\mathrm{BMCL}_{10}$ values were used to compare the potency and derive the TEF of noribogaine relative to the TEF of ibogaine set at 1.00. 


\section{Results}

\subsection{In vitro cardiotoxicity of ibogaine and noribogaine in the hiPSC-CM}

\section{MEA assay}

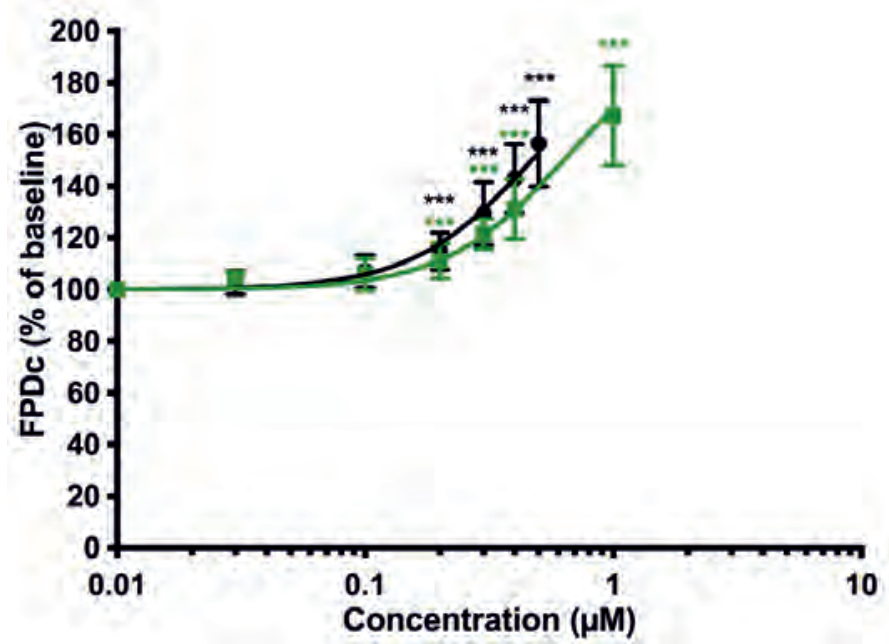

Figure 4 Concentration-response curves for the effect of ibogaine (black circles and line) and noribogaine (green squares and line) on FPDc in hiPSC-CM detected by the MEA. The response of the baseline condition $(0.05 \%(\mathrm{v} / \mathrm{v})$ acetonitrile for ibogaine, $0.1 \%(\mathrm{v} / \mathrm{v})$ DMSO for noribogaine) was set at $100 \%$. Data represent the mean of results obtained from three independent experiments each containing six well replicates. Each data point represents the mean \pm SD. Statistically significant changes in response compared to the solvent control are marked with ${ }^{*}$ with $p<0.001:{ }^{* * *}$.

Figure 4 shows that ibogaine and its metabolite noribogaine induced a significant concentration-dependent prolongation of FPDc. The $\mathrm{BMCL}_{10}$ is $0.11 \mu \mathrm{M}$ for ibogaine and 0.15 $\mu \mathrm{M}$ for noribogaine, which results in TEF values of ibogaine and noribogaine being 1.00 and 0.73 , respectively. Arrhythmia-type waveforms were observed upon the treatment with $1 \mu \mathrm{M}$ ibogaine and $3 \mu \mathrm{M}$ noribogaine. The repeated addition of vehicle controls $(0.05 \%(\mathrm{v} / \mathrm{v})$ acetonitrile and $0.1 \%(\mathrm{v} / \mathrm{v})$ DMSO) did not significantly influence the FPDc (Figure S2). Two reference compounds dofetilide and isoproterenol respectively prolonged the $\mathrm{FPD}_{\mathrm{C}}$ and increased beat rates in a concentration-dependent manner, indicating the adequate performance of the hiPSC-CM MEA assay (Figure S3). 


\subsection{In vitro experimental biokinetic parameters for PBK models}

\subsubsection{In vitro intestinal transport studies}

Table 3 shows the $\mathrm{P}_{\text {app }}$ values obtained from Caco- 2 transport studies and the ka values of the test compounds derived from these $\mathrm{P}_{\text {app }}$ values based on comparison to the data for methadone. All test compounds were rapidly transported with the largest $P_{\text {app }}$ value of $47.0 \times 10^{-6} \mathrm{~cm} / \mathrm{s}$ for antipyrine and the smallest $\mathrm{P}_{\text {app }}$ value of $20.8 \times 10^{-6} \mathrm{~cm} / \mathrm{s}$ for methadone. The $\mathrm{P}_{\text {app }}$ value of noribogaine was 1.5 -fold higher than that of ibogaine. The transport of all test compounds was within the linear range up to $30 \mathrm{~min}$ of incubation. The mass recovery of ibogaine, noribogaine, methadone and antipyrine are $90 \%, 102 \%, 74 \%$ and $88 \%$, respectively.

Table 3 The apparent permeability $\left(P_{\text {app }}\right)$ obtained from Caco-2 transport studies, and the predicted intestinal oral absorption rate constants (ka) for the test compounds derived from the $P_{\text {app }}$ values using the ka for methadone (Foster et al., 2000; Wolf et al., 2000) as the reference.

\begin{tabular}{lll}
\hline Compound & Papp $\pm \operatorname{SD}\left(10^{-6} \mathrm{~cm} / \mathrm{s}\right)$ & $\mathrm{ka}(/ \mathrm{h})$ \\
\hline Ibogaine & $27.9 \pm 4.6$ & 0.79 \\
Noribogaine & $42.4 \pm 3.6$ & 1.23 \\
Methadone & $20.8 \pm 1.9$ & $0.59^{\mathrm{a}}$ \\
Antipyrine & $47.0 \pm 5.0$ & 1.33 \\
\hline
\end{tabular}

${ }^{a}$ Reported value obtained from human studies (Foster et al., 2000; Wolf et al., 2000)

\subsubsection{In vitro microsomal incubations}

Figure 5 shows the substrate concentration dependent metabolism of ibogaine and noribogaine by human liver microsomes. The obtained results follow Michaelis-Menten kinetics. The apparent $\mathrm{V}_{\max }$ and $\mathrm{K}_{\mathrm{m}}$ derived from these data for the formation of noribogaine from ibogaine (Figure 5a) were $0.17 \pm 0.033 \mathrm{nmol} / \mathrm{min} / \mathrm{mg}$ microsomal protein and $0.63 \pm 0.005 \mu \mathrm{M}$, respectively. The apparent $\mathrm{V}_{\max }$ and $\mathrm{K}_{\mathrm{m}}$ for the conversion of noribogaine to its glucuronide (Figure $5 \mathrm{~b}$ ) were $0.036 \pm 0.0008 \mathrm{nmol} / \mathrm{min} / \mathrm{mg}$ microsomal protein and $305 \pm 15.8 \mu \mathrm{M}$, respectively. The catalytic efficiency $\left(\mathrm{V}_{\max } / \mathrm{K}_{\mathrm{m}}\right)$ for the formation of noribogaine was 269.8 $\mu 1 / \mathrm{min} / \mathrm{mg}$ microsomal protein, which was 2,248-fold more efficient than that for formation of noribogaine glucuronide being $0.12 \mu \mathrm{l} / \mathrm{min} / \mathrm{mg}$ microsomal protein. This explains the relatively higher plasma concentrations of noribogaine than of ibogaine upon dosing ibogaine (see below). 

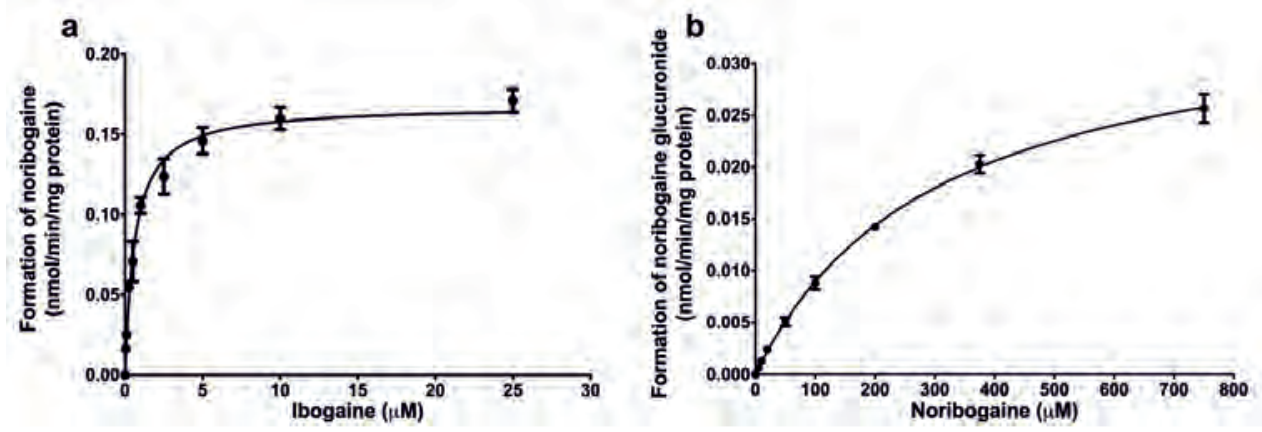

Figure 5 Concentration-dependent formation of (a) noribogaine from ibogaine and (b) noribogaine glucuronide from noribogaine using in vitro incubations with human liver microsomes. Data represent the mean of three independent experiments. Each data point represents the mean \pm SD.

\subsection{Determination of plasma protein and in vitro medium binding}

The unbound fraction of ibogaine and noribogaine in the in vitro medium and human plasma were determined by the RED assay, in order to enable the calculation of the unbound concentration of the compounds for the in vitro to in vivo extrapolation. The unbound fraction of ibogaine and noribogaine in the in vitro medium were comparable, amounting to $0.71 \pm 0.01$ and $0.80 \pm 0.03$, respectively. The unbound fraction of ibogaine in human plasma was determined to be $0.04 \pm 0.017$, which was 6.5 -fold lower than that for noribogaine, being 0.26 \pm 0.05 .

\subsection{Evaluation of the PBK model}

The PBK models of ibogaine and noribogaine were evaluated against in vivo data reported in clinical studies. Figure 6 shows that the developed PBK model accurately predicted the timedependent change in the blood concentrations of ibogaine and noribogaine upon oral administration of ibogaine (Glue et al., 2015b) and noribogaine (Glue et al., 2015a; Glue et al., 2016). The detailed comparisons between predicted and reported blood $\mathrm{C}_{\max }$ and AUC values are summarized in Table 4. For ibogaine, the predicted blood $\mathrm{C}_{\max }$ and AUC were 1.7- fold and 2.1-fold higher than the reported values, respectively. For noribogaine, the prediction shows an average 0.9 -fold and 1.3-fold difference both in blood $\mathrm{C}_{\max }$ and $\mathrm{AUC}$ values. 
Table 4 Summary of in vivo kinetic studies and evaluation of the PBK model predictions for ibogaine and noribogaine blood $\mathrm{C}_{\max }$ and AUC.

\begin{tabular}{|c|c|c|c|c|c|c|c|c|c|}
\hline $\begin{array}{l}\text { Compou } \\
\text { nd }\end{array}$ & $\begin{array}{l}\text { Mean } \\
\text { body } \\
\text { weight } \\
(\mathrm{kg})\end{array}$ & $\begin{array}{l}\text { Mean } \\
\text { dose } \\
(\mathrm{mg} / \text { day } \\
)\end{array}$ & $\begin{array}{l}\text { In vivo } \\
\mathrm{C}_{\max } \\
(\mathrm{ng} / \mathrm{ml})^{\mathrm{a}}\end{array}$ & $\begin{array}{l}\text { Predicted } \\
\mathrm{C}_{\max } \\
(\mathrm{ng} / \mathrm{ml})\end{array}$ & $\begin{array}{l}\text { Ratio } \\
\text { predicted } \\
\mathrm{C}_{\max } / \text { in } \\
\text { vivo } \mathrm{C}_{\max }\end{array}$ & $\begin{array}{l}\text { In vivo } \\
\text { AUC } \\
\left(\mathrm{ng}{ }^{*} \mathrm{~h} / \mathrm{ml}\right) \\
\mathrm{a}\end{array}$ & $\begin{array}{l}\text { Predicted } \\
\text { AUC } \\
\left(\mathrm{ng}^{*} \mathrm{~h} / \mathrm{ml}\right)\end{array}$ & $\begin{array}{l}\text { Ratio } \\
\text { predicted } \\
\text { AUC/in } \\
\text { vivo } \\
\text { AUC }\end{array}$ & $\begin{array}{l}\text { Referenc } \\
\mathrm{e}\end{array}$ \\
\hline \multirow{2}{*}{$\begin{array}{l}\text { Ibogaine } \\
\text { Noribog } \\
\text { aine }\end{array}$} & \multirow[b]{2}{*}{$80^{\mathrm{b}}$} & 20 & 2.75 & 4.81 & 1.7 & 9.0 & 19.3 & 2.1 & \multirow{2}{*}{$\begin{array}{l}\text { Glue et } \\
\text { al. } \\
(2015 b)\end{array}$} \\
\hline & & $-d$ & 46.8 & 54.9 & 1.2 & 693.5 & 1029.4 & 1.5 & \\
\hline \multirow{4}{*}{$\begin{array}{l}\text { Noribog } \\
\text { aine }\end{array}$} & \multirow{4}{*}{78} & 3 & 13.0 & 10.0 & 0.77 & 185.5 & 165.8 & 0.89 & \multirow{4}{*}{$\begin{array}{l}\text { Glue et } \\
\text { al. } \\
\text { (2015a) }\end{array}$} \\
\hline & & 10 & 36.3 & 33.5 & 0.92 & 636.25 & 552.8 & 0.87 & \\
\hline & & 30 & 139.8 & 100.4 & 0.72 & 1751.0 & 1658.4 & 0.95 & \\
\hline & & 60 & 290.0 & 200.8 & 0.69 & 4905.5 & 3316.9 & 0.68 & \\
\hline \multirow{3}{*}{$\begin{array}{l}\text { Noribog } \\
\text { aine }\end{array}$} & & 60 & 204.0 & 191.3 & 0.94 & 5150.8 & 3924.1 & 0.76 & \multirow{3}{*}{$\begin{array}{l}\text { Glue et } \\
\text { al. (2016) }\end{array}$} \\
\hline & 81.9 & 120 & 432.0 & 382.7 & 0.89 & 8201.3 & 7848.5 & 0.96 & \\
\hline & & 180 & 669.8 & 574.1 & 0.86 & 17219.3 & 11773.3 & 0.68 & \\
\hline
\end{tabular}

${ }^{a}$ Blood data were obtained by multiplying reported plasma data by the BPr value.

${ }^{\mathrm{b}}$ The body weight was not reported and set equal to the average of values in other studies conducted by the same group (Glue et al., 2016; Glue et al., 2015a), assuming subjects have similar demographic characteristics.

${ }^{\mathrm{c}}$ The body weight was not reported and set equal to the value used in the PBK model.

${ }^{\mathrm{d}}$ Subjects were administered ibogaine. 


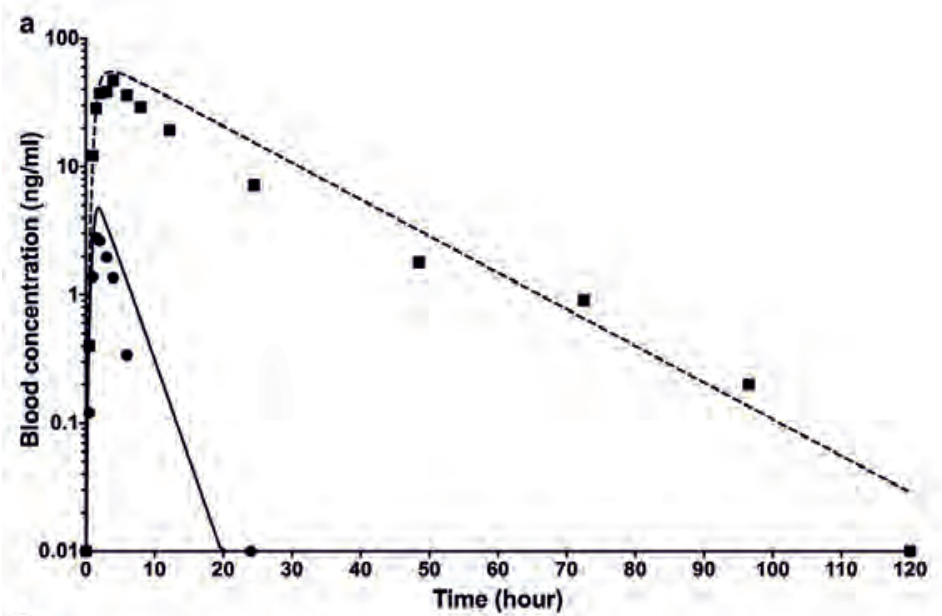

b

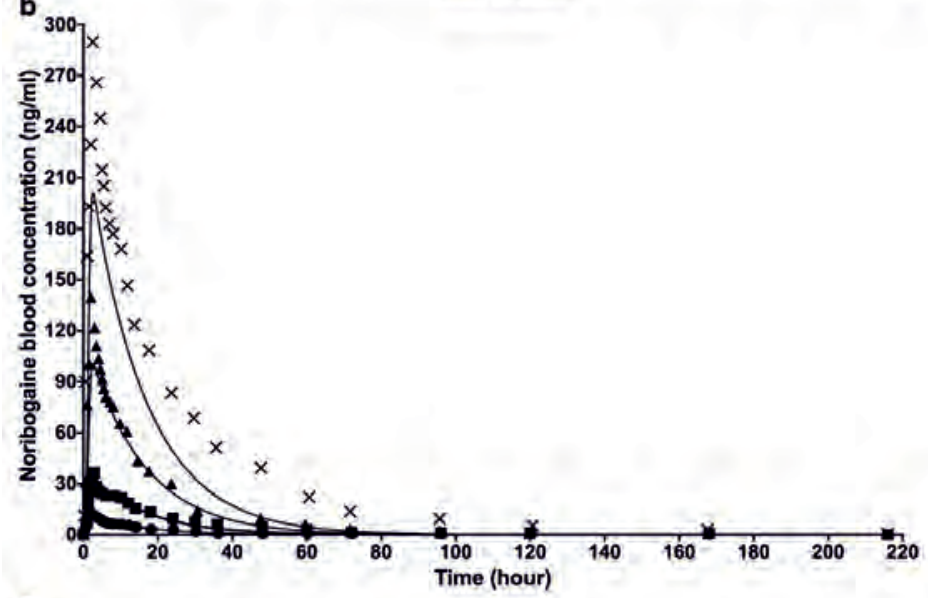

c

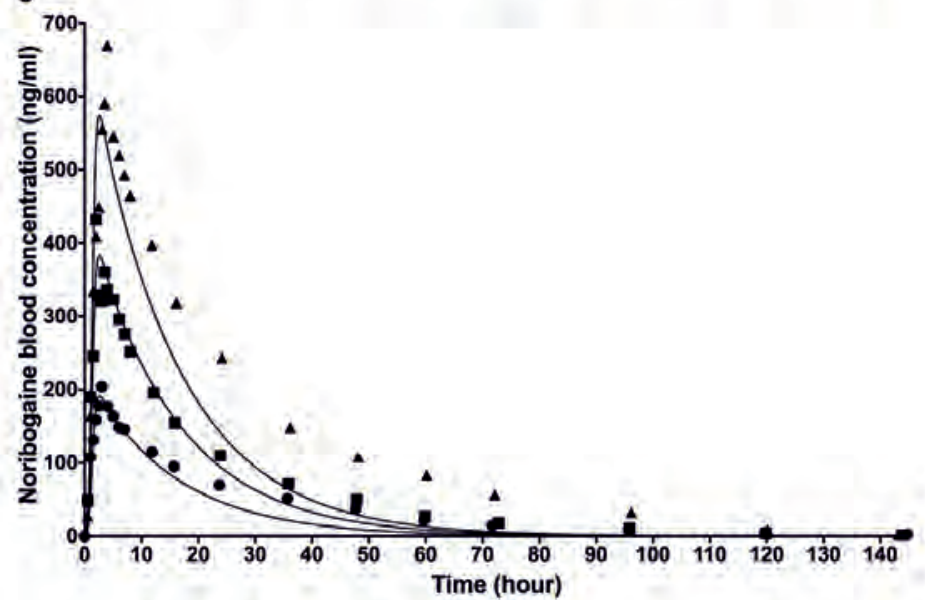


Figure 6 Comparison of blood concentration-time curves of ibogaine and noribogaine in human predicted with the PBK model and as published in the literature for human case studies. (a) Dots and squares respectively indicate the reported blood concentrations of ibogaine and noribogaine after a single oral dose of $20 \mathrm{mg}$ ibogaine (Glue et al., 2015b). Solid lines and dashed lines represent the predictions for ibogaine and noribogaine, respectively. (b) Dots, squares, triangles and crosses represent the reported blood concentrations after an oral dose of 3, 10, 30 and $60 \mathrm{mg}$ noribogaine (Glue et al., 2015a), respectively, with the solid lines being the predicted blood concentrations for the corresponding doses. (c) Dots, squares, and triangles represent the reported blood concentrations after an oral dose of 60, 120 and 180 noribogaine (Glue et al., 2016), respectively, with the solid lines being the predicted blood concentrations of the corresponding doses.

\subsection{Sensitivity analysis}
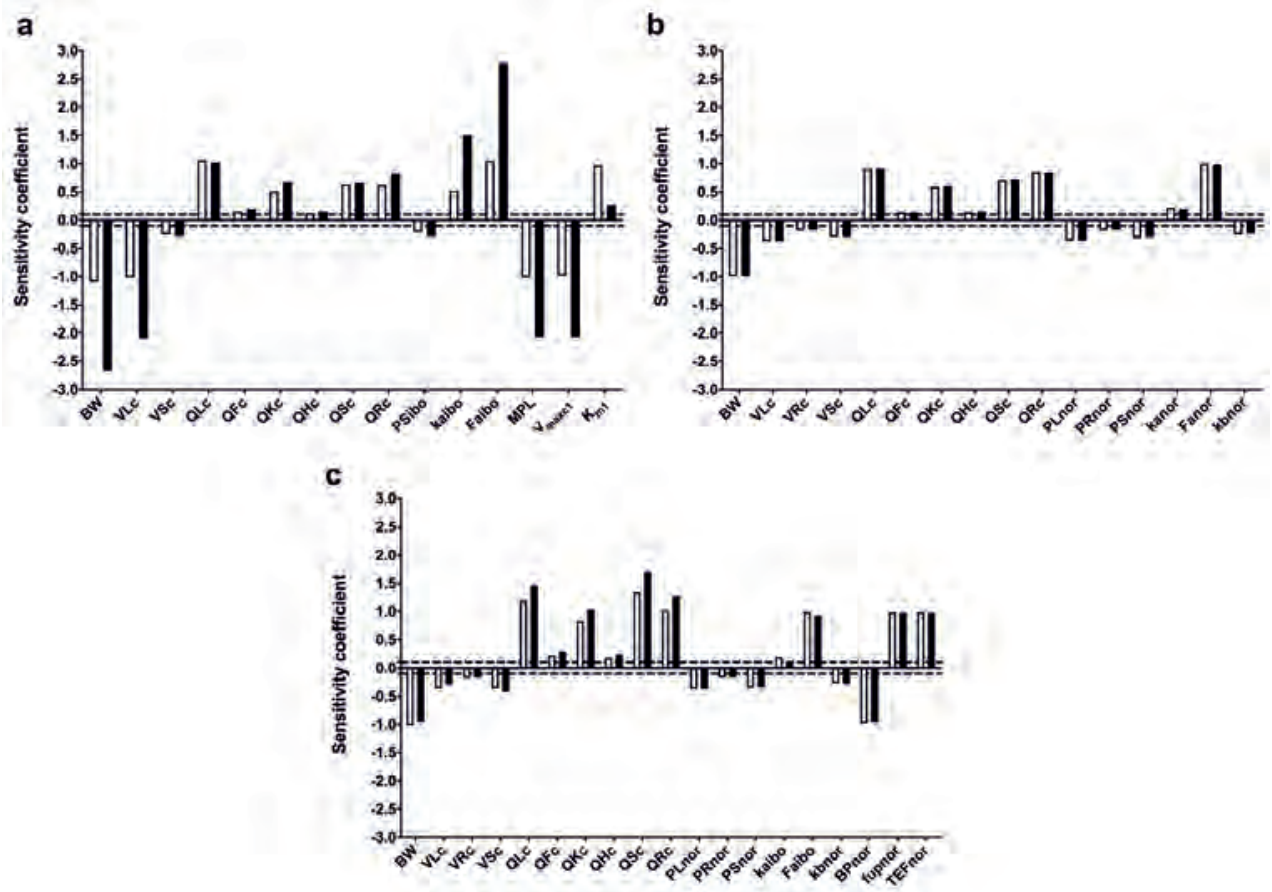

Figure 7 Sensitivity coefficients of PBK model parameters for the prediction of (a) $C_{\max }$ of ibogaine in the heart venous blood upon an oral single ibogaine dose of $20 \mathrm{mg}$ (white bars) and $500 \mathrm{mg}$ (black bars), (b) $\mathrm{C}_{\max }$ of noribogaine in the heart venous blood upon an oral single noribogaine dose of $20 \mathrm{mg}$ (white bars) and $200 \mathrm{mg}$ (black bars) and (c) $\mathrm{C}_{\max }$ expressed in unbound ibogaine equivalents upon an oral single ibogaine dose of $20 \mathrm{mg}$ (white bars) and 500 mg (black bars). Dotted lines indicate the normalized SC with an absolute value higher than 0.1. BW, body weight; VLc, fraction of liver; VRs, fraction of rapidly perfused tissue; VSc, fraction of slowly 
perfused tissue; QLc, percentage of blood flow to liver; QKc, percentage of blood flow to kidney; QHc, percentage of blood flow to heart; QRc, percentage of blood flow to rapidly perfused tissue; QSc, percentage of blood flow to slowly perfused tissue; PSibo, partition coefficient slowly perfused tissue: blood of ibogaine; MPL, microsomal protein per gram of liver; $\mathrm{V}_{\operatorname{maxc}}$, unscaled maximum rate of ibogaine metabolism in liver; $\mathrm{K}_{\mathrm{m} 1}$, Michaelis-Menten constant for ibogaine metabolism in liver; PLnor, partition coefficient liver: blood of noribogaine; PSnor, partition coefficient slowly perfused tissue: blood of noribogaine; kaibo, absorption rate constant of ibogaine; kanor, absorption rate constant of noribogaine; Faibo, fraction absorbed of ibogaine; Fanor, fraction absorbed of noribogaine; Kbnor, biliary excretion constant of noribogaine; BPnor, blood to plasma ratio of noribogaine; fupnor, unbound fraction of noribogaine in human plasma; TEFnor, toxic equivalency factor of noribogaine.

Figure 7 shows the results of the sensitivity analysis presenting the influential model parameters for the prediction of $\mathrm{C}_{\max }$ of ibogaine and noribogaine in the heart venous blood and of the $\mathrm{C}_{\max }$ expressed in unbound ibogaine equivalents using a TEQ approach, upon exposure to an oral dose of ibogaine or noribogaine. For the oral administration of ibogaine (Figure $7 \mathrm{a}$ ), results reveal that $\mathrm{C}_{\max }$ of ibogaine in the heart venous is most sensitive to the body weight, fraction absorbed of ibogaine, fraction of liver, percentage of blood to liver and metabolic parameters for conversion of ibogaine to noribogaine (MPL, $\mathrm{V}_{\operatorname{maxc1}}$ and $\mathrm{K}_{\mathrm{m} 1}$ ). When the oral dose increased from $20 \mathrm{mg}$ to $500 \mathrm{mg}$, the normalized SC values of body weight, fraction of liver, absorption related parameters (Faibo and kaibo) and metabolic parameters (MPL and $\mathrm{V}_{\operatorname{maxc}}$ ) increased 2- to 3-fold while the normalized $\mathrm{SC}$ values of $\mathrm{K}_{\mathrm{m} 1}$ shows a 3.6fold decrease.

As illustrated in Figure $7 \mathrm{~b}$ similar SC values were obtained for the prediction of the $\mathrm{C}_{\max }$ of noribogaine in the heart venous blood at two oral doses of $20 \mathrm{mg}$ and $200 \mathrm{mg}$ noribogaine. The predicted $\mathrm{C}_{\max }$ of noribogaine in the heart venous blood is most affected by the fraction absorbed of noribogaine and the body weight with the normalized SC values being 1 . Parameters related to percentage of blood to tissues also influence the prediction especially the percentage of blood to liver, rapidly perfused tissue, slowly perfused tissue and kidney with the normalized SC values above 0.5. Other model parameters show less influence with the normalized SC values ranging from 0.14 to 0.37 (Figure $7 b$ ).

Figure 7c shows that the unbound TEQ concentration expressed in ibogaine equivalents is most sensitive to the percentage of blood flow to slowly perfused tissue, followed by the percentage of blood flow to liver, and to rapidly perfused tissue with normalized SC values above 1 . Besides, body weight, fraction absorbed of ibogaine, blood to plasma ratio of 
noribogaine, unbound fraction of noribogaine in plasma and TEF of noribogaine show a high influence on the prediction with the normalized $\mathrm{SC}$ values being 1 . Figure $7 \mathrm{c}$ also indicates that parameters related to percentage of blood flow to tissues (QSc, QLc, QRc, QKc, QFc and QHc) show a dose-dependent influence on the prediction with the normalized SC values being higher at $500 \mathrm{mg}$ compared to those at $20 \mathrm{mg}$. While the SC of other model parameters generally are not dose-dependent at the two doses of ibogaine.

\subsection{Contribution of ibogaine and noribogaine to blood ibogaine equivalents}

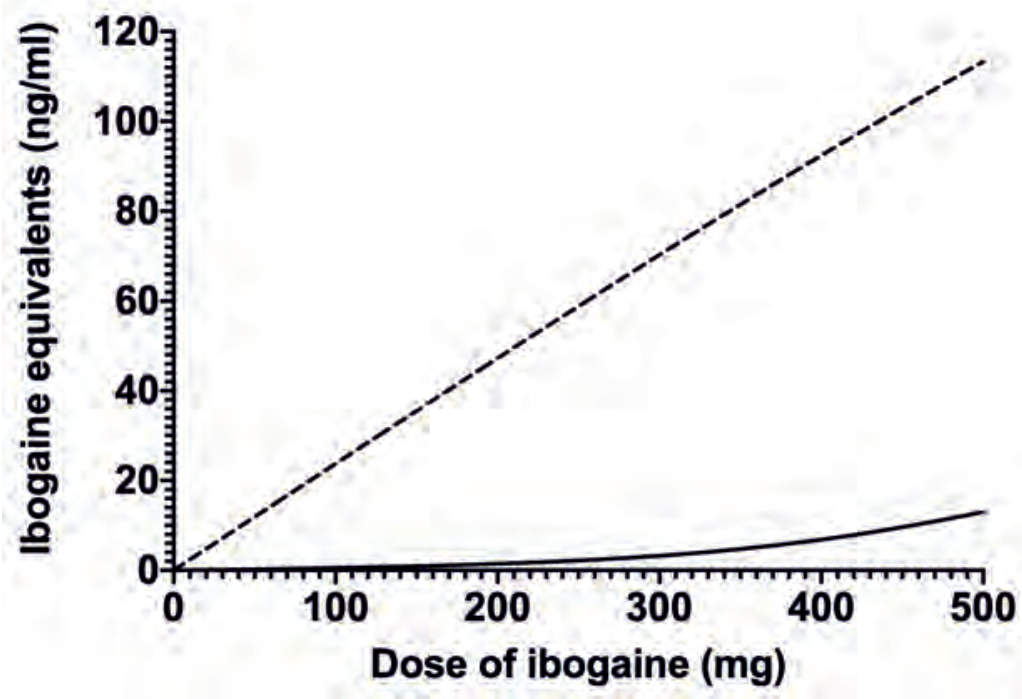

Figure 8 Predicted dose-dependent relative contribution of ibogaine (solid line) and noribogaine (dashed line) to the $C_{\max }$ expressed in unbound ibogaine equivalents for a human of $70 \mathrm{~kg}$.

To further illustrate the contribution of ibogaine and noribogaine to ibogaine-induced cardiotoxicity in human, a dose-dependent comparison was made between the predicted unbound blood concentration of ibogaine and noribogaine taking into account their TEF values. Figure 8 shows that, upon dosing ibogaine, the contribution of noribogaine to the $C_{\max }$ expressed in unbound ibogaine equivalents is substantially higher than the contribution of ibogaine itself at all dose levels evaluated. The relative contribution of ibogaine to the ibogaine equivalents increases with increasing oral dose, but is still about 9-fold lower than that of noribogaine at $500 \mathrm{mg}$, in spite of the only limited difference in the TEF value between the two compounds. This observation can be ascribed to the fact that the concentration of noribogaine 
is higher than that of ibogaine (see also Figure 6a) due to the fact the catalytic efficiency of ibogaine O-demethylation to noribogaine is more efficient than the clearance of noribogaine by glucuronidation.

\subsection{QIVIVE using PBK modeling-based reverse dosimetry, its validation and BMD analysis}

By applying reverse dosimetry, the in vitro concentration-response curves of ibogaine and noribogaine obtained in the hiPSC-CM MEA assay were translated to predicted in vivo doseresponse curves for the QTc prolongation, upon oral administration of ibogaine (Figure 9a) or noribogaine (Figure 9b). Subsequently, the predicted data were compared to the in vivo doseresponse data for QTc prolongation obtained from case studies and clinical studies to evaluate the performance of the PBK modeling-based reverse dosimetry predictions (Figure 9). Given the unknown purity of internet-purchased ibogaine described in the case studies, a range of 15 to $50 \%$ was used to correct for the effective doses. Figure 9 a reveals that the predicted dosedependent QTc prolongation for ibogaine is best in line with the reported data when the a purify of $15 \%$ was considered. For noribogaine, the predicted dose-response curve is comparable with the reported dose-response data on QTc prolongation (Figure 9b).

To further evaluate the model predictions and derive PoDs for risk assessment, $\mathrm{BMDL}_{10}$ values were calculated from both predicted and reported dose-response curves. The BMDL 10 value of noribogaine derived from the clinical study of Glue et al. (2016) was $163 \mathrm{mg}$ for the subjects with an average body weight of $81.9 \mathrm{~kg}$, which is 1.5 -fold higher than the predicted $\mathrm{BMDL}_{10}$ value amounting to $110 \mathrm{mg}$ for a $81.9 \mathrm{~kg}$ person, showing that the PBK modelingbased reverse dosimetry can adequately predicted the in vivo cardiotoxicity of noribogaine. The predicted BMDL 10 value for ibogaine-induced QTc prolongation was $96.9 \mathrm{mg}$ for a $70 \mathrm{~kg}$ person, which is similar to that for noribogaine ( $94.2 \mathrm{mg}$, based on a bodyweight of $70 \mathrm{mg}$ ), indicating a comparable potency of the two compounds in inducing QTc prolongation. 

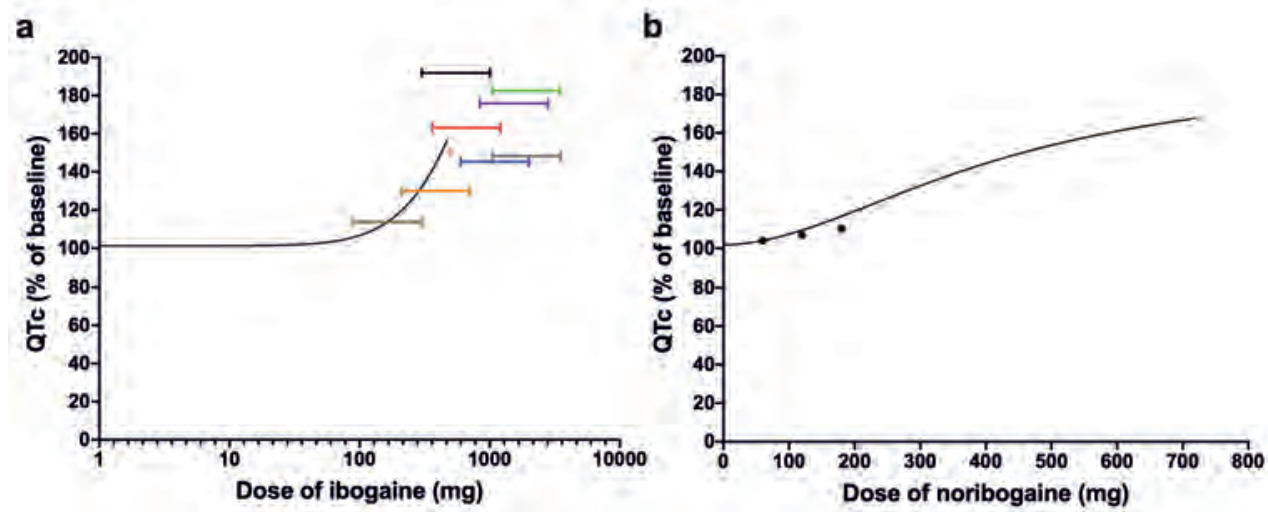

Figure 9 Predicted dose-response curves for cardiotoxicity of (a) ibogaine and (b) noribogaine obtained using PBK modeling-based reverse dosimetry compared to in vivo data derived from literature. The curves represent the predicted dose dependent QTc prolongation. Horizontal bars or the dot in (a) represent the reported data of ibogaine obtained from the following studies: Asua (2013) (grey); Grogan et al. (2019) (black); Henstra et al. (2017) (orange); Hildyard et al. (2015) (green); Hoelen et al. (2009) (pink dot); Meisner et al. (2016) (blue); Pleskovic et al. (2012) (brown); Steinberg and Deyell. (2018) (purple); Vlaanderen et al. (2014) (red). The horizontal bars represent the range of effective doses corrected by multiplying the reported doses with the purity range of internet-purchased ibogaine (15-50\%) (Alper et al. 2012). Dots in (b) represent the in vivo dose-response data for noribogaine reported in Glue et al. (2016).

\section{Discussion and conclusion}

The aim of the present study was to provide an additional proof-of-principle for the potential prediction of in vivo human cardiotoxicity on QTe prolongation by combing an in vitro cardiotoxicity assay, in vitro-derived biokinetic parameters and PBK modeling-based reverse dosimetry as a NAM for human risk and safety assessment. Two herbal alkaloids, ibogaine and noribogaine being promising anti-addiction drugs, were selected as model compounds since their cardiotoxicity is one of the major safety concerns related to their clinical uses, while so far not well studied. In addition, available in vivo kinetic and QTc data available for human subjects exposed to the compounds enable the evaluation of the developed NAM against clinically observed responses.

In the current paper, the electrophysiological cardiotoxicity of ibogaine and noribogaine was assessed using hiPSC-CMs on the MEA platform, which has been used previously for detecting drug-induced QTc prolongation and proarrhythmia (Shi et al., 2020a; Shi et al., 2020b; Satsuka and Kanda, 2020). The results reveal that ibogaine and noribogaine prolonged 
the FPDc in a concentration-dependent manner which could be explained by their inhibitory effects on hERG channels as detected using human mammalian cell lines heterologously expressing hERG channels (Alper et al., 2016; Koenig et al., 2014; Rubi et al., 2017). Our results also indicate that ibogaine was 1.4 -fold more potent than noribogaine in prolonging FPDc, which is in contrast to those hERG inhibition studies reporting a slightly lower (1.3fold) potency of ibogaine compared to noribogaine. The discrepancy might be ascribed to the usage of different cell models. Unlike transfected cell lines containing a single type of ion channels, hiPSC-CMs express the major cardiac ion channels and receptors present in human cardiomyocytes (Karakikes et al., 2015; Kussauer et al., 2019; Ma et al., 2011) and thus the observed results of ibogaine and noribogaine could be the result of multiple ion-channel effects. Rubi et al. (2017) assessed the cardiotoxicity of ibogaine and noribogaine in hiPSC-CMs using one concentration of each compound which prolonged the action potential duration at $90 \%$ repolarization by respectively $14.2 \%$ and $15.5 \%$ indicating comparable potency. Additionally, arrhythmia-type waveforms were observed upon treatment of hiPSC-CMs with ibogaine at high concentrations which is line with case studies where cardiac arrhythmia has been associated with the intake of an overdose ibogaine (Asua, 2013; Paling et al., 2012).

Furthermore, at clinically relevant doses of ibogaine for the treatment of drug addiction (typically 500-1000 mg), the total blood concentration of noribogaine ranged from 0.7 to 4.5 $\mu \mathrm{M}$ (Mash et al., 2008) and unbound blood concentrations ranged from 0.08 to $0.47 \mu \mathrm{M}$ when taking its unbound fraction in plasma and BPr into account. These values cover the unbound in vitro effective concentration $\left(\mathrm{BMCL}_{10}\right)$ of noribogaine being $0.12 \mu \mathrm{M}$, a value corrected for the unbound fraction in the in vitro medium obtained in the current study, indicating that the cardiotoxicity of noribogaine contributes to ibogaine-induced cardiotoxicity and should thus be taken into account in the reverse dosimetry.

Results obtained from in vitro microsomal incubations reveal a high catalytic efficiency for the metabolism of ibogaine to noribogaine, which is in accordance with published data indicating that ibogaine is a compound with high intrinsic clearance with the majority being metabolized to noribogaine (Obach et al., 1998). Based on studies with human liver microsomes Obach et al. (1998) suggested that two enzymes with different activities were involved in the formation of noribogaine, and the apparent $V_{\max }$ and $\mathrm{K}_{\mathrm{m}}$ for the high-affinity enzyme appeared comparable with our data. However, the reported biphasic kinetics were not observed in our incubations. Furthermore, we found that the catalytic efficiency for the conversion of noribogaine to noribogaine glucuronide was quite low, which is consistent with 
the fact that only small amounts of noribogaine glucuronides were reported to be formed following an oral dose of noribogaine (Glue et al., 2015a). The substantially higher catalytic efficiency for conversion of ibogaine to noribogaine than for glucuronidation of noribogaine is also reflected by the kinetic data of the present study, and explains why upon dosing ibogaine, plasma levels of noribogaine exceed those of ibogaine itself. Additionally, by comparing the unbound blood concentration corrected for TEF values of ibogaine and noribogaine, we found that noribogaine is predicted to be a major contributor to the unbound TEQ concentration expressed in ibogaine equivalents. In the light of these findings it can be speculated that noribogaine instead of ibogaine itself plays a dominant role in the in vivo cardiotoxicity upon the oral administration of ibogaine. The discrepancy between the relatively high cardiotoxic potency for ibogaine observed in hiPSC-CM MEA assay and a relatively small contribution to the in vivo cardiotoxicity could be explained by our findings that i) ibogaine can be efficiently and extensively metabolized to noribogaine, ii) that ibogaine highly binds to the plasma protein, resulting a small unbound internal concentration of ibogaine, which is the fraction generally assumed to be responsible for the therapeutic or toxic effect of drugs (Smith et al., 2010), and iii) that clearance of noribogaine to its glucuronide is less efficient and iv) that the protein binding of noribogaine is somewhat less than that of ibogaine resulting in higher unbound concentrations.

The blood $\mathrm{C}_{\max }$ and AUC values of ibogaine and noribogaine predicted by the developed PBK model are comparable with the literature data with differences between the predicted and reported values being less than 2 -fold, indicating an adequate predictive performance of the developed PBK models.

Based on the sensitivity analysis, the influence of metabolic parameters on the predicted $\mathrm{C}_{\max }$ of ibogaine in heart venous blood showed a dose-dependent change. When the oral dose of ibogaine increased to $500 \mathrm{mg}$ the influence of $\mathrm{V}_{\max }$ increased while $\mathrm{K}_{\mathrm{m}}$ was less influential. This may be explained by the fact that at high dose levels metabolism gets saturated, which reduces the influence of the $\mathrm{K}_{\mathrm{m}}$ with metabolic clearance being dependent on $\mathrm{V}_{\max }$. It is also of interest to note that most of the ibogaine related parameters, including metabolic parameters, were not influential to the unbound TEQ concentration expressed in ibogaine equivalents, which however was sensitive to noribogaine related parameters. Furthermore, results show that the unbound TEQ concentration and $\mathrm{C}_{\max }$ of noribogaine in the heart venous blood were affected by these noribogaine related parameters to a similar extent. 
Upon evaluation of the newly defined PBK models the models were used to translate the in vitro concentration response curves obtained in the hiPSC-CM-MEA assay to in vivo dose response curves for cardiotoxicity of ibogaine and noribogaine. To evaluate the predictions of the PBK modeling-based reverse dosimetry NAM, the predicted dose-response curves of ibogaine and noribogaine were compared to available in vivo data. The results obtained show that the predicted dose-response curve for ibogaine is in line with the reported QTc prolongation data especially when the reported doses were corrected for a purity of $15 \%$, which could be considered as a representative and realistic purity of internet-purchased ibogaine as reported by Hoelen et al. (2009). It is important to note the large variation in QTc prolongation data at similar oral doses as observed in some case reports, which may be related to individuals' diverse demographic characteristics and/ or potential QTc prolonging risk factors that were not well-documented in these studies. When applicable, applying exclusion or inclusion criteria to the reported data may better illustrate the dose-dependent effects of ibogaine and thus further improve the accuracy of the evaluation. For noribogaine, the predicted dose-response curve matches well with reported dose-response curves for QTc prolongation with a difference in the $\mathrm{BMDL}_{10}$ values derived from the predicted and reported data being less than 1.5-fold, which further demonstrates that the developed QIVIVE approach can adequately predict the in vivo cardiotoxicity for human.

Based on human experiences the administered dose of ibogaine for treating drug addiction has a wide range and varies from 6 to $30 \mathrm{mg} / \mathrm{kg}$ bw (equal to 420 to $2100 \mathrm{mg}$ for a human of 70 kg) (Alper et al., 1999; Davis et al., 2017; Mash et al., 2018; Noller et al., 2018; Schep et al., 2016). These dose levels are 4-to 21-fold higher than our predicted $\mathrm{BMDL}_{10}$ value (96.9 $\mathrm{mg}$ for a human of $70 \mathrm{~kg}$ ) for the ibogaine dependent induction of QTc prolongation. Since a $\mathrm{BMDL}_{10}$ value generally represents a dose level at which the adverse effect is considered negligible (EFSA, 2017), the prolonged QTc would be expected at doses higher than the predicted BMDL 10 values, which is in line with the observation that QTc prolongations and arrythmia were observed in the subjects administered dose levels of ibogaine above the predicted $\mathrm{BMDL}_{10}$, and that a dose higher than $20 \mathrm{mg} / \mathrm{kg}$ bw ibogaine (equal to $1400 \mathrm{mg}$ for a human of $70 \mathrm{~kg}$ ) is associated with fatalities (Mash et al., 2018). Our predictions also confirm that ECG monitoring is essential for patients receiving ibogaine (Glue et al., 2016). In addition, many studies demonstrate that ibogaine and noribogaine have different neurobiological profiles (Baumann et al., 2001a; Baumann et al., 2001b; Maciulaitis et al., 2008), indicating that noribogaine instead of ibogaine might be more efficient in mediating certain 
pharmacological effects (Mash et al., 2016). Based on our model predictions and reported observations (Glue et al., 2016), a 1.3 to 1.7-fold higher dose of ibogaine is needed to reach the same $\mathrm{C}_{\max }$ of noribogaine compared to the direct intake of noribogaine. Considering a similar in vivo potency observed in the current study for the predicted in vivo induction of the unwanted side effect of QTc prolongation by the two compounds (similar predicted BMDL 10 values), noribogaine would be a safer option.

In the present study we consider that a relatively small contribution of ibogaine itself to the in vivo cardiotoxicity could be due to its low unbound fraction in plasma and its extensive metabolism. However, as ibogaine is a basic compound, the plasma protein binding could be influenced by the level of alphal-acid glycoprotein which has an up to 10-fold variation in human plasma (Smith and Waters, 2019). Furthermore, it has been reported that the internal concentration of ibogaine was up to 43-fold higher in CYP2D6 poor metabolizers compared to extensive metabolizers (Mash et al, 2001; Glue et al., 2015b). Glue et al. (2015b) also reported that the $\mathrm{C}_{\max }$ of ibogaine was 26-fold higher in CYP2D6 extensive metabolizers who took CYP2D6 inhibitors compared to the ones who took placebo. Based on these observations, the cardiotoxicity of ibogaine might become apparent more easily in these sensitive individuals. Moreover, due to the limited information on the metabolism of noribogaine, only the glucuronidation was included in the current model. Other metabolic reactions such as sulfation might be also involved in the clearance of noribogaine (Glue et al., 2016). Considering the relative high potency of noribogaine in in vivo cardiotoxicity, it would be of interest to have a comprehensive metabolic profile for noribogaine defining also potential minor pathways for its clearance.

In conclusion, we demonstrated that integrating in vitro cardiotoxicity data obtained with hiPSC-CMs in the MEA assay, in vitro biokinetic data and PBK modelling can be a promising NAM to predict the in vivo dose-dependent cardiotoxicity of ibogaine and noribogaine in human. The comparison of the predictions obtained to the in vivo data indicated the adequate performance of the developed in vitro in silico approach. Obtained predictions also reveal that a similar in vivo cardiotoxicity potency upon the oral administration of ibogaine and noribogaine while noribogaine might play a substantial role in ibogaine-induced QTc prolongation. Altogether, the present study shows an additional proof of principle for using a NAM consisting of PBK modeling-based reverse dosimetry of hiPSC-CMs MEA assay data for the prediction of human cardiotoxicity, which can be used for cardiac safety evaluation. 


\section{Acknowledgements}

This work was funded by a Grant from the China Scholarship Council (No. 201607720029 to MIAOYING SHI).

\section{Conflicts of interest:}

All authors declare that they have no conflict of interest.

\section{Supplementary data}

For convenience, supplementary materials can be found after reference section 


\section{References}

Alper, K.R. (2001) Ibogaine: A Review. In K. Alper, G. A. Cordell (ed.) The Alkaloids Chemistry and biology 56 (1-38). Amsterdam, The Netherlands: Elsevier. https://doi.org/10.1016/S0099-9598(01)56005-8

Alper, K.R., Bai, R., Liu, N. et al. (2016) hERG blockade by iboga alkaloids. Cardiovascular toxicology 16(1):1422. https://doi.org/10.1007/s12012-015-9311-5

Alper, K.R., Lotsof, H. S., Geerte, M. N. et al. (1999) Treatment of acute opioid withdrawal with ibogaine. American Journal on Addictions 8(3):234-242. https://doi.org/10.1080/105504999305848

Alper, K.R., Stajić, M., Gill, J.R. (2012) Fatalities temporally associated with the ingestion of ibogaine. Journal of forensic sciences 57(2):398-412. https://doi.org/10.1111/j.1556-4029.2011.02008.x

Andersen, M. E., McMullen, P.D., Phillips, M. B. et al. (2019) Developing context appropriate toxicity testing approaches using new alternative methods (NAMs). ALTEX-Alternatives to animal experimentation 36(4):523-534. https://doi.org/10.14573/altex.1906261

Ando, H., Yoshinaga, T., Yamamoto, W. et al. (2017) A new paradigm for drug-induced torsadogenic risk assessment using human iPS cell-derived cardiomyocytes. Journal of pharmacological and toxicological methods 84:111-127. https://doi.org/10.1016/j.vascn.2016.12.003

Asua, I. (2013) Growing menace of ibogaine toxicity. British journal of anaesthesia 111(6):1029-1030. https://doi.org/10.1093/bja/aet396

Barter, Z. E., Bayliss, M. K., Beaune, P. H. et al. (2007) Scaling factors for the extrapolation of in vivo metabolic drug clearance from in vitro data: reaching a consensus on values of human micro-somal protein and hepatocellularity per gram of liver. Current drug metabolism 8(1):33-45. https://doi.org/10.2174/138920007779315053

Barter, Z. E., Tucker, G. T., Rowland-Yeo, K. (2013). Differences in cytochrome p450-mediated pharmacokinetics between chinese and caucasian populations predicted by mechanistic physiologically based pharmacokinetic modelling. Clinical pharmacokinetics 52: 1085-1100. https://doi.org/10.1007/s40262-013-0089-y

Baumann, M. H., Pablo, J., Ali, S. F. et al. (2001a) Comparative neuropharmacology of ibogaine and its Odesmethyl metabolite, noribogaine. In K. Alper, G. A. Cordell (ed.) The Alkaloids Chemistry and biology 56 (79-113). Amsterdam, The Netherlands: Elsevier.https://doi.org/10.1016/S0099-9598(01)56009-5

Baumann, M. H., Rothman, R. B., Pablo, J. P., Mash, D. C. (2001b) In vivo neurobiological effects of ibogaine and its O-desmethyl metabolite, 12-hydroxyibogamine (noribogaine), in rats. Journal of Pharmacology and Experimental Therapeutics 297(2):531-539. https://pubmed.ncbi.nlm.nih.gov/11303040/

Bentz, J., O'Connor, M. P., Bednarczyk, D. et al. (2013) Variability in P-glycoprotein inhibitory potency (IC50) using various in vitro experimental systems: implications for universal digoxin drug-drug interaction risk assessment decision criteria. Drug Metabolism and Disposition 41(7):1347-1366. https://doi.org/10.1124/dmd.112.050500

Berezhkovskiy, L. M. (2004) Volume of distribution at steady state for a linear pharmacokinetic system with peripheral elimination. Journal of pharmaceutical sciences 93(6):1628-1640. https://doi.org/10.1002/jps.20073

Bos, P. M., Geraets, L., de Wit-Bos, L. et al. (2020) Towards an animal-free human health assessment: Starting from the current regulatory needs. ALTEX-Alternatives to animal experimentation 37(3):395-408. https://doi.org/10.14573/altex.1912041 
Brown, R. P., Delp, M. D., Lindstedt, S. L. et al. (1997) Physiological parameter values for physiologically based pharmacokinetic models. Toxicology and industrial health 13(4):407-484. https://doi.org/10.1177/074823379701300401

Davis, A. K., Barsuglia, J. P., Windham-Herman, A. M. et al. (2017) Subjective effectiveness of ibogaine treatment for problematic opioid consumption: short-and long-term outcomes and current psychological functioning. Journal of psychedelic studies 1(2):65-73. https://doi.org/10.1556/2054.01.2017.009

European Food Safety Authority (EFSA) (2017) Update: use of the benchmark dose approach in risk assessment. https://doi.org/10.2903/j.efsa.2017.4658

Fisher, M. B., Campanale, K., Ackermann, B. L. et al. (2000) In vitro glucuronidation using human liver microsomes and the pore-forming peptide alamethicin. Drug metabolism and disposition 28(5):560-566. https://pubmed.ncbi.nlm.nih.gov/10772635/

Florian, J., Garnett, C., Nallani, S. et al. (2012) A modeling and simulation approach to characterize methadone QT prolongation using pooled data from five clinical trials in MMT patients. Clinical Pharmacology \& Therapeutics 91:666-672. https://doi.org/10.1038/clpt.2011.273

Foster, D. J., Somogyi, A. A., Dyer, K. R. et al. (2000) Steady-state pharmacokinetics of (R)-and (S)-methadone in methadone maintenance patients. British journal of clinical pharmacology 50(5):427-440. https://doi.org/10.1046/j.1365-2125.2000.00272.x

Gilbert-Sandoval, I., Wesseling, S., Rietjens, I. M. C. M. (2020) Predicting the Acute Liver Toxicity of Aflatoxin B1 in Rats and Humans by an In Vitro-In Silico Testing Strategy. Molecular nutrition \& food research 64(13):2000063. https://doi.org/10.1002/mnfr.202000063

Glue, P., Cape, G., Tunnicliff, D. et al. (2016) Ascending single-dose, double-blind, placebo-controlled safety study of noribogaine in opioid-dependent patients. Clinical pharmacology in drug development 5(6):460468. https://doi.org/10.1002/cpdd.254

Glue, P., Lockhart, M., Lam, F. et al. (2015a) Ascending-dose study of noribogaine in healthy volunteers: Pharmacokinetics, pharmacodynamics, safety, and tolerability. The Journal of Clinical Pharmacology 55(2):189-194. https://doi.org/10.1002/jcph.404

Glue, P., Winter, H., Garbe, K. et al. (2015b) Influence of CYP2D6 activity on the pharmacokinetics and pharmacodynamics of a single $20 \mathrm{mg}$ dose of ibogaine in healthy volunteers. The Journal of Clinical Pharmacology 55(6):680-687. https://doi.org/10.1002/jcph.471

Goutarel, R., Gollnhofer, O., Sillans, R. (1993) Pharmacodynamics and therapeutic applications of iboga and ibogaine. Psychedelic Monographs and Essays 6 (71-111) https://ibogainedossier.com/bwitil.html

Graham, H., Walker, M., Jones, O. et al. (2012) Comparison of in-vivo and in-silico methods used for prediction of tissue: plasma partition coefficients in rat. Journal of Pharmacy and Pharmacology 64(3):383-396. https://doi.org/10.1111/j.2042-7158.2011.01429.x

Grogan, J., Gerona, R., Snow, J. W., Kao, L. (2019) Ibogaine Consumption With Seizure-Like Episodes, QTcProlongation, and Captured Cardiac Dysrhythmias. The Journal of emergency medicine 57(4):e99-e104. https://doi.org/10.1016/j.jemermed.2019.06.05

Haddad, S., Nong, A. (2020) Physiologically based pharmacokinetic model: excretion via urine, feces, and breath Physiologically Based Pharmacokinetic (PBPK) Modeling. In J. Fisher, J. Gearhart, Z. Lin. (ed.) Physiologically Based Pharmacokinetic (PBPK) Modeling: Methods and Applications in Toxicology and 
Risk Assessment (175-209). Amsterdam, The Netherlands: Elsevier. https://doi.org/10.1016/B978-0-12818596-4.00008-4

Henstra, M., Wong, L., Chahbouni, A., Swart, N. et al. (2017) Toxicokinetics of ibogaine and noribogaine in a patient with prolonged multiple cardiac arrhythmias after ingestion of internet purchased ibogaine. Clinical Toxicology 55(6):600-602. https://doi.org/10.1080/15563650.2017.1287372

Hildyard, C., Macklin, P., Prendergast, B., Bashir, Y. (2016) A case of QT prolongation and torsades de pointes caused by ibogaine toxicity. Journal of Emergency Medicine 50(2):e83-e87. https://doi.org/10.1016/j.jemermed.2015.06.05

Hoelen, D. W., Spiering, W., Valk, G. D. (2009) Long-QT syndrome induced by the antiaddiction drug ibogaine. New England journal of medicine 360(3):308-309. https://doi.org/10.1056/NEJMc0804248

Hubatsch, I., Ragnarsson, E. G., Artursson, P. (2007) Determination of drug permeability and prediction of drug absorption in Caco-2 monolayers. Nature protocols 2(9):2111. https://doi.org/10.1038/nprot.2007.303

Interagency Coordinating Committee on the Validation of Alternative Methods (ICCVAM) (2018). A Strategic Roadmap for Establishing New Approaches to Evaluate the Safety of Chemicals and Medical Products in the United States. https://dx.doi.org/10.22427/NTP-ICCVAM-ROADMAP2018.

Jeffcoat, A. R., Cook, C. E., Hill, J. M. et al. (2013). Disposition of [3H] ibogaine in the rat. Problems of Drug Dependence: Proceedings of the 55th Annual Scientific Meeting, the College on Problems of Drug Dependence, Inc., p 141: 309.

Karakikes, I., Ameen, M., Termglinchan, V., Wu, J. C. (2015) Human induced pluripotent stem cell-derived cardiomyocytes: insights into molecular, cellular, and functional phenotypes. Circulation research 117(1):80-88. https://doi.org/10.1161/CIRCRESAHA.117.305365

Kitaguchi, T., Moriyama, Y., Taniguchi, T. et al. (2017) CSAHi study: detection of drug-induced ion channel/receptor responses, QT prolongation, and arrhythmia using multi-electrode arrays in combination with human induced pluripotent stem cell-derived cardiomyocytes. Journal of pharmacological and toxicological methods 85:73-81. https://doi.org/10.1016/j.vascn.2017.02.001

Koenig, X., Kovar, M., Boehm, S. et al. (2014) Anti-addiction drug ibogaine inhibits hERG channels: a cardiac arrhythmia risk. Addiction biology 19(2):237-239. https://doi.org/10.1111/j.1369-1600.2012.00447.x

Kontrimavičiūtè, V., Mathieu, O., Mathieu-Daudé, J.C. et al. (2006) Distribution of ibogaine and noribogaine in a man following a poisoning involving root bark of the Tabernanthe iboga shrub. Journal of analytical toxicology 30(7):434-440. https://doi.org/10.1093/jat/30.7.434

Kussauer, S., David, R., Lemcke, H. (2019) hiPSCs derived cardiac cells for drug and toxicity screening and disease modeling: what micro-electrode-array analyses can tell us. Cells 8(11):1331. https://doi.org/10.3390/cells8111331

Litjens, R. P., Brunt, T. M. (2016) How toxic is ibogaine? Clinical Toxicology 54(4):297-302. https://doi.org/10.3109/15563650.2016.1138226

Louisse, J., de Jong, E., van de Sandt, J. J. et al. (2010) The use of in vitro toxicity data and physiologically based kinetic modeling to predict dose-response curves for in vivo developmental toxicity of glycol ethers in rat and man. Toxicological Sciences 118(2):470-484. https://doi.org/10.1093/toxsci/kfq270

Lozoya-Agullo, I., González-Álvarez, I., González-Álvarez, M. et al. (2015) In situ perfusion model in rat colon for drug absorption studies: comparison with small intestine and Caco-2 cell model. Journal of pharmaceutical sciences 104(9):3136-3145. https://doi.org/10.1002/jps.24447 
Lüpfert, C., Reichel, A. (2005) Development and application of physiologically based pharmacokinetic-modeling tools to support drug discovery. Chemistry \& biodiversity 2(11):1462-1486. https://doi.org/10.1002/cbdv.200590119

Ma, J., Guo, L., Fiene, S. J. et al. (2011) High purity human-induced pluripotent stem cell-derived cardiomyocytes: electrophysiological properties of action potentials and ionic currents. American Journal of PhysiologyHeart and Circulatory Physiology 301(5):H2006-H2017. https://doi.org/10.1152/ajpheart.00694.2011

Maciulaitis, R., Kontrimaviciute, V., Bressolle, F., Briedis, V. (2008) Ibogaine, an anti-addictive drug: pharmacology and time to go further in development. A narrative review. Human and Experimental Toxicology 27(3):181. https://doi.org/10.1177/0960327107087802

Martin, R. L., McDermott, J. S., Salmen, H. J. et al. (2004) The utility of hERG and repolarization assays in evaluating delayed cardiac repolarization: influence of multi-channel block. Journal of cardiovascular pharmacology 43(3):369-379. https://doi.org/10.1097/00005344-200403000-00007

Mash, D. C., Kovera, C. A., Pablo, J. et al. (2001). Ibogaine in the treatment of heroin withdrawal. The Alkaloids Chemistry and biology 56 (155-171). Amsterdam, The Netherlands: Elsevier. https://doi.org/10.1016/S0099-9598(01)56012-5

Mash, D. C., Ameer, B., Prou, D. et al. (2016) Oral noribogaine shows high brain uptake and anti-withdrawal effects not associated with place preference in rodents. Journal of Psychopharmacology 30(7):688-697. https://doi.org/10.1177/0269881116641331

Mash, D. C., Duque, L., Page, B., Allen-Ferdinand. K. (2018) Ibogaine detoxification transitions opioid and cocaine abusers between dependence and abstinence: clinical observations and treatment outcomes. Frontiers in pharmacology 9:529. https://doi.org/10.3389/fphar.2018.00529

Meisner, J. A., Wilcox, S. R., Richards, J. B. (2016) Ibogaine-associated cardiac arrest and death: case report and review of the literature. Therapeutic advances in psychopharmacology 6(2):95-98. https://doi.org/10.1177/2045125315626073

Mujtaba, S., Romero, J., Taub, C. C. (2013) Methadone, QTc prolongation and torsades de pointes: current concepts, management and a hidden twist in the tale? Journal of Cardiovascular Disease Research 4:229-235. https://doi.org/10.1016/j.jcdr.2013.10.001

Ning, J., Louisse, J., Spenkelink, B. et al. (2017) Study on inter-ethnic human differences in bioactivation and detoxification of estragole using physiologically based kinetic modeling. Archives of toxicology 91(9):3093-3108. https://doi.org/10.1007/s00204-017-1941-x

Noller, G. E, Frampton, C. M., Yazar-Klosinski, B. (2018) Ibogaine treatment outcomes for opioid dependence from a twelve-month follow-up observational study. The American journal of drug and alcohol abuse 44(1):37-46. https://doi.org/10.1080/00952990.2017.1310218

Nozaki, Y., Honda, Y., Watanabe, H. et al. (2017) CSAHi study-2: validation of multi-electrode array systems (MEA60/2100) for prediction of drug-induced proarrhythmia using human iPS cell-derived cardiomyocytes: assessment of reference compounds and comparison with non-clinical studies and clinical information. Regulatory Toxicology and Pharmacology 88:238-251. https://doi.org/10.1016/j.yrtph.2017.06.006

O'Connell, C. W., Gerona, R. R, Friesen, M. W, Ly, B. T. (2015) Internet-purchased ibogaine toxicity confirmed with serum, urine, and product content levels. The American journal of emergency medicine 33(7):985. e5-985. e6. https://doi.org/10.1016/j.ajem.2014.12.023 
Obach, R. S, Pablo, J., Mash, D. C. (1998) Cytochrome P4502D6 catalyzes the O-demethylation of the psychoactive alkaloid ibogaine to 12-hydroxyibogamine. Drug metabolism and disposition 26(8):764768. https://pubmed.ncbi.nlm.nih.gov/9698290/

Ovics, P., Regev, D., Baskin, P. et al. (2020) Drug Development and the Use of Induced Pluripotent Stem CellDerived Cardiomyocytes for Disease Modeling and Drug Toxicity Screening. International Journal of Molecular Sciences 21(19):7320. https://doi.org/10.3390/ijms21197320

Paling, F., Andrews, L., Valk, G., Blom, H. (2012) Life-threatening complications of ibogaine: three case reports. The Netherlands Journal of Medicine 70(9):422-4. https://pubmed.ncbi.nlm.nih.gov/23123541/

Pang, L., Sager, P., Yang, X. et al. (2019) Workshop report: FDA workshop on improving cardiotoxicity assessment with human-relevant platforms. Circulation research 125(9):855-867. https://doi.org/10.1161/CIRCRESAHA.119.315378

Patterson, E. A., Whelan, M. P., Worth, A. P. (2020) The role of validation in establishing the scientific credibility of predictive toxicology approaches intended for regulatory application. Computational Toxicology:100144. https://doi.org/10.1016/j.comtox.2020.100144

Pleskovic, A., Gorjup, V., Brvar, M., Kozelj, G. (2012) Ibogaine-associated ventricular tachyarrhythmias. Clinical Toxicology 50 (2): 157-157. https://doi.org/10.3109/15563650.2011.647031

Punt, A., Bouwmeester, H., Blaauboer, B. J. et al. (2020) New approach methodologies (NAMs) for humanrelevant biokinetics predictions: Meeting the paradigm shift in toxicology towards an animal-free chemical risk assessment. ALTEX-Alternatives to animal experimentation 37(4):607-622. https://doi.org/10.14573/altex.2003242

Rietjens, I. M. C. M., Louisse, J., Punt, A. (2011) Tutorial on physiologically based kinetic modeling in molecular nutrition and food research. Molecular nutrition \& food research 55(6):941-956. https://doi.org/10.1002/mnfr.201000655

Rodgers, T., Rowland, M. (2006) Physiologically based pharmacokinetic modelling 2: predicting the tissue distribution of acids, very weak bases, neutrals and zwitterions. Journal of pharmaceutical sciences 95(6):1238-1257. https://doi.org/10.1002/jps.20502

Rohatgi, A. (2020). WebPlotDigitizer (Version 4.4) [Computer software]. Retrieved from https://apps.automeris.io/wpd/

Rubi, L., Eckert, D., Boehm, S. et al. (2017) Anti-addiction drug ibogaine prolongs the action potential in human induced pluripotent stem cell-derived cardiomyocytes. Cardiovascular toxicology 17(2):215-218. https://doi.org/10.1007/s12012-016-9366-y

Sala, L., Ward-van Oostwaard, D., Tertoolen, L. G. et al. (2017) Electrophysiological analysis of human pluripotent stem cell-derived cardiomyocytes (hPSC-CMs) using multi-electrode arrays (MEAs). JoVE (Journal of Visualized Experiments)(123):e55587. https://doi.org/10.3791/55587

Satsuka, A., Kanda, Y. (2020) Cardiotoxicity Assessment of Drugs Using Human iPS Cell-Derived Cardiomyocytes: Toward Proarrhythmic Risk and Cardio-Oncology. Current pharmaceutical biotechnology 21(9):765-772. https://doi.org/10.2174/1389201020666190628143345

Schep, L. J., Slaughter, R., Galea, S., Newcombe, D. (2016) Ibogaine for treating drug dependence. What is a safe dose? Drug and alcohol dependence 166:1-5. https://doi.org/10.1016/j.drugalcdep.2016.07.005

Shi, M., Bouwmeester, H., Rietjens, I. M. C. M., Strikwold, M. (2020a) Integrating in vitro data and physiologically based kinetic modeling-facilitated reverse dosimetry to predict human cardiotoxicity of methadone. Archives of toxicology 94(8):2809-2827. https://doi.org/10.1007/s00204-020-02766-7 
Shi, M., Tien, N. T., de Haan, L. et al. (2020b). Evaluation of in vitro models of stem cell-derived cardiomyocytes to screen for potential cardiotoxicity of chemicals. Toxicology in Vitro: 104891. https://doi.org/10.1016/j.tiv.2020.104891

Skolnik, S., Lin, X., Wang, J. et al. (2010) Towards prediction of in vivo intestinal absorption using a 96-well Caco-2 assay. Journal of pharmaceutical sciences 99(7):3246-3265. https://doi.org/10.1002/jps.22080

Smith, D. A., Di, L., Kerns, E. H. (2010) The effect of plasma protein binding on in vivo efficacy: misconceptions in drug discovery. Nature reviews Drug discovery 9(12):929-939. https://doi.org/10.1038/nrd3287

Smith, S. A., Waters, N. J. (2019) Pharmacokinetic and pharmacodynamic considerations for drugs binding to alpha-1-acid glycoprotein. Pharmaceutical research 36(2):1-19. https://doi.org/10.1007/s11095-0182551-x

Steinberg, C., Deyell, M. W. (2018) Cardiac arrest after ibogaine intoxication. Journal of arrhythmia 34(4):455457. https://doi.org/10.1002/joa3.12061

Strikwold, M., Spenkelink, B., de Haan, L. et al. (2017) Integrating in vitro data and physiologically based kinetic (PBK) modelling to assess the in vivo potential developmental toxicity of a series of phenols. Archives of toxicology 91(5):2119-2133. https://doi.org/10.1007/s00204-016-1881-x

Taboureau, O., El M'Selmi, W., Audouze, K. (2020) Integrative systems toxicology to predict human biological systems affected by exposure to environmental chemicals. Toxicology and Applied Pharmacology 405:115210. https://doi.org/10.1016/j.taap.2020.115210

The International Council for Harmonisation of Technical Requirements for Pharmaceuticals for Human Use (ICH) (2005) E14: the clinical evaluation of QT/QTc interval prolongation and proarrhythmic potential for non-antiarrhythmic drugs. https:// database.ich.org/sites/default/files/E14_Guideline.pdf. Accessed 20 Jan 2021

Utsey, K., Gastonguay, M.S., Russell, S. et al. (2020) Quantification of the Impact of Partition Coefficient Prediction Methods on Physiologically Based Pharmacokinetic Model Output Using a Standardized Tissue Composition. Drug Metabolism and Disposition 48(10):903-916. https://doi.org/10.1124/dmd.120.090498

van Liempd, S., Morrison, D., Sysmans, L. et al. (2011) Development and validation of a higher-throughput equilibrium dialysis assay for plasma protein binding. JALA: Journal of the Association for Laboratory Automation 16(1):56-67. https://doi.org/10.1016/j.jala.2010.06.002

Vandenberk B, Vandael E, Robyns T, et al. (2016) Which QT correction formulae to use for QT monitoring? Journal of the American Heart Association 5(6):e003264. https://doi.org/10.1161/JAHA.116.003264

Vlaanderen, L., Martial, L., Franssen, E. et al. (2014) Cardiac arrest after ibogaine ingestion. Clinical Toxicology 52(6):642-643. https://doi.org/10.3109/15563650.2014.927477

Waters, N. J., Jones, R., Williams, G., Sohal, B. (2008) Validation of a rapid equilibrium dialysis approach for the measurement of plasma protein binding. Journal of pharmaceutical sciences 97(10):4586-4595. https://doi.org/10.1002/jps.21317

Wedam, E. F., Bigelow, G. E., Johnson, R. E. et al. (2007) QT-interval effects of methadone, levomethadyl, and buprenorphine in a randomized trial. Archives of Internal Medicine 167:2469-2475. https://doi.org/10.1001/archinte.167.22.2469

Wolff, K., Rostami-Hodjegan, A., Hay, A. et al. (2000) Population-based pharmacokinetic approach for methadone monitoring of opiate addicts: potential clinical utility. Addiction 95(12):1771-1783. https://doi.org/10.1046/j.1360-0443.2000.951217717.x 
Zhao, S., Kamelia, L., Boonpawa, R. et al. (2019) Physiologically based kinetic modeling-facilitated reverse dosimetry to predict in vivo red blood cell acetylcholinesterase inhibition following exposure to chlorpyrifos in the Caucasian and Chinese population. Toxicological sciences 171(1):69-83. https://doi.org/10.1093/toxsci/kfz134

Wageningen Food Safety Research (WFSR) (2020). QIVIVE tools-Partition coefficients. [Online]. Available at: https://wfsr.shinyapps.io/wfsrqivivetools/ (Accessed: 15th December 2020).

Zwartsen, A., de Korte, T., Nacken, P. et al. (2019) Cardiotoxicity screening of illicit drugs and new psychoactive substances (NPS) in human iPSC-derived cardiomyocytes using microelectrode array (MEA) recordings. Journal of molecular and cellular cardiology 136:102-112. https://doi.org/10.1016/j.yjmcc.2019.09.007 


\section{Supplementary materials 1}
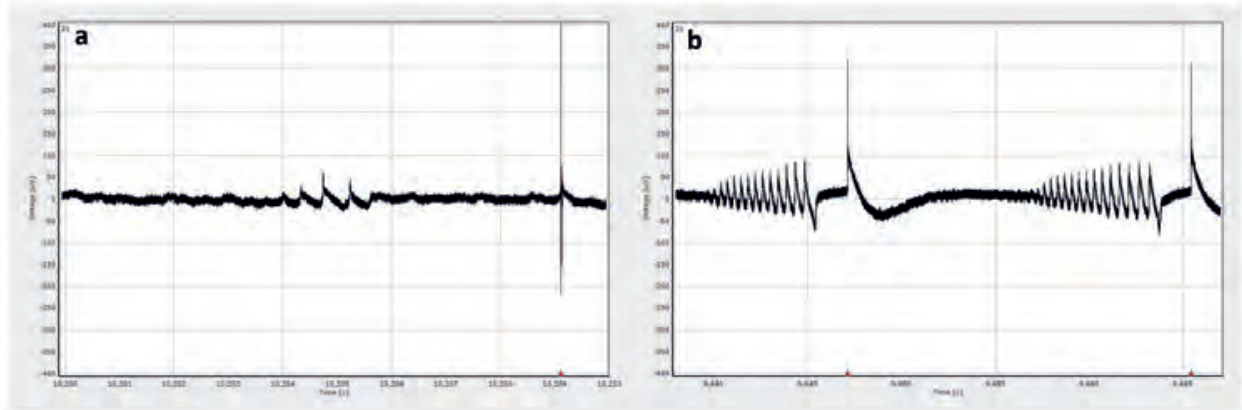

Figure S1 Irregular waveforms of field potential observed in human induced pluripotent stem cellderived cardiomyocytes using the multiple-electrode array. a, arrhythmia-type waveform induced by 1 $\mu \mathrm{M}$ ibogaine. $\mathbf{b}$, arrhythmia-type waveform induced by $3 \mu \mathrm{M}$ noribogaine. Waveforms present in a and $\mathrm{b}$ were not used for defining the in vitro concentration-response curves for FPDc effects.

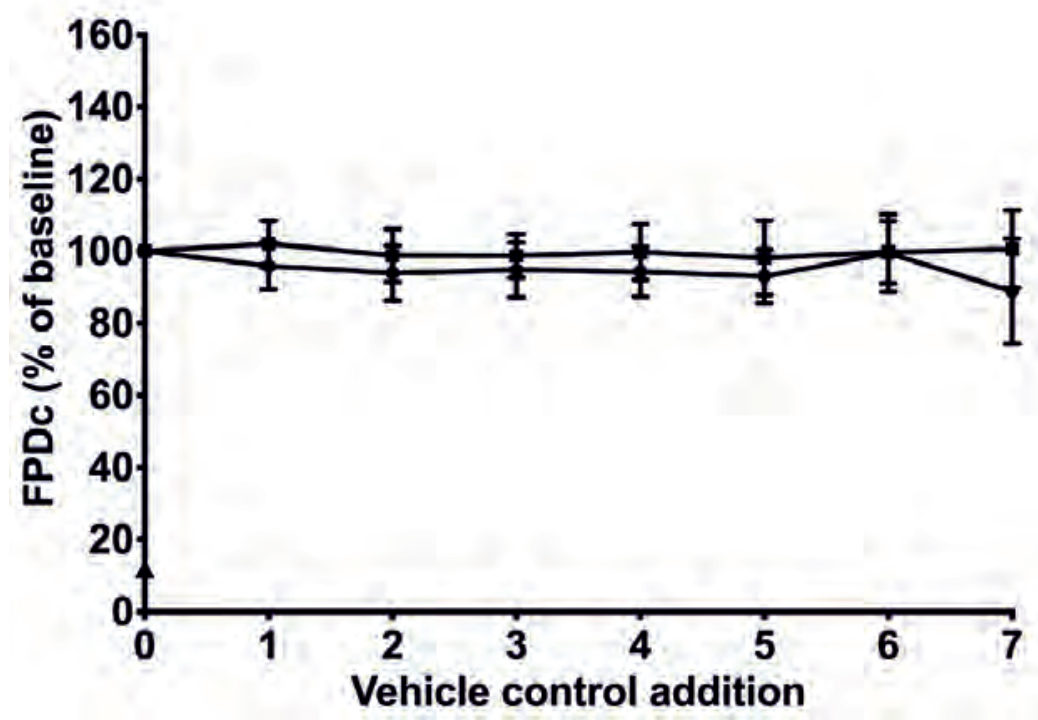

Figure S2 Effects of repeated addition of $0.05 \%(\mathrm{v} / \mathrm{v})$ acetonitrile (squares) and $0.1 \%(\mathrm{v} / \mathrm{v})$ DMSO (circles) on the FPDc relative to baseline conditions in the vehicle control well set at $100 \%$. Vehicle control addition 0 on the $\mathrm{X}$ axis represents the response of the baseline control set at 100\%. 1-7 represent the $1^{\text {st }}$ to $7^{\text {th }}$ addition of vehicle controls corresponding to the $1^{\text {st }}$ to $7^{\text {th }}$ addition. Each data point represents the mean $\pm \mathrm{SD}$ of three independent experiments. 

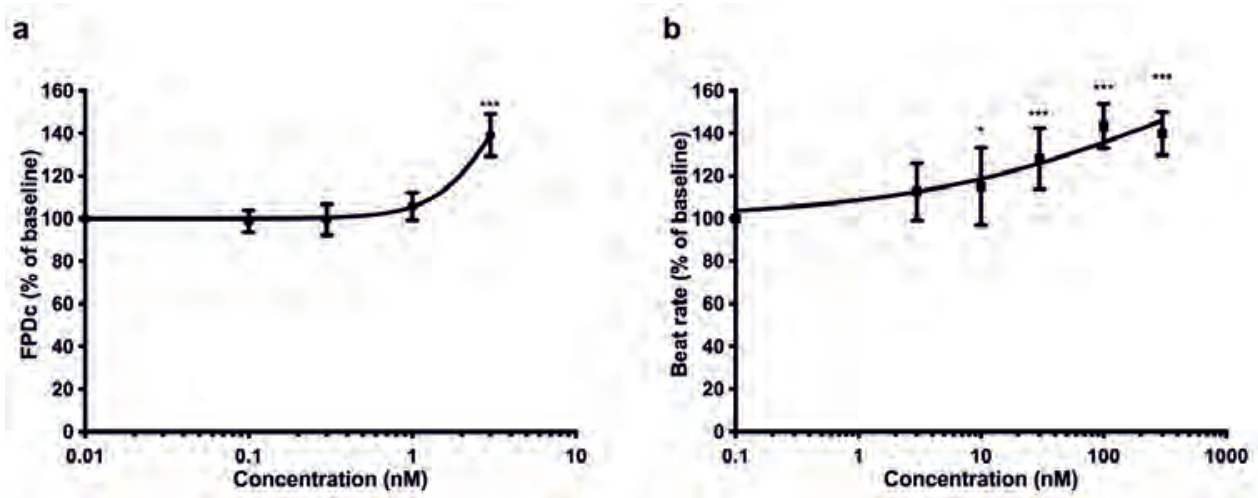

Figure S3 Concentration-response curves for cardiotoxicity in hiPSC-CMs of the reference compounds (a) dofetilide and (b) isoproterenol. The response of the baseline at $0.1 \%(\mathrm{v} / \mathrm{v})$ DMSO was set at 100\%. Data represent the mean of results obtained from three independent experiments each containing six well replicates. Each data point represents the mean \pm SD. Statistically significant changes in response compared to the solvent control are marked with ${ }^{*}$ with $p<0.05:{ }^{*}, p<0.01:{ }^{* *}$ and $p<0.001:{ }^{* * *}$.

Table S1 Summary of case reports of QT prolongation upon oral administration of ibogaine

\begin{tabular}{|c|c|c|c|c|c|c|}
\hline Reference $^{\mathrm{a}}$ & $\begin{array}{l}\text { Reason for ibogaine } \\
\text { use }\end{array}$ & Sex & $\begin{array}{l}\text { Dose } \\
(\mathrm{mg} / \text { day })^{\mathrm{a}}\end{array}$ & $\begin{array}{l}\text { Baseline } \\
\text { QTc (ms) } \\
\text { b }\end{array}$ & $\begin{array}{l}\text { Post } \\
\text { QTc } \\
(\mathrm{ms})\end{array}$ & $\begin{array}{l}\text { QTc (\% to } \\
\text { baseline) }\end{array}$ \\
\hline Asua (2013) & Heroin addiction & Male & 7000 & 405 & 600 & 148.1 \\
\hline $\begin{array}{l}\text { Grogan et al. } \\
(2019)\end{array}$ & $\begin{array}{l}\text { Cocaine and heroin } \\
\text { addiction }\end{array}$ & Female & 2000 & 411 & 788 & 191.7 \\
\hline $\begin{array}{l}\text { Henstra et al. } \\
(2017)\end{array}$ & Heroin addiction & Female & 1400 & 411 & 647 & 130.2 \\
\hline $\begin{array}{l}\text { Hildyard et al. } \\
(2015)\end{array}$ & Heroin addiction & Male & 7000 & 405 & 730 & 182.5 \\
\hline $\begin{array}{l}\text { Hoelen et al. } \\
(2009)\end{array}$ & Alcohol addiction & Female & $500^{\mathrm{c}}$ & 411 & 616 & 149.9 \\
\hline $\begin{array}{l}\text { Meisner et al. } \\
(2016)\end{array}$ & Heroin addiction & Male & 4000 & 405 & 588 & 145.2 \\
\hline $\begin{array}{l}\text { Pleskovic et al. } \\
(2012)\end{array}$ & Not reported & Male & 600 & 405 & 460 & 113.6 \\
\hline $\begin{array}{l}\text { Steinberg et al. } \\
(2018)\end{array}$ & Opioid addiction & Male & 5600 & 405 & 714 & 176.3 \\
\hline $\begin{array}{l}\text { Vlaanderen et } \\
\text { al. (2014) }\end{array}$ & Not reported & Male & 2400 & 405 & 663 & 163.7 \\
\hline
\end{tabular}

a internet-purchased ibogaine with unknown purity. ${ }^{\mathrm{b}}$ Baseline was assumed to be $405 \mathrm{~ms}$ and $411 \mathrm{~ms}$ for male and female, respectively, given that no baseline information was reported (Wedam et al., 2007). ${ }^{\mathrm{c}}$ a dose of $3500 \mathrm{mg}$ ibogaine was corrected for the reported purity of $15 \%$. 


\section{Supplementary materials 2}

;PBK model code human model

; physiological parameters

; Tissue volumes ( $\mathrm{L}$ or $\mathrm{Kg}$ )
$\mathrm{BW}=70$
; body weight human in $\mathrm{kg}$

; All fractions are taken from Brown et al. (1997)

VLc $=0.0257$
VFc $=0.2142$
VLuc $=0.0076$
VAc $=0.0198$
VVc $=0.0593$
$V K c=0.004$
$V H c=0.0047$
VRc $=0.037$
$V S c=0.58$

; fraction of liver tissue

; fraction of fat tissue

; fraction of lung tissue

; fraction of arterial blood: $0.074 * 1 / 4$

; fraction of venous blood: $0.074 * 3 / 4$

; fraction of kidney tissue

; fraction of heart tissue

; fraction of rapidly perfused tissue ; fraction of blood flow to slowly perfused tissue ; total of fractions $=0.9527$

$\mathrm{VL}=\mathrm{VLc} * \mathrm{BW}$
$\mathrm{VF}=\mathrm{VFc} * \mathrm{BW}$
$\mathrm{VLu}=\mathrm{VLuc} * \mathrm{BW}$
$\mathrm{VK}=\mathrm{VKc} * \mathrm{BW}$
$\mathrm{VH}=\mathrm{VHc} * \mathrm{BW}$
$\mathrm{VR}=\mathrm{VRc} * \mathrm{BW}$
$\mathrm{VS}=\mathrm{VSc} * \mathrm{BW}$
$\mathrm{VA}=\mathrm{VAc} * \mathrm{BW}$
$\mathrm{VV}=\mathrm{VVc} * \mathrm{BW}$
$;------------9)$
$; \mathrm{Blood}$ flow rates $(\mathrm{L} / \mathrm{h})$
$\mathrm{QC}=15 * \mathrm{BW} 0.74$
$\mathrm{QLc}=0.227$
$\mathrm{QFc}=0.052$
$\mathrm{QKc}=0.175$
$\mathrm{QHc}=0.04$
$\mathrm{QSc}=0.291$
$\mathrm{QRc}=0.215$

; volume of liver

; volume of fat ; volume of lung

; volume of kidney

; volume of heart

; volume of rapidly perfused tissue

; volume of slowly perfused tissue

; volume of arterial blood

; volume of venous blood

; all fractions are taken from Brown et al. (1997)

$$
\begin{aligned}
& \mathrm{QL}=\mathrm{QLc} * \mathrm{QC} \\
& \mathrm{QF}=\mathrm{QFc} * \mathrm{QC} \\
& \mathrm{QK}=\mathrm{QKc} * \mathrm{QC} \\
& \mathrm{QH}=\mathrm{QHc} * \mathrm{QC} \\
& \mathrm{QR}=\mathrm{QRc} * \mathrm{QC} \\
& \mathrm{QS}=\mathrm{QSc} * \mathrm{QC}
\end{aligned}
$$

; $\mathrm{QC}=15 * \mathrm{BW}^{\wedge} 0.74$ (Brown et al., 1997)

; fraction of blood flow to liver

; fraction of blood flow to fat

; fraction of blood flow to kidney

\begin{tabular}{|c|c|}
\hline ; Partition coefficients & \\
\hline PFibo $=0.18$ & ; fat/blood partition coefficient ibogaine \\
\hline PSibo $=2.73$ & ; slowly perfused tissues/blood partition coefficient ibogaine \\
\hline PHibo $=0.7$ & ; heart/blood partition coefficient ibogaine \\
\hline PKibo $=1.02$ & ; kidney/blood partition coefficient ibogaine \\
\hline PLibo $=1.62$ & ; liver/blood partition coefficient ibogaine \\
\hline PRibo $=1.62$ & ; rapidly perfused tissues/blood partition coefficient ibogaine \\
\hline PLuibo $=0.32$ & ; lung/blood partition coefficient ibogaine \\
\hline PFnor $=1.38$ & ; fat/blood partition coefficient noribogaine \\
\hline
\end{tabular}

; fraction of blood flow to heart

; fraction of blood flow to slowly perfused tissue

; fraction of blood flow to rapidly perfused tissue

$$
\text { ; total of fractions }=1
$$


PSnor $=2.33$

PHnor $=7.6$

PKnor $=16.9$

PLnor $=15.3$

PRnor $=15.3$

PLunor $=13.1$
; slowly perfused tissues/blood partition coefficient noribogaine ; heart/blood partition coefficient noribogaine

; kidney/blood partition coefficient noribogaine

; liver/blood partition coefficient noribogaine

; rapidly perfused tissues/blood partition coefficient noribogaine

; lung/blood partition coefficient noribogaine

\footnotetext{
; Biochemical parameters

$;===========================================$
$;$ Linear uptake rate $(/ \mathrm{h}) \quad ;$ calculated based on $P_{\text {app }}$ values obtained from the current study using methadone as a reference compound.

kaibo $=0.79$

kanor $=1.23$

; Fraction absorbed

Faibo $=1$

Fanor $=1$

; Biliary excretion

kbibo $=0.575$

; the kb of noribogaine was assumed to be same for ibogaine

kbnor $=0.575$

; biliary excretion rate constant $(/ \mathrm{h})$ of noribogaine was obtained by

fitting CVBnor to reported in vivo data (Glue et al., 2016; Glue et al., 2015a; Glue et al., 2015b).
}

; Metabolism of ibogaine in the liver

; Scaling factors;

$\mathrm{MPL}=32$

2007)

$\mathrm{L}=\mathrm{VLc} * 1000$

; liver microsomal protein yield (mg/gram liver) (Barter et al.,

; liver $=25.7($ gram $/ \mathrm{kg} \mathrm{BW})$

; Metabolites of ibogaine, unscaled maximum rate of metabolism (nmol/mg protein $/ \mathrm{min}$ )

Vmaxc1 $=0.17 \quad$; obtained from in vitro microsomal incubation in the current study.

; Metabolites of ibogaine, scaled maximum rate of metabolism $(\mu \mathrm{mol} / \mathrm{h})$

$\mathrm{Vmax} 1=\mathrm{Vmaxc} 1 / 1000 * 60 * \mathrm{MPL} * \mathrm{~L} * \mathrm{BW}$

; Metabolites of ibogaine, affinity constants $(\mu \mathrm{mol} / \mathrm{L})$

$\mathrm{Km} 1=0.63 \quad$; obtained from in vitro microsomal incubation in the current study.

; metabolism of noribogaine in the liver

; Metabolites of noribogaine, unscaled maximum rate of metabolism ( $\mathrm{nmol} / \mathrm{mg}$ protein $/ \mathrm{min}$ )

; obtained from in vitro microsomal incubation in the current study.

; Metabolites of noribogaine, scaled maximum rate of metabolism $(\mu \mathrm{mol} / \mathrm{h})$

$\mathrm{Vmax} 2=\mathrm{Vmaxc} 2 / 1000 * 60 * \mathrm{MPL} * \mathrm{~L} * \mathrm{BW}$

; Metabolites of noribogaine, affinity constants ( $\mu \mathrm{mol} / \mathrm{L})$

$\mathrm{Km} 2=305$

; obtained from in vitro microsomal incubation in the current study.

; Run settings

; Molecular weight $(\mathrm{g} / \mathrm{mol})$

$\mathrm{MWibo}=310.4$

MWnor $=296.4$

; molecular weight of ibogaine

; molecular weight of noribogaine

; Given dose (mg/kg bw) and oral dose in $\mu \mathrm{mol} / \mathrm{kg}$ bw for ibogaine 
; Liver compartment

$;$ ALibo $=$ Amount ibogaine in liver tissue $(\mu \mathrm{mol})$

ALibo' $^{\prime}=\mathrm{QL} *($ CAibo - CVLibo $)+($ AGIibo * kaibo $)-$ AMLibo' -ABibo'

Init ALibo=0

$\mathrm{CLibo}=\mathrm{ALibo} / \mathrm{VL}$

CVLibo $=$ CLibo $/$ PLibo

;AMLibo=Amount ibogaine metabolized in liver to noribogaine

$$
\begin{aligned}
& \text { AMLibo' }=(\text { Vmax } 1 * \text { CVLibo }) /(\mathrm{Km} 1+\text { CVLibo }) \\
& \text { init AMLibo }=0
\end{aligned}
$$

; $\mathrm{ABibo}=$ amount of biliary excretion of ibogaine

ABibo' $=\mathrm{kbibo}^{*}$ ALibo

$$
\text { init } \mathrm{ABibo}=0
$$

; Kidney compartment

;AKibo $=$ Amount ibogaine in kidney tissue $(\mu \mathrm{mol})$

$$
\begin{gathered}
\text { AKibo' }^{\prime}=\mathrm{QK} *(\text { CAibo }- \text { CVKibo }) \\
\text { Init AKibo }=0 \\
\text { CKibo }=\text { AKibo } / \mathrm{VK} \\
\text { CVKibo }=\text { CKibo } / \text { PKibo }
\end{gathered}
$$

;Heart compartment

;AHibo $=$ Amount ibogaine in heart tissue $(\mu \mathrm{mol})$

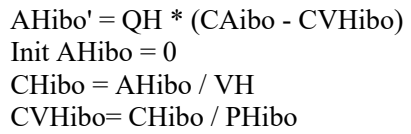

;Lung compartment

;ALuibo $=$ Amount ibogaine in lung tissue $(\mu \mathrm{mol})$

ALuibo' $=\mathrm{QC} *(\mathrm{CV}$ ibo - CALuibo $)$

Init ALuibo $=0$

CLuibo $=$ ALuibo $/ \mathrm{VLu}$

CALuibo $=$ CLuibo $/$ PLuibo

\section{; Arterial blood compartment}

;CAibo $=$ Concentration arterial blood ibogaine

$$
\begin{aligned}
& \text { AAibo' }^{\prime}=\mathrm{QC} *(\text { CALuibo- CAibo }) \\
& \text { Init AAibo }=0 \\
& \text { CAibo }=\text { AAibo } / \text { VA }
\end{aligned}
$$

; Venous blood compartment

;AVibo $=$ amount venous blood ibogaine $(\mu \mathrm{mol})$ 
AVibo' $=(\mathrm{QF} * \mathrm{CVFibo}+\mathrm{QR} * \mathrm{CVRibo}+\mathrm{QS} * \mathrm{CVSibo}+\mathrm{QL} * \mathrm{CVLibo}+\mathrm{QK} * \mathrm{CVKibo}+\mathrm{QH} * \mathrm{CVHibo}-$ QC * CVibo)

Init AVibo $=0$

$\mathrm{CVibo}=(\mathrm{AVibo} / \mathrm{VV})$

; Kinetics noribogaine sub-model

;Slowly perfused tissue compartment

; ASnor $=$ Amount noribogaine in slowly perfused tissue $(\mu \mathrm{mol})$

ASnor' $=$ QS * (CAnor- CVSnor)

Init ASnor $=0$

CSnor $=$ ASnor $/$ VS

CVSnor $=$ CSnor $/$ PSnor

; Rapidly perfused tissue compartment

; ARnor $=$ Amount noribogaine in rapidly perfused tissue $(\mu \mathrm{mol})$

$$
\begin{aligned}
\text { ARnor }^{\prime}=\mathrm{QR} * & \text { (CAnor }- \text { CVRnor }) \\
& \text { Init ARnor }=0 \\
& \text { CRnor }=\text { ARnor } / \text { VR } \\
& \text { CVRnor }=\text { CRnor } / \text { PRnor }
\end{aligned}
$$

; Fat compartment

; AFnor $=$ Amount noribogaine in fat tissue $(\mu \mathrm{mol})$

CFnor $=$ AFnor/ $\mathrm{VF}$

$$
\begin{aligned}
& \mathrm{AFnor}=\mathrm{QF} *(\mathrm{CAnor}-\mathrm{CVFnor}) \\
& \text { Init AFnor }=0
\end{aligned}
$$

CVFnor $=$ CFnor $/$ PFnor

; Uptake noribogaine from GI tract

;AGInor $=$ Amount noribogaine remaining in GI tract $(\mu \mathrm{mol})$

Init AGInor $=0$

AGInor' $=$ pulse $($ DOSEnor* Fanor, 0, dosenor_int $)+$-kanor * AGInor

; Liver compartment

; ALnor $=$ Amount noribogaine in liver tissue $(\mu \mathrm{mol})$

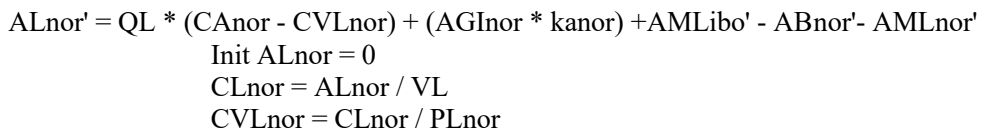

;AMLnor=Amount noribogaine metabolized in liver to noribogaine glucuronide

$$
\text { AMLnor' }=(\text { Vmax } 2 * \text { CVLnor }) /(\text { Km } 2+\text { CVLnor })
$$


init $\mathrm{AMLnor}=0$

; ABnor= amount of biliary excretion of noribogaine

ABnor'=kbnor*ALnor

init $\mathrm{ABnor}=0$

; Kidney compartment

; AKnor $=$ Amount noribogaine in kidney tissue $(\mu \mathrm{mol})$

AKnor' $=\mathrm{QK} *($ CAnor $-\mathrm{CVKnor})$

Init AKnor $=0$

$\mathrm{CKnor}=\mathrm{AKnor} / \mathrm{VK}$

CVKnor $=$ CKnor $/$ PKnor

; Heart compartment

; AHnor $=$ Amount noribogaine in heart tissue $(\mu \mathrm{mol})$

$$
\begin{aligned}
& \text { AHnor' }=\mathrm{QH} * \text { (CAnor- CVHnor }) \\
& \text { Init AHnor }=0 \\
& \text { CHnor }=\text { AHnor } / \text { VH } \\
& \text { CVHnor }=\text { CHnor } / \text { PHnor }
\end{aligned}
$$

;Lung compartment

; ALunor $=$ Amount noribogaine in lung tissue $(\mu \mathrm{mol})$

$$
\begin{aligned}
& \text { ALunor }=\mathrm{QC} *(\mathrm{CVnor}-\mathrm{CALunor}) \\
& \text { Init ALunor }=0 \\
& \text { CLunor }=\mathrm{ALunor} / \mathrm{VLu} \\
& \text { CALunor }=\text { CLunor } / \text { PLunor }
\end{aligned}
$$

; Arterial blood compartment

; CAnor $=$ Concentration arterial blood noribogaine $(\mu \mathrm{mol})$

AAnor' $=$ QC $*($ CALunor- CAnor $)$

Init AAnor $=0$

CAnor $=$ AAnor $/$ VA

; Venous blood compartment

; AVnor $=$ Amount venous blood noribogaine $(\mu \mathrm{mol})$

$\mathrm{AVnor}=(\mathrm{QF} * \mathrm{CVFnor}+\mathrm{QR} * \mathrm{CVRnor}+\mathrm{QS} * \mathrm{CVSnor}+\mathrm{QL} * \mathrm{CVLnor}+\mathrm{QK} * \mathrm{CVKnor}+\mathrm{QH} * \mathrm{CVHnor}-$ $\mathrm{QC} *$ CVnor)

Init $\mathrm{AVnor}=0$

CVnor= (AVnor/ VV)

; Mass balance calculations of ibogaine

Totalibo' $=$ pulse $($ DOSEibo *Faibo, 0 , doseibo_int $)$

init Totalibo $=1 \mathrm{E}-50$ 
Calculatedibo $=\mathrm{AFibo}+\mathrm{ASibo}+\mathrm{ARibo}+\mathrm{ALibo}+\mathrm{AVibo}+\mathrm{AAibo}+\mathrm{AGIibo}+\mathrm{AMLibo}+\mathrm{ALuibo}+\mathrm{AKibo}+$ AHibo+ABibo

ERRORibo $=(($ Totalibo - Calculatedibo $) /($ Totalibo $+1 \mathrm{E}-30)) * 100$

MASSBALibo $=$ Totalibo - Calculatedibo +1

; Mass balance calculations of noribogaine

Totalnor' $=$ AMLibo'+pulse (DOSEnor *Fanor, 0, dosenor_int)

init Totalnor $=1 \mathrm{E}-50+\mathrm{AMLibo}$

Calculatednor $=$ AFnor + ASnor + ARnor + ALnor + AVnor + AAnor + ALunor + AKnor + AHnor + ABnor +

AMLnor + AGInor

ERRORnor $=(($ Totalnor - Calculatednor $) /($ Totalnor $+1 \mathrm{E}-30)) * 100$

MASSBALnor $=$ Totalnor - Calculatednor +1

; Calculation with model

; Calculations to evaluate the model performance of ibogaine

CViboB $=$ CVibo $*$ MWibo

; Concentration of ibogaine in venous blood $(\mu \mathrm{g} / \mathrm{l})$

AUCibo' $=$ CViboB

; Calculate AUC for ibogaine

init $\mathrm{AUCibo}=0$

CVheartibo $=$ CVHibo $*$ MWibo

; Concentration of ibogaine in heart venous blood $(\mu \mathrm{g} / \mathrm{l})$

; Calculations to evaluate the model performance of noribogaine

CVnorB $=$ CVnor $*$ MWnor

; Concentration of noribogaine in venous blood $(\mu \mathrm{g} / \mathrm{l})$

AUCnor' $=$ CVnorB

; Calculate AUC for noribogaine

init $\mathrm{AUCnor}=0$

CVheartnor $=$ CVHnor*MWnor

; Concentration of noribogaine in heart venous blood (ug/l)

$\mathrm{BPribo}=2.5$

;blood to plasma ratio of ibogaine, assumed to be same as

noribogaine

BPrnor $=2.5$

fupibo $=0.04$

;blood to plasma ratio of noribogaine (Mash et al. 2016)

current study

fupnor $=0.26$

study

; fraction unbound in plasma of ibogaine obtained from the

;fraction unbound in plasma of ibogaine obtained from the current

; toxic equivalency factor based on in vitro cardiotoxic potency $\left(\mathrm{BMCL}_{10}\right.$ of ibogaine $=0.11 \mu \mathrm{M} \mathrm{BMCL}_{10}$ of noribogaine $=0.15 \mu \mathrm{M}$ ) obtained in the hiPSC-CM MEA assay in the current study.

TEFibo $=1$

TEFnor $=0.73$

; toxic equivalency concentration upon the oral exposure of ibogaine

fCVheartTEQ $=$ CVheartibo* (fupibo/BPribo) *TEFibo+CVheartnor* (fupnor/BPrnor) *TEFnor 


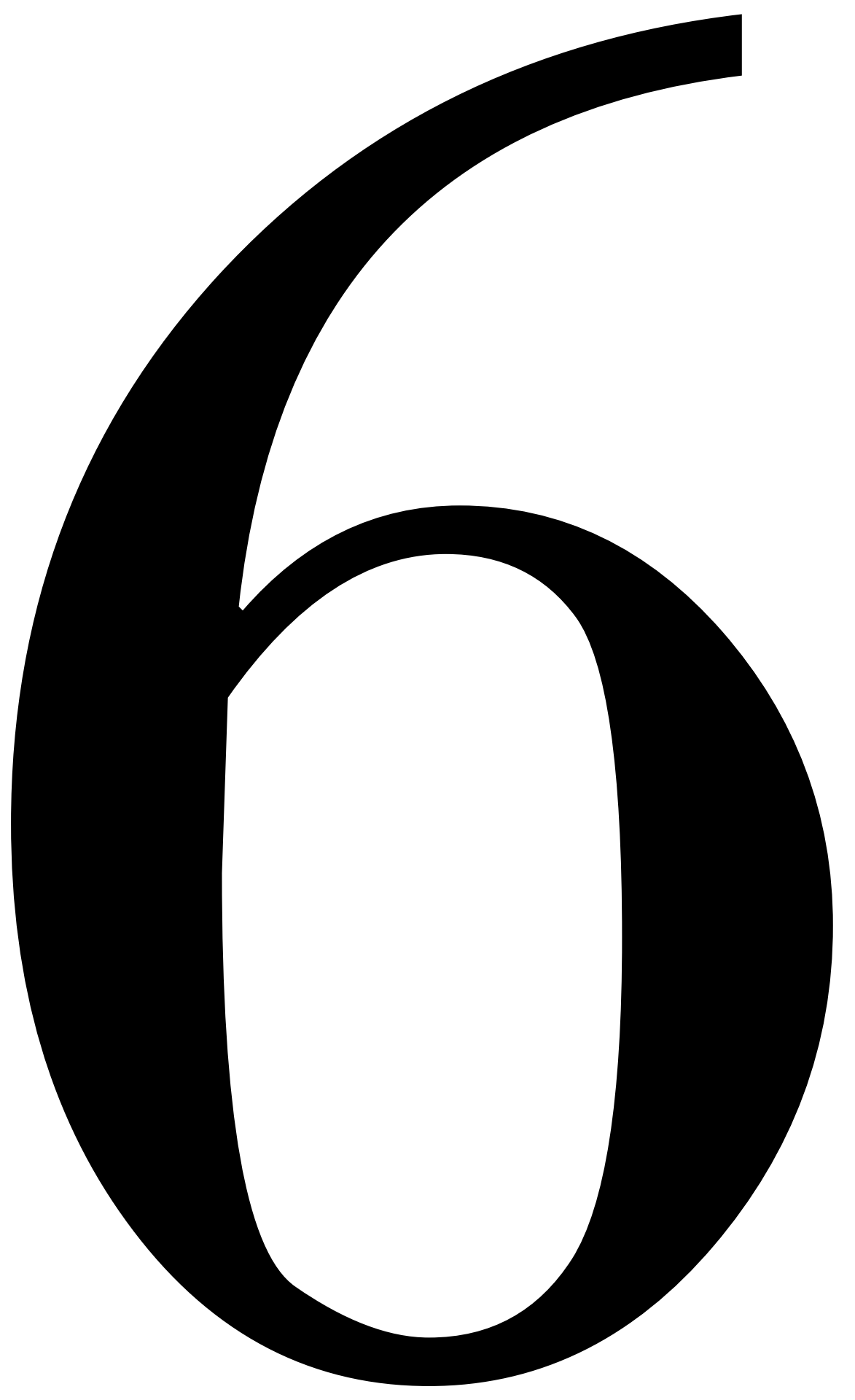


Chapter 6

General Discussion 


\section{Overview of the results and main findings}

In the last decades a paradigm shift can be observed in the toxicity testing used for chemical risk assessment and safety evaluation. The use of animal studies in toxicity testing is being debated. This is because of the inter-species differences between animals and human as well as the ethical and financial concerns related to animal studies. Therefore alternative testing strategies or new approach methodologies (NAMs) that integrate in vitro and in silico approaches are currently being developed.

As an important component of such NAMs, physiologically based kinetic (PBK) modeling can link the internal concentrations at the target organ with the external doses of the chemicals that humans are exposed to. The use of PBK modelling also enables extrapolation of in vitro data to the in vivo situation taking the toxicokinetics into account. A quantitative in vitro to in vivo extrapolation (QIVIVE) approach combining data from an in vitro toxicity assay and PBK modeling via so-called PBK modeling-based reverse dosimetry, has already adequately predicted the in vivo toxicity in experimental animals for various toxic endpoints in recent years including for example liver toxicity, nephrotoxicity, developmental toxicity and neurotoxicity (Abdullah et al. 2016; Algharably et al. 2021; Chen et al. 2019; Gilbert-Sandoval et al., 2020; Louisse et al. 2010 and 2017; Ning et al. 2019, Omwenga et al. 2021; Strikwold et al. 2013 and 2017; Zhao et al. 2019). To further facilitate the use of the PBK modeling-based reverse dosimetry approach for chemical risk assessment and safety evaluation it is of interest to extend its potential applicability to a broader range of toxicity endpoints and to the human situation. Considering that cardiotoxicity is one of the most important toxicity endpoints in safety testing of chemicals (non-pharmaceuticals and pharmaceuticals) and that lots of animals are needed for cardiac safety testing, animal free testing approaches are urgently needed for this endpoint. So far, proofs-of-principle for the PBK modeling-based reverse dosimetry approach mainly exist for the prediction of toxicity in experimental animals while ultimately predictions for human are needed. Therefore, the present thesis aims to provide proofs-ofprinciple for using PBK modeling-based reverse dosimetry of in vitro cardiotoxicity data for the quantitative prediction of cardiotoxicity in humans, thereby providing a new approach methodology in cardiac risk assessment and safety evaluations.

In Chapter 2, two stem cell-based in vitro models were evaluated for cardiotoxicity screening of chemicals. The first model, namely the mouse embryonic stem cell-derived cardiomyocyte (mESC-CM) model, used beating arrest as a toxicity readout and the second 
model, being the human induced pluripotent stem cell-derived cardiomyocyte (hiPSC-CM) multi-electrode array (MEA) assay, had multiple electrophysiological parameters as readout. To evaluate the two models, the cardiotoxicity data of eleven model compounds obtained in the two assays were compared. The results showed that the $\mathrm{mESC}-\mathrm{CM}$ beating arrest assay was not responsive to four hERG channel blockers and one $\mathrm{Na}^{+} / \mathrm{K}^{+}$ATPase inhibitor, being digoxin. Whereas the hiPSC-CM MEA assay was responsive to all hERG channel blockers and more sensitive to two sodium channel blockers and the $\mathrm{Na}^{+} / \mathrm{K}^{+}$ATPase inhibitor ouabain. The effective concentrations inducing $10 \%$ change in the readouts $\left(\mathrm{EC}_{10}\right)$ obtained in the hiPSCCM MEA assay were two to three orders of magnitude lower than those obtained in the mESCCM model. Additionally, the two models showed similar sensitivity to two calcium channel blockers and a $\beta$-adrenergic receptor agonist. The reason underlying the observed disparities could be partly attributed to 1) the inter-species differences in expression level, function and maturity of ion channels present in the mouse or human cardiomyocytes, 2) the differences in differentiation level of stem cells (i.e. combination of diverse cardiac cell types in the mouse cell model and a monolayer of ventricular cardiomyocytes in the human cell model) which could influence the diffusion of compounds to their targets in the cell models and 3) the temporal differences in endpoints used in two models (i.e. an early and late stage indicator of cardiotoxicity being electrophysiological alterations and beating arrest, respectively). Given that the hiPSC-CM MEA assay was more sensitive and had a broader compound coverage, a comparison was made between in vitro effective concentrations (i.e. $\mathrm{EC}_{10}$ ) obtained from the hiPSC-CM MEA assay and the reported serum concentrations associated with human (in vivo) observed responses observed in the electrocardiogram (ECG). This allowed the evaluation of the potential use of the human stem cell model for the prediction of human in vivo cardiotoxicity. Results revealed a good correlation between in vitro and in vivo data for most hERG channel blockers and sodium channel blockers with the differences being mostly smaller than 5-fold. Overall, our results showed that both models could be used to detect cardiotoxicity within the respective applicability domains. The mESC-CM beating arrest assay could be used as the first step in a tiered approach for cardiotoxicity screening to detect hazards related to cardiotoxicity via effects on for example sodium and calcium ion channels. The hiPSC-CM MEA assay was applicable to detect all evaluated compounds with different modes of action (MoAs) and thus could be used as a second tier to detect cardiotoxicity. Furthermore, given its adequate prediction of effective concentrations, and the use of human cells instead of cells of animal origin, it was selected as the model of choice as basis for QIVIVE predictions of human cardiotoxicity. 
Chapter 3 demonstrated that the combination of the hiPSC-CM MEA assay and PBK modeling based reverse dosimetry could adequately predict methadone-induced cardiotoxicity in humans. The in vitro cardiotoxicity of methadone and its metabolites EDDP and EMDP was quantified using the hiPSC-CM MEA assay. A human PBK model of methadone with a submodel of EDDP was developed using data obtained from in silico predictions, in vitro microsomal incubations and literature. The comparison between model predictions and the reported blood kinetic data showed a difference of less than 2-fold, indicating a good model performance and thus allowing the use of the developed PBK model for the reverse dosimetry. Both methadone and EDDP induced concentration-dependent prolongation of field potential duration corrected for beat rate (FPDc) in hiPSC-CMs. However, the unbound in vitro concentration of EDDP causing 20\% FPDc prolongation was respectively 41- and 12-fold higher than the unbound maximum blood concentration of EDDP reported in the subjects receiving an oral dose of methadone of $57.5 \mathrm{mg} /$ day and predicted using the developed submodel for EDDP at a high dose level of methadone of $200 \mathrm{mg} /$ day. These facts point at a limited contribution of cardiotoxicity of EDDP in the vivo situation. Thus, the cardiotoxicity of EDDP was not considered for the reverse dosimetry. Subsequently the in vitro concentration-response curve of methadone was converted to an in vivo dose-response curve for QTc prolongation in humans. Additionally, considering the variation in protein plasma binding of methadone reported in in vivo human studies, the PBK modeling based reverse dosimetry was performed using different unbound fraction $\left(f_{u b}\right)$ values of methadone. Results revealed that the prediction using high and low $f_{u b}$ values were well in line with data obtained from individual case studies and epidemiological population studies, respectively. The reason underlying this observation could be partly explained by the distinctive physiological and pathological conditions of subjects in the two types of studies, which could influence the plasma concentration of alphal-acid glycoprotein to which methadone is mainly bound in plasma thereby influencing the fraction of unbound methadone. Altogether, this chapter provides a proof-of-principle of using PBK modeling-based reverse dosimetry of in vitro cardiotoxicity data for the prediction of QTc prolongation in humans.

Results of the sensitivity analysis of the developed PBK model in Chapter 3 revealed that metabolism related parameters were highly influential on the methadone PBK model predictions and thus that the variation in metabolism would be a potential factor contributing to the interindividual human variation in the sensitivity towards the cardiotoxicity of methadone. This was further investigated in Chapter $\mathbf{4}$ where the developed PBK model of 
methadone was used as a basis to construct PBK models for R-and S-methadone for the Caucasian and Chinese population, and to study the inter-individual and inter-ethnic variability in the methadone enantiomer-induced cardiotoxicity. Instead of racemic methadone, the two enantiomers were used as model compounds because of their different potency in cardiotoxicity and stereoselective metabolism. To investigate the effect of inter-individual and inter-ethnic kinetic variations on the cardiotoxicity of the two methadone enantiomers in the Caucasian and the Chinese population, two sources of metabolic variation data were incorporated in the PBK models. In the first approach the metabolic variation was characterized using kinetic constants obtained from in vitro incubations with 25 Caucasian and 25 Chinese individual human liver microsomes (HLMs) to define 50 individual PBK models by which blood kinetics of the two methadone enantiomers in the two populations were predicted. In the second approach reported kinetic constants for the conversion of the two enantiomers by recombinant cytochrome P450 isoforms (rCYPs) and variations in CYP abundances were incorporated in the PBK models and combined with Monte Carlo simulations to predict the probability distribution of blood kinetics of the two methadone enantiomers in the two populations. The results showed that both approaches similarly predicted the inter-individual and inter-ethnic variations in the kinetics of the two enantiomers. A higher catalytic efficiency for the metabolism of both enantiomers and thus lower predicted blood concentrations of the enantiomers at similar dose levels with a higher coefficient of variation were observed in the Caucasian population compared to the Chinese population. This may partly be due to the reported inter-ethnic differences in functional alleles and the abundance of CYPs involved in the metabolism as well as in the content of cytochrome b5 that provides electrons for the CYP mediated conversions. Subsequently, the predicted blood kinetics obtained using rCYPs combined with Monte Carlo simulation were used to derive chemical specific adjustment factors (CSAFs), which were further applied to define dose-response curves obtained by reverse dosimetry for sensitive individuals within the populations. BMD analysis and the Margin of Safety (MOS) approach were subsequently used to evaluate the inter-ethnic difference in sensitivity towards R- and S-methadone. The results revealed that Chinese may be at relatively higher risk towards the cardiotoxicity of methadone with MOS values at similar dose levels being 2-fold lower than those for Caucasians for both methadone enantiomers. In conclusion, this chapter illustrated that integrating in vitro cardiotoxicity and metabolic data, PBK modelling and Monte Carlo simulation can be a powerful approach to predict the role of kinetics in inter-ethnic and inter-individual variation in cardiotoxicity, which can be used to refine the cardiac risk assessment and safety evaluation. 
Given that cardiotoxicity is an important endpoint not only in drug development with respect to pharmaceuticals but also for public health with respect to food-related and environmental chemicals, it is of great value to explore the potential applicability of the developed NAM to predict human cardiotoxicity for a broader range of compounds. This is needed to prove that the adequate prediction of in vivo methadone-induced cardiotoxicity is not a unique case. Therefore, Chapter 5 investigated the possibility of the PBK modeling-based reverse dosimetry approach to predict the in vivo cardiotoxicity of the herbal alkaloid ibogaine and its metabolite noribogaine. Following a similar procedure as the one presented in Chapter 3, the cardiotoxicity of ibogaine and noribogaine was quantified in vitro using the hiPSC-CM MEA assay. PBK models to predict the toxicokinetics of ibogaine and noribogaine in human were developed using parameters obtained from in silico methods and the literature, and biokinetic data were obtained from a Caco-2 transport study and in vitro liver microsomal incubations. Using the developed PBK model, in vitro concentration-response curves (from the hiPSC-CM MEA assay) were translated to in vivo dose-response data for QTc prolongation using PBK modeling-based reverse dosimetry. Results of the hiPSC-CM MEA assay showed that both ibogaine and noribogaine prolonged FPDc in a concentration-dependent manner with ibogaine being 1.4-fold more potent than noribogaine. Unlike what was observed for the methadone metabolite EDDP in Chapter 3, the unbound in vitro effective concentration of noribogaine inducing $10 \%$ prolongation of FPDc was within the range of the unbound maximum blood concentration of noribogaine after ingestion of clinically relevant doses of ibogaine (typically $500-1000 \mathrm{mg}$ ). Thus, the cardiotoxicity of noribogaine was taken into account in the reverse dosimetry for the prediction of ibogaine-induced cardiotoxicity by using the toxic equivalency (TEQ) approach using toxic equivalency factors (TEFs). Given that the oral administration of noribogaine is reported to be associated with QTc prolongation in humans, reverse dosimetry of in vitro cardiotoxicity of noribogaine was performed to predict the in vivo noribogaineinduced QTc prolongation. Comparison of both model predictions to reported in vivo data showed that the developed approach adequately predicted the cardiotoxicity of both ibogaine and noribogaine in human. Additionally, the relative contribution of ibogaine and noribogaine in ibogaine-induced cardiotoxicity was investigated by integrating the TEQ approach in the PBK model. It was shown that noribogaine plays a substantial role in the in vivo cardiotoxicity upon oral administration of ibogaine. A relatively smaller contribution of ibogaine itself to the in vivo cardiotoxicity could be ascribed to its low unbound fraction in plasma and its extensive metabolism. In conclusion, this chapter provided an additional proof-of-principle for using 
PBK modeling-based reverse dosimetry of hiPSC-CMs MEA assay data for the prediction of human cardiotoxicity, which can be used for risk assessment and safety evaluation.

\section{General discussion and future perspectives}

The present thesis demonstrated the potential of PBK modeling-based reverse dosimetry of in vitro data to predict human cardiotoxicity reflected by effects on QTc prolongation, providing a NAM for the cardiac risk assessment and safety evaluation of chemicals. The obtained results will be further discussed to elucidate considerations on study limitations, applications and future improvements, including the following topics:

- Applicability domains of in vitro cardiotoxicity models

- Considerations for the PBK model

- Use of PBK modeling-based reverse dosimetry

- Implications for risk assessment

- Future perspectives

\subsection{Applicability domains of in vitro cardiotoxicity models}

The present thesis first focused on two stem cell-based in vitro models and provided a comprehensive evaluation of their capacities to detect chemical-induced cardiotoxicity via different mechanisms by using eleven model compounds, and thereby elucidated the potential for use of the two models for cardiotoxicity screening and QIVIVE. The following section presents additional considerations related to the proposed applicability domains of the two in vitro cardiotoxicity models.

\subsubsection{Applicability domain of the mESC-CM beating arrest assay}

Considering the fundamental differences of the readouts of the two cell models (i.e. beating arrest vs. electrophysiological parameters) and the species differences for the cell species used (i.e. mouse vs. human), it is of importance to point out that the aim of Chapter 2 was not to quantitatively compare the two models, but to evaluate their applicability domains and explore their potential as a model for cardiotoxicity screening and/or for QIVIVE. Given the increasing demand for evaluation of chemicals, including pharmaceuticals, industrial chemicals, natural alkaloids, environmental pollutants and other potentially cardiotoxic chemicals (Burnett et al. 2021; Kratz et al. 2017; Krishna et al. 2020), it is essential to consider an in vitro model that is suitable for screening of a large number of chemicals. For such aim it can be advantageous that compounds have a similar MoA in human and mice, but at the same time it is not a prerequisite. In this case, apart from biological considerations, costs and practical aspects should also be 
taken into account for the evaluation, and with respect to these topics the mouse model may have advantages. The $\mathrm{mESC}-\mathrm{CMs}$ are easy to obtain, and the $\mathrm{mESC}-\mathrm{CM}$ beating arrest assay is cost-friendly and relatively easy to implement, which may result in the mESC-CM beating arrest assay to be a first-choice candidate for cardiotoxicity screening, as long as it is kept in mind that it may not detect for example hERG channel blockers.

The results of chapter 2 demonstrated that the mESC-CM beating arrest assay detected the cardiotoxicity of sodium and calcium channel blockers and could be used as a first step in a tiered approach as a first screen for detection of hazards related to cardiotoxicity via effects on these ion channels. The mESC-CM beating arrest assay was not responsive to the hERG channel blockers tested, while hERG channel blockers are considered problematic compounds, since blockage of hERG channels is a frequently encountered off-target activity during drug development, associated with prolonged QTc interval and thus potentially causing lifethreatening ventricular arrythmia (Martin et al. 2004; Sanguinetti et al. 1995; Thomas et al. 2006). The observed low sensitivity of the mESC-CM towards hERG channel blockers could be attributed to several reasons. It has been reported that $h E R G$ channel-mediated rapid delayed rectifier potassium currents $\left(\mathrm{I}_{\mathrm{kr}}\right)$ represent one of the major currents involved in the regulation of repolarization in human ventricular cardiomyocytes, while it is not a prominent current in mouse cardiomyocytes (Nerbonne 2004; Xu et al. 1999). The repolarization of mouse cardiomyocytes is mainly regulated by three other types of delayed rectifier potassium currents,

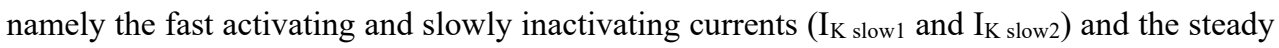
state current $\left(\mathrm{I}_{\mathrm{ss}}\right)$, that are not expressed in human ventricular cardiomyocytes (Huang 2017; $\mathrm{Xu}$ et al. 1999). Thus, the observed low sensitivity of the mouse model towards hERG channel blockers could be the result of a relatively small contribution of hERG channels to the regulation of repolarization in mouse ventricular cardiomyocytes.

Furthermore, the use of beating arrest as the readout may be another reason for the low sensitivity of the mouse model. Beating arrest is considered as a late marker of cardiotoxicity that follows some electrophysiological alterations (e.g. changes in beat rate and time duration of repolarization) occurring at an early stage. Additionally, beating arrest may not be an indicative endpoint when chemical-induced electrophysiological alterations would not result in the cessation of beating of the cells. Many studies explored the potential of using mESCCMs combined with electrophysiological approaches for cardiotoxicity detection. Dofetilide, a typical hERG channel blocker used in the present thesis, was reported to change contraction amplitude and beating rate in a concentration-dependent manner when analyzing impedance 
signals from mESC-CMs (Himmel 2013). Ikeuchi et al. (2015) differentiated mESC to the beating cells following the same approach as used in the present thesis. The effects of chemical exposure on beating cells were recorded and the obtained videos were converted to data on inter-beat interval and beat rate using an optical microscopy imaging system (Ikeuchi et al. 2015). Indeed, using electrophysiological parameters as the readout appears to improve the applicability of mESC-CMs. However, measurement and analysis of those parameters require specialized expertise and equipment, thereby losing some of the major advantages of the assay as a simple and cheap assay.

Besides the considerations for the mouse model on hERG channel blockers and endpoints, mESC-CMs also show dissimilarities in certain morphology features of the action potential compared to human cardiomyocytes (Danik et al. 2002; Huang 2017; Kaese and Verheule 2012), indicating that special caution and carefully interpretation are needed when extrapolating mouse data to the human situation, and that the predictive or translational value may be considered limited. Therefore, the use of the mESC-CMs beating arrest assay may not be adequate for QIVIVE to predict human cardiotoxicity.

\subsubsection{Applicability domain of hiPSC-CM MEA assay}

In Chapter 2 of the present thesis it was concluded that the hiPSC-CM MEA assay provides a suitable in vitro model for QIVIVE for predicting human cardiotoxicity because of the high sensitivity and good in vitro-in vivo concordance in unbound effective concentrations and affected endpoints. Chapter 2 showed that the hiPSC-CM MEA assay responded to model compounds with diverse MoAs and the obtained effective concentrations were highly concordant with in vivo effective concentrations especially for hERG channel and sodium channel blockers. Furthermore, the electrophysiological parameters used in hiPSC-CM MEA assay were considered as suitable readouts to reflect human in vivo clinical endpoints. Namely, extracellular field potentials of hiPSC-CMs as measured in the MEA correlated well to action potentials of hiPSC-CMs measured by the patch clamp technique and are considered to some extent correlated with human ECG parameters (Sala et al. 2017; Tertoolen et al. 2018). Especially, in the clinic, exposure to hERG channel blockers caused a prolongation of the QT interval which reflected the ventricular action potential duration (APD) (Hondeghem and De Clerck 2012). Considering a good linear relationship observed between APD and the field potential duration (FPD) with an $\mathrm{R}^{2}$ of 0.999 (Tertoolen et al. 2018), hERG channel blockerinduced prolongation of FPD in the hiPSC-CM MEA assay can be seen as the surrogate for the QT interval prolongation in the ECG (Zwartsen et al. 2019). Many studies also demonstrated 
that the prolongation of FPD corrected for beat rate (FPDc) and the occurrence of in vitro arrhythmia-like waveforms in the MEA assay have high translational value for clinical cardiotoxicity (Blinova et al. 2018; Millard et al. 2018). This was corroborated by the results obtained in Chapter 3 and 5 where with the concentration-dependent effects of methadone and (nor)ibogaine on FPDc prolongation their dose-dependent effects on QTc prolongation in human in vivo were adequately predicted.

As mentioned above the hiPSC-CM MEA assay showed great promise for cardiotoxicity screening and QIVIVE. To further refine the application of the model and interpretation of results, future efforts could focus on improving the maturity of hiPSC-CMs and optimizing experimental settings. These suggestions are indicated by the following considerations. hiPSCCMs are generally considered as a powerful predictive tool for drug proarrhythmic risk testing and disease modelling (Crumb Jr et al. 2016; Pourrier and Fedida 2020). It is well documented that the electrophysiological properties of most key ion channels in hiPSC-CMs such as $\mathrm{I}_{\mathrm{Ca}, \mathrm{L}}$, $\mathrm{I}_{\mathrm{Kr}}$ and $\mathrm{I}_{\mathrm{Ks}}$. remarkably resemble those of human cardiomyocytes (Barbuti et al. 2016; Karakikes et al. 2015). On the other hand, it is reported that certain characteristics of hiPSCCMs are different from the ones of adult cardiomyocytes, while being more similar to fetal cardiomyocytes with e.g. higher density of pacemaker current $\left(\mathrm{I}_{\mathrm{f}}\right)$ (Guo et al. 2011; Hoekstra et al. 2012; Pourrier and Fedida 2020). Unlike the hiPSC-CMs, human adult ventricular cardiomyocytes do not exhibit spontaneous beating and their contractions are triggered by impulses transmitted via a cardiac conduction system where If plays an essential role in initiating the spontaneous beating. The current density of $\mathrm{I}_{\mathrm{f}}$ in hiPSC-CMs was reported to be higher compared to the $\mathrm{I}_{\mathrm{f}}$ density in human adult ventricular cardiomyocytes at a similar membrane potential (Baruscotti et al. 2010; Ma et al. 2011). The robust $\mathrm{I}_{\mathrm{f}}$ in hiPSC-CMs prevented the complete repolarization to the resting potential and thus was considered to contribute to the spontaneous beating of hiPSC-CM (Hoekstra et al. 2012; Ma et al. 2011). Such a dissimilarity may lead to a complicated in vitro-in vivo comparison as the $\mathrm{I}_{\mathrm{f}}$ modulation would be a confounding factor for the in vitro-in vivo correlation of chemical-induced chronotropic effects observed in hiPSC-CM (Pang et al. 2019). In the present thesis the potential influence of the immature phenotype on the characteristics of hiPSC-CMs was to some extent overcome by culturing the hiPSC-CMs in medium that was specifically designed for improving maturity of hiPSC-CMs as demonstrated by similar electrophysiological properties and gene expression pattern to adult cardiomyocytes (Mulder et al. 2018). Additionally, many other differentiation methods have been developed to improve the maturity 
of hiPSC-CMs through biophysical stimulations (Nunes et al. 2013), optimized growth substrates (Patel et al. 2015), medium additives (Yang et al. 2014), extended culture time (Rajamohan et al. 2013) or three-dimension culture (Sirenko et al. 2017).

Furthermore, given that MEA chips with a six-well format (i.e. allowing only six independent treatments at the same time) were used in the present thesis, like in most hiPSCCM MEA studies, exposure to the compounds was performed in a cumulative dosing manner. This enables testing of a wider chemical concentration range and the direct comparison of treatments to the same baseline. On the other hand, this may also bring some limitations. For example, the cumulative dosing may have influence on the observed effects given that certain compounds can accumulate in the cell membrane and/or cytosol and may result in the (de)sensitization of ion channels and/or receptors, such as in the case of catecholaminesinduced $\beta$-adrenergic receptor desensitization (Lohse et al. 1996; Uzun et al. 2016). To further elucidate the potential difference between a cumulative and single dosing pattern, the effective concentrations $\left(\mathrm{EC}_{10}\right)$ of compounds obtained in the present thesis were compared to the available data (for dofetilide, mexiletine, nifedipine and isoproterenol) obtained from hiPSCCM MEA studies which employed a single dosing strategy (Zwartsen et al. 2019). Comparisons revealed that the two dosing patterns resulted comparable type of effects of the compounds (i.e. increase or decrease) with a less than 2 -fold difference between the $\mathrm{EC}_{10}$ values obtained in the present thesis using cumulative dosing and reported concentrations inducing around $10 \%$ change on MEA readouts upon single dosing. This difference was even smaller than the observed inter-laboratory variations when using the same dosing approach. Likewise, our data on noribogaine obtained via single or cumulative dosing showed that the results were comparable, demonstrating a comparable potency in prolongation of FPDc (Figure 1). It appears that the influence of cumulative dosing was relatively insignificant for the compounds discussed above, however, it should be noted that the accumulation of compounds is dependent on their physicochemical properties and the potential effects on ion channel/ receptor (de)sensitization may differ from case to case. Given that comprehensive information on mechanisms and effects is not available for most compounds tested in the hiPSC-CM MEA assay, single dosing experiments could be considered to avoid possible interference, particularly when the use of a higher-throughput platform of MEA (e.g. 96-well plate) is possible, which could ensure an efficient detection for a larger number of samples thereby facilitating testing a wide concentration range by single dosing. 


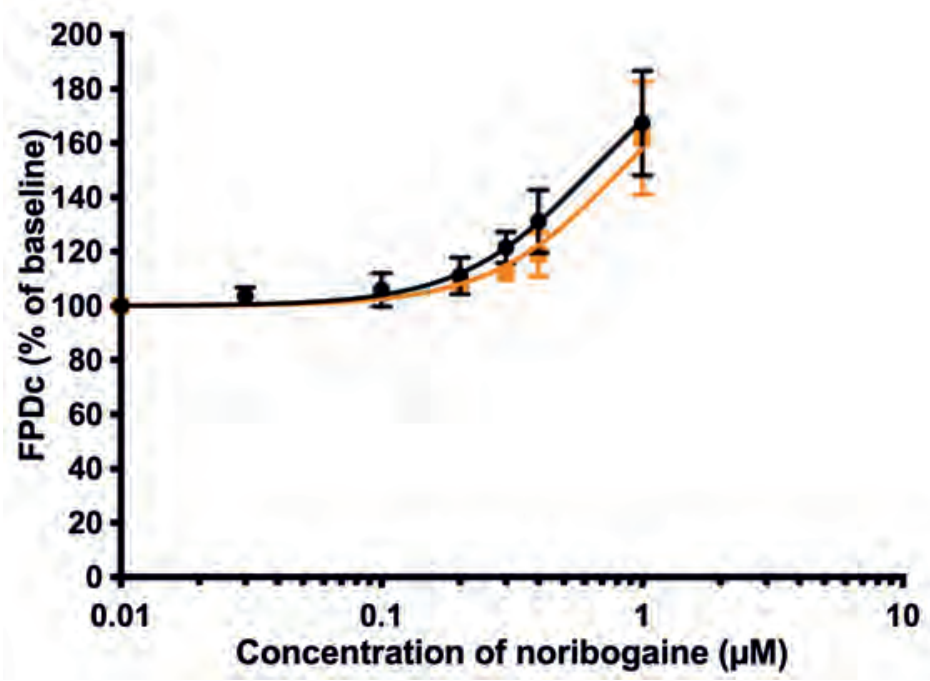

Figure 1 Concentration-response curves for the effect of noribogaine on FPDc in hiPSC-CM detected by the MEA using cumulative dosing (black circles and line) or single dosing (orange squares and line). The response of the baseline condition $(0.1 \%(\mathrm{v} / \mathrm{v}) \mathrm{DMSO})$ was set at $100 \%$. Data represent the mean of results obtained from three independent experiments each containing six well replicates for the cumulative dosing and from two independent experiments each containing one or two well replicates for the single dosing.

\subsection{Considerations for the PBK model}

Chapter 3, 4 and 5 demonstrated how in vitro cardiotoxicity data obtained from the hiPSC-CM MEA assay can be translated to in vivo cardiotoxicity dose-response curves by using PBK modeling-based reverse dosimetry. Being the key step of the approach, PBK modeling fills the gap on kinetics between in vitro data and the in vivo situation. The accuracy of the PBK model influences the applicability of the model for its intended purpose, which in this case is reverse dosimetry to predict in vivo cardiotoxicity. In this section topics related to model building and evaluation and refinements for model predictions are discussed.

\subsubsection{Scaling of in vitro metabolism parameters for PBK models}

For chemicals that are extensively metabolized, an accurate measurement and description of their metabolism is essential for developing PBK models because of the large impact of metabolism on the toxicokinetics and bioactivation/detoxification of the chemicals (Fisher et al. 2020). In the present thesis two frequently used in vitro systems including liver microsomes and recombinant cytochrome P450 enzymes (rCYPs) were employed to determine the metabolic constants (the Michaelis-Menten constant $\mathrm{K}_{\mathrm{m}}$ and the maximum rate of the reaction 
$\left.\mathrm{V}_{\max }\right)$. Subsequently, the obtained in vitro metabolic constants were translated to the in vivo situation to allow their use in the PBK models developed in Chapter 3, 4 and 5. Such scaling was achieved by using different scaling factors in both models. For liver microsomes a simple scaling factor can be applied to scale the $\mathrm{V}_{\max }$ by correcting for differences in the amount of microsomal protein in the HLM and the intact liver. For supersomes expressing single rCYP, the extrapolation of a rCYP dependent rate constant to the in vivo situation is more complicated since an additional step is needed to first scale the rCYP system to liver microsomes, so that by using the scaling factor for liver microsomes, the rate constant can be scaled to the in vivo situation (Brandon et al. 2003; Ooka et al. 2020; Wetmore et al. 2014). The scaling from the rCYP system to liver microsomes can be done by using a so-called inter-system extrapolation factor (ISEF). The ISEF for a specific CYP can be calculated by dividing the $\mathrm{V}_{\max }$ of a CYP probe substrate measured in HLM ( $\mathrm{pmol} / \mathrm{min} / \mathrm{mg}$ microsomal protein) by the $\mathrm{V}_{\max }$ of that CYP probe substrate measured in the $\mathrm{rCYP}$ system ( $\mathrm{pmol} / \mathrm{min} / \mathrm{pmol} \mathrm{CYP}$ ) that is divided by the abundance of that CYP in the HLM samples (pmol CYP/ mg microsome protein) (Proctor et al. 2004). Within the ISEF, differences in CYP abundances between both systems are cancelled out by a correction for the CYP abundance in the HLM used. This makes ISEF values dimensionless and allow the consideration of differences in the intrinsic activity per amount of the CYP of interest between the two systems (Proctor et al. 2004; Crewe et al. 2011), reflecting the differences in lipid environment, nonspecific binding, and concentration of accessory proteins (Crewe et al. 2011; Lipscomb and Poet 2008). By using the variation in CYP abundances in a population, the ISEF allows the scaling of the $\mathrm{V}_{\max }$ of the compound of interest measured in rCYP system to study the population variability in metabolism as demonstrated in Chapter 4.

Generally, the ISEF value for a specific CYP is derived based on in vitro metabolic data of a suitable specific probe substrate. The obtained ISEF value is often considered as a "standard" value and used to predict total metabolic clearance of a compound metabolized by this CYP. However, the obtained prediction is not always comparable to the in vivo data or to the ones predicted using liver microsome data, and the discrepancy could be partly attributed to the over/underestimation of the ISEF values (Badhan et al. 2019; T'jollyn et al. 2015; Youdim et al. 2008). Several studies have demonstrated that the estimation of ISEFs could be influenced by many factors including the selection of the probe substrate, the recombinant systems used and the concentration of accessory proteins (e.g. cytochrome b5). Umehara et al. (2017) found that the ISEFs for CYP3A4 differed from 0.06 to 0.35 among seven probe substrates and a large variation in ISEFs for CYP2C9 derived using different probe substrates was also reported 
(Chen et al. 2011; Crewe et al., 2011). The observed substrate-dependent effects of ISEF estimations may be partly explained by heterogeneities of probe substrate binding towards the active site of the relevant CYP (Kumar et al. 2006; Stresser et al. 2000; Williams et al. 2003; Umehara et al. 2017). Additionally, the ISEFs for the same probe substrate could be different when different recombinant enzyme systems are used. For example, the ISEF for bufuralol (CYP2D6 probe substrate) obtained using baculovirus transformed insect cells was 67-fold lower compared to the ISEF obtained using B-lymphoblastoid cells (Proctor et al. 2004). It is also reported that the level of accessory proteins can have an important role in the ISEFs since ISEFs for CYP2C9 differed up to 10-fold between rCYP systems with and without cytochrome b5 (Crewe et al. 2011), the latter being involved in providing the electron for the CYP catalysis.

Chapter 4 of the present thesis demonstrated a compound-specific approach to derive ISEFs for the two methadone enantiomers for which metabolic data were obtained from incubations with human liver microsomes and a rCYPs system (Baculovirus-insect cells) and the fraction metabolized by each CYP. The use of compound specific ISEFs enables avoiding the substratedependent effect on the ISEF calculations mentioned above and subsequently on model predictions. The prerequisite for defining these compound-specific ISEFs is the availability of information on the fraction metabolized by each CYP in HLM of the compound of interest, which information may not be available for newly synthesized/discovered compounds and additional experiments would be needed. To further mitigate the potential influence of variations in ISEF on the extrapolation, probe substrate derived ISEFs could be corrected to ISEFs specific for the compound of interest by fitting the prediction with experimental human data (Badhan et al. 2019). However, such a correction would not be the first option since it can be only applied when there are in vivo data available. Other methods, such as using the average of ISEFs for different probe substrates (Chen et al., 2011) also contribute to more accurate ISEFs. Moreover, deriving rCYP system specific ISEFs (with probe substrates) can be recommended. This can be done by using the same rCYP system (same batch is preferred) that is used to quantify the CYP specific metabolism of the compound of interest and using HLMs that adequately resemble the individual or population of interest, i.e. with respect to relevant cytochrome b5 levels.

\subsubsection{The role of protein binding in hepatic metabolism}

In addition to an adequate extrapolation of in vitro metabolism parameters, a suitable recognition of the influence of protein binding in hepatic metabolism is also important for developing an accurate PBK model. Generally, there are three types of protein binding that 
may be considered in the prediction of hepatic metabolism, namely plasma protein binding, tissue protein binding in the intracellular space, and non-specific binding of chemicals to components of the in vitro incubation systems used (e.g. liver microsomes and hepatocytes) (Benet and Zia-Amirhosseini 1995; Deb et al. 2018; Heuberger et al. 2013; Kalvass et al. 2001; OCED 2021; Kotsiou and Tesseromatis 2011; Sweeney and Gearhart 2020). It is of importance to consider to what extent the protein binding could influence the prediction of hepatic metabolism and how it depends on the properties of the chemical of interest, and also that the correction for one or more types of protein binding does not always contribute to improving the in vivo predictions of hepatic metabolism (Heuberger et al. 2013; Fagerholm 2007; Nichols et al. 2018; Obach et al. 1999). This was corroborated by the results in the current thesis where the metabolism algorithm in the PBK models of methadone and ibogaine did not correct for protein binding (so called unrestricted clearance, assuming that the chemical bioavailability to metabolizing enzymes in the in vitro and in vivo situation is effectively the same), but still properly predicted in vivo metabolism, as reflected by the accurate PBK model prediction of blood kinetics. This was also observed in the study of Obach et al. (1999) where the in vivo clearance values were predicted without adding a correction for binding in plasma and to liver microsomes. These predictions were reported to be more accurate or comparable to the in vivo data than the ones predicted by including all binding factors for lipophilic basic and neutral compounds (Obach et al. 1999). The reason underlying such observations remains unclear but it was assumed to be the result of similar unbound fractions in the in vivo and the in vitro system for the metabolism of these chemicals (Poulin et al. 2002). Additionally, plasma protein binding was considered to highly influence the hepatic clearance of so-called restrictive clearance chemicals, for which clearance is restricted by strong binding to plasma proteins hampering binding to the respective enzymes. On the other hand, clearance of non-restrictive clearance chemicals is unlikely to be affected by plasma protein binding (Ye et al. 2016).

It should also be noted that this does not mean that protein binding is not important and can be ignored when considering hepatic metabolism, particularly in the case where the unbound fraction of chemicals may be very different between the extracellular and intracellular situation. For example, it has been suggested that for some ionizable compounds, ionic interactions between the extracellular protein-chemical complex and the hepatocyte surface and the differences in $\mathrm{pH}$ between plasma and intracellular water may result in a different unbound chemical concentration in liver compared to plasma. Thus using the unbound fraction in liver $\left(f_{u}\right.$, liver $)$ would be expected to better predict in vivo hepatic clearance (Berezhkovskiy 2011; 
Burczynski et al. 2001; Poulin et al. 2012). This was supported by Poulin et al. (2012) who demonstrated that a more accurate prediction of in vivo hepatic clearance for some chemicals highly bound to plasma protein can be obtained using in vitro metabolism data that take into account the ionization of chemicals and $\mathrm{f}_{\mathrm{u}}$, liver, compared to the prediction only corrected for plasma protein binding and/or non-specific binding in incubation systems. Furthermore, Obach et al. (1999) also demonstrated that the inclusion of binding to plasma protein and microsomes to some extent improved the prediction of in vivo hepatic clearance for acidic compounds. Overall, it would be prudent to always carefully consider if it is essential to account for different binding conditions and types of protein binding when considering the use of in vitro metabolic data for in vivo metabolism predictions. It is also important to note that the correction for protein binding should also be considered when performing the reverse dosimetry. The need for a correction for differences in protein binding in the in vitro bioassay and the in vivo situation when performing reverse dosimetry will be discussed in section 2.3.1.

When protein binding is considered necessary to be integrated in the prediction of hepatic metabolism (and in the reverse dosimetry), the unbound fraction values can be determined by various in vitro and/or in silico approaches. Many approaches have been developed to determine the plasma protein binding including in vitro models such as equilibrium dialysis (Ye et al. 2017; van Liempd et al. 2011) and ultrafiltration (Howard et al., 2010; Wang and Williams 2013), as well as many in silico structure-based prediction models (Moroy et al. 2012; Vallianatou et al. 2013; Wageningen Food Safety Research (WFSR) 2020). Furthermore, the non-specific binding of chemicals to human liver microsomes, hepatocytes and/or proteins in the incubation medium can be predicted using in silico models which require information on microsome concentration and on the physicochemical properties of the compound of interest (Gao et al. 2008; Hallifax and Houston 2012; Kilford et al. 2008; Poulin and Haddad 2011) while it can also be experimentally determined using equilibrium dialysis and ultrafiltration (Barr et al. 2019; Gao et al. 2008). Compared to plasma protein binding, information on tissue (liver) binding is more challenging to obtain in vitro, given the difficulty to access tissues and the fact that in vitro measurements would require intact organs, tissue homogenates, or incubated tissue slices (Brunner and Langer 2006; Riccardi et al. 2018). In silico tools using mathematical algorithms could also indirectly estimate the unbound concentration of chemicals in the liver from $\mathrm{f}_{\mathrm{u}, \mathrm{p}}$ (Poulin and Theil 2002). 


\subsubsection{Refinement of the description of excretion in the PBK model}

Besides the hepatic metabolism, the present thesis also considered the importance of excretion and included urinary and/or biliary excretion in the PBK models as routes for the elimination and clearance of model compounds and their metabolites when relevant. The renal and biliary excretion would be expected to influence the blood kinetics of EDDP given that a large percentage of EDDP originating from a given dose of methadone was reported to clear via urine and feces (Foster 2001; Kharasch et al. 2004; Kharasch et al. 2009). Similarly, biliary excretion showed a high influence on the predicted blood kinetics of noribogaine with a sensitivity coefficient of the biliary excretion rate constant being 0.25 . In the light of these facts, the inclusion of excretion for these compounds would be necessary for an accurate prediction on their blood kinetics, especially for noribogaine for which the biliary excretion is the predominant elimination route. In the present thesis parameters for renal and biliary excretion of methadone, EDDP and noribogaine were either taken from reported in vivo data or derived by fitting the predicted blood kinetics to respective reported human data. However, these approaches are restricted to compounds with available in vivo data. Therefore, to better comply with the $3 \mathrm{R}$ principles and enable the prediction of excretion for chemicals lacking in vivo data, it would be valuable to integrate in vitro-derived biliary or urinary excretion parameters in the PBK model. The description of excretion can be further refined by using algorithms with different complexity, allowing the consideration for multiple mechanisms involved in the excretion of chemicals of interest.

The simplest model for renal elimination is to assume that chemicals can be cleared directly from the blood or kidney compartments into the urine and that all processes involved in the renal excretion can be represented by an overall rate of renal excretion (Quindroit et al. 2019; Mukherjee et al. 2014; Zhang et al. 2007). The rate of renal excretion can be described as a first-order process using the intrinsic clearance $(1 / h)$ or rate constant $(/ h)$ for renal clearance, or as a saturable process expressed in Michaelis-Menten kinetics (Haddad and Nong 2020). More complex models have been developed to describe the multiple processes involved in the renal excretion, allowing a detailed look on glomerular filtration, active transport in proximal tubular cells and passive diffusion (Worley and Fisher 2015; Dubbelboer et al. 2017). More recently, Huang and Isoherranen (2018) demonstrated that a generic dynamic physiologically-based mechanistic kidney model developed based on human physiology can adequately predict the renal clearance for 40 compounds with active renal transport using the plasma unbound fraction, 
in vitro transporter uptake clearance and in vitro permeability data obtained from the MadinDarby canine kidney (MDCK) or Caco-2 cell line.

Comparable to the equations used to describe urinary excretion, biliary excretion can be described in an empirical model using the rate of biliary excretion from the liver following either first-order or Michaelis-Menten kinetics (Nong et al. 2009; Noorlander et al. 2021a). Physiologically based mechanistic models are available for the prediction of fecal elimination and enterohepatic circulation (Clewell et al. 2008). Noribogaine was suspected to undergo enterohepatic circulation since fluctuations in the noribogaine concentration-time curves during the distribution phase were observed in certain individuals upon oral administration of noribogaine (Glue et al. 2015). However, given that such fluctuations were not observed in the mean noribogaine concentration-time profiles in human clinical studies (Glue et al. 2015 and 2016) and the fact that the developed PBK model of noribogaine including the biliary excretion without enterohepatic circulation correctly predicted the blood kinetics required for reverse dosimetry (see results in Chapter 5), the enterohepatic circulation of noribogaine might not substantially influence the first phase of the blood concentration-time profile in humans from which the $\mathrm{C}_{\max }$ for the reverse dosimetry is derived. The inclusion of enterohepatic circulation in the noribogaine PBK model may still be valuable to explain the slow in vivo elimination observed at later time points and provide further insight into the ADME of noribogaine.

Many in vitro cell-based models have been developed to determine kinetic parameters for renal and biliary excretion, being the input for the models mentioned above. Transfected cells with specific transporter(s), such as Chinese hamster ovary (CHO) cells, MDCK cells, human embryonic kidney 293 cells (HEK293) and pig kidney epithelial cells (LLC-PK1), have been widely used to investigate the active excretion of chemicals via kidneys or liver in vitro (Hirano et al. 2005; Matsushima et al. 2005; Zhang et al. 2012). By using transfected MDCK and HEK cells, Campbell et al. (2015) found that EDDP but not methadone was the substrate for several key uptake transporters including organic-anion transporting polypeptides (OATP1A2 and OATP1B1) and organic cation transporters (OCT1 and OCT3) and for efflux transporters including P-glycoprotein and breast cancer resistant protein (BCRP). Besides, bi-directional transport assays employing immortalized liver (e.g. HepaRG), kidney (e.g. Caki) and intestine (e.g. Caco-2) cell lines with the expression of functional transporters, as well as hepatocytebased models are also well developed methods to study the excretion of chemicals (Brantegem et al. 2019; Giacomini et al. 2010; Soldatow et al. 2013; Van Zhang et al. 2012). No matter which in vitro assay is performed to derive kinetic parameters of excretion, it is of importance 
to consider the differences in expression level and activity of transporters between in vitro models and in vivo organs and thus adequate scaling factors are needed to translate the in vitro determined values to in vivo relevant input parameters for PBK models (Choi et al. 2019; Haddad and Nong 2020; Noorlander et al. 2021 a and b).

\subsection{Use of PBK modeling-based reverse dosimetry}

An adequate prediction of in vivo cardiotoxicity requires careful consideration for each key step involved in the entire PBK modeling-based reverse dosimetry approach. The importance of in vitro model selection, in vivo resemblance of the in vitro endpoint and the PBK model establishment were discussed in the previous sections. This section focuses on the relevant choices with respect to the extrapolations from in vitro toxicity data to in vivo toxicity values, including the selection of dose metric together with a correction for differences in protein binding and inclusion of metabolites in the reverse dosimetry.

\subsubsection{Selection of dose metric for the in vitro in vivo extrapolation}

The selection of an appropriate dose metric is an important step to quantitatively predict in vivo effects from in vitro toxicity data. The maximum concentration $\left(\mathrm{C}_{\max }\right)$ and the area under the blood or plasma concentration-time curve (AUC) are two often used dose metrics. For selecting the appropriate dose metric, the time-depend nature of the toxic effect and MoA of the chemical should be taking into account. It has been proposed that the AUC could be used for chemicals causing irreversible cumulative toxic effects, such as genotoxicity or carcinogenicity. Alternatively, $\mathrm{C}_{\max }$ would be a suitable dose metric for the chemicals with reversible mechanisms (Groothuis et al. 2015). In Chapter 3 and 5 of the thesis, the recovery of cardiotoxicity (i.e. relief of prolonged FPDc) was observed for the model compounds studied (methadone, EDDP, ibogaine and noribogaine) when exposure medium was replaced by fresh medium, indicating a reversible reaction to be involved in the blockage of ion channels by these model compounds, and thus $\mathrm{C}_{\max }$ was considered a more appropriate dose metric used for the QIVIVE.

In the present thesis, in vitro concentration-response curves were translated to in vivo doseresponse curves by assuming unbound in vitro concentrations to be equal to unbound concentrations in the heart venous blood. The assumptions on in vitro and in vivo dose metrics involved in this translation were as follows. Based on a well-accepted notion that only the unbound fraction of a chemical can distribute to the site of action and cause toxicity (Deb et al. 2018; Howard et al. 2010), a correction for differences in protein binding in the in vitro model 
and the in vivo situation should be considered in the reverse dosimetry. The most relevant dose metric for the in vitro toxicity assay to be used as input for the reverse dosimetry would be the unbound intracellular concentration at the site of action or the membrane concentration (Groothuis et al. 2015; Fisher et al. 2019). Many studies have emphasized the importance of measurement or prediction of intracellular concentrations for QIVIVE (Armitage et al. 2014; Hamon et al. 2015), which is also evident from a study by Poulin et al. (2012) already mentioned in section 2.2.2. These authors demonstrated that the use of unbound intracellular liver concentrations can improve the accuracy of predicted in vivo hepatic clearance especially for chemicals highly bound to plasma protein. However, determination or prediction of intracellular concentrations is challenging and often practically not feasible (Albrecht et al. 2019). For example, dilution, homogenization and incubation involved in preparing tissue samples for experiments on tissue protein binding like equilibrium analysis may disrupt the intracellular components (e.g. acidic organelles) that contribute to the in vivo distribution of chemicals (Clausen and Bickel 1993; Kotsiou and Tesseromatis 2011). This may result in an underprediction of in vivo distribution of basic lipophilic chemicals including methadone to liver and kidney when using the in vitro binding values obtained from liver and kidney homogenates (Clausen and Bickel 1993; Kotsiou and Tesseromatis 2011). Intracellular concentrations used in in vitro assays can also be estimated by some mathematic-based models, which however require several prerequisites for the prediction, such as the fact that they are only applicable for neutral and unionized compounds (Armitage et al. 2014; Comenges et al. 2017; Worth et al. 2017) or a steady-state assumption of multiple dynamic processes (Armitage et al. 2014; Fischer et al. 2017). On the other hand, the selection for an internal concentration should also consider whether the increased accuracy of the prediction is worth the investment of performing additional experiments (Groothuis et al. 2015). Given that adequate QIVIVE can be obtained by using unbound extracellular concentrations in the in vitro cardiotoxicity assay medium, as shown in Chapter 3 and 5, the use of unbound extracellular concentrations for the QIVIVE can be a first-choice dose metric. When the use of unbound extracellular concentrations does not work or when intracellular exposure is known to be significantly different from extracellular exposure in certain cases such as protein (albumin)-facilitated uptake of chemicals in hepatocytes (Bowman and Benet 2018; Burczynski et al. 2001), an attempt can be made to use (unbound) intracellular concentrations as the dose metric for QIVIVE, despite the fact that measurement of these intracellular concentrations is a challenge. 
It is common practice in QIVIVE to link the in vivo plasma concentration to in vitro concentrations (Wetmore et al. 2015). Plasma dosimetry, the unbound concentration in heart venous blood, was used as the in vivo dose metric for QIVIVE in the present thesis. This was based on an assumption that the unbound concentration in heart venous blood reflects the concentrations at the site of action, which is supported by the study of Mikkelsen et al. (2018). By using the postmortem concentrations of methadone in biophysically based mathematical models of human cardiac electrophysiology, the authors demonstrated that methadone induced QTc prolongation was best predicted based on unbound plasma concentrations whereas the use of both unbound and total heart tissue concentrations resulted in an overpredicted effect on the QTc (Mikkelsen et al. 2018).

\subsubsection{Role of metabolites in reverse dosimetry}

The in vivo toxicity of a chemical may be caused by the parent chemical itself and/or its metabolite(s). When there is no in vivo toxicity information available for metabolites, the determination of their in vitro toxicity is of importance to identify their role in the chemicalinduced toxicity as well as to ensure an adequate prediction of the in vivo toxicity of the parent chemical. This was illustrated by the results in Chapter 3 and 5 of the present thesis where two cardiotoxic metabolites EDDP and noribogaine, being the primary metabolites of respectively methadone and ibogaine, were shown to play different roles in their parent compound-induced in vivo cardiotoxicity. The findings obtained in theses chapters also provided insight into the factors that influence the relevance of cardiotoxicity of metabolites in the reverse dosimetry for the prediction of parent chemical-induced cardiotoxicity, such as the relative potency and internal concentration of the metabolite relative to those of the parent compound. Additionally, the adequate prediction of ibogaine-induced cardiotoxicity in Chapter 5 demonstrated that the in vitro-PBK modeling-based approach integrated with the TEQ approach provided a strategy to predict in vivo cardiotoxicity of a chemical for which also the metabolites are active.

In Chapter 3 and 5, the cardiotoxicity of EDDP and noribogaine were detected in the hiPSCCM MEA assay. Results showed that both EDDP and noribogaine induced concentrationdependent prolongation of FPDc with a 4- and 1.3-fold lower potency than methadone and ibogaine, respectively. However, only the cardiotoxicity of noribogaine was taken into account in the reverse dosimetry of ibogaine while the cardiotoxicity of EDDP did not need to be included for the reverse dosimetry of methadone. As aforementioned, this was based on the fact that the unbound in vitro effective concentrations of EDDP were substantially higher than its internal unbound concentrations upon the oral administration of methadone while the 
unbound in vitro effective concentrations of noribogaine were within the range of its unbound internal blood concentrations after clinically relevant dosing of ibogaine. This indicates that in addition to positive in vitro cardiotoxic effects, the relevance of in vitro cardiotoxicity to the in vivo situation needs to be considered to justify the inclusion (or not) of cardiotoxicity of metabolites in the reverse dosimetry for the prediction of parent chemical-induced cardiotoxicity. When no in vivo data for the metabolite are available to compare with its unbound in vitro effective concentrations, a PBK submodel for the metabolite can be developed to provide such data for comparison.

When the cardiotoxicity of metabolites is considered relevant, the combined effective concentration of the parent chemical and its active metabolite(s) can be described as the equivalent concentration expressed either in parent chemical or metabolite equivalents. The unbound concentration in parent compound or metabolite equivalents subsequently can be used as the dose metric in the reverse dosimetry for the prediction of parent (or metabolite) chemicalinduced cardiotoxicity using the in vitro data for the parent compound or the metabolite, the latter depending on the choice for expressing the equivalent concentrations. Such a TEQ approach can be achieved by using the toxic equivalency factor (TEF) or relative potency factor (RPF). These two types of relative potency factors can be considered similar given that both TEF and RPF values can be used in the TEQ approach to assess the combined effects of chemical mixtures taking the potency of each mixture component into account (Bil et al. 2021; Bosgra et al. 2009; EFSA 2013; EFSA 2019; Safe 1998; van Ede et al. 2016; WHO 2016;). The RPF values can be used for mixture components with the same toxic effects which may result from different MoAs given that information on the MoA is often lacking, whereas the establishment of TEF values requires more information on the MoA and could be considered as a specific type of RPF with the prerequisite of mixture components sharing the same MoA (Bil et al. 2021; EFSA 2013; EFSA 2019; U.S. Environmental Protection Agency (EPA) 2000). In the present thesis these two terms were not specifically distinguished and the TEF was used as the general term. Besides the prerequisite on 1) similar MoAs and/or toxicological effects, the use of the TEF based approach also requires that 2) mixture components only differ in potency (i.e. show similar shape of the individual concentration-response curves on a log-scale) and 3 ) their toxicity are concentration (dose) additive (i.e. no synergism or antagonism) (Bil et al. 2021; Bosgra et al. 2009; EFSA 2019; Safe 1998). To what extent ibogaine and noribogaine fulfill these assumptions is discussed below. 
The fulfillment of the first requirement is supported by the fact that both ibogaine and noribogaine can prolong QTc in human (Glue et al. 2016; Hoelen et al. 2009) and FPDc in hiPSC-CMs as observed in the present thesis, which may be ascribed to their blockage of hERG channels observed in in vitro studies employing the patch clamp technique (Alper et al. 2016; Koenig et al. 2014; Rubi et al. 2017). The fulfillment of the second requirement follows from the statistical comparison of the hillslope values of the concentration-response curves of ibogaine and noribogaine (Figure 4 in Chapter 5), which showed that the hillslope values of ibogaine and noribogaine were comparable (with a $\mathrm{p}$ value of 0.07 ), indicating that the two concentration-response curves were parallel. Given that limited data are available for the cardiotoxicity of ibogaine and noribogaine especially on their combination effects, an assumption was made that cardiotoxic effects of ibogaine and noribogaine were additive to enable the application of the TEF based approach in the present thesis. This assumption to some extent could be considered as adequate since the predicted cardiotoxicity of ibogaine using the TEF based approach appeared to match well with the reported in vivo cardiotoxicity of ibogaine (Figure 8 in Chapter 5). One may argue that possible synergism or antagonism could exist between the two compounds. However, since the unbound internal blood concentration of ibogaine was predicted to be approximately 100-fold lower than that of noribogaine upon a clinically relevant dose of ibogaine (calculated based on the data shown in Chapter 5), the presence of ibogaine would not be expect to influence the cardiotoxic potency of noribogaine in the in vivo situation. Whether this also holds for the effects of noribogaine on ibogaine toxicity remains to be established. Nevertheless, it would be of interest to experimentally verify whether the cardiotoxicity of the two compounds is additive. To this end, the combined effect of ibogaine and noribogaine can be assessed by detecting the FPDc prolongation induced by an equipotent mixture of the two compounds. The obtained concentration-response curve of the mixture expressed in ibogaine equivalents needs then to be comparable to the one of ibogaine alone to support additive cardiotoxicity.

\subsection{Implications for risk assessment}

In traditional risk assessment involving in vivo animal studies, points of departure (PoDs) derived from animal data to define safe exposure levels of chemicals for humans are generally divided by a default uncertainty factor of 100 that comprises the default factors of 10 for interspecies and 10 for intraspecies (inter-individual) differences (IPCS 2005). However, the use of default uncertainty factors may be over- or under-protective resulting in over- or underestimation of the risk of chemical use, and either unnecessary restrictions for chemicals 
or insufficient protection for the sensitive subpopulation (Kasteel and Westerink 2021; Zeise et al. 2013). Thus, to refine the risk assessment of chemicals, these default uncertain factors could be replaced by chemical-specific adjustment factors (CSAFs) defined based on chemical specific data (IPCS 2005). By integrating the variation of input data (both kinetic and dynamic), the developed PBK modeling-based reverse dosimetry approach offers the potential to characterize the interspecies and inter-individual variation and refine some of the default uncertainty factors used in the risk assessment. Chapter 4 shows how PBK modeling-based reverse dosimetry can be combined with Monte Carlo simulation to quantify the interindividual kinetic variation underlaying possible variation in cardiotoxicity of the two methadone enantiomers, and to define CSAFs for interindividual and inter-ethnic kinetic differences for the Caucasian, Chinese and combined populations. Results obtained showed that the default CSAF of 3.16 for interindividual kinetic differences appeared to adequately cover the inter-individual differences in toxicokinetics for $\mathrm{R}$ - and S-methadone-induced cardiotoxicity in the Caucasian and Chinese population. When considering the combined population, the default CSAF was just sufficient for R-methadone while for S-methadone the CSAF for kinetic differences amounted to 4.5 and 4.7 for protection of the $95^{\text {th }}$ and $99^{\text {th }}$ percentile of the population, respectively.

It is of importance to note that the CSAF for kinetic differences derived in Chapter 4 was based only on the metabolic variations for both methadone enantiomers given that large interindividual variations in methadone pharmacokinetics have been reported to be the result of variability in methadone metabolism (Eap et al. 2002). In the present approach the CSAF for kinetic differences did not yet take into account variability in other factors that influence the kinetics of the methadone enantiomers, such as body weight and oral fraction absorbed, which were parameters of major influence illustrated in the sensitivity analysis in Chapter 3 and 4. Variation in the fraction unbound in plasma also influences the kinetics of methadone as shown in Chapter 3. To evaluate this further for this discussion chapter the PBK modeling combined with Monte Carlo simulation was used to predict the kinetic variations based on the variations in metabolism, body weight, oral fraction absorbed and fraction unbound in plasma of R- and S-methadone. Monte Carlo simulation was performed in the same way as described in Chapter 4. The coefficients of variation for these parameters were assumed to be 0.3 representing a moderate level of variation (Covington et al. 2007). Table 1 shows the results thus obtained and reveals that CSAF values for kinetic variations increased when considering the variation in body weight, fraction absorbed and fraction unbound in plasma. Similar to the CSAF derived 
based on metabolic variations (values in brackets in Table 1), the default CSAF of 3.16 would still be adequate to cover inter-individual differences in kinetics for the Caucasian and the Chinese population except for protection of the $99^{\text {th }}$ percentile of the Caucasian population in the case of exposure to S-methadone for which a CSAF of 3.3 for inter-individual kinetic differences would be required. For the combined population the default CSAF ranged from 4.5 to 8.3 for protection of the $95^{\text {th }}$ and $99^{\text {th }}$ percentile of the population, respectively. This implies that kinetic differences of methadone enantiomers among human individuals, especially for Smethadone, are higher than the default value of 3.16 for inter-individual kinetic differences. For protecting the $99^{\text {th }}$ percentile of the human population for the exposure to S-methadone, the CSAF of 8.3 defined for inter-individual kinetic differences (Table 1), together with the default uncertainty factor of 3.16 for inter-individual dynamic differences, would result in a CSAF for inter-individual differences of 26.2, which is 2.6-fold higher than the default uncertainty factor of 10 for human inter-individual differences consisting of default uncertainty factors of 3.16 for human variability in both toxicokinetics and toxicodynamics (IPCS, 2005). Thus, an increase of the uncertainty factor would be needed to sufficiently protect the human population from S-methadone induced cardiotoxicity.

Table 1 CSAFs of R- and S-methadone for the Caucasian population, the Chinese population and the two populations combined in each scenario of Monte Carlo simulation taken into account variation in metabolism, bodyweight and oral fraction absorbed.

\begin{tabular}{|c|c|c|c|c|c|c|}
\hline & \multicolumn{3}{|c|}{ CSAFs at $95^{\text {th }}$ percentile } & \multicolumn{3}{|c|}{ CSAFs at $99^{\text {th }}$ percentile } \\
\hline & $\begin{array}{c}\text { Caucasian } \\
\text { population } \\
\text { a }\end{array}$ & $\begin{array}{c}\text { Chinese } \\
\text { population }^{\mathrm{a}}\end{array}$ & $\begin{array}{c}\text { Two } \\
\text { populations } \\
\text { combined }^{\mathrm{b}}\end{array}$ & $\begin{array}{l}\text { Caucasian } \\
\text { population }{ }^{\mathrm{a}}\end{array}$ & $\begin{array}{c}\text { Chinese } \\
\text { population }^{\mathrm{a}}\end{array}$ & $\begin{array}{c}\text { Two } \\
\text { populations } \\
\text { combined }^{\mathrm{b}}\end{array}$ \\
\hline $\begin{array}{c}\text { R- } \\
\text { methadone }\end{array}$ & $2.4(1.7)$ & $1.9(1.2)$ & $4.5(3.0)$ & $3.1(2.0)$ & $2.5(1.3)$ & $5.8(3.2)$ \\
\hline $\begin{array}{c}\text { S- } \\
\text { methadone }\end{array}$ & $2.5(1.9)$ & $2.1(1.3)$ & $6.7(4.5)$ & $3.3(2.3)$ & $2.6(1.4)$ & $8.3(4.7)$ \\
\hline
\end{tabular}

Values in brackets were obtained in Chapter 4 where the CSAF values were derived based on the metabolic variations. ${ }^{\text {a }}$ obtained by dividing the $95^{\text {th }}$ or $99^{\text {th }}$ percentile of the $C_{\max }$ in heart venous blood by the GM of the $C_{\max }$ in heart venous blood in each population. ${ }^{\mathrm{b}}$ obtained by dividing the $95^{\text {th }}$ or $99^{\text {th }}$ percentile of the $\mathrm{C}_{\max }$ in heart venous blood in the Chinese population as the most sensitive population by the GM of the $\mathrm{C}_{\max }$ in heart venous blood in the Caucasian population.

In addition to toxicokinetic variation, the variation in toxicodynamics can also play an important role in the inter-individual variation (Grimm et al. 2018; Zeise et al., 2013). To improve the developed QIVIVE approach for the prediction of inter-individual variation, it 
would be valuable to also consider the variations in toxicodynamics. In the context of electrophysiological cardiotoxicity, toxicodynamic variations could refer to the variation in baseline characteristics and responses to the chemical exposure, which may result from genetic variability (Britton et al. 2017; Grimm et al. 2018). HiPSC-CMs derived from healthy individuals with diverse genetic backgrounds have been demonstrated as a promising model to investigate the inter-individual variability in chemical-induced cardiophysiologic effects (Burnett et al. 2019; Grimm et al., 2018). By using such a population-based hiPSC-CM model with Bayesian modeling, Blanchette et al. (2020) estimated the uncertainty factor of 136 drugs and environmental chemicals for variations in different dynamic endpoints such as QT prolongation, positive (negative) chronotropy and cytotoxicity. The results demonstrated that 31 compounds showed QT prolongation effects and the inter-individual variability observed using the population-based hiPSC-CM model with Bayesian modeling was for all compounds higher than the default value of 3.16. This indicates that the further refinement of the uncertainty factors for toxicodynamic variations might be needed for the chemicals that can induce QT prolongation, such as hERG channel blockers. Given that the CSAFs for toxicokinetic differences of the methadone enantiomers were higher than the default uncertainty factor for toxicokinetic differences and that the CSAF for toxicodynamic differences of methadone, being a hERG channel blocker, might also be higher that the default uncertainty factor, it can be concluded that for methadone a CSAF for overall inter-individual variation higher than 10 would be needed.

Additionally, it should be noted that the PoDs derived in the present thesis using an in vitroPBK modeling-based approach are based on a human in vitro model for cardiotoxicity and a human PBK model. This implies that the use of such PoDs in risk assessment would not require the uncertainty factor for inter-species differences. Instead, an extra uncertainty factor could be considered because use of an in vitro-PBK modeling-based approach to define safe exposure level of chemicals for humans brings inherent uncertainties related to for example the in vitro toxicity assay used, the PBK model and its parameters and in the choice of the dose metric for QIVIVE.

\subsection{Future perspectives}

The present thesis provides proofs-of-principle for using PBK modeling-based reverse dosimetry of in vitro cardiotoxicity for the prediction of electrophysiological cardiotoxicity in humans. On the basis of findings in the present thesis, the developed in vitro-PBK modelingbased approach could be further extended to a broader range of toxicological endpoints and 
chemicals, which ultimately will contribute to the use of non-animal based NAMs for the human risk assessment and safety evaluation of chemicals. This can be achieved by optimizing and specializing the in vitro and/or in silico parts involved in the developed approach in two ways. One may consider a switch of the QIVIVE approach either to generic approaches that suit the need for rapid interpretation of data generated from high-throughput in vitro assays for large numbers of chemicals, or to more sophisticated approaches that integrate in vitro assays and PBK models adapted for diverse purposes. From such sophisticated approaches comprehensive compound specific information can be obtained and are appropriate for more accurate risk assessment. The combination of in vitro-PBK modeling with Monte Carlo simulation to investigate inter-individual variations and to define CSAFs as present in Chapter 4 is an example for the latter application.

For the first application (i.e. being generic approaches), by using a higher-throughput platform of the MEA (e.g. 96-well plate) with automated analysis programs (Kraushaar and Guenther 2019), the hiPSC-CM MEA assay could gain the potential to rapidly screen and identify the cardiotoxicity for large numbers of chemicals. To extrapolate such high amount of in vitro effective data to the in vivo scenarios for risk prioritization or assessment, efficient PBK modelling approaches are necessary (Yoon 2020). In this context, the development of a generic modeling framework would accelerate the efficiency of PBK modeling, which could be achieved by a streamlined model parameterization (Daga et al. 2018; Yoon 2020). Some efforts in developing generic PBK modeling based reverse dosimetry with simplified parameterization have been reported. The U.S. EPA initiated a strategy where high-throughput screening data of ToxCast and Tox 21 chemicals can be extrapolated by using generic PBK models which only included a few kinetic processes with limited experimental parameters such as unbound fraction in plasma, hepatic metabolic clearance, and intestinal permeability (Sipes et al. 2017; Wetmore et al. 2012 and 2015). The development of high-throughput and automated biokinetic assays to derive these parameters would further contribute to the efficiency of model parameterization. Furthermore, a free available web-based tool to build generic PBK models for rats and human has been developed and can be used to predict the internal concentrations upon chemical exposure by using limited input information including physicochemical parameters (i.e. $\log \mathrm{P}, \mathrm{pKa}$ and molecular weight), intestinal uptake and in vitro hepatic clearance (Punt et al. 2021). Combined with in vitro ToxCast bioactivity data (e.g. cytotoxicity, inhibition of human thyroid peroxidase activity and estrogenicity), the developed generic PBK model tool has been demonstrated to predict the oral equivalent doses for four 
food additives which were subsequently compared with their human exposure data for the risk assessment (Punt et al. 2021). Additionally, Zhang et al. (2018 and 2020) demonstrated that a generic PBK model combined with in vitro estrogenicity data can predict the in vivo uterotrophic response (i.e. increase of uterus weight) for seven compounds in rats. In their studies the model parameters related to the absorption (i.e. in vitro and in vivo permeability coefficient) and distribution (i.e. tissue/blood partition coefficients) were determined by in silico quantitative structure activity relationship (QSAR) (Hou et al. 2004; Sun et al. 2002) and quantitative property relationship (QPPR) approaches (DeJongh et al. 1997), respectively. In vitro incubations using liver S9 with all co-factors involved in phase I and phase II metabolism was performed to determine the overall hepatic clearance. Admittedly, a simplified model parameterization may more benefit from in silico approaches than in vitro experiments, in particular for the metabolic parameters given that the derivation of metabolic parameters in vitro was considered to be low throughput compared to the pace of data generation from in vitro toxicity assays (Yoon 2020). In summary, to enable the rapid interpretation of in vitro toxicity data, future efforts could be focused on developing more generic PBK models.

Furthermore, the application of the in vitro-in silico approach can be expanded to a more sophisticated compound-specific risk assessment, which enables the establishment of accurate PoDs, supporting the higher tier risk-based decision making of chemicals (Andersen et al. 2019; Yoon 2020). This can be done, for example, by including additional relevant toxicity endpoints in the QIVIVE approach. As aforementioned, cardiotoxicants may affect both cardiac electrical and contraction function via different mechanisms (Ovics et al. 2020). When combined with MEA or other techniques, hiPSC-CMs can be used to detect not only electrophysiological but also contractile (i.e. beat rate, contractile force), and even structural (i.e. subcellular structure morphology, cytotoxicity, mitochondrial dysfunction) effects of chemicals (Burnett et al. 2021; Chaudhari et al. 2016; Lee et al. 2021), which offers the opportunity to predict dose-response behavior for other relevant in vivo cardiotoxicity endpoints using PBK modeling-based reverse dosimetry. Recently, Li et al. (2021) demonstrated that the combination of PBK modeling and in vitro cardiotoxicity assays that detected doxorubicin-induced mitochondrial toxicity, cardiac arrhythmicity and cytotoxicity provided reasonable predictions of the PoDs of in vivo doxorubicin-induced cardiotoxicity. This work shows the promise of developing a QIVIVE approach to predict multiple in vivo endpoints. PBK modeling-based reverse dosimetry of in vitro data obtained from a multiparametric in vitro cardiotoxicity assay would provide a more comprehensive risk assessment of cardiotoxicity for a chemical of interest, which enables 
determination of the sensitive target/key cardiotoxic effect based on PoDs predicted for different endpoints.

Another example of combination of the in vitro-in silico approach with specific type of vitro cardiotoxicity data for a more sophisticated application could be the risk assessment of chemicals for specific/sensitive populations. The availability of hiPSC-CM derived from individuals with a specific genetic and demographic background (e.g. ethnic, life stage, gender) or from patients with cardiac diseases such as dilated cardiomyopathy (Panopoulos et al. 2017; Sun et al. 2012) and long QT syndromes (Egashira et al. 2012; Itzhaki et al. 2011; Shinozawa et al. 2017) could provide representative toxicodynamic data for sensitive subgroups within the population. The PBK models developed in the present thesis provide an adequate starting point for such studies. Moreover, PBK models can consider specific physiological conditions such as age-dependent enzyme activities (Mallick et al. 2020; Yang et al. 2006; Yang et al. 2019), pregnancy (Ke et al. 2014) and autoinduction of enzyme activity upon repeated exposure (Badhan et al. 2019).

\section{Conclusion}

The present thesis demonstrated that integration of in vitro cardiotoxicity data, in vitro kinetic data and PBK modeling adequately predicted human in vivo dose-dependent cardiotoxicity of two selected anti-addiction drugs. Furthermore, the present thesis demonstrated the use of PBK models to predict the inter-ethnic and inter-individual variations taking methadone enantiomers as examples, which showed the possibility to refine the uncertainty factors for inter-individual differences in toxicokinetics used in risk assessment. The obtained results subsequently can be used to refine the risk assessment for chemical-induced cardiotoxicity. Additionally, the QIVIVE approach was shown to adequately predict in vivo cardiotoxicity for a chemical with a bioactive metabolite, that should be taken into account based on its relative potency and in vivo concentration compared to the parent compound. Altogether, the present thesis provides proofs-of-principle for using PBK modeling-based reverse dosimetry of in vitro data for the prediction of cardiotoxicity in humans, further validating the potential applicability of this in vitro-in silico QIVIVE approach for a broader range of toxicity endpoints and for the human situation, ultimately contributing to non-animal based NAMs for risk assessment and safety evaluation of chemicals. 


\section{References}

Abdullah R, Alhusainy W, Woutersen J, et al. (2016) Predicting points of departure for risk assessment based on in vitro cytotoxicity data and physiologically based kinetic (PBK) modeling: the case of kidney toxicity induced by aristolochic acid I. Food and Chemical Toxicology 92:104-116

Albrecht W, Kappenberg F, Brecklinghaus T, et al. (2019) Prediction of human drug-induced liver injury (DILI) in relation to oral doses and blood concentrations. Archives of toxicology 93(6):1609-1637

Algharably EAe-H, Di Consiglio E, Testai E, et al. (2021) Prediction of the dose range for adverse neurological effects of amiodarone in patients from an in vitro toxicity test by in vitro-in vivo extrapolation. Archives of Toxicology:1-10

Alper K, Bai R, Liu N, et al. (2016) hERG blockade by iboga alkaloids. Cardiovascular toxicology 16(1):14-22

Andersen ME, McMullen PD, Phillips MB, et al. (2019) Developing context appropriate toxicity testing approaches using new alternative methods (NAMs). ALTEX-Alternatives to animal experimentation 36(4):523-534

Armitage JM, Wania F, Arnot JA (2014) Application of mass balance models and the chemical activity concept to facilitate the use of in vitro toxicity data for risk assessment. Environmental science \& technology 48(16):9770-9779

Badhan RK, Gittins R, Al Zabit D (2019) The optimization of methadone dosing whilst treating with rifampicin: A pharmacokinetic modeling study. Drug and alcohol dependence 200:168-180

Barbuti A, Benzoni P, Campostrini G, Dell'Era P (2016) Human derived cardiomyocytes: a decade of knowledge after the discovery of induced pluripotent stem cells. Developmental Dynamics 245(12):1145-1158

Barr JT, Lade JM, Tran TB, Dahal UP (2019) Fraction unbound for liver microsome and hepatocyte incubations for all major species can be approximated using a single-species surrogate. Drug Metabolism and Disposition 47(4):419-423

Baruscotti M, Barbuti A, Bucchi A (2010) The cardiac pacemaker current. Journal of molecular and cellular cardiology 48(1):55-64

Benet LZ, Zia-Amirhosseini P (1995) Basic principles of pharmacokinetics. Toxicologic pathology 23(2):115123

Berezhkovskiy LM (2011) The corrected traditional equations for calculation of hepatic clearance that account for the difference in drug ionization in extracellular and intracellular tissue water and the corresponding corrected PBPK equation. Journal of pharmaceutical sciences 100(3):1167-1183

Bil W, Zeilmaker M, Fragki S, et al. (2021) Risk assessment of per-and polyfluoroalkyl substance mixtures: A relative potency factor approach. Environmental Toxicology and Chemistry 40(3):859-870

Blanchette AD, Burnett SD, Grimm FA, Rusyn I, Chiu WA (2020) A Bayesian Method for Population-wide Cardiotoxicity Hazard and Risk Characterization Using an In Vitro Human Model. Toxicological Sciences 178(2):391-403

Blinova K, Dang Q, Millard D, et al. (2018) International multisite study of human-induced pluripotent stem cellderived cardiomyocytes for drug proarrhythmic potential assessment. Cell reports 24(13):3582-3592

Bosgra S, van der Voet H, Boon PE, Slob W (2009) An integrated probabilistic framework for cumulative risk assessment of common mechanism chemicals in food: an example with organophosphorus pesticides. Regulatory toxicology and pharmacology 54(2):124-133 
Bowman C, Benet L (2018) An examination of protein binding and protein-facilitated uptake relating to in vitroin vivo extrapolation. European Journal of Pharmaceutical Sciences 123:502-514

Brandon EF, Raap CD, Meijerman I, et al. (2003) An update on in vitro test methods in human hepatic drug biotransformation research: pros and cons. Toxicology and applied pharmacology 189(3):233-246

Britton OJ, Abi-Gerges N, Page G, et al. (2017) Quantitative comparison of effects of dofetilide, sotalol, quinidine, and verapamil between human ex vivo trabeculae and in silico ventricular models incorporating interindividual action potential variability. Frontiers in physiology 8:597

Brunner M, Langer O (2006) Microdialysis versus other techniques for the clinical assessment of in vivo tissue drug distribution. The AAPS journal 8(2):E263-E271

Burczynski F, Wang G, Elmadhoun B, et al. (2001) Hepatocyte [3H]-palmitate uptake: effect of albumin surface charge modification. Canadian journal of physiology and pharmacology 79(10):868-875

Burnett SD, Blanchette AD, Chiu WA, Rusyn I (2021) Human induced pluripotent stem cell (iPSC)-derived cardiomyocytes as an in vitro model in toxicology: strengths and weaknesses for hazard identification and risk characterization. Expert Opinion on Drug Metabolism \& Toxicology:1-16

Burnett SD, Blanchette AD, Grimm FA, et al. (2019) Population-based toxicity screening in human induced pluripotent stem cell-derived cardiomyocytes. Toxicology and applied pharmacology 381:114711

Campbell SD, Gadel S, Friedel C, et al. (2015) Influence of HIV antiretrovirals on methadone N-demethylation and transport. Biochemical pharmacology 95(2):115-125

Chaudhari U, Nemade H, Wagh V, et al. (2016) Identification of genomic biomarkers for anthracycline-induced cardiotoxicity in human iPSC-derived cardiomyocytes: an in vitro repeated exposure toxicity approach for safety assessment. Archives of toxicology 90(11):2763-2777

Chen Y, Liu L, Nguyen K, Fretland AJ (2011) Utility of intersystem extrapolation factors in early reaction phenotyping and the quantitative extrapolation of human liver microsomal intrinsic clearance using recombinant cytochromes P450. Drug metabolism and disposition 39(3):373-382

Chen L, Peijnenburg A, de Haan L, Rietjens IM (2019) Prediction of in vivo genotoxicity of lasiocarpine and riddelliine in rat liver using a combined in vitro-physiologically based kinetic modelling-facilitated reverse dosimetry approach. Archives of toxicology 93(8):2385-2395

Choi G-W, Lee Y-B, Cho H-Y (2019) Interpretation of non-clinical data for prediction of human pharmacokinetic parameters: in vitro-in vivo extrapolation and allometric scaling. Pharmaceutics 11(4):168

Clewell HJ, Tan YM, Campbell JL, Andersen ME (2008) Quantitative interpretation of human biomonitoring data. Toxicology and applied pharmacology 231(1):122-133

Comenges JZ, Joossens E, Benito JS, et al. (2017) Theoretical and mathematical foundation of the virtual cell based assay-a review. Toxicology in Vitro 45:209-221

Covington TR, Gentry PR, Van Landingham CB, et al. (2007) The use of Markov chain Monte Carlo uncertainty analysis to support a Public Health Goal for perchloroethylene. Regulatory Toxicology and Pharmacology 47(1):1-18

Crewe H, Barter Z, Rowland Yeo K, Rostami-Hodjegan A (2011) Are there differences in the catalytic activity per unit enzyme of recombinantly expressed and human liver microsomal cytochrome P450 2C9? A systematic investigation into inter-system extrapolation factors. Biopharmaceutics \& drug disposition 32(6):303-318 
Crumb Jr WJ, Vicente J, Johannesen L, Strauss DG (2016) An evaluation of 30 clinical drugs against the comprehensive in vitro proarrhythmia assay (CiPA) proposed ion channel panel. Journal of pharmacological and toxicological methods 81:251-262

Daga PR, Bolger MB, Haworth IS, et al. (2018) Physiologically based pharmacokinetic modeling in lead optimization. 1. Evaluation and adaptation of GastroPlus to predict bioavailability of Medchem series. Molecular pharmaceutics 15(3):821-830

Danik S, Cabo C, Chiello C, et al. (2002) Correlation of repolarization of ventricular monophasic action potential with ECG in the murine heart. American Journal of Physiology-Heart and Circulatory Physiology 283(1):H372-H381

Deb PK, Al-Attraqchi O, Prasad MR, Tekade RK (2018) Protein and tissue binding: implication on pharmacokinetic parameters Dosage Form Design Considerations. Elsevier, p 371-399

DeJongh J, Verhaar HJ, Hermens JL (1997) A quantitative property-property relationship (QPPR) approach to estimate in vitro tissue-blood partition coefficients of organic chemicals in rats and humans. Archives of Toxicology 72(1):17-25

Dubbelboer IR, Lilienberg E, Sjögren E, Lennernäs H (2017) A model-based approach to assessing the importance of intracellular binding sites in doxorubicin disposition. Molecular pharmaceutics 14(3):686-698

Eap CB, Buclin T, Baumann P (2002) Interindividual variability of the clinical pharmacokinetics of methadone. Clinical pharmacokinetics 41(14):1153-1193

Egashira T, Yuasa S, Suzuki T, et al. (2012) Disease characterization using LQTS-specific induced pluripotent stem cells. Cardiovascular research 95(4):419-429

European Food Safety Authority (EFSA) (2013). International frameworks dealing with human risk assessment of combined exposure to multiple chemicals. Efsa Journal, 11(7), 3313.

European Food Safety Authority (EFSA) (2019). Guidance on harmonised methodologies for human health, animal health and ecological risk assessment of combined exposure to multiple chemicals. Efsa journal, 17(3).

Fagerholm U (2007) Prediction of human pharmacokinetics - evaluation of methods for prediction of hepatic metabolic clearance. Journal of pharmacy and pharmacology 59(6):803-828

Fischer FC, Henneberger L, König M, et al. (2017) Modeling exposure in the Tox21 in vitro bioassays. Chemical research in toxicology 30(5):1197-1208

Fisher C, Simeon S, Jamei M, et al. (2019) VIVD: virtual in vitro distribution model for the mechanistic prediction of intracellular concentrations of chemicals in in vitro toxicity assays. Toxicology in Vitro 58:42-50

Fisher JW, Campbell Jr JL, Lin Z (2020) Metabolism and physiologically based pharmacokinetic models Physiologically Based Pharmacokinetic (PBPK) Modeling. Elsevier, p 161-173

Foster DJR (2001) An examination of the metabolism and pharmacokinetics of methadone with respect to stereoselectivity/David JR Foster.

Gao H, Yao L, Mathieu HW, et al. (2008) In silico modeling of nonspecific binding to human liver microsomes. Drug metabolism and disposition 36(10):2130-2135

Giacomini KM, Huang S-M, Tweedie DJ, et al. (2010) Membrane transporters in drug development. Nature reviews Drug discovery 9(3):215 
Gilbert-Sandoval I, Wesseling S, Rietjens IMCM (2020) Predicting the Acute Liver Toxicity of Aflatoxin B1 in Rats and Humans by an In Vitro-In Silico Testing Strategy. Molecular nutrition \& food research 64(13):2000063

Clausen J, Bickel M (1993) Prediction of drug distribution in distribution dialysis and in vivo from binding to tissues and blood. Journal of pharmaceutical sciences 82(4):345-349

Glue P, Cape G, Tunnicliff D, et al. (2016) Ascending single-dose, double-blind, placebo-controlled safety study of noribogaine in opioid-dependent patients. Clinical pharmacology in drug development 5(6):460-468

Glue P, Lockhart M, Lam F, et al. (2015) Ascending-dose study of noribogaine in healthy volunteers: Pharmacokinetics, pharmacodynamics, safety, and tolerability. The Journal of Clinical Pharmacology 55(2):189-194

Grimm FA, Blanchette A, House JS, et al. (2018) A human population-based organotypic in vitro model for cardiotoxicity screening. Altex 35(4):441

Groothuis FA, Heringa MB, Nicol B, et al. (2015) Dose metric considerations in in vitro assays to improve quantitative in vitro-in vivo dose extrapolations. Toxicology 332:30-40

Guo L, Qian J-Y, Abrams R, et al. (2011) The electrophysiological effects of cardiac glycosides in human iPSCderived cardiomyocytes and in guinea pig isolated hearts. Cellular Physiology and Biochemistry 27(5):453-462

Haddad S, Nong A (2020) Physiologically based pharmacokinetic model: excretion via urine, feces, and breath Physiologically Based Pharmacokinetic (PBPK) Modeling. Elsevier, p 175-209

Hallifax D, Houston JB (2012) Evaluation of hepatic clearance prediction using in vitro data: emphasis on fraction unbound in plasma and drug ionisation using a database of 107 drugs. Journal of pharmaceutical sciences 101(8):2645-2652

Hamon J, Renner M, Jamei M, et al. (2015) Quantitative in vitro to in vivo extrapolation of tissues toxicity. Toxicology in Vitro 30(1):203-216

Heuberger J, Schmidt S, Derendorf H (2013) When is protein binding important? Journal of pharmaceutical sciences 102(9):3458-3467

Himmel HM (2013) Drug-induced functional cardiotoxicity screening in stem cell-derived human and mouse cardiomyocytes: effects of reference compounds. Journal of pharmacological and toxicological methods 68(1):97-111

Hirano M, Maeda K, Matsushima S, et al. (2005) Involvement of BCRP (ABCG2) in the biliary excretion of pitavastatin. Molecular pharmacology 68(3):800-807

Hoekstra M, Mummery CL, Wilde AA, et al. (2012) Induced pluripotent stem cell derived cardiomyocytes as models for cardiac arrhythmias. Frontiers in physiology 3:346

Hoelen DW, Spiering W, Valk GD (2009) Long-QT syndrome induced by the antiaddiction drug ibogaine. New England journal of medicine 360(3):308-309

Hondeghem L, De Clerck F (2012) Preclinical cardiovascular safety evaluations of biologics. BioDrugs 26(5):275-282

Hou T, Zhang W, Xia K, et al. (2004) ADME evaluation in drug discovery. 5. Correlation of Caco-2 permeation with simple molecular properties. Journal of chemical information and computer sciences 44(5):15851600 
Howard ML, Hill JJ, Galluppi GR, McLean MA (2010) Plasma protein binding in drug discovery and development. Combinatorial chemistry \& high throughput screening 13(2):170-187

Huang CL-H (2017) Murine electrophysiological models of cardiac arrhythmogenesis. Physiological reviews 97(1):283-409

Huang W, Isoherranen N (2018) Development of a dynamic physiologically based mechanistic kidney model to predict renal clearance. CPT: pharmacometrics \& systems pharmacology 7(9):593-602

Ikeuchi T, Espulgar W, Shimizu E, et al. (2015) Optical microscopy imaging for the diagnosis of the pharmacological reaction of mouse embryonic stem cell-derived cardiomyocytes (mESC-CMs). Analyst 140(19):6500-6507

International Programme on Chemical Safety (IPCS) (2005). Chemical-specific adjustment factors for interspecies differences and human variability: guidance document for use of data in dose/ concentrationresponse assessment. WHO, Geneva. https://apps.who.int/iris/bitstream/handle/10665/43294/9241546786_eng.pdf?sequence=1\&isAllowed= $\mathrm{y}$

Itzhaki I, Maizels L, Huber I, et al. (2011) Modelling the long QT syndrome with induced pluripotent stem cells. Nature 471(7337):225-229

Kaese S, Verheule S (2012) Cardiac electrophysiology in mice: a matter of size. Frontiers in physiology 3:345

Kalvass JC, Tess DA, Giragossian C, et al. (2001) Influence of microsomal concentration on apparent intrinsic clearance: implications for scaling in vitro data. Drug metabolism and disposition 29(10):1332-1336

Karakikes I, Ameen M, Termglinchan V, Wu JC (2015) Human induced pluripotent stem cell-derived cardiomyocytes: insights into molecular, cellular, and functional phenotypes. Circulation research 117(1):80-88

Kasteel EE, Westerink RH (2021) Refining in vitro and in silico neurotoxicity approaches by accounting for interspecies and interindividual differences in toxicodynamics. Expert Opinion on Drug Metabolism \& Toxicology:1-11

Ke AB, Nallani SC, Zhao P, et al. (2014) Expansion of a PBPK model to predict disposition in pregnant women of drugs cleared via multiple CYP enzymes, including CYP2B6, CYP2C9 and CYP2C19. British journal of clinical pharmacology 77(3):554-570

Kharasch ED, Hoffer C, Whittington D, Sheffels P (2004) Role of hepatic and intestinal cytochrome P450 3A and 2B6 in the metabolism, disposition, and miotic effects of methadone. Clinical Pharmacology \& Therapeutics 76(3):250-269

Kharasch ED, Walker A, Whittington D, et al. (2009) Methadone metabolism and clearance are induced by nelfinavir despite inhibition of cytochrome P4503A (CYP3A) activity. Drug and alcohol dependence 101(3):158-168

Kilford PJ, Gertz M, Houston JB, Galetin A (2008) Hepatocellular binding of drugs: correction for unbound fraction in hepatocyte incubations using microsomal binding or drug lipophilicity data. Drug Metabolism and Disposition 36(7):1194-1197

Koenig X, Kovar M, Boehm S, et al. (2014) Anti-addiction drug ibogaine inhibits hERG channels: a cardiac arrhythmia risk. Addiction biology 19(2):237-239

Kotsiou A, Tesseromatis C (2011) Protein binding of drugs. Oral Bioavailability: Basic Principles, Advanced Concepts, and Applications:145-166 
Kratz JM, Grienke U, Scheel O, et al. (2017) Natural products modulating the hERG channel: heartaches and hope. Natural product reports 34(8):957-980

Kraushaar U, Guenther E (2019) Assay procedures for compound testing of hiPSC-derived cardiomyocytes using multiwell microelectrode arrays Cell-Based Assays Using iPSCs for Drug Development and Testing. Springer, p 197-208

Krishna S, Berridge B, Kleinstreuer N (2020) High-Throughput Screening to Identify Chemical Cardiotoxic Potential. Chemical Research in Toxicology

Kumar V, Wahlstrom JL, Rock DA, et al. (2006) CYP2C9 inhibition: impact of probe selection and pharmacogenetics on in vitro inhibition profiles. Drug metabolism and disposition 34(12):1966-1975

Lee S-G, Kim J, Oh M-S, et al. (2021) Development and validation of dual-cardiotoxicity evaluation method based on analysis of field potential and contractile force of human iPSC-derived cardiomyocytes/multielectrode assay platform. Biochemical and Biophysical Research Communications 555:67-73

Li H, Yuan H, Middleton A, et al. (2021) Next generation risk assessment (NGRA): Bridging in vitro points-ofdeparture to human safety assessment using physiologically-based kinetic (PBK) modelling-A case study of doxorubicin with dose metrics considerations. Toxicology in Vitro:105171

Lipscomb JC, Poet TS (2008) In vitro measurements of metabolism for application in pharmacokinetic modeling. Pharmacology \& therapeutics 118(1):82-103

Lohse M, Engelhardt S, Danner S, Böhm M (1996) Mechanisms of $\beta$-adrenergic receptor desensitization: from molecular biology to heart failure. Basic research in cardiology 91(1):29-34

Louisse J, de Jong E, van de Sandt JJ, et al. (2010) The use of in vitro toxicity data and physiologically based kinetic modeling to predict dose-response curves for in vivo developmental toxicity of glycol ethers in rat and man. Toxicological Sciences 118(2):470-484

Louisse J, Beekmann K, Rietjens IMCM (2017) Use of physiologically based kinetic modeling-based reverse dosimetry to predict in vivo toxicity from in vitro data. Chem Res Toxicol 30:114-125

Ma J, Guo L, Fiene SJ, et al. (2011) High purity human-induced pluripotent stem cell-derived cardiomyocytes: electrophysiological properties of action potentials and ionic currents. American Journal of PhysiologyHeart and Circulatory Physiology 301(5):H2006-H2017

Mallick P, Moreau M, Song G, et al. (2020) Development and application of a life-stage physiologically based pharmacokinetic (PBPK) model to the assessment of internal dose of pyrethroids in humans. Toxicological Sciences 173(1):86-99

Martin RL, McDermott JS, Salmen HJ, et al. (2004) The utility of hERG and repolarization assays in evaluating delayed cardiac repolarization: influence of multi-channel block. Journal of cardiovascular pharmacology 43(3):369-379

Matsushima S, Maeda K, Kondo C, et al. (2005) Identification of the hepatic efflux transporters of organic anions using double-transfected Madin-Darby canine kidney II cells expressing human organic aniontransporting polypeptide 1B1 (OATP1B1)/multidrug resistance-associated protein 2, OATP1B1/multidrug resistance 1, and OATP1B1/breast cancer resistance protein. Journal of Pharmacology and Experimental Therapeutics 314(3):1059-1067

Mikkelsen CR, Jornil JR, Andersen LV, et al. (2018) Utilizing postmortem drug concentrations in mechanistic modeling and simulation of cardiac effects: a proof of concept study with methadone. Toxicology mechanisms and methods 28(8):555-562 
Millard D, Dang Q, Shi H, et al. (2018) Cross-site reliability of human induced pluripotent stem cell-derived cardiomyocyte based safety assays using microelectrode arrays: results from a blinded CiPA pilot study. Toxicological Sciences 164(2):550-562

Moroy G, Martiny VY, Vayer P, et al. (2012) Toward in silico structure-based ADMET prediction in drug discovery. Drug discovery today 17(1-2):44-55

Mukherjee D, Royce SG, Alexander JA, et al. (2014) Physiologically-based toxicokinetic modeling of zearalenone and its metabolites: application to the Jersey girl study. PLoS One 9(12):e113632

Mulder P, de Korte T, Dragicevic E, et al. (2018) Predicting cardiac safety using human induced pluripotent stem cell-derived cardiomyocytes combined with multi-electrode array (MEA) technology: A conference report. Journal of pharmacological and toxicological methods 91:36-42

Nerbonne JM (2004) Studying cardiac arrhythmias in the mouse - a reasonable model for probing mechanisms? Trends in cardiovascular medicine 14(3):83-93

Nichols J, Fay K, Bernhard MJ, et al. (2018) Reliability of in vitro methods used to measure intrinsic clearance of hydrophobic organic chemicals by rainbow trout: Results of an international ring trial. Toxicological sciences 164(2):563-575

Ning J, Rietjens IMCM, Strikwold M (2019) Integrating physiologically based kinetic (PBK) and Monte Carlo modelling to predict inter-individual and inter-ethnic variation in bioactivation and liver toxicity of lasiocarpine. Archives of toxicology 93(10):2943-2960

Nong A, Taylor MD, Clewell III HJ, et al. (2009) Manganese tissue dosimetry in rats and monkeys: accounting for dietary and inhaled Mn with physiologically based pharmacokinetic modeling. Toxicological sciences 108(1):22-34

Noorlander A, Fabian E, van Ravenzwaay B, Rietjens IMCM (2021a) Novel testing strategy for prediction of rat biliary excretion of intravenously administered estradiol-17 $\beta$ glucuronide. Archives of Toxicology 95(1):91-102

Noorlander A, Wesseling S, Rietjens IMCM, van Ravenzwaay B (2021b) Incorporating renal excretion via the OCT2 transporter in physiologically based kinetic modelling to predict in vivo kinetics of mepiquat in rat. Toxicology Letters 343:34-43

Nunes SS, Miklas JW, Liu J, et al. (2013) Biowire: a platform for maturation of human pluripotent stem cellderived cardiomyocytes. Nature methods 10(8):781-787

Obach RS (1999) Prediction of human clearance of twenty-nine drugs from hepatic microsomal intrinsic clearance data: an examination of in vitro half-life approach and nonspecific binding to microsomes. Drug Metabolism and Disposition 27(11):1350-1359

Obach RS, Pablo J, Mash DC (1998) Cytochrome P4502D6 catalyzes the O-demethylation of the psychoactive alkaloid ibogaine to 12-hydroxyibogamine. Drug metabolism and disposition 26(8):764-768

Omwenga I, Zhao S, Kanja L, et al. (2021) Prediction of dose-dependent in vivo acetylcholinesterase inhibition by profenofos in rats and humans using physiologically based kinetic (PBK) modeling-facilitated reverse dosimetry. Archives of Toxicology:1-15

Ooka M, Lynch C, Xia M (2020) Application of In Vitro Metabolism Activation in High-Throughput Screening. International Journal of Molecular Sciences 21(21):8182 .

Organisation for Economic Co-operation and Development (OECD) (2021) Guidance document on the characterisation, validation and reporting of Physiologically Based Kinetic (PBK) models for regulatory 
purposes $\quad \underline{\text { https://ec.europa.eu/jrc/en/science-update/new-guidance-using-computational-models- }}$ chemical-risk-assessment

Ovics P, Regev D, Baskin P, et al. (2020) Drug Development and the Use of Induced Pluripotent Stem CellDerived Cardiomyocytes for Disease Modeling and Drug Toxicity Screening. International Journal of Molecular Sciences 21(19):7320

Pang L, Sager P, Yang X, et al. (2019) Workshop report: FDA workshop on improving cardiotoxicity assessment with human-relevant platforms. Circulation research 125(9):855-867

Panopoulos AD, D'Antonio M, Benaglio P, et al. (2017) iPSCORE: a resource of 222 iPSC lines enabling functional characterization of genetic variation across a variety of cell types. Stem cell reports 8(4):10861100

Patel AK, Celiz AD, Rajamohan D, et al. (2015) A defined synthetic substrate for serum-free culture of human stem cell derived cardiomyocytes with improved functional maturity identified using combinatorial materials microarrays. Biomaterials 61:257-265

Poulin P, Haddad S (2011) Microsome composition-based model as a mechanistic tool to predict nonspecific binding of drugs in liver microsomes. Journal of pharmaceutical sciences 100(10):4501-4517

Poulin P, Kenny JR, Hop CE, Haddad S (2012) In vitro-in vivo extrapolation of clearance: Modeling hepatic metabolic clearance of highly bound drugs and comparative assessment with existing calculation methods. Journal of pharmaceutical sciences 101(2):838-851

Poulin P, Theil F-P (2002) Prediction of pharmacokinetics prior to in vivo studies. II. Generic physiologically based pharmacokinetic models of drug disposition. Journal of pharmaceutical sciences 91(5):1358-1370

Pourrier M, Fedida D (2020) The emergence of human induced pluripotent stem cell-derived cardiomyocytes (hiPSC-CMs) as a platform to model arrhythmogenic diseases. International journal of molecular sciences 21(2):657

Proctor N, Tucker G, Rostami-Hodjegan A (2004) Predicting drug clearance from recombinantly expressed CYPs: intersystem extrapolation factors. Xenobiotica 34(2):151-178

Punt A, Pinckaers N, Peijnenburg A, Louisse J (2020) Development of a Web-Based Toolbox to Support Quantitative In-Vitro-to-In-Vivo Extrapolations (QIVIVE) within Nonanimal Testing Strategies. Chemical Research in Toxicology

Quindroit P, Beaudouin R, Brochot C (2019) Estimating the cumulative human exposures to pyrethroids by combined multi-route PBPK models: application to the French population. Toxicology letters 312:125138

Rajamohan D, Matsa E, Kalra S, et al. (2013) Current status of drug screening and disease modelling in human pluripotent stem cells. Bioessays 35(3):281-298

Riccardi K, Ryu S, Lin J, et al. (2018) Comparison of species and cell-type differences in fraction unbound of liver tissues, hepatocytes, and cell lines. Drug Metabolism and Disposition 46(4):415-421

Rubi L, Eckert D, Boehm S, et al. (2017) Anti-addiction drug ibogaine prolongs the action potential in human induced pluripotent stem cell-derived cardiomyocytes. Cardiovascular toxicology 17(2):215-218

Safe SH (1998) Hazard and risk assessment of chemical mixtures using the toxic equivalency factor approach. Environmental health perspectives 106(suppl 4):1051-1058

Sala L, Ward-van Oostwaard D, Tertoolen LG, Mummery CL, Bellin M (2017) Electrophysiological analysis of human pluripotent stem cell-derived cardiomyocytes (hPSC-CMs) using multi-electrode arrays (MEAs). JoVE (Journal of Visualized Experiments)(123):e55587 
Sanguinetti MC, Jiang C, Curran ME, Keating MT (1995) A mechanistic link between an inherited and an acquird cardiac arrthytmia: HERG encodes the IKr potassium channel. Cell 81(2):299-307

Shinozawa T, Nakamura K, Shoji M, et al. (2017) Recapitulation of clinical individual susceptibility to druginduced QT prolongation in healthy subjects using iPSC-derived cardiomyocytes. Stem Cell Reports $8(2): 226-234$

Sipes NS, Wambaugh JF, Pearce R, et al. (2017) An intuitive approach for predicting potential human health risk with the Tox21 10k library. Environmental science \& technology 51(18):10786-10796

Sirenko O, Grimm FA, Ryan KR, et al. (2017) In vitro cardiotoxicity assessment of environmental chemicals using an organotypic human induced pluripotent stem cell-derived model. Toxicology and applied pharmacology 322:60-74

Soldatow VY, LeCluyse EL, Griffith LG, Rusyn I (2013) In vitro models for liver toxicity testing. Toxicology research 2(1):23-39

Stresser DM, Blanchard AP, Turner SD, et al. (2000) Substrate-dependent modulation of CYP3A4 catalytic activity: analysis of 27 test compounds with four fluorometric substrates. Drug Metabolism and Disposition 28(12):1440-1448

Strikwold M, Spenkelink B, de Haan LH, et al. (2017) Integrating in vitro data and physiologically based kinetic (PBK) modelling to assess the in vivo potential developmental toxicity of a series of phenols. Archives of toxicology 91(5):2119-2133

Strikwold M, Spenkelink B, Woutersen RA, et al. (2013) Combining in vitro embryotoxicity data with physiologically based kinetic (PBK) modelling to define in vivo dose-response curves for developmental toxicity of phenol in rat and human. Archives of toxicology 87(9):1709-1723

Sun D, Lennernas H, Welage LS, et al. (2002) Comparison of human duodenum and Caco-2 gene expression profiles for 12,000 gene sequences tags and correlation with permeability of 26 drugs. Pharmaceutical research 19(10):1400-1416

Sun N, Yazawa M, Liu J, et al. (2012) Patient-specific induced pluripotent stem cells as a model for familial dilated cardiomyopathy. Science translational medicine 4(130):130ra47-130ra47

Sweeney LM, Gearhart JM (2020) Examples of physiologically based pharmacokinetic modeling applied to risk assessment Physiologically Based Pharmacokinetic (PBPK) Modeling. Elsevier, p 281-299

T'jollyn H, Snoeys J, Colin P, et al. (2015) Physiology-based IVIVE predictions of tramadol from in vitro metabolism data. Pharmaceutical research 32(1):260-274

Tertoolen L, Braam S, Van Meer B, et al. (2018) Interpretation of field potentials measured on a multi electrode array in pharmacological toxicity screening on primary and human pluripotent stem cell-derived cardiomyocytes. Biochemical and biophysical research communications 497(4):1135-1141

Thomas D, Karle C, Kiehn J (2006) The cardiac hERG/IKr potassium channel as pharmacological target: structure, function, regulation, and clinical applications. Current pharmaceutical design 12(18):2271-2283

Umehara K-i, Huth F, Gu H, et al. (2017) Estimation of fractions metabolized by hepatic CYP enzymes using a concept of inter-system extrapolation factors (ISEFs)-a comparison with the chemical inhibition method. Drug metabolism and personalized therapy 32(4):191-200

U.S. Environmental Protection Agency (EPA) (2000) Supplementary Guidance for Conducting Health Risk Assessment of Chemical Mixtures. ORD.NECA. Washington, DC. EPA/630/R-00/002.

Uzun AU, Mannhardt I, Breckwoldt K, et al. (2016) Ca2+-currents in human induced pluripotent stem cell-derived cardiomyocytes effects of two different culture conditions. Frontiers in pharmacology 7:300 
Vallianatou T, Lambrinidis G, Tsantili-Kakoulidou A (2013) In silico prediction of human serum albumin binding for drug leads. Expert opinion on drug discovery 8(5):583-595

Van Brantegem P, Deferm N, Qi B, et al. (2019) Vesicle-and Hepatocyte-Based Assays for Identification of Drug Candidates Inhibiting BSEP Function Experimental Cholestasis Research. Springer, p 55-73

van Ede KI, van Duursen MB, van den Berg M (2016) Evaluation of relative effect potencies (REPs) for dioxinlike compounds to derive systemic or human-specific TEFs to improve human risk assessment. Archives of toxicology 90(6):1293-1305

van Liempd S, Morrison D, Sysmans L, et al. (2011) Development and validation of a higher-throughput equilibrium dialysis assay for plasma protein binding. JALA: Journal of the Association for Laboratory Automation 16(1):56-67

Wageningen Food Safety Research (WFSR) (2020). QIVIVE tools-Partition coefficients. [Online].

Wang C, Williams NS (2013) A mass balance approach for calculation of recovery and binding enables the use of ultrafiltration as a rapid method for measurement of plasma protein binding for even highly lipophilic compounds. Journal of pharmaceutical and biomedical analysis 75:112-117

Wetmore BA, Wambaugh JF, Ferguson SS, et al. (2012) Integration of dosimetry, exposure, and high-throughput screening data in chemical toxicity assessment. Toxicological Sciences 125(1):157-174

Wetmore BA, Allen B, Clewell III HJ, et al. (2014) Incorporating population variability and susceptible subpopulations into dosimetry for high-throughput toxicity testing. Toxicological Sciences 142(1):210224

Wetmore BA, Wambaugh JF, Allen B, et al. (2015) Incorporating high-throughput exposure predictions with dosimetry-adjusted in vitro bioactivity to inform chemical toxicity testing. Toxicological Sciences 148(1):121-136

Williams PA, Cosme J, Ward A, et al. (2003) Crystal structure of human cytochrome P450 2C9 with bound warfarin. Nature 424(6947):464-468

World Health Organization (WHO) (2016) Toxicity equivalence factors for marine biotoxins associated with bivalve molluscs.

Worley RR, Fisher J (2015) Application of physiologically-based pharmacokinetic modeling to explore the role of kidney transporters in renal reabsorption of perfluorooctanoic acid in the rat. Toxicology and applied pharmacology 289(3):428-441

Worth AP, Louisse J, Macko P, et al. (2017) Virtual Cell Based Assay simulations of intra-mitochondrial concentrations in hepatocytes and cardiomyocytes. Toxicology in Vitro 45:222-232

Xu H, Guo W, Nerbonne JM (1999) Four kinetically distinct depolarization-activated K+ currents in adult mouse ventricular myocytes. The Journal of general physiology 113(5):661-678

Yang F, Tong X, McCarver DG, et al. (2006) Population-based analysis of methadone distribution and metabolism using an age-dependent physiologically based pharmacokinetic model. Journal of pharmacokinetics and pharmacodynamics 33(4):485-518

Yang X, Rodriguez M, Pabon L, et al. (2014) Tri-iodo-l-thyronine promotes the maturation of human cardiomyocytes-derived from induced pluripotent stem cells. Journal of molecular and cellular cardiology 72:296-304 
Yang X, Wu H, Mehta D, et al. (2019) Ontogeny equations with probability distributions for anthropomorphic measurements in preterm and term neonates and infants for use in a PBPK model. Computational Toxicology 11:101-117

Ye M, Nagar S, Korzekwa K (2016) A physiologically based pharmacokinetic model to predict the pharmacokinetics of highly protein-bound drugs and the impact of errors in plasma protein binding. Biopharmaceutics \& drug disposition 37(3):123-141

Ye Z, Zetterberg C, Gao H (2017) Automation of plasma protein binding assay using rapid equilibrium dialysis device and Tecan workstation. Journal of pharmaceutical and biomedical analysis 140:210-214

Yoon M (2020) Physiologically based pharmacokinetic models to support modernized chemical safety assessment Physiologically Based Pharmacokinetic (PBPK) Modeling. Elsevier, p 301-321

Youdim KA, Zayed A, Dickins M, et al. (2008) Application of CYP3A4 in vitro data to predict clinical drugdrug interactions; predictions of compounds as objects of interaction. British journal of clinical pharmacology 65(5):680-692

Zeise L, Bois FY, Chiu WA, et al. (2013) Addressing human variability in next-generation human health risk assessments of environmental chemicals. Environmental health perspectives 121(1):23-31

Zhang D, Luo G, Ding X, Lu C (2012) Preclinical experimental models of drug metabolism and disposition in drug discovery and development. Acta Pharmaceutica Sinica B 2(6):549-561

Zhang M, van Ravenzwaay B, Fabian E, et al. (2018) Towards a generic physiologically based kinetic model to predict in vivo uterotrophic responses in rats by reverse dosimetry of in vitro estrogenicity data. Archives of toxicology 92(3):1075-1088

Zhang M, van Ravenzwaay B, Rietjens IMCM (2020) Development of a generic physiologically based kinetic model to predict in vivo uterotrophic responses induced by estrogenic chemicals in rats based on in vitro bioassays. Toxicological Sciences 173(1):19-31

Zhang X, Tsang AM, Okino MS, et al. (2007) A physiologically based pharmacokinetic/pharmacodynamic model for carbofuran in Sprague-Dawley rats using the exposure-related dose estimating model. Toxicological Sciences 100(2):345-359

Zhao S, Kamelia L, Boonpawa R, et al. (2019) Physiologically based kinetic modeling-facilitated reverse dosimetry to predict in vivo red blood cell acetylcholinesterase inhibition following exposure to chlorpyrifos in the Caucasian and Chinese population. Toxicological sciences 171(1):69-83

Zwartsen A, de Korte T, Nacken P, et al. (2019) Cardiotoxicity screening of illicit drugs and new psychoactive substances (NPS) in human iPSC-derived cardiomyocytes using microelectrode array (MEA) recordings. Journal of molecular and cellular cardiology 136:102-112 


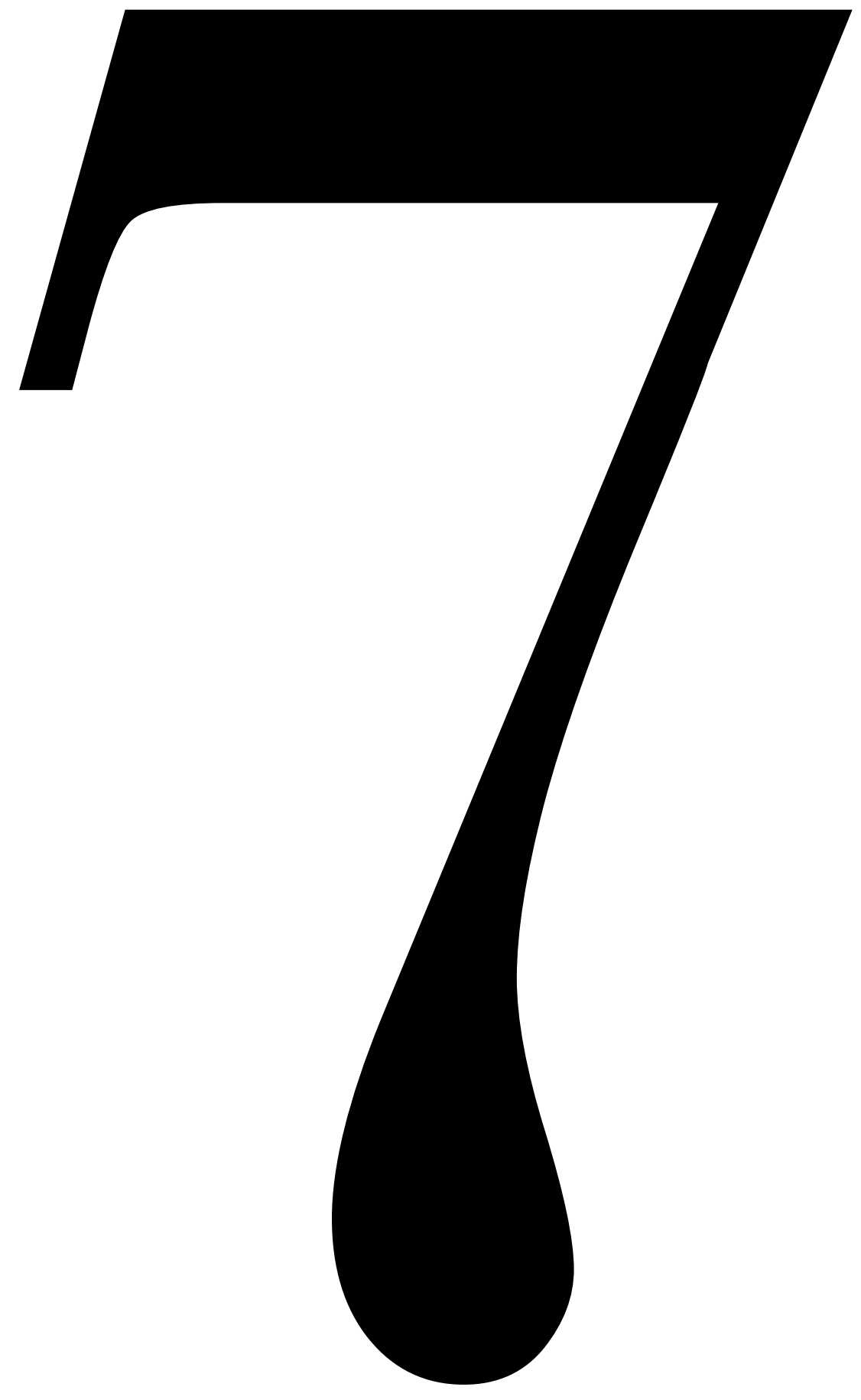


Chapter 7

Summary 


\section{Summary}

The inter-species differences between animals and human and ethical and financial concerns related to studies with experimental animals provide a strong motivation for a paradigm shift in toxicity testing of chemicals. This initiated, as also shown in the current thesis, the development of new approach methodologies (NAMs) that integrate in vitro and in silico methods, to replace animal or even human studies. An example of such a NAM is the quantitative in vitro to in vivo extrapolation (QIVIVE) approach, also so-called PBK modelingbased reverse dosimetry. In this approach, as a starting point, in vitro data can be used to be translated using PBK modeling-facilitated reverse dosimetry to predict dose-response curves for the in vivo situation. This approach has already adequately predicted the in vivo toxicity for various toxicity endpoints in recent years. So far these proofs-of-principle did not relate to cardiotoxicity and mainly related to toxicity in experimental animals. The present thesis aimed to provide proofs-of-principle for using PBK modeling-based reverse dosimetry of in vitro data for the prediction of cardiotoxicity in humans, thereby providing a novel testing strategy for cardiac safety studies. Methadone and ibogaine, two anti-addiction drugs with known in vivo cardiotoxicity, were selected as model compounds. The developed QIVIVE approach can contribute to non-animal based NAMs for risk assessment and safety evaluation of chemicals.

Chapter 1 introduced background information on alternative testing strategies and the aim of the present thesis. It also provided information on cardiotoxicity, on the toxicokinetic and toxicodynamic profiles of the two model compounds and their metabolites and on the main approaches applied in the present thesis, including two in vitro cardiotoxicity assays, PBK modelling-based reverse dosimetry and Monte Carlo simulation.

Chapter 2 evaluated the applicability domains of two stem cell-based in vitro models for cardiotoxicity screening of chemicals, namely the mouse embryonic stem cell-derived cardiomyocyte (mESC-CM) model that uses beating arrest as a readout and the human induced pluripotent stem cell-derived cardiomyocyte (hiPSC-CM) multi-electrode array (MEA) assay that uses multiple electrophysiological parameters as readouts. The cardiotoxicity of eleven model compounds, including hERG channel blockers, sodium channel blockers, calcium channel blockers, $\mathrm{Na}^{+} / \mathrm{K}^{+}$ATPase inhibitors and a $\beta$-adrenergic receptor agonist, were detected in the two models. The results obtained from the two models were compared to one another and to in vivo cardiotoxicity data. Results showed that the $\mathrm{mESC}-\mathrm{CM}$ beating arrest assay was not responsive to hERG channel blockers and a $\mathrm{Na}^{+} / \mathrm{K}^{+}$ATPase inhibitor. Whereas the hiPSC- 
CM MEA assay was responsive to all model compounds and also resulted in in vitro effective concentrations that correlated well with in vivo concentrations associated with responses in the human electrocardiogram. Overall, the results indicated that the mESC-CM beating arrest assay could be used as the first step in a tiered approach for cardiotoxicity screening to detect hazards related to cardiotoxicity via effects on for example sodium and calcium ion channels. The hiPSC-CM MEA assay provided comprehensive cardiotoxicity data and thus could be used as a second tier to detect cardiotoxicity, and it may also serve as a basis for QIVIVE to predict human cardiotoxicity.

Chapter 3 demonstrated that the combination of the hiPSC-CM MEA assay and PBK modeling-based reverse dosimetry adequately predicted methadone-induced cardiotoxicity in human. The in vitro cardiotoxicity of methadone was detected using the prolongation of the field potential duration corrected for beat rate (FPDc) in the hiPSC-CM MEA assay. A human PBK model of methadone was developed using data obtained from in silico predictions, in vitro microsomal incubations and literature. The in vitro concentration-response curve of methadone was converted to an in vivo dose-response curve for QTc prolongation using different unbound fractions in human plasma given the large variation in protein plasma binding of methadone reported in in vivo human studies. Results revealed that the predictions using high and low fraction unbound values were well in line with data obtained from individual case studies and epidemiological population studies, respectively. Altogether, this chapter provided a proof-of-principle of using PBK modeling-based reverse dosimetry of in vitro cardiotoxicity data for the prediction of QTc prolongation in humans.

Chapter 4 further investigated the potential of the developed in vitro-in silico approach by predicting the influence of inter-individual and inter-ethnic kinetic variations on the sensitivity towards the cardiotoxicity of R- and S-methadone in the Caucasian and the Chinese population. Two sources of metabolic variation were used to quantify the inter-individual and inter-ethnic kinetic variability including individual human liver microsomes and recombinant cytochrome P450 isoforms (rCYPs). Data from these in vitro model systems were incorporated in the PBK models (and combined with Monte Carlo simulation when rCYPs data were used) to predict the interindividual and inter-ethnic variability in the kinetics of the methadone enantiomers. The results obtained showed that both approaches similarly predicted the inter-individual and inter-ethnic variations in the kinetics of the two enantiomers. The predicted blood kinetics obtained using rCYPs combined with Monte Carlo simulation were used to derive chemical specific adjustment factors (CSAFs), which were subsequently applied to define dose-response 
curves for the sensitive individuals within the populations by reverse dosimetry. Benchmark dose (BMD) analysis of the predicted dose-response curves provided BMDL values that were used to calculate the Margins of Safety (MOS) to evaluate the inter-ethnic differences in safety upon use of R- and S-methadone. Results obtained showed that the default uncertainty factor of 3.16 for kinetic differences appeared to adequately describe the interindividual differences in kinetics for R- and S-methadone-induced cardiotoxicity in the Caucasian and in the Chinese population. When considering the combined population, the default CSAF was still protective for R-methadone while for S-methadone the CSAF for kinetic differences increased to 4.5 and 4.7 for protection of the $95^{\text {th }}$ and $99^{\text {th }}$ percentile of the population, respectively. The results also revealed that Chinese individuals may be at higher risk towards the cardiotoxicity of methadone with the predicted BMDL and thus MOS values being 2-fold lower than those for Caucasians for both methadone enantiomers.

Chapter 5 investigated whether PBK modeling-based reverse dosimetry of in vitro data was able to adequately predict the human cardiotoxicity of the herbal alkaloid ibogaine and its metabolite noribogaine. The in vitro cardiotoxicity of ibogaine and noribogaine were quantified using the hiPSC-CM MEA assay. A human PBK model for ibogaine with a submodel for noribogaine was developed using parameters obtained from in silico approaches and literature, and biokinetic data obtained from Caco-2 transport studies and in vitro liver microsomal incubations. Because the unbound in vitro effective concentration of noribogaine was within the range of reported unbound internal blood concentrations of noribogaine after ingestion of clinically relevant doses of ibogaine, the cardiotoxicity of noribogaine was taken into account in the reverse dosimetry for the prediction of ibogaine-induced cardiotoxicity by using the toxic equivalency (TEQ) approach. Given that the oral administration of noribogaine is reported to be associated with QTc prolongation in humans, reverse dosimetry of in vitro cardiotoxicity of noribogaine was also performed to predict the in vivo noribogaine-induced QTc prolongation. BMD analysis of the predicted dose-response curves adequately predicted the in vivo cardiotoxicity upon oral exposure to ibogaine and noribogaine. Additionally, the relative contribution of ibogaine and noribogaine in ibogaine-induced cardiotoxicity was investigated by integrating the TEQ approach with the PBK model. The results thus obtained revealed that noribogaine is predicted to play a substantial role in the in vivo cardiotoxicity upon the oral administration of ibogaine.

Chapter 6 summarizes the results obtained in the thesis and discusses the applicability domains of the two in vitro cardiotoxicity assays, different considerations with regard to PBK 
modelling and reverse dosimetry and implications of these NAMs for risk assessment. Future perspectives outline the possibility of extending the developed PBK modeling-based reverse dosimetry approach to a broader application area in the field of risk assessment. Altogether, the present thesis provided proofs-of-principle for using PBK modeling-based reverse dosimetry of in vitro data for the prediction of cardiotoxicity in humans, demonstrating the potential applicability of this QIVIVE approach for a broader range of toxic endpoints and for the human situation, ultimately contributing to non-animal based NAMs for risk assessment and safety evaluation of chemicals. 


$$
\mathrm{A}
$$




\title{
Annex
}

\author{
Acknowledgments
}

About the author

List of publications

Overview of completed training activities 


\section{Acknowledgement}

I still remembered the feeling when I just started my PhD: I was about to embark on a sailing adventure with the anxiety and excitement about the future. It turned out that this voyage is not smooth, but luckily, I received countless guidance, encouragement and inspiration along my $\mathrm{PhD}$ journey. They are like the compass that shows the direction, the wind that blows away the dark clouds, and the harbors for shelters from the storms. With these assistances I am nearing the destination with the joy of knowledge acquisition and personal growth. Therefore, I would like to express my thanks to every person for giving me the support.

First of all, I would like to express my sincere gratitude to my promotor Ivonne Rietjens for her support during my entire $\mathrm{PhD}$. Ivonne, thank you for providing me the opportunity to be a $\mathrm{PhD}$ student at Toxicology. In additional to knowledge, I have also learned a lot from you especially how to work in an efficient and smart way. I really appreciate for your quick and constructive feedback on my papers and thesis in particular at the last stage of my $\mathrm{PhD}$, which gave me the confidence to finish thesis book in time. Also, thanks to your supervision and encouragement, which got me through the period full of confusion and stress to move forward further. I am grateful for your attendance at AstraZeneca event which made me felt to be supported. What I am most grateful for is that your positive attitude towards the difficulties encountered in the research and life influenced my way to deal with challenges and let me know how to enjoy the sense of accomplishment after solving the challenges.

I also would like to express my sincere gratitude to my co-promotors Hans Bouwmeester and Marije Strikwold for their supervision, support and motivation. Hans, thank you for the fruitful discussions in those bi-week meetings for more than three years and they can always make me think from different perspectives. Thank you for being so supportive all the time and giving me the space to explore the topics of my own interest. Without your help, the ibogaine chapter would not be possible. I also appreciated that you always reminded me to balance the time between work and personal life even at the busiest stage of my PhD. Marije, thank you for taking me into the world of in silico modeling which triggered my interests to further explore more exciting things in this field. I enjoyed each meeting with you and the time spent in Leeuwarden for learning modeling. Sometimes we can have long time meeting (more than 2 hours) and long emails to discuss the details about the modeling or manuscript. For this I would like to thank you for being so patients and for the time you dedicated to my $\mathrm{PhD}$ study. I am also grateful for your critical comments and precise attitude towards research which inspired and encouraged me to keep going.

I would like to extend my gratitude to all co-authors of my publications for their contribution. To Jochem Louisse, thank you for asking me if I was interested in doing a PhD during my MSc. That's the beginning of everything. What I learnt from you during my MSc thesis encouraged me to continue with the $\mathrm{PhD}$ study and you let me know it is can be so interesting to do research in toxicology. I really appreciate your help in the preparation for the application of Chinese Scholarship Council Scholarship and your supervision during the first stage of my $\mathrm{PhD}$. Thank you for sharing your ideas and for your support all the time. Laura de Haan, thank you for helping me with the experimental work since my MSc. I learnt from you for almost all the experimental skills related to bioassay used in my $\mathrm{PhD}$ project. Also thank you for bringing a relaxing atmosphere in the lab. Tien Nguyen, thank you for helping me with optimizing the mouse and human cardiotoxicity models, for the useful tips of cell culturing and for sharing your experience of doing postdoc. To Sebas Wesseling, thank you for your knowledge on 
LC-MS analysis, for helping me with setting up and optimizing analytical methods and also for the talks and jokes. I remembered that we discussed how to solve the contamination issue of new LC-MS and finally I got stable results with your help, really thank you for that.

I am highly grateful for Chinese Scholarship Council who approved my application and provided me the financial support for the living expenses in the Netherlands.

I would also like to express my sincere gratitude to the thesis committee members Dr. ir. A. Punt, Prof. dr R.F. Witkamp, Dr. R.H.S. Westerink and Prof. dr. F.G.M. Russel for your time to evaluate this thesis.

I would like to thank the former and current staff members at Toxicology. Bert, thank you for being so friendly and helpful all the time. Your talks and jokes made me feel relax and enjoy the time in lab even with the busy experiments. In particular at the beginning of my PhD I stayed in the lab to collect the peaks from HPLC from morning to evening for almost one month. Thank you for the company and interesting chats! Hans and Wouter, thank you for maintaining the routine work in the labs. Without you our lab could not run smoothly especially during the COVID-19 crisis. Hans, also thank you for the nice organization of bird watching so I had the chance to experience this amazing activity. Hopefully the pandemic will end soon and the yearly TOX bird watching can be continued. Nico and Karsten, thank you both for the constructive comments and inspiring remarks during research in progress presentations. To Lidy, Carla and Gerda, thank you for helping me with all administrative issues. I can always receive the efficient responses. Letty, thank you for the help with administrative matters of the PET course.

To the former and current PhDs at Toxicology, I really appreciate you for the great time and the nice working atmosphere: Mengying, Annelies, Chen, Maartje, Orsolya, Frances, Isaac, Danlei, Jing F, Bohan, Felicia, Edith, Abdul, Amer, Lenny, Marta, Nacho, Qianrui, Katja, Akanksha, Veronique, Yiming, Aafke, Jia, Lu, Ashraf, Gogo, Suparmi, Ixchel, Tessa, Nina, Jingxuan, Phim, Aziza, Diego, Jing J, Menno, Biyao, Shensheng, Liang and Jiaqi. Mengying, thank you for your friendship and your helpful and warmhearted suggestions in both scientific and personal life. I really enjoyed the time spent with you for dinners and trips. Jia and Lu, thanks for all the help with the experimental work and modeling during your $\mathrm{PhD}$ study. Jia, also thanks for all the fruity discussions regarding the modeling even though you are busy with adapting the new stage of your life I can still receive your quick and precise responses to all my questions, really appreciate it. Phim, thank you for being so thoughtful and sharing your experiences with me. Nacho, thanks for warm advice for the $\mathrm{PhD}$ study. It is nice to work with you in the lab and enjoyed the music together. To Qianrui, Ixchel, Danlei, Isaac, Jing F, Jingxuan and Yimin, I am grateful for all the inspiring discussions and suggestions in the labs or in the practical. To Annelies, Katja, Felicia, Isaac and Suparmi, thanks for the nice chats and for always bringing fresh topics. To Bohan and Biyao, thanks for your kindness and warm heart and for your timely help in many ways. Special thanks go to my (former) officemates Diana, Shuo, Mebra, Weijia, Merel and Qiuhui for those happy and great time in room 4037. Diana thank you for being my friend since our MSc time, I still remembered how excited I was when I knew you will also start a PhD at TOX. Thank you for all the thoughtful talks, for the help and for letting me feel being supported during these years. I really miss the time I spent with you and Shuo for nice food and beer. Hopefully one day we can meet again in the Netherlands. To Chen, thank you for being my paranymph. I appreciate all the inspiring talks about both science and life. It is nice to share thoughts with you and I can always get motivated and more 
optimistic. Wish you can finish the $\mathrm{PhD}$ smoothly and get together with your family members soon! Shuo, Lenny, and Shensheng, actually I don't know where to start my thanks. There are too many memorable moments to tell. We share both happiness and worries. Your company and encouragement get me through the difficult time. I feel so lucky to have you as my friends. And I know for sure we will keep in touch!

I would like also to thank my former MSc students for the nice experiences to work with them: Sofia Papadopoulou, Valerie de Boor, Yumeng Dong, Germain Crentsil and Beatrice Rocchi. Special thanks to Yumeng, despite I was supervising your thesis, I was also learning from you. I miss the time we spent together to learn Monte Carlo simulation and appreciate your friendship beyond the research.

I also want to thank my friends in the Netherlands and outside of the Netherlands for their support in many aspects. To Hao, Lei, Liyou, Shuo, Wei, Weijia, Wenhao, Ziwen and Zhengcong, I am grateful for your friendships for these years (since our MSc). We shared the joy of success, the troubles encountered in the $\mathrm{PhD}$, and the worries and hopes about the future. We help each other to keep stronger on the way our $\mathrm{PhD}$ journeys. I cannot wait to meet you all again in China or in the Netherlands. To Qimeng, I really enjoyed the talks that we shared the similar hobbies and the short trips with you. Don't forget our plan to start a podcast! To my dear Zhixin, Lingtian, Rui and Yicheng, thank you for being my close friends for more than 15 years and gave a great support during these four years. You have always been my backing no matter what I encountered.

最重要的是, 我要感谢我的父母为我所做的一切。自大学起我已离家十年, 无论发生什么你们只与 我分享喜悦, 让我没有后顾之忧的专心求学。谢谢你们尊重并支持我来荷兰求学的决定, 即便不舍, 也 给我提供了自由发展的空间。没有你们的关爱, 理解和鼓励, 我无法完成博士学位。

(Mostly importantly, I would like to express my thanks to my parents for everything they have done for me. I have been away from home for more than ten years, no matter what happened you only share good news with me, which allows me to focus on my study without any concerns. After I completed my bachelor's degree, despite you want me to go back to hometown and stay under your care, you still provide me space and let me grow up as I wish. Thank you for being so supportive to my decision to study in the Netherlands. Your love, care, understanding and encouragement are the basis of my $\mathrm{PhD}$.)

Finally, I want to thank myself for never thinking about giving up. The road to finishing a $\mathrm{PhD}$ is not easy but I enjoyed the journey a lot. Again, I am grateful for the things I experienced, the support I got and the people I met. I will take this priceless treasure to my next adventure. 


\section{About the author}

Miaoying was born on $20^{\text {th }}$ September 1991 in Shijiazhuang, Hebei, China. In 2014, Miaoying obtained her BSc in Food Quality and Safety from Yunnan Agricultural University. In August 2014, Miaoying moved to the Netherlands and start her MSc study in Food Safety at Wageningen University and Research. In 2015, she did her thesis at Toxicology department under the supervision of Dr. Jochem Loussie. The thesis project is to investigate the estrogenic potency of

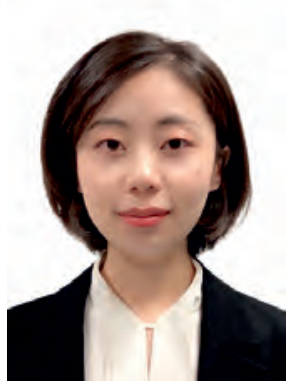
cadmium and aluminum in cell-based and receptor-based in vitro models. After that Miaoying did an internship at Merieux Nutrisciences in the Netherlands. From 2017 Miaoying started her $\mathrm{PhD}$ at Toxicology department in Wageningen University and Research under the supervision of Prof. Dr. Ivonne M. C. M. Rietjens, Dr. Hans Bouwmeester and Dr. Marije Strikwold and with the financial support of from the Chinese Scholarship Council. During her PhD study, she followed the post-graduate education program in Toxicology as part of the training to be registered as European Registered Toxicologist. 


\section{List of publication}

\section{Peer reviewed publications}

Shi, M., Tien, N. T., de Haan, L., Louisse, J., Rietjens, I. M.C.M., Bouwmeester, H. (2020). Evaluation of in vitro models of stem cell-derived cardiomyocytes to screen for potential cardiotoxicity of chemicals. Toxicology in Vitro, 104891.

Shi, M., Bouwmeester, H., Rietjens, I. M.C.M., Strikwold, M. (2020). Integrating in vitro data and physiologically based kinetic modeling-facilitated reverse dosimetry to predict human cardiotoxicity of methadone. Archives of Toxicology, 1-19

Shi, M., Dong, Y., Bouwmeester, H., Rietjens, I. M.C.M., Strikwold, M. In vitro-in silico-based prediction of inter-individual and inter-ethnic variations in the dose-dependent cardiotoxicity of R-and S-methadone in humans. Submitted.

Shi, M., Bouwmeester, H., Rietjens, I. M.C.M. A new approach methodology (NAM) for the prediction of (nor)ibogaine-induced cardiotoxicity in humans. ALTEX (in press).

\section{Conferences abstracts}

Shi, M., Strikwold, M., Rietjens, I.M.C.M., Bouwmeester, H. (2019). Prediction of human cardiotoxicity of methadone by a combined in vitro - physiologically based kinetic (PBK) modellingbased reverse dosimetry approach. Toxicology Letters Special Issue volume 314, S154.

Shi, M., Strikwold, M., Rietjens, I.M.C.M., Bouwmeester, H. (2020). An in vitro physiologically based kinetic (PBK) modelling-based testing strategy to predict human cardiotoxicity of methadone. The Toxicologist Supplement to Toxicological Sciences volume 174, Issue 1. 


\section{Overview of completed training activities}

\section{Discipline specific activities}

Molecular toxicology

Pathobiology

Cell Toxicology

Organ Toxicology

Laboratory of animal science

Epidemiology

Immunotoxicology

Neurotoxicology
PET

2017

PET

2017

PET

2018

PET

2018

PET

2017

PET

2018

PET

2018

PET

\section{Conferences}

$39^{\text {th }}$ Annual meeting of the Dutch Society of Toxicology (NVT), poster, Hilversum, the Netherlands, 2018

$55^{\text {th }}$ congress of the European Societies of Toxicology (EUROTOX), poster, Helsinki, Finland, 2019

$59^{\text {th }}$ Annual (virtual) meeting Society of Toxicology (SOT), poster, California and online, US, 2020

$60^{\text {th }}$ Annual (virtual) meeting Society of Toxicology (SOT), poster, online, 2021

\section{General courses}

VLAG PhD week

Project and time management

Introduction to $\mathrm{R}$

Applied statistics

Philosophy and ethics of food science and technology
WUR

WUR

WUR

WUR

WUR
2017

2017

2019

2019

2020

\section{Other activities}

Preparation of research proposal

TOX-WUR

2017

PhD trip to Japan

TOX-WUR

2018

Scientific presentation at Division of Toxicology

TOX-WUR

Environmental toxicology

WUR

2021

General toxicology

WUR

\section{Approved by the graduate school VLAG}


The research described in this thesis was financially supported by China Scholarship Council (No. 201607720029 to MIAOYING SHI), China

Financial support from Wageningen University for printing this thesis is gratefully acknowledged.

Cover design by Miaoying Shi. Created with BioRender.com.

Printed by ProefschriftMaken || proefschriftmaken.nl 


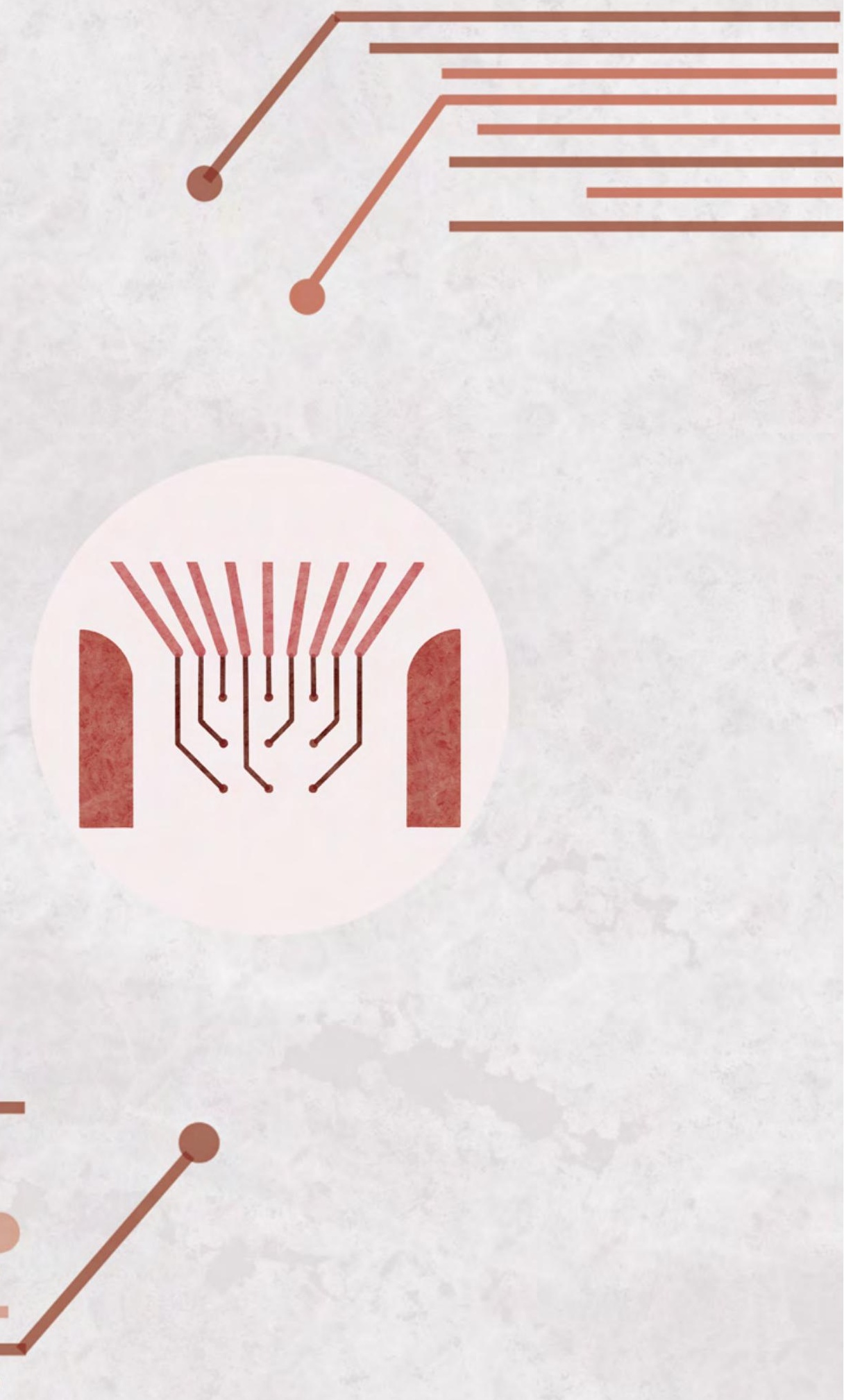